Estudo e desenvolvimento de algoritmos para agrupamento fuzzy de dados em cenários centralizados e distribuídos 



\title{
Estudo e desenvolvimento de algoritmos para agrupamento fuzzy de dados em cenários centralizados e distribuídos
}

\author{
Lucas Vendramin
}

Orientador: Prof. Dr. Ricardo José Gabrielli Barreto Campello

\begin{abstract}
Dissertação apresentada ao Instituto de Ciências Matemáticas e de Computação - ICMC-USP, como parte dos requisitos para obtenção do título de Mestre em Ciências - Ciências de Computação e Matemática Computacional. VERSÃO REVISADA
\end{abstract}


Ficha catalográfica elaborada pela Biblioteca Prof. Achille Bassi e Seção Técnica de Informática, ICMC/USP, com os dados fornecidos pelo(a) autor(a)

\begin{tabular}{|c|c|}
\hline \multirow[t]{3}{*}{ V453e } & $\begin{array}{l}\text { Vendramin, Lucas } \\
\text { Estudo e desenvolvimento de algoritmos para } \\
\text { agrupamento fuzzy de dados em cenários centralizados } \\
\text { e distribuídos / Lucas Vendramin; orientador Ricardo } \\
\text { José Gabrielli Barreto Campello. -- São Carlos, } 2012 \text {. } \\
\quad 138 \text { p. }\end{array}$ \\
\hline & $\begin{array}{l}\text { Dissertação (Mestrado - Programa de Pós-Graduação en } \\
\text { Ciências de Computação e Matemática Computacional) -- } \\
\text { Instituto de Ciências Matemáticas e de Computação, } \\
\text { Universidade de São Paulo, 2012. }\end{array}$ \\
\hline & $\begin{array}{l}\text { 1. Mineração de Dados. 2. Agrupamento de Dados. I. } \\
\text { Campello, Ricardo José Gabrielli Barreto, orient. } \\
\text { II. Título. }\end{array}$ \\
\hline
\end{tabular}




\section{Agradecimentos}

À Deus por me dar, além dos obstáculos, forças para enfrentá-los.

À minha família que sempre me apoiou durante toda minha vida e em minhas decisões. À minha noiva que sempre esteve ao meu lado ajudando a nunca desistir dos desafios. Ao meu orientador por participar de minha formação acadêmica e pelo trabalho sério. Ao professor Eduardo Hruschka pelo coleguismo a grande apoio.

À professora Maria Carolina Monard pelos conselhos e apoio acadêmico. Aos amigos de aulas, trabalho e futebol.

Aos funcionários e professores do ICMC-USP, que sempre foram prestativos e amigos. À Fapesp e ao CNPq pelo auxílio financeiro. 


\section{Resumo}

Agrupamento de dados é um dos problemas centrais na área de mineração de dados, o qual consiste basicamente em particionar os dados em grupos de objetos mais similares (ou relacionados) entre si do que aos objetos dos demais grupos. Entretanto, as abordagens tradicionais pressupõem que cada objeto pertence exclusivamente a um único grupo. Essa hipótese não é realista em várias aplicações práticas, em que grupos de objetos apresentam distribuições estatísticas que possuem algum grau de sobreposição. Algoritmos de agrupamento fuzzy podem lidar naturalmente com problemas dessa natureza. A literatura sobre agrupamento fuzzy de dados é extensa, muitos algoritmos existem atualmente e são mais (ou menos) apropriados para determinados cenários, por exemplo, na procura por grupos que apresentam diferentes formatos ou ao operar sobre dados descritos por conjuntos de atributos de tipos diferentes. Adicionalmente, existem cenários em que os dados podem estar distribuídos em diferentes locais (sítios de dados). Nesses cenários o objetivo de um algoritmo de agrupamento de dados consiste em encontrar uma estrutura que represente os dados existentes nos diferentes sítios sem a necessidade de transmissão e armazenamento/processamento centralizado desses dados. Tais algoritmos são denominados algoritmos de agrupamento distribuído de dados. O presente trabalho visa o estudo e aperfeiçoamento de algoritmos de agrupamento fuzzy centralizados e distribuídos existentes na literatura, buscando identificar as principais características, vantagens, desvantagens e cenários mais apropriados para a aplicação de cada um deles, incluindo análises de complexidade de tempo, espaço e de comunicação para os algoritmos distribuídos. 


\section{Abstract}

Data clustering is a fundamental conceptual problem in data mining, in which one aims at determining a finite set of categories to describe a data set according to similarities among its objects. Traditional algorithms assume that each object belongs exclusively to a single cluster. This may be not realistic in many applications, in which groups of objects present statistical distributions with some overlap. Fuzzy clustering algorithms can naturally deal with these problems. The literature on fuzzy clustering is extensive, several fuzzy clustering algorithms with different characteristics and for different purposes have been proposed and investigated and are more (or less) suitable for specific scenarios, e.g., finding clusters with different shapes or working with data sets described by different types of attributes. Additionally, there are scenarios in which the data are (or can be) distributed among different sites. In these scenarios, the goal of a clustering algorithm consists in finding a structure that describes the distributed data without the need of data and processing centralization. Such algorithms are known as distributed clustering algorithms. The present document aims at the study and improvement of centralized and distributed fuzzy clustering algorithms, identifying the main characteristics, advantages, disadvantages and appropriate scenarios for each application, including complexity analysis of time, space and communication for the distributed algorithms. 
Esta dissertação foi preparada com o formatador de textos LTEX. O estilo utilizado no documento foi desenvolvido por Ronaldo Cristiano Prati. A bibliografia foi gerada automaticamente pelo BibTEX, utilizando o estilo Chicago com modificações para o português. Algumas palavras utilizadas neste trabalho não foram traduzidas da língua inglesa para a portuguesa por serem amplamente conhecidas e difundidas na comunidade acadêmica. 


\section{Sumário}

Lista de Figuras . . . . . . . . . . . . . . . . . . . . . . . . xvii

Lista de Tabelas . . . . . . . . . . . . . . . . . . . . . . xix

Lista de Algoritmos . . . . . . . . . . . . . . . . . . . xxii

1 Introdução 1

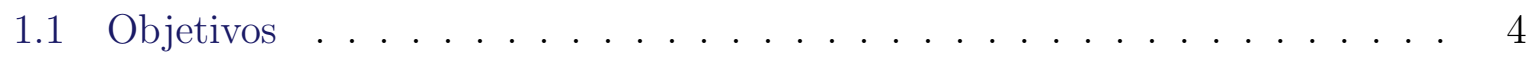

1.2 Organização do Trabalho . . . . . . . . . . . . . . . . . . . . 4

2 Agrupamento Fuzzy de Dados $\quad 7$

2.1 Definição do Problema . . . . . . . . . . . . . . . . 7

2.2 Algoritmos de Agrupamento Fuzzy de Dados . . . . . . . . . . . . . . . . . 10

2.2 .1 FCM: Fuzzy c-Means . . . . . . . . . . . . . . . . . . . . 11

2.2.2 Algoritmos que Buscam por Grupos Hiperelípticos . . . . . . . . . . 14

2.2.2.1 GK: Gustafson-Kessel . . . . . . . . . . . . . . . 14

2.2.2.2 GG: Gath and Geva . . . . . . . . . . . . . . 15

2.2.3 Algoritmos que Utilizam Protótipos Não Pontuais . . . . . . . . . . 17

2.2.3.1 FCV: Fuzzy c-Varieties . . . . . . . . . . . . . . 17

2.2.3.2 FCE: Fuzzy c-Elliptotypes . . . . . . . . . . . . . . 18

2.2.3.3 EFCM: Extended Fuzzy c-Means . . . . . . . . . . . . 20

2.2.3.4 PLFC: Prototype-less Fuzzy Clustering . . . . . . . . . . 22

2.2.4 Algoritmos que Buscam por Contornos . . . . . . . . . . . . . . 23

2.2.4.1 FCS: Fuzzy c-Shells . . . . . . . . . . . . . . . 24

2.2.4.2 FCSS: Fuzzy c-Spherical Shells . . . . . . . . . . . 25

2.2 .5 Algoritmos Possibilísticos . . . . . . . . . . . . . . . . . 27

2.2.5.1 PCM: Possibilistic c-Means . . . . . . . . . . . . . 27

2.2.5.2 PGK: Possibilistic Gustafson-Kessel ....... . 29

2.2.5.3 FPCM: Fuzzy-Possibilistic c-Means . . . . . . . . . . . . 30

2.2.5.4 PFCM: Possibilistic-Fuzzy c-Means . . . . . . . . . . . . . 32

2.2.6 Algoritmos Relacionais . . . . . . . . . . . . . . . . . . . . 34

2.2.6.1 FCMdd: Fuzzy c-Medoids . . . . . . . . . . . . . . 35 
2.2.6.2 FCTMdd: Fuzzy c-Trimmed Medoids . . . . . . . . . . . 36

2.2.6.3 RFCM: Relational Fuzzy c-Means . . . . . . . . . . . . . . 38

2.2.6.4 NERFCM: Non-Euclidean Relational Fuzzy c-Means . . . 39

2.2.7 Algoritmos que Lidam com Valores Ausentes . . . . . . . . . . . . . 41

2.2.7.1 WDSFCM: Whole Data Strategy Fuzzy c-Means . . . . . 42

2.2.7.2 PDSFCM: Partial Distance Strategy Fuzzy c-Means . . . 43

2.2.7.3 OCSFCM: Optimal Completion Strategy Fuzzy c-Means . 44

2.2.7.4 NPSFCM: Nearest Prototype Strategy Fuzzy c-Means . . 45

2.2.8 Algoritmos que Buscam Diminuir o Tempo de Processamento . . . 46

2.2.8.1 brFCM ................... . . 46

2.2.8.2 mrFCM: Multistage Random Sampling Fuzzy c-Means . . 49

2.2.8.3 linear-FCM . . . . . . . . . . . . . . . 51

2.2.9 Algoritmos que Lidam com Atributos Categóricos . . . . . . . . . . 51

2.2.9.1 FSCM: Fuzzy Symbolic c-Means . . . . . . . . . . . . . 52

2.2.10 Análise de Complexidade - Resumo . . . . . . . . . . . . . . . . 54

2.3 Outros Algoritmos . . . . . . . . . . . . . . . . . . 54

2.4 Escolha do Algoritmo de Agrupamento . . . . . . . . . . . . . . . . . 57

2.5 Validação de Agrupamento Fuzzy . . . . . . . . . . . . . . . . . . 58

2.5.1 Índices Baseados Apenas na Matriz de Partição . . . . . . . . . . . 59

2.5.1.1 PC: Partition Coefficient . . . . . . . . . . . . 59

2.5.1.2 PE: Partition Entropy . . . . . . . . . . . . . . 59

2.5.1.3 MPC: Modified Partition Coefficient . . . . . . . . . 60

2.5.2 Índices Baseados na Matriz de Dados . . . . . . . . . . . . . . 61

2.5.2.1 XB: Xie-Beni . . . . . . . . . . . . . . 61

2.5.2.2 FSS: Fuzzy Simplified Silhouette . . . . . . . . . . . . 61

2.5.2.3 K: Kwon . . . . . . . . . . . . . . . . . . 62

2.5.2.4 TSS: Tang-Sun-Sun . . . . . . . . . . . . . 63

2.5.2.5 FS: Fukuyama-Sugeno . . . . . . . . . . . . . . 63

2.5.2.6 FHV: Fuzzy Hypervolume . . . . . . . . . . . . . . . . 64

2.5.2.7 APD: Average Partition Density . . . . . . . . . . 65

2.5.2.8 PD: Partition Density . . . . . . . . . . . . 65

2.5.2.9 SCG ....................... 66

2.5.2.10 PBMF . . . . . . . . . . . . . . 67

2.5.3 Análise de Complexidade - Resumo . . . . . . . . . . . . . 67

2.6 Estimação do Número de Grupos . . . . . . . . . . . . . . . . . . . 67

3 Agrupamento de Dados Distribuído $\quad 71$

3.1 Generalização dos Algoritmos e Índices Estudados . . . . . . . . . . . . . . 74

3.1.1 DFCM: Distributed Fuzzy c-Means . . . . . . . . . . . . . . 75

3.1.2 Framework para Dados Distribuídos . . . . . . . . . . . . . 77

3.1.3 Outros Algoritmos de Agrupamento Fuzzy Distribuídos . . . . . . . 79 
3.1.4 Análise de Complexidade - Comunicação . . . . . . . . . . . . . . . 81

3.1.5 Validação Distribuída de Agrupamento . . . . . . . . . . . . . . . . 81

3.1.5.1 DPC: Distributed Partition Coefficient . . . . . . . . . . 82

3.1.5.2 DPE: Distributed Partition Entropy . . . . . . . . . . 83

3.1.5.3 DMPC: Distributed Modified Partition Coefficient . . . . 84

3.1.5.4 DXB: Distributed Xie-Beni . . . . . . . . . . . . 84

3.1.5.5 DFSS: Distributed Fuzzy Simplified Silhouette. . . . . . . 84

3.1.5.6 DK: Distributed Kwon . . . . . . . . . . . . . 85

3.1.5.7 DTSS: Distributed Tang-Sun-Sun . . . . . . . . . . 86

3.1.5.8 DFS: Distributed Fukuyama-Sugeno . . . . . . . . . . 86

3.1.5.9 DFHV: Distributed Fuzzy Hypervolume . . . . . . . . . . 87

3.1.5.10 DAPD: Distributed Average Partition Density . . . . . . . 87

3.1.5.11 DPD: Distributed Partition Density . . . . . . . . . 87

3.1.5.12 DSCG: Distributed SCG . . . . . . . . . . . . . 87

3.1.5.13 DPBMF: Distributed PBMF . . . . . . . . . 88

3.1.5.14 Análise de Complexidade - Comunicação . . . . . . . . . . 88

3.1.6 Estimação do Número de Grupos . . . . . . . . . . . . . . . . . . 90

3.1.7 Tabela de Cálculos para Cenários Distribuídos . . . . . . . . . . . . 91

3.2 Agrupamento em Consenso . . . . . . . . . . . . . . . . . . . . . 92

3.2.1 ACO-H: Agrupamento em Consenso Original - Caso Horizontal . . 93

3.2.2 ACM-H: Agrupamento em Consenso Modificado - Caso Horizontal 95

3.2.3 Estimação do Número de Grupos . . . . . . . . . . . . . . . . . . 97

3.2.4 Eliminação de Grupos Não Representativos . . . . . . . . . . . . . 98

3.3 Dados Distribuídos Verticalmente . . . . . . . . . . . . . . . . . . . 99

3.3.1 ACO-V: Agrupamento em Consenso Original - Caso Vertical . . . 100

3.3.2 ACM-V: Agrupamento em Consenso Modificado - Caso Vertical . 101

3.3.3 Estimação do Número de Grupos . . . . . . . . . . . . . . . . . . . 103

\section{Resultados}

4.1 Experimentos com Algoritmos Paralelos/Distribuídos . . . . . . . . . . . 105

4.2 Experimentos com Algoritmos de Consenso . . . . . . . . . . . . . . . . . 108

4.3 Caso Horizontal - I . . . . . . . . . . . . . . . . . . . . . . . . . . . . . 111

4.4 Caso Horizontal - II . . . . . . . . . . . . . . . . . . . . . . . . . . . . . . . 114

4.5 Caso Vertical - I . . . . . . . . . . . . . . . . . . . . . . . . . . . . . 118

4.6 Caso Vertical - II . . . . . . . . . . . . . . . . . . . . . . . . . . . . . 120

5 Conclusões e Trabalhos Futuros $\quad 123$

5.1 Conclusões . . . . . . . . . . . . . . . . . . . . . . . . 123

5.2 Contribuições . . . . . . . . . . . . . . . . . . . . . . . . . . . . 124

5.3 Publicações Geradas . . . . . . . . . . . . . . . . . . 125

5.4 Trabalhos Futuros . . . . . . . . . . . . . . . . . . 125 
A Índices Externos 


\section{Lista de Figuras}

2.1 Exemplo de grau de pertinência de dois objetos (representados por pontos) a dois grupos (cujos protótipos estão representados por estrelas). . . . . . . 27

3.1 Agrupamento de dados com e sem a centralização dos dados. . . . . . . . . 73

3.2 Dados (a) distribuídos horizontalmente (b) . . . . . . . . . . . . . 73

3.3 Dados (a) distribuídos verticalmente (b) . . . . . . . . . . . . . 74

3.4 Agrupamento em Consenso - Caso Horizontal. . . . . . . . . . . . . . . . 94

4.1 Speedup obtido pelos algoritmos de agrupamento distribuídos executados em uma base de dados com $2^{10}$ objetos após 10 e 100 iterações. . . . . . . . 106

4.2 Speedup obtido pelos algoritmos de agrupamento distribuídos executados em uma base de dados com $2^{13}$ objetos após 10 e 100 iterações. . . . . . . . . 107

4.3 Speedup obtido pelos algoritmos de agrupamento distribuídos executados em uma base de dados com $2^{17}$ objetos após 10 e 100 iterações. . . . . . . . . 107

4.4 Base de dados de exemplo e o resultado do algoritmo FCM. . . . . . . . . 109

4.5 Resultado do algoritmo FCM aplicado individualmente em cada subespaço com $k=2$ grupos — centros são representados por "estrelas" — o maior valor de pertinência de cada objeto é representado pela intensidade de preto.109

4.6 Resultado do algoritmo ACO-V executado para as soluções obtidas individualmente em cada subespaço ilustradas na Figura 4.5 - centros são representados por "estrelas" — o maior valor de pertinência de cada objeto é representado pela intensidade de preto . . . . . . . . . . . . . . 110

4.7 Resultado do algoritmo ACM-V executado para as soluções obtidas individualmente em cada subespaço ilustradas na Figura 4.5 - centros são representados por "estrelas" - o maior valor de pertinência de cada objeto é representado pela intensidade de preto . . . . . . . . . . . . . . 110

4.8 Dois sítios de dados. Cada sítio de dados possui $N=40$ objetos descritos pelos mesmos $n=2$ atributos. . . . . . . . . . . . . . . . . 111 
4.9 Melhor partição fuzzy obtida pelo procedimento DOMR-DFCM para os sítios de dados ilustrados na Figura 4.8. Os sítios de dados estão ilustrados juntos (centralizados) — os centros são representados por "estrelas" — o maior valor de pertinência de cada objeto é representado pela intensidade de preto. . . . . . . . . . . . . . . . . . . . . . 112

4.10 Melhor partição fuzzy obtida pelo procedimento DOMR-DFCM para os sítios de dados da Figura 4.8. Cada sítio de dados é ilustrado separadamente — os centros são representados por "estrelas" - o maior valor de pertinência de cada objeto é representado pela intensidade de preto. . . . . 112

4.11 Melhor partição obtida pelo procedimento DOMR-FCM quando aplicado a cada sítio de dados individualmente.

4.12 Melhor resultado obtido pelo processo de consenso: (a) consenso aplicado considerando o sítio de dados 1 como local; (b) consenso aplicado considerando o sítio de dados 2 como local. . . . . . . . . . . . . . . . . . . . . . 113

4.13 Três sítios de dados. Cada um possui $N=50$ objetos descritos por $n=2$ atributos. . . . . . . . . . . . . . . . . . 115

4.14 Melhor partição fuzzy obtida pelo procedimento OMR-DFCM para os sítios de dados da Figura 4.13. . . . . . . . . . . . . . . . . . . . 116

4.15 Melhor partição obtida pelo procedimento OMR-FCM em cada sítio de dados individualmente. . . . . . . . . . . . . . . . . . 116

4.16 Resultado obtido pelo processo de consenso: (a) consenso aplicado considerando o sítio de dados 1 como local; (b) consenso aplicado considerando o sítio de dados 2 como local; (c) consenso aplicado considerando o sítio de dados 3 como local. . . . . . . . . . . . . . . . . . . . . . 117

4.17 Base de dados com quatro grupos naturais descritos por $n=2$ atributos. . 118

4.18 Solução encontrada pelo procedimento OMR-FCM na base de dados da Figura 4.17 . . . . . . . . . . . . . . . . . . . . . . . . 119

4.19 Solução encontrada pelo procedimento OMR-FCM no primeiro subespaço. 119

4.20 Solução encontrada pelo procedimento OMR-FCM no segundo subespaço. . 119

4.21 Resultado obtido ao aplicar o processo de consenso em cada subespaço da base de dados da Figura 4.17. Quatro grupos foram encontrado (diamantes, círculos, triângulos e quadrados) - centros são representados por "estrelas". 120

4.22 Dois exemplos de imagens $422 \ldots$. . . . . . . . . . . . . . . . 121

4.23 Dois exemplos de imagens 656. . . . . . . . . . . . . . . . 121

4.24 Dois exemplos de imagens $792 \ldots$. . . . . . . . . . . . . . . 121

4.25 Dois exemplos de imagens $915 \ldots$. . . . . . . . . . . . . . . . 121

4.26 Dois exemplos de imagens $959 \ldots \ldots$. . . . . . . . . . . . . . . 122

A.1 Exemplo de uma solução contendo 3 grupos (brancos, cinzas e pretos) em comparação com outra solução contendo 2 grupos (círculos e quadrados). 138 


\section{Lista de Tabelas}

2.1 Exemplo de matriz de dados com quatro objetos descritos por três atributos. 8

2.2 Exemplo de matriz de partição rígida de quatro objetos e dois grupos. . . . 9

2.3 Exemplo de matriz de partição fuzzy de quatro objetos e dois grupos. . . . 9

2.4 Exemplo de matriz de partição fuzzy probabilística de quatro objetos e dois grupos. . . . . . . . . . . . . . . . . . . 10

2.5 Matriz de dados com quatro objetos descritos por três atributos. . . . . . . 35

2.6 Matriz relacional dos dados contendo as distâncias Euclideanas quadradas entre os objetos ilustrados na Tabela 2.5 . . . . . . . . . . . . . . 35

2.7 Matriz de dados com cinco objetos descritos por três atributos. . . . . . . . 41

2.8 Matriz de dados com dois objetos descritos por três atributos. . . . . . . . 42

2.9 Quatro objetos representados por apenas um atributo numérico. Representação binária e quantizada também estão ilustradas na tabela. . . . . . . 48

2.10 Complexidades de tempo e espaço para os algoritmos de agrupamento fuzzy: $t$ é o número de iterações, $k$ é o número de grupos, $n$ é o número de atributos, $N$ é o número de objetos, $t_{N}$ é o número de iterações do método de Newton e $r$ é a dimensão das variedades. . . . . . . . . . . . . . . 55

2.11 Complexidades de tempo e espaço para os algoritmos de agrupamento fuzzy: $t$ é o número de iterações, $k$ é o número de grupos, $n$ é o número de atributos, $N$ é o número de objetos, $s$ é o número de objetos para o cálculo do medóide, $N^{0}$ é o número de objetos distintos da base de dados, $\Delta_{\%}$ é a porcentagem das amostras, $r$ é o número de amostras e $n_{p}$ é a quantidade de valores diferentes do $p$-ésimo atributo. . . . . . . . . . . . . . 56

2.12 Complexidade de tempo e espaço para os índices de validade de agrupamento fuzzy: $k$ é o número de grupos, $n$ é o número de atributos e $N$ é o número de objetos. . . . . . . . . . . . . . . . . .

3.1 Complexidade de comunicação para os algoritmos de agrupamento fuzzy distribuídos: $P$ é o número de sítios de dados, $t$ é o número de iterações, $k$ é o número de grupos e $n$ é o número de atributos. . . . . . . . . . . . . 
3.2 Complexidade de comunicação para os algoritmos de agrupamento fuzzy distribuídos: $P$ é o número de sítios de dados, $t$ é o número de iterações, $k$ é o número de grupos, $n$ é o número de atributos, $r$, no algoritmo DmrFCM, é o número de amostras e $n_{p}$, no algoritmo DFSCM, é a quantidade de valores diferentes do $p$-ésimo atributo. . . . . . . . . . . . . . 83

3.3 Complexidade de Comunicação dos índices distribuídos: $P$ é o número de sítio de dados, $k$ é o número de grupos e $n$ é o número de atributos. . . . . 89

3.4 Cálculo dos elementos usados pelos algoritmos e índices distribuídos utilizando (3.8): $P$ é o número de sítios de dados, $k$ é o número de grupos, $m$ e $\gamma$ são coeficientes de fuzzificação, $N_{i i}$ é o número de objetos do $i i$-ésimo sítio de dados, $u[i i]_{i j}$ e $p[i i]_{i j}$ são as pertinências probabilísticas e possibilísticas, respectivamente, do $j$-ésimo objeto do $i i$-ésimo sítio de dados ao $i$-ésimo grupo, $I[i i]_{j p}$ é igual a 1 quando o $p$-ésimo atributo do $j$-ésimo objeto do $i i$-ésimo sítio de dados está presente (caso contrário é igual a 0 ) e $w[i i]_{j}$ é a quantidade de objetos (na matriz de dados original do $i$-ésimo sítio de dados) que são representados pelo elemento $\mathbf{x}^{\prime}[i i]_{j} \ldots$. . . . . . . . . . . . 91

3.5 Cálculo dos índices distribuídos de acordo com (3.8) . . . . . . . . . . . . 92

3.6 Matriz de partição de 7 objetos e 4 grupos. . . . . . . . . . . . . . . . 99

3.7 Matriz de partição — grupo não representativo foi eliminado. . . . . . . . . 99

4.1 Quantidade de transferência (em Kbytes) entre os sítios de dados dos algoritmos usando dois, três e quatro processadores após 100 iterações. . . . . . 108

4.2 Número de grupos, valor de Jaccard e Rand-Ajustado obtidos pelo procedimento OMR-FCMdd em cada subespaço individualmente, pelo procedimento OMR-FCMdd em $R^{+}$e pelo consenso (OMR-ACM-V). . . . . . . . 122 


\section{Lista de Algoritmos}

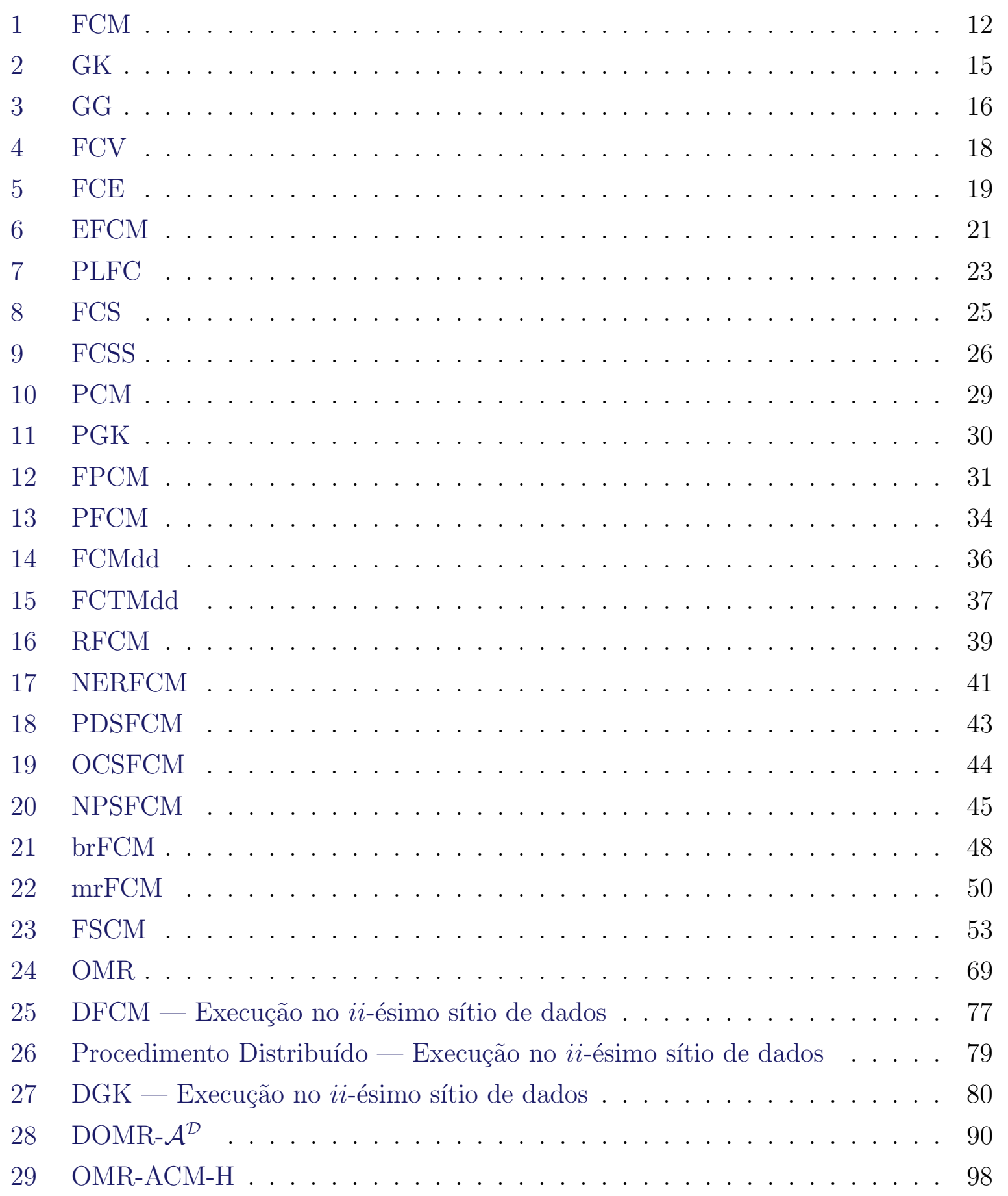




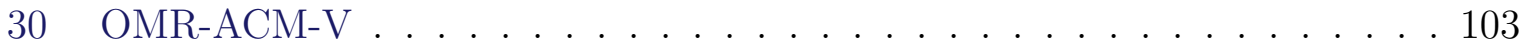




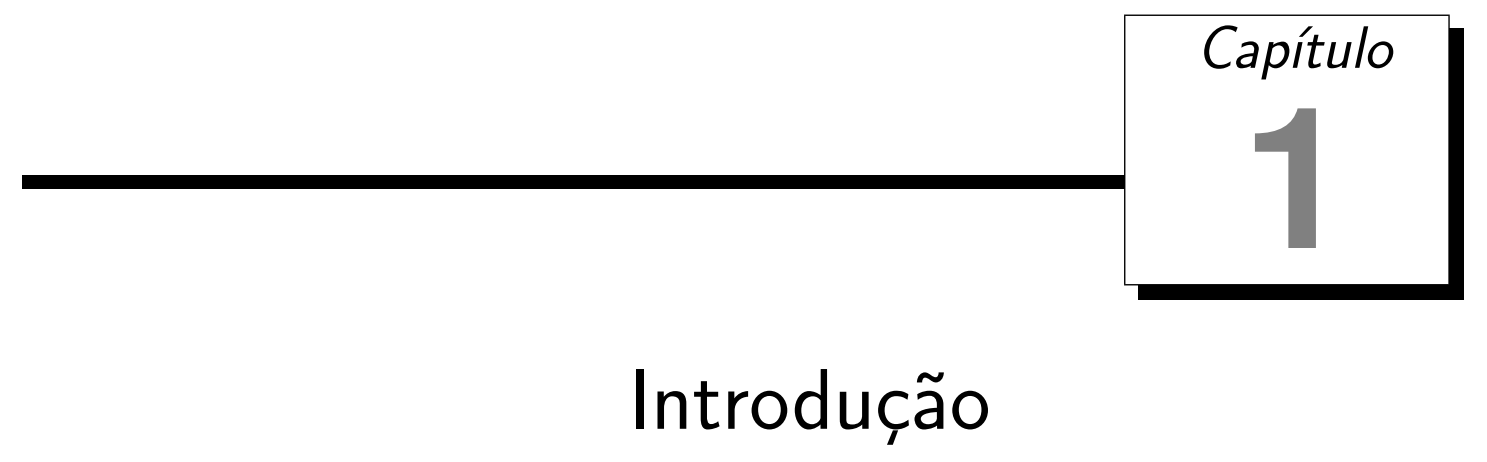

Grandes avanços em tecnologias para coleção e armazenamento de dados têm feito com que enormes quantidades de dados sejam armazenadas nas organizações. A extração de informação útil em tais conjuntos de dados é uma tarefa difícil e desafiadora. Nesse contexto, Mineração de Dados consiste na tecnologia para processar e extrair informações úteis que estão armazenadas em grandes bases de dados (Tan et al., 2005). Formalmente, mineração de dados se constitui de alguns passos de um processo mais amplo, denominado Descoberta de Conhecimento, que consiste de vários processos para converter dados "brutos" em informações úteis (Fayyad et al., 1996; Tan et al., 2005).

Dentre várias tarefas desempenhadas em mineração de dados, Agrupamento de Dados é um dos problemas centrais, o qual consiste em determinar um conjunto de categorias para descrever uma coleção de objetos de acordo com as suas similaridades ou interrelacionamentos (Kaufman \& Rousseeuw, 1990; Everitt et al., 2001). A solução para esse problema consiste frequentemente no objetivo final de mineração de dados, apresentando uma ampla aplicabilidade em diversas áreas, e.g (Everitt et al., 2001; Tan et al., 2005):

- Biologia: Agrupamento de dados é muitas vezes usado para auxiliar na categorização automática de espécies biológicas. Muitas vezes usado também para encontrar grupos de genes que apresentam funções similares.

- Recuperação de Informação: A World Wide Web consiste de bilhões de páginas. Dessa forma, uma simples consulta em um mecanismo de busca pode retornar milhares de páginas. Agrupamento de dados pode ser usado para agrupar tais resultados em um pequeno número de páginas que apresentam um aspecto particular da busca.

- Clima: Para entender o clima na Terra é importante encontrar padrões na atmosfera 
e no oceano. Assim, agrupamento de dados pode ser usado para encontrar padrões na pressão atmosférica e áreas do oceano que apresentam impactos significativos no clima.

- Medicina: Uma doença frequentemente apresenta diversas variações. Agrupamento de dados pode ser adotado para identificar tais subcategorias. Por exemplo, pode ser usado para identificar diferentes tipos de depressão.

- Atividade Comercial: Agrupamento de dados pode ser usado para segmentar consumidores que apresentem características similares. Tais grupos podem ser analisados separadamente e diferentes estratégias podem ser adotadas para cada grupo, e.g., antes de aplicar uma estratégia de marketing.

Adicionalmente, a solução de um problema de agrupamento também pode ajudar a solucionar outros problemas relacionados, tais como classificação de padrões, extração de regras em bases de dados, sumarização de documentos e compressão de dados (Wang \& Fu, 2005; Tan et al., 2005).

A complexidade do problema de agrupamento de dados advém em boa parte da sua natureza não supervisionada, em que não se dispõe objetivamente de um resultado final desejado. Em outras palavras, em contraste aos problemas supervisionados, como classificação de padrões, não se dispõe, em agrupamento de dados, de uma meta concreta a ser alcançada. Na verdade, a dificuldade do problema começa pela própria definição do que se entende por grupo (cluster), conceito com elevado grau de subjetividade. É importante citar também que, na maioria dos casos, existe uma variedade de categorizações alternativas para um mesmo conjunto de objetos, ou seja, os objetos podem ser agrupados de maneiras diferentes dependendo da perspectiva. Por exemplo, um sistema bancário pode estar interessado em encontrar grupos nos quais os objetos (clientes) do mesmo grupo apresentem informações econômicas similares, i.e., levando em conta informações como: renda familiar, quantidade de pessoas na família e quantidade de bens adquiridos, por exemplo. Note que o mesmo sistema bancário também pode estar interessado em encontrar grupos nos quais os objetos do mesmo grupo apresentem informações geográficas similares, i.e., levando em conta informações como: endereço de residência e endereço de contato, por exemplo.

Os algoritmos tradicionais assumem que os objetos pertencem exclusivamente a um único grupo. Contudo, algumas aplicações apresentam grupos de objetos com distribuições estatísticas que, embora distintas e possivelmente bem definidas, possuem algum grau de sobreposição. Em outras palavras, os dados muitas vezes compreendem categorias que se sobrepõem umas às outras em algum grau. Nessas circunstâncias, recomenda-se a utilização de algoritmos de agrupamento com capacidade de lidar com tal característica. Algoritmos de agrupamento fuzzy de dados, que constituem o foco de interesse do presente trabalho, podem lidar naturalmente com esse tipo de problema, pois buscam encontrar grupos difusos (fuzzy clusters — em inglês) (Hoppner et al., 1999). 
Vários algoritmos de agrupamento de dados com diferentes características e para diferentes finalidades vêm sendo propostos e investigados durante as últimas décadas. Especificamente, na área de agrupamento fuzzy de dados, existe um impressionante número de diferentes algoritmos baseados em modificações e/ou extensões da formulação de um dos algoritmos mais conhecidos e estudados, denominado Fuzzy c-Means (FCM - em inglês) (Dunn, 1973; Bezdek, 1981). Por exemplo, grupos hiper-esféricos são induzidos quando a norma Euclideana utilizada no algoritmo original é adotada como medida de distância, porém existem vários algoritmos que se baseiam no FCM original e procuram por grupos com formas diferentes (Bezdek \& Dunn, 1975; Gustafson \& Kessel, 1978; Gath \& Geva, 1989). Neste trabalho, essas e diversas outras variantes são estudadas de maneira criteriosa, apresentando cenários promissores de aplicação e desempenho computacional de cada uma delas.

Ambientes distribuídos tais como a internet, intranets e ambiente de processamento paralelo vêm sendo adotados em diversas áreas de pesquisa e em ambientes práticos de aplicação (Attiya \& Welch, 2004; Cristea et al., 2010), incluindo as áreas de descoberta de conhecimento, aprendizado de máquina, mineração de dados e agrupamento de dados (Park et al., 2001; Park \& Kargupta, 2002; Tsoumakas et al., 2004; Datta et al., 2006). Tal tendência deu origem a áreas de estudo denominadas Descoberta de Conhecimento Distribuído (Distributed Knowledge Discovery in Databases — em inglês) (Zaki \& Pan, 2002; Congiusta et al., 2007), Mineração de Dados Paralela e Distribuída (Parallel and Distributed Data Mining — em inglês) (Johnson \& Kargupta, 2000; Kargupta et al., 2001; Park \& Kargupta, 2002; Zaki \& Pan, 2002; Klusch et al., 2003; Zhang et al., 2004; da Silva et al., 2005; Merugu \& Ghosh, 2005; da Silva \& Klusch, 2007; Congiusta et al., 2007) e Agrupamento de Dados Paralelo e Distribuído (Parallel and Distributed Data Clustering — em inglês) (da Silva \& Klusch, 2006; Merugu \& Ghosh, 2005; Klusch et al., 2003).

Vários algoritmos de agrupamento de dados foram propostos e investigados na literatura para encontrar estruturas em ambientes paralelos e distribuídos (Olson, 1995; Dhillon \& Modha, 2000; Tian et al., 2005; Du \& Lin, 2005; Garg et al., 2006; Hammouda \& Kamel, 2009; Datta et al., 2009). Mais especificamente, alguns foram desenvolvidos como generalizações de versões centralizadas de um algoritmo específico (Olson, 1995; Dhillon \& Modha, 2000; Forman \& Zhang, 2000; Garg et al., 2006), sendo capazes de produzir os mesmos resultados finais que seriam obtidos pelos respectivos algoritmos originais se estes pudessem ser aplicados aos dados de forma centralizada. Embora existam muitos algoritmos capazes de lidar com dados paralelos e distribuídos, poucos foram desenvolvidos para agrupamento fuzzy de dados como generalizações de versões centralizadas de determinado algoritmo (Kwok et al., 2002; Rahimi et al., 2004; Modenesi et al., 2007). Em outras palavras, poucos algoritmos de agrupamento fuzzy de dados foram generalizados para trabalhar com dados paralelos e distribuídos de forma a produzir os mesmos resultados finais que a versão centralizada de tal algoritmo obteria com os dados centralizados. Adicionalmente, muitos algoritmos de agrupamento de dados foram propostos e 
investigados na literatura para encontrar estruturas em ambientes paralelos e distribuídos seguindo uma abordagem diferente. Alguns desses algoritmos, denominados algoritmos de agrupamento colaborativos (collaborative clustering — em inglês), são executados em cada sítio de dados e, iterativamente, compartilham informações com os demais sítios com o objetivo de encontrar uma estrutura local usando informações dos demais sítios (Pedrycz, 2002, 2007; Pedrycz \& Rai, 2008; Coletta et al., 2012). Uma abordagem relacionada executa o algoritmo de agrupamento (centralizado) em cada sítio de dados e, após obter uma partição final, usa alguma espécie de combinação (ensemble - em inglês) com o objetivo de melhorar os resultados obtidos em um sítio específico com informações fornecidas pelos demais sítios de dados (Dimitriadou et al., 2002; Strehl \& Ghosh, 2003; Agogino \& Tumer, 2006; Tumer \& Agogino, 2008). Essa última abordagem é algumas vezes denominada agrupamento em consenso (consensus clustering — em inglês) (Ghosh et al., 2002; Pedrycz \& Hirota, 2008; Vendramin et al., 2011).

Tanto os algoritmos de agrupamento fuzzy centralizados, como os algoritmos de agrupamento fuzzy paralelos/distribuídos fazem parte do escopo do presente trabalho e são discutidos nos próximos capítulos.

\subsection{Objetivos}

Este trabalho de mestrado tem como objetivo estudar algoritmos de agrupamento fuzzy de dados, tanto centralizados como distribuídos, destacando suas características, limitações e cenários mais apropriados para aplicação.

Também se tem como objetivo generalizar vários algoritmos de agrupamento fuzzy de dados para trabalhar no contexto paralelo/distribuído dos dados, além de também aperfeiçoar alguns dos algoritmos investigados, como, por exemplo, o algoritmo para agrupamento distribuído proposto por Loia et al. (2007); Pedrycz \& Hirota (2008).

Para cada algoritmo foi analisado a complexidade computacional em relação ao tempo de execução, espaço necessário e, nos cenários distribuídos, comunicação dos dados. Pretende-se também obter um mecanismo de detecção automática do número de grupos. No caso dos algoritmos distribuídos, pretende-se desenvolver métodos para avaliar a qualidade das soluções obtidas quando não se dispõe de acesso simultâneo a todos os dados.

\subsection{Organização do Trabalho}

O presente trabalho está organizado da seguinte maneira:

Capítulo 2: É apresentada uma revisão sobre agrupamento fuzzy de dados. São descritos os conceitos, as aplicações e a definição do problema. Além disso, são abordados algoritmos de agrupamento amplamente utilizados e importantes para este projeto. 
Por fim, são apresentados critérios numéricos para a validação de soluções encontradas por tais algoritmos de agrupamento.

Capítulo 3: São abordados os conceitos e métodos de agrupamento de dados distribuídos. São apresentados alguns algoritmos de agrupamento de dados em cenários distribuídos e paralelos. Além disso, é apresentado um framework para generalizar os algoritmos de agrupamento fuzzy de dados para trabalharem no cenário paralelo/distribuído. Também são apresentadas melhorias nos algoritmos distribuídos estudados. Mais especificamente, um método para detectar automaticamente o número de grupos nos algoritmos estudados e uma nova formulação para o agrupamento do tipo consenso são apresentados.

Capítulo 4: São apresentados os resultados experimentais obtidos. Estes experimentos ilustram o desempenho computacional dos algoritmos sendo executados no contexto paralelo e ilustram cenários em que os algoritmos de agrupamento fuzzy em consenso podem ser aplicados com sucesso.

Capítulo 5: São apresentadas as conclusões e perspectivas para trabalhos futuros. 


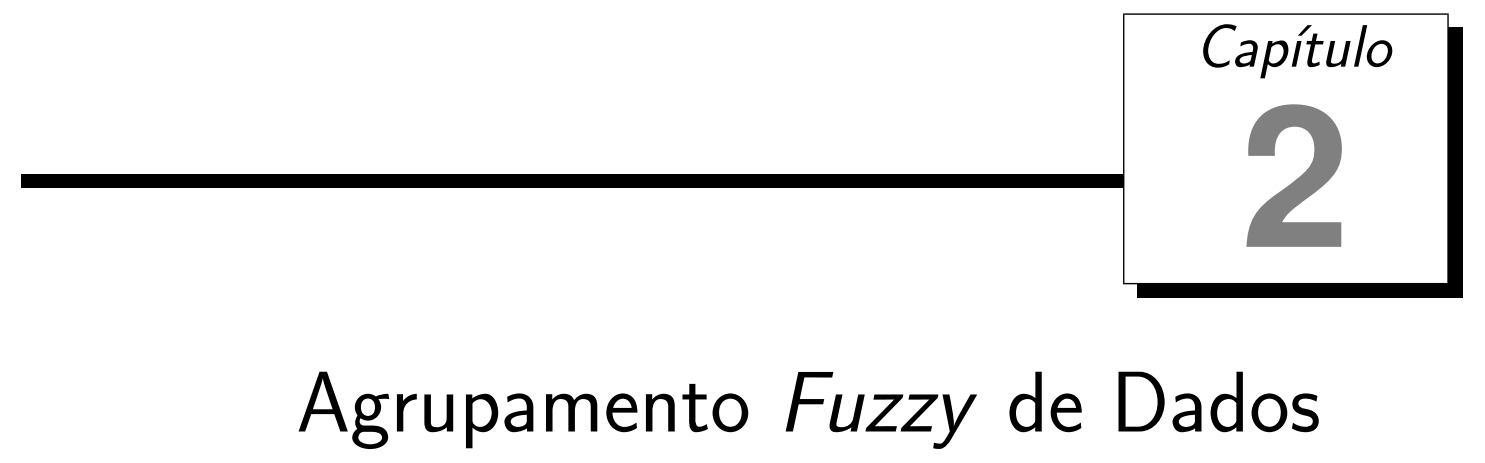

As abordagens tradicionais para agrupamento de dados pressupõem que cada objeto pertence única e exclusivamente a um grupo específico. No entanto, essa hipótese pode não ser realista em algumas aplicações práticas, nas quais grupos de objetos (e.g. indivíduos) apresentam usualmente distribuições estatísticas que, embora distintas e possivelmente bem definidas, possuem algum grau de sobreposição. Em outras palavras, os dados muitas vezes compreendem categorias que se sobrepõem umas às outras em algum grau. Algoritmos de agrupamento fuzzy de dados podem lidar naturalmente com esse tipo de problema, uma vez que buscam encontrar grupos difusos (fuzzy clusters - em inglês), i.e., todo objeto do conjunto de dados pertence a todos os grupos em algum grau quantificado no intervalo [0,1] (Hoppner et al., 1999). Neste capítulo é feito um estudo de alguns dos algoritmos de agrupamento fuzzy de dados mais conhecidos e utilizados na literatura.

\subsection{Definição do Problema}

Agrupamento de Dados é uma tarefa cujo objetivo consiste em determinar um conjunto finito de categorias que descrevem os dados de acordo com as similaridades entre seus objetos. Abstraindo variantes conceituais menores, pode-se dizer que existem três tipos fundamentais de agrupamento de dados (Jain \& Dubes, 1988): com sobreposição, particional e hierárquico. Os últimos dois estão relacionados entre si, sendo que o propósito de um algoritmo de agrupamento particional consiste em encontrar uma partição com $k$ grupos em uma base de dados com $N$ objetos. O agrupamento hierárquico, por sua vez, é uma sequência aninhada de agrupamentos particionais, cada um representando uma partição da base de dados em um número diferente de subconjuntos disjuntos. Em técnicas com sobreposição, procuram-se partições de dados nas quais um determinado 
objeto apresenta uma probabilidade ou grau de pertinência para todos os grupos. Algoritmos de agrupamento fuzzy (Yang, 1993; Babuska, 1998; Hoppner et al., 1999; Baraldi \& Blonda, 1999a,b), que constituem o foco de interesse do presente trabalho, pertencem a essa última categoria.

Quando os objetos contidos na base de dados, $\mathbf{X}=\left\{\mathbf{x}_{1}, \ldots, \mathbf{x}_{N}\right\}$, são representados por vetores $n$-dimensionais de atributos, i.e., $\mathbf{x}_{j}=\left[x_{j 1}, \ldots, x_{j n}\right]^{T} \in \mathbb{R}^{n}$, a base de dados $\mathbf{X}$ é chamada de matriz de dados. Na Tabela 2.1 é ilustrado um exemplo de matriz de dados contendo quatro objetos descritos por três atributos (i.e. $\mathbf{X}=\left\{\mathbf{x}_{1}, \mathbf{x}_{2}, \mathbf{x}_{3}, \mathbf{x}_{4}\right\}$, $\left.\mathbf{x}_{i} \in \mathbb{R}^{3} \forall i\right)$. Neste exemplo, $\mathbf{x}_{1}=\left[\begin{array}{lll}1 & 0 & 2\end{array}\right]^{T}, \mathbf{x}_{2}=\left[\begin{array}{lll}3 & 1 & 0\end{array}\right]^{T}, \mathbf{x}_{3}=\left[\begin{array}{lll}5 & 0 & 2\end{array}\right]^{T}$ e $\mathbf{x}_{4}=\left[\begin{array}{lll}3 & 4 & 1\end{array}\right]^{T}$.

$\mathbf{X}=$\begin{tabular}{|c|c|c|c|}
\hline & Atributo 1 & Atributo 2 & Atributo 3 \\
\hline Objeto 1 & 1 & 0 & 2 \\
\hline Objeto 2 & 3 & 1 & 0 \\
\hline Objeto 3 & 5 & 0 & 2 \\
\hline Objeto 4 & 3 & 4 & 1 \\
\hline
\end{tabular}

Tabela 2.1: Exemplo de matriz de dados com quatro objetos descritos por três atributos.

Alguns algoritmos encontram partições sem utilizar informações dos atributos dos objetos. Tais algoritmos utilizam apenas uma matriz relacional dos dados contendo os valores de similaridade/dissimilaridade entre os objetos da base de dados. Para maiores detalhes, veja a Seção 2.2.6.

A partição encontrada pelo algoritmo de agrupamento pode ser representada por uma matriz de partição $\mathbf{U}(\mathbf{X})$ de tamanho $k \times N$. Especificamente, a matriz de partição $\mathbf{U}(\mathbf{X})$ é tal que $\mathbf{U}=\left[u_{i j}\right]_{k \times N}, i=1, \ldots, k$ e $j=1, \ldots, N$, em que $u_{i j}$ é o grau de pertinência do objeto $\mathbf{x}_{j}$ ao grupo $\mathbf{C}_{i}$. Uma partição dos dados é denominada rígida quando é uma coleção $\mathbf{C}=\left\{\mathbf{C}_{1}, \ldots, \mathbf{C}_{k}\right\}$ de $k$ subconjuntos não sobrepostos de dados tal que $\mathbf{C}_{1} \cup \mathbf{C}_{2} \cup \cdots \cup \mathbf{C}_{k}=\mathbf{X}, \mathbf{C}_{i} \neq \oslash$, e $\mathbf{C}_{i} \cap \mathbf{C}_{l}=\oslash$ para $i \neq l$. Tal partição rígida pode também ser descrita em termos da matriz de partição $\mathbf{U}=\left[u_{i j}\right]_{k \times N}$, na qual $u_{i j} \in\{0,1\}$ denota o grau de pertinência do $j$-ésimo objeto ao $i$-ésimo grupo, respeitando as seguintes condições necessárias:

$$
\begin{gathered}
0<\sum_{j=1}^{N} u_{i j}<N, i=1, \ldots, k \\
\sum_{i=1}^{k} u_{i j}=1, j=1, \ldots, N .
\end{gathered}
$$

Uma vez que $u_{i j} \in\{0,1\}$, a condição necessária em (2.1) garante que todo grupo deve conter ao menos um objeto e que nenhum grupo pode conter todos os objetos, enquanto que a condição em (2.2) garante que um objeto pertence exclusivamente a um único grupo. Formalmente, o espaço de partições rígidas para um dado conjunto $\mathbf{X}$ de objetos 
é o conjunto:

$$
\mathbf{M}_{h}=\left\{\mathbf{U} \in \mathbb{R}^{k \times N} \mid u_{i j} \in\{0,1\}, \forall i, j ; \sum_{i=1}^{k} u_{i j}=1, \forall j ; 0<\sum_{j=1}^{N} u_{i j}<N, \forall i\right\}
$$

Na Tabela 2.2 é ilustrada uma matriz de partição rígida de quatro objetos agrupados em dois grupos. Note que o Grupo 1 contém os Objetos 1 e 2, enquanto o Grupo 2 contém os Objetos 3 e 4, i.e., $u_{11}=u_{12}=1, u_{21}=u_{22}=0, u_{13}=u_{14}=0$ e $u_{23}=u_{24}=1$.

$\mathbf{U}=$\begin{tabular}{|l|c|c|c|c|}
\hline & Objeto 1 & Objeto 2 & Objeto 3 & Objeto 4 \\
\hline Grupo 1 & 1 & 1 & 0 & 0 \\
\hline Grupo 2 & 0 & 0 & 1 & 1 \\
\hline \hline
\end{tabular}

Tabela 2.2: Exemplo de matriz de partição rígida de quatro objetos e dois grupos.

Em técnicas fuzzy procuram-se partições $\mathbf{U}=\left[u_{i j}\right]_{k \times N}$, nas quais $u_{i j} \in[0,1]$ denota o grau de pertinência do $j$-ésimo objeto ao $i$-ésimo grupo, respeitando a condição necessária em (2.1). Formalmente, o espaço de partições fuzzy para um dado conjunto $\mathbf{X}$ de objetos é o conjunto:

$$
\mathbf{M}_{f}=\left\{\mathbf{U} \in \mathbb{R}^{k \times N} \mid u_{i j} \in[0,1], \forall i, j ; 0<\sum_{j=1}^{N} u_{i j}<N, \forall i\right\} .
$$

Uma vez que $u_{i j} \in[0,1]$, a condição necessária em (2.1) garante que não podem existir grupos que apresentem graus de pertinência nulos para todos os objetos simultaneamente. Na Tabela 2.3 é ilustrada uma matriz de partição fuzzy de quatro objetos agrupados em dois grupos. Note que o Grupo 1 contém os Objetos 1 e 2 com o máximo de pertinência possível (i.e., $u_{11}=u_{12}=1$ ) e o Objeto 3 com apenas 0.2 de pertinência (i.e., $u_{13}=0.2$ ), enquanto o Objeto 4 pode ser considerado não pertencente ao Grupo 1 (pois $u_{14}=0$ ). Note também que o Grupo 2 contém os Objetos 3 e 4 com o máximo de pertinência possível (i.e., $u_{23}=u_{24}=1$ ) e contém os Objetos 1 e 2 com 0.5 de pertinência (i.e., $\left.u_{21}=u 22=0.5\right)$.

$\mathbf{U}=$\begin{tabular}{|c|c|c|c|c|}
\hline & Objeto 1 & Objeto 2 & Objeto 3 & Objeto 4 \\
\hline Grupo 1 & 1 & 1 & 0.2 & 0 \\
\hline Grupo 2 & 0.5 & 0.5 & 1 & 1 \\
\hline \hline
\end{tabular}

Tabela 2.3: Exemplo de matriz de partição fuzzy de quatro objetos e dois grupos.

Um caso particular de partições fuzzy dos dados são partições fuzzy probabilísticas, que procuram por partições $\mathbf{U}=\left[u_{i j}\right]_{k \times N}$, com $u_{i j} \in[0,1]$, respeitando as condições necessárias em (2.1) e (2.2). Formalmente, o espaço de partições fuzzy probabilísticas 
para um dado conjunto $\mathbf{X}$ de objetos é o conjunto:

$$
\mathbf{M}_{f p}=\left\{\mathbf{U} \in \mathbb{R}^{k \times N} \mid u_{i j} \in[0,1], \forall i, j ; \sum_{i=1}^{k} u_{i j}=1, \forall j ; 0<\sum_{j=1}^{N} u_{i j}<N, \forall i\right\}
$$

Uma vez que $u_{i j} \in[0,1]$, a condição necessária em (2.1) garante que não podem existir grupos que apresentem graus de pertinência nulos para todos os objetos simultaneamente, enquanto que a condição em (2.2) possibilita a interpretação de probabilidades aos elementos $u_{i j}$ (i.e., um determinado objeto, $\mathbf{x}_{j}$, apresenta uma probabilidade de pertencer ao grupo $\left.\mathbf{C}_{i}, i=1, \ldots, k\right)$. Na Tabela 2.4 é ilustrada uma matriz de partição fuzzy probabilística de quatro objetos agrupados em dois grupos. Note que o Grupo 1 contém o Objeto 1 com o máximo de pertinência possível (i.e., $u_{11}=1$ ), contém o Objeto 2 com pertinência 0.9 (i.e., $u_{12}=0.9$ ). Pode-se interpretar que o Objeto 2 apresenta mais características do Grupo 1 em comparação com o Grupo 2. O Grupo 1 ainda contém o Objeto 3 com pertinência de 0.5 , i.e., $u_{13}=0.5$ e o Objeto 4 com pertinência nula, i.e., $u_{14}=0$. O Grupo 2 contém o Objeto 4 com o máximo de pertinência possível (i.e., $u_{24}=1$ ), o Objeto 2 com pertinência 0.1 , o Objeto 3 com pertinência 0.5 e o Objeto 1 com pertinência nula, i.e., $u_{22}=0.1, u_{23}=0.5$ e $u_{21}=0$.

$\mathbf{U}=$\begin{tabular}{|l|c|c|c|c|}
\hline & Objeto 1 & Objeto 2 & Objeto 3 & Objeto 4 \\
\hline Grupo 1 & 1 & 0.9 & 0.5 & 0 \\
\hline Grupo 2 & 0 & 0.1 & 0.5 & 1 \\
\hline \hline
\end{tabular}

Tabela 2.4: Exemplo de matriz de partição fuzzy probabilística de quatro objetos e dois grupos.

De fato, partições rígidas podem ser vistas como um caso especial das partições fuzzy (i.e., $\mathbf{M}_{h} \subset \mathbf{M}_{f p} \subset \mathbf{M}_{f}$ ), no qual cada objeto recebe um grau de pertinência igual a 1 para o grupo ao qual o objeto pertence e um grau de pertinência igual a 0 para os demais grupos. Uma vez que uma partição fuzzy é obtida, é fácil determinar uma partição rígida substituindo, para cada objeto, o seu maior valor de pertinência por 1 e as demais pertinências daquele objeto por 0. Contudo, quando os dados apresentam sobreposições por natureza, partições fuzzy podem prover informações mais precisas.

\subsection{Algoritmos de Agrupamento Fuzzy de Dados}

Quando um algoritmo de agrupamento fuzzy é aplicado a uma base de dados com $N$ objetos $\mathbf{X}=\left\{\mathbf{x}_{1}, \ldots, \mathbf{x}_{N}\right\}$, o resultado final é uma matriz de partição fuzzy desses objetos em um dado número $k$ de grupos, tal que $\mathbf{U}=\left[u_{i j}\right]_{k \times N}$, no qual $\mathbf{U}$ é uma matriz de partição fuzzy $k \times N$ cujos elementos $u_{i j}$ representam o grau de pertinência (ou simplesmente pertinência) do $j$-ésimo objeto ao $i$-ésimo grupo fuzzy. 
A maioria dos algoritmos de agrupamento fuzzy de dados buscam minimizar alguma variação ou extensão da seguinte função objetivo:

$$
J=\sum_{j=1}^{N} \sum_{i=1}^{k}\left(u_{i j}\right)^{m} D_{i j}
$$

com a restrição $u_{i j} \in[0,1] \mathrm{e}$

$$
\sum_{i=1}^{k} u_{i j}=1, \quad 1 \leq j \leq N,
$$

i.e., $\mathbf{U}=\left[u_{i j}\right] \in \mathbf{M}_{f p}$ conforme definido em $(2.5)$, em que $m \in(1, \infty)$ é um expoente de fuzzificação (usualmente $m=2$ ), $D_{i j}$ é uma dada distância entre o $j$-ésimo objeto e o $i$-ésimo grupo, e $J$ é uma medida de dissimilaridade intra-grupo. Note que ao minimizar $J$ em (2.6) as pertinências $u_{i j}$ de um determinado j-ésimo objeto aos grupos mais próximos (i.e. valores baixos de $D_{i j}$ ) tendem a ser maiores, enquanto que as pertinências aos grupos mais distantes (i.e. valores altos de $D_{i j}$ ) tendem a ser menores. As diferenças entre aqueles algoritmos que minimizam exatamente essa função consistem na definição das distâncias $D_{i j}$ e das variáveis a serem consideradas no processo de minimização.

O método mais popular de solucionar (2.6) consiste em aplicar a interação de ponto fixo (Picard iteration - em inglês) com as condições de primeira ordem para pontos estacionários de (2.6) (Babuska, 1998). Assim, considerando $D_{i j}$ como constantes e $u_{i j}$ como variáveis, encontra-se que uma condição necessária é ${ }^{1}$ :

$$
u_{i j}=\left(\sum_{c=1}^{k}\left(\frac{D_{i j}}{D_{c j}}\right)^{1 /(m-1)}\right)^{-1}, 1 \leq i \leq k, 1 \leq j \leq N .
$$

Contudo, considerando $u_{i j}$ como constante, as outras condições necessárias dependem da definição de $D_{i j}$ e, portanto, são apresentadas nas próximas seções com os diferentes algoritmos associados a elas.

\subsubsection{FCM: Fuzzy c-Means}

O algoritmo FCM (Fuzzy c-Means - em inglês) (Dunn, 1973; Bezdek, 1981) é um dos mais facilmente compreendidos, bem documentados e intensamente utilizados na literatura. Ele consiste na versão fuzzy de um dos algoritmos de agrupamento mais conhecidos e estudados, denominado k-Médias (k-means — em inglês) (McQueen, 1967; Jain \& Dubes, 1988; Jain, 2010), considerado um dos dez algoritmos mais influentes em mineração de dados (Wu et al., 2008). O resultado final do algoritmo FCM consiste em uma matriz de partição $\mathbf{U}=\left[u_{i j}\right]_{k \times N} \in \mathbf{M}_{f p}$ contendo o grau de pertinência do objeto $\mathbf{x}_{j}$ ao grupo

\footnotetext{
${ }^{1}$ As técnicas matemáticas envolvidas para encontrar as condições necessárias não serão apresentadas neste documento. As condições necessárias são encontradas basicamente usando multiplicadores de Lagrande e igualando o gradiente à zero. Para mais detalhes veja (Bezdek, 1981; Hoppner et al., 1999) e as referências de cada algoritmo nas seções subsequentes.
} 
$\mathbf{C}_{i}$. O algoritmo minimiza $(2.6)$ em que $(1 \leq i \leq k, 1 \leq j \leq N)$

$$
D_{i j}=\left\|\mathbf{x}_{j}-\mathbf{v}_{i}\right\|_{\mathbf{A}}^{2}=\left(\mathbf{x}_{j}-\mathbf{v}_{i}\right)^{T} \mathbf{A}\left(\mathbf{x}_{j}-\mathbf{v}_{i}\right)
$$

é qualquer quadrado de norma induzida por produto interno (e.g. distância Euclideana quadrada) entre o $j$-ésimo objeto e o $i$-ésimo protótipo, e $\mathbf{v}_{i}$ é o protótipo do $i$-ésimo grupo (protótipo do grupo $\mathbf{C}_{i}$ ). Note que a matriz de norma induzida $\mathbf{A}$ (uma matriz definida positiva $n \times n$ ) usada em (2.9) define o formato (hiperelíptico) do grupo e deve ser definida a priori pelo usuário. Entretanto, na maioria dos cenários, o usuário não sabe a priori os formatos dos grupos presentes nos dados. Assim, a matriz A é comumente determinada como uma matriz identidade $\mathbf{I}_{n \times n}$ e, como consequência, $D_{i j}$ é definida como a distância Euclideana quadrada, o que induz grupos hiperesféricos.

Com tais definições, considerando $u_{i j}$ constante, uma condição necessária para minimizar (2.6) é dada por $(1 \leq i \leq k)$ :

$$
\mathbf{v}_{i}=\frac{\sum_{j=1}^{N}\left(u_{i j}\right)^{m} \mathbf{x}_{j}}{\sum_{j=1}^{N}\left(u_{i j}\right)^{m}} .
$$

O algoritmo FCM consiste, então, em dois passos principais (atualização das pertinências de acordo com (2.8) e atualização dos protótipos de acordo com (2.10)) que são executados alternadamente até a convergência do algoritmo. O procedimento completo para minimizar (2.6) com $D_{i j}$ definida em (2.9) é descrito no Algoritmo 1.

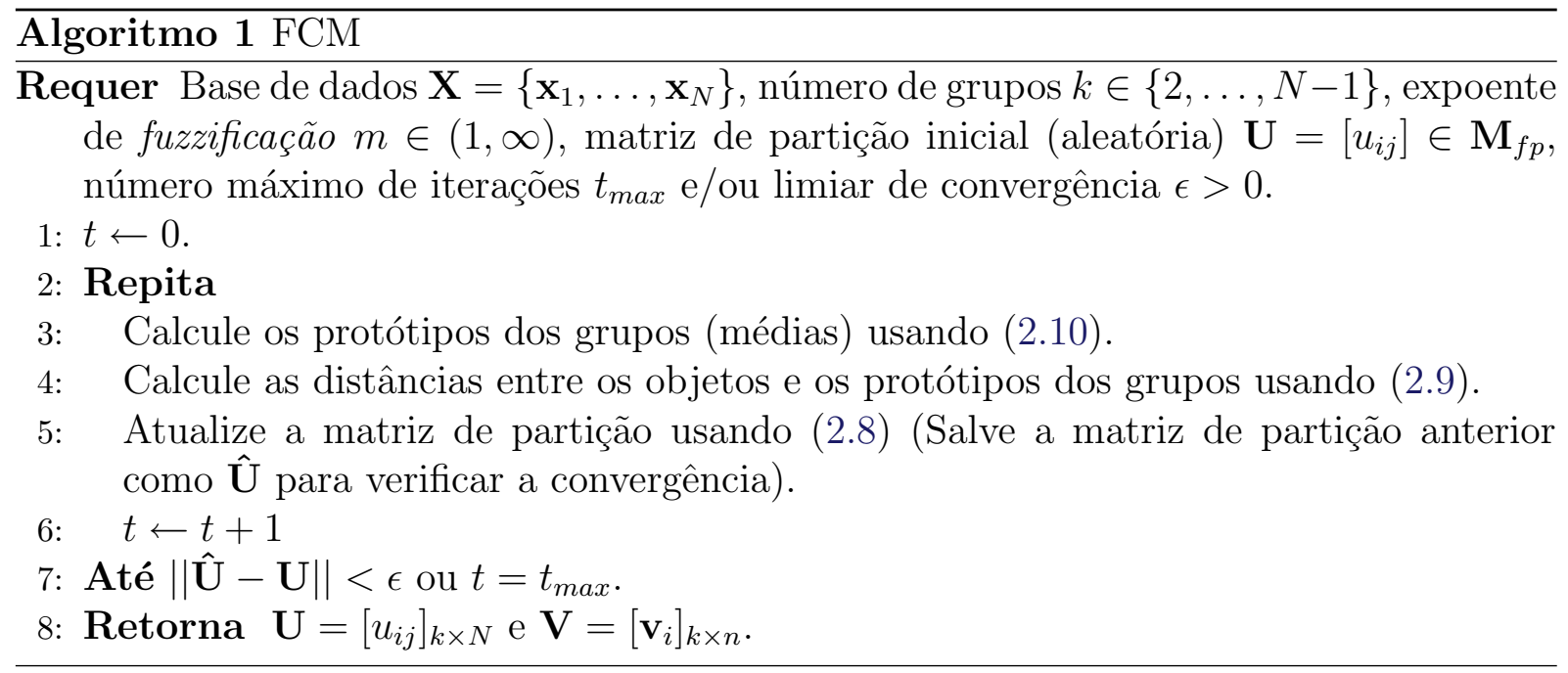

Existem algumas observações importantes que também permanecerão para os outros algoritmos descritos neste documento:

1. A matriz de partição inicial (U) requirida pelo Algoritmo 1 pode ser substituída pelos protótipos iniciais $\left(\mathbf{V}=\left[\mathbf{v}_{i}\right]_{k \times n} \in \mathbb{R}^{k \times n}\right)$. Para isso, o Passo 3 do Algoritmo 1 deve ser executado após o Passo 5 em cada iteração.

2. O critério de convergência $\|\hat{\mathbf{U}}-\mathbf{U}\|$ (comparação entre as matrizes de partição anterior e atual) pode usar qualquer norma (e.g. max) e pode ser substituída por 
outro critério (e.g., $\|\hat{\mathbf{V}}-\mathbf{V}\|$ - comparação entre as matrizes de protótipos anterior e atual).

3. O passo 5 do Algoritmo 1 requer que $D_{i j}>0$ para todo $i \in\{1, \ldots, k\}$ e $j \in$ $\{1, \ldots, N\}$. Para cada $j$, se $D_{i j}=0$ para $i \in I \subseteq\{1, \ldots, k\}$, então defina $u_{i j}$ de forma que: a) $u_{i j}=0$ para $i \notin I$; e b) $\sum_{i \in I} u_{i j}=1$. Em outras palavras, se um ou mais protótipos estiverem sobre um determinado objeto, então a pertinência desse objeto aos demais grupos deve ser nula, enquanto a pertinência desse objeto aos grupos cujos protótipos estão sobre ele deve somar 1.

\section{Análise de Complexidade - Tempo}

O algoritmo FCM consiste basicamente nos cálculos das pertinências e dos protótipos. Esse processo é repetido $t$ vezes $^{2}$. O cálculo de todos os protótipos em (2.10) requer $O(k N n)$ operações. O cálculo das distâncias entre todos os objetos e grupos em (2.9) requer $O\left(k N n^{2}\right)$ operações no caso mais geral, mas se a matriz de norma induzida $\mathbf{A}$ for a matriz identidade, essa complexidade é dada por $O(k N n)$. O cálculo das pertinências de todos os objetos e grupos em (2.8) requer $O\left(k^{2} N\right)$ operações com o método mais simples. Contudo, é possível melhorar essa complexidade de tal forma a requerer apenas $O(k N)$ operações (Kolen \& Hutcheson, 2002). Dessa forma, a complexidade de tempo do algoritmo de agrupamento FCM é $O\left(t\left(k N n+k N n^{2}+k N\right)\right) \rightarrow O\left(t k N n^{2}\right)$, que se reduz a $O(t k N n)$ se a norma Euclideana é usada.

\section{Análise de Complexidade - Espaço}

O primeiro requisito do algoritmo de agrupamento é a base de dados $\mathbf{X}$, que requer $O(N n)$ unidades de espaço. As saídas do algoritmo FCM também são armazenadas, o que requer $O(k N)$ para a matriz de partição e $O(k n)$ para os protótipos dos grupos. Assumimos também que as distâncias entre cada objeto e cada grupo é calculada e armazenada em uma tabela separada, o que requer $O(k N)$ unidades de espaço. Para calcular as distâncias usando (2.9) é necessário armazenar a matriz de norma induzida ${ }^{3} \mathbf{A}$ que requer $O\left(n^{2}\right)$. Dado que $N>k$, a complexidade de espaço do algoritmo de agrupamento FCM é $O\left(N n+k N+k n+k N+n^{2}\right) \rightarrow O\left(N n+k N+k n+n^{2}\right) \rightarrow O\left(N n+k N+n^{2}\right)$, que se reduz a $O(N n+k N)$ quando a norma Euclideana é usada.

É importante deixar claro que para a análise de complexidade de espaço foi considerado que todos os dados necessários, bem como as saídas dos algoritmos, são armazenados em memória principal. Dessa forma, nenhum acesso em dispositivos de memória secundária é necessário. Portanto, foi considerado que o tempo necessário para acessar e escrever os dados não afetam a análise de complexidade de tempo.

\footnotetext{
${ }^{2} t_{\max }$ vezes no pior dos casos quando $t_{\max }$ é dado como entrada para o algoritmo.

${ }^{3}$ Note que se a matriz A for a matriz identidade, então não é necessário armazená-la.
} 


\subsubsection{Algoritmos que Buscam por Grupos Hiperelípticos}

O algoritmo FCM apresentado anteriormente na Seção 2.2.1 adota uma norma fixa como medida de distância durante sua execução. Comumente, a distância Euclideana é adotada e, portanto, o algoritmo tende a induzir grupos hiperesféricos. Nesta seção são apresentados alguns algoritmos que utilizam diferentes medidas de distância com o objetivo de encontrar grupos com formatos hiperelípticos.

\subsubsection{GK: Gustafson-Kessel}

O algoritmo FCM descrito na Seção 2.2.1 tende a induzir grupos hiperesféricos porque utiliza a distância Euclideana como medida de dissimilaridade entre objetos e protótipos. Para adicionar o efeito de diferentes formas nos grupos, em particular grupos hiperelípticos (possivelmente hiperesféricos), Gustafson \& Kessel (1978) generalizaram a função objetivo do FCM com a adição de matrizes de covariância (Gustafson \& Kessel, 1978). A partir desse princípio, (2.6) é minimizada (com respeito a $u_{i j}, \mathbf{v}_{i}$ e $\mathbf{A}_{i}$ ) com as distâncias definidas por $(1 \leq i \leq k, 1 \leq j \leq N)$ :

$$
D_{i j}=\left\|\mathbf{x}_{j}-\mathbf{v}_{i}\right\|_{\mathbf{A}_{i}}^{2}=\left(\mathbf{x}_{j}-\mathbf{v}_{i}\right)^{T} \mathbf{A}_{i}\left(\mathbf{x}_{j}-\mathbf{v}_{i}\right) .
$$

Nesse caso, fixando $u_{i j}$ e $\mathbf{A}_{i}$, e considerando $\mathbf{v}_{i}$ como variáveis, as condições necessárias para minimizar (2.6) também levam a (2.10). Considerando $\mathbf{A}_{i}$ como variáveis, devido ao fato de que (2.6) é linear em relação a $\mathbf{A}_{i}$, o valor da função objetivo pode ser tão pequeno quanto desejado, fazendo com que $\mathbf{A}_{i}$ seja menos positiva definida. Para evitar esse comportamento, $\mathbf{A}_{i}$ é limitada de tal forma que $\operatorname{det}\left(\mathbf{A}_{i}\right)=\rho_{i}$ (usualmente $\rho_{i}=1 \forall i$ ) e, consequentemente, limita o volume do grupo correspondente. As condições necessárias são então dadas por $(1 \leq i \leq k)$ :

$$
\begin{gathered}
\mathbf{A}_{i}=\left(\rho_{i} \cdot \operatorname{det}\left(\mathbf{F}_{i}\right)\right)^{1 / n}\left(\mathbf{F}_{i}\right)^{-1} \\
\mathbf{F}_{i}=\frac{\sum_{j=1}^{N}\left(u_{i j}\right)^{m}\left(\mathbf{x}_{j}-\mathbf{v}_{i}\right)\left(\mathbf{x}_{j}-\mathbf{v}_{i}\right)^{T}}{\sum_{j=1}^{N}\left(u_{i j}\right)^{m}}
\end{gathered}
$$

O procedimento completo para minimizar (2.6) com as distâncias definidas por (2.11) é descrito no Algoritmo 2.

\section{Análise de Complexidade - Tempo}

O cálculo de todos os protótipos em (2.10) requer $O(k N n)$ operações. O cálculo de $\mathbf{A}_{i}$ em (2.12) requer $O\left(k n^{3}\right)$ operações e o cálculo das matrizes de covariâncias em (2.13) requer $O\left(k N n^{2}\right)$ operações. O cálculo das distâncias entre os objetos e os grupos em (2.11) requer $O\left(k N n^{2}\right)$ operações. A atualização das pertinências em (2.8) requer $O(k N)$ operações, usando o mesmo procedimento adotado por Kolen \& Hutcheson (2002). 


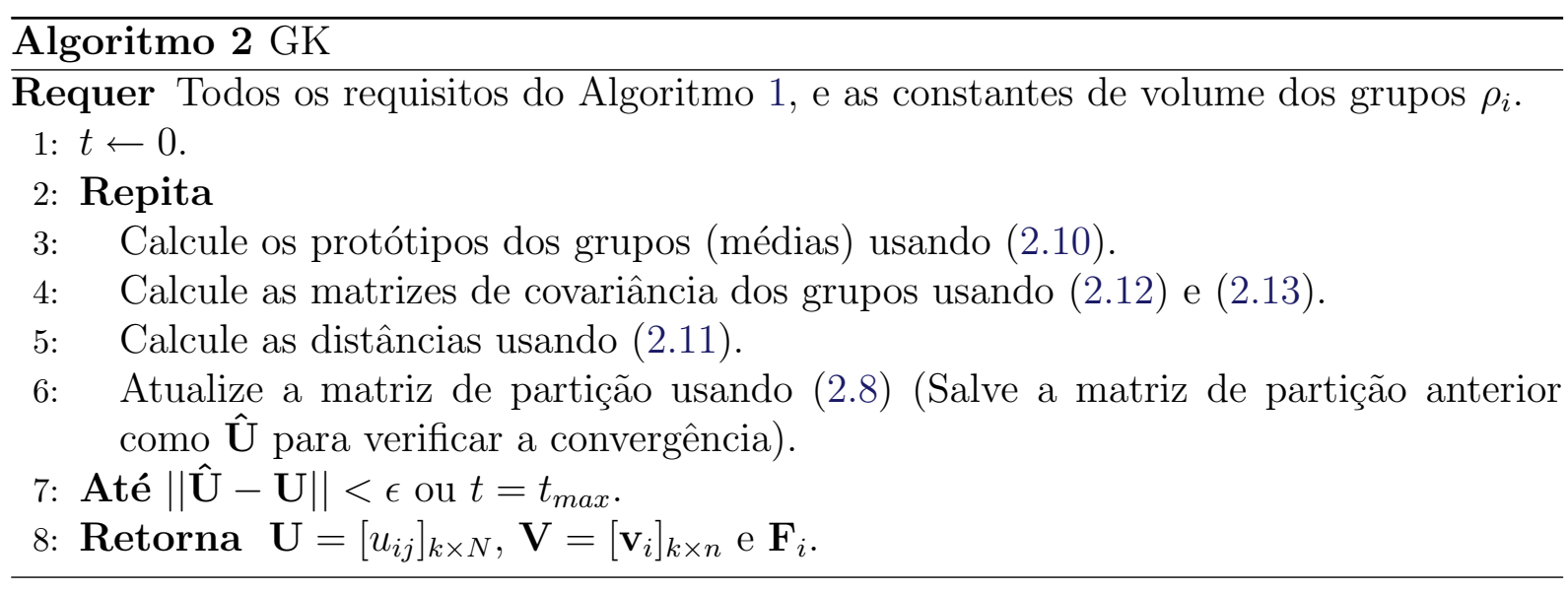

Portanto, a complexidade de tempo do algoritmo de agrupamento GK é $O\left(t\left(k N n+k n^{3}+\right.\right.$ $\left.\left.k N n^{2}+k N n^{2}+k N\right)\right) \rightarrow O\left(t k n^{3}+t k n^{2} N\right)$.

\section{Análise de Complexidade - Espaço}

A base de dados $\mathbf{X}$ requer $O(N n)$ unidades de espaço. As saídas do algoritmo requerem $O(k N)$ para a matriz de partição, $O(k n)$ para os protótipos dos grupos, e $O\left(k n^{2}\right)$ para as matrizes de covariância. As distâncias são calculadas e armazenadas em uma tabela à parte, o que requer $O(k N)$ unidades de espaço. Para o cálculo das distâncias usando (2.11) é necessário armazenar $\mathbf{A}_{i}$ em (2.12) e as matrizes de covariância em (2.13), o que requer $O\left(k n^{2}\right)$. Portanto, a complexidade de espaço do algoritmo de agrupamento GK é $O\left(N n+k N+k n+k n^{2}+k N+k n^{2}\right) \rightarrow O\left(N n+k N+k n^{2}\right)$.

\subsubsection{GG: Gath and Geva}

O algoritmo de agrupamento GG (Gath e Geva) (Bezdek \& Dunn, 1975; Gath \& Geva, 1989) também induz grupos hiperelípticos. O algoritmo utiliza uma distância que não é linear com respeito às matrizes de covariâncias (Bezdek \& Dunn, 1975) e, portanto, os grupos não precisam ser limitados em seu volume. Nesse caso, (2.6) é minimizada com as distâncias definidas por $(1 \leq i \leq k, 1 \leq j \leq N)$ :

$$
D_{i j}=\frac{\left[\operatorname{det}\left(\mathbf{F}_{i}\right)\right]^{1 / 2}}{P_{i}} \exp \left[\frac{1}{2}\left(\mathbf{x}_{j}-\mathbf{v}_{i}\right)^{T} \mathbf{F}_{i}^{-1}\left(\mathbf{x}_{j}-\mathbf{v}_{i}\right)\right]
$$

em que $\mathbf{F}_{i}$ denota a matriz de covariância do grupo $\mathbf{C}_{i}$, dada por (2.13) (Hoppner et al., 1999) e $P_{i}$ é a probabilidade a priori de selecionar o grupo $\mathbf{C}_{i}$, dada por (Hoppner et al., 1999) $(1 \leq i \leq k)$ :

$$
P_{i}=\frac{\sum_{j=1}^{N}\left(u_{i j}\right)^{m}}{\sum_{j=1}^{N} \sum_{c=1}^{k}\left(u_{c j}\right)^{m}} .
$$

Note que, ao contrário do algoritmo de agrupamento GK, não existe uma constante de volume para cada grupo e, portanto, esse algoritmo é mais apto a detectar grupos de 
formas hiperelípticas com diferentes tamanhos e densidades (Gath \& Geva, 1989). Em contrapartida, a distância utilizada envolve um termo exponencial e, dessa forma, decresce mais rapidamente que a norma induzida por produto interno. Isso faz com que o algoritmo GG encontre partições quase rígidas $\left(\forall j, \exists i\right.$ tal que $u_{i j} \approx 1$ ) e, consequentemente, seja mais sensível às condições iniciais.

Novamente, minimizar (2.6) com respeito a $\mathbf{v}_{i}$ leva a (2.10). O procedimento completo para minimizar (2.6) com as distâncias definidas por (2.14) é descrito no Algoritmo 3.

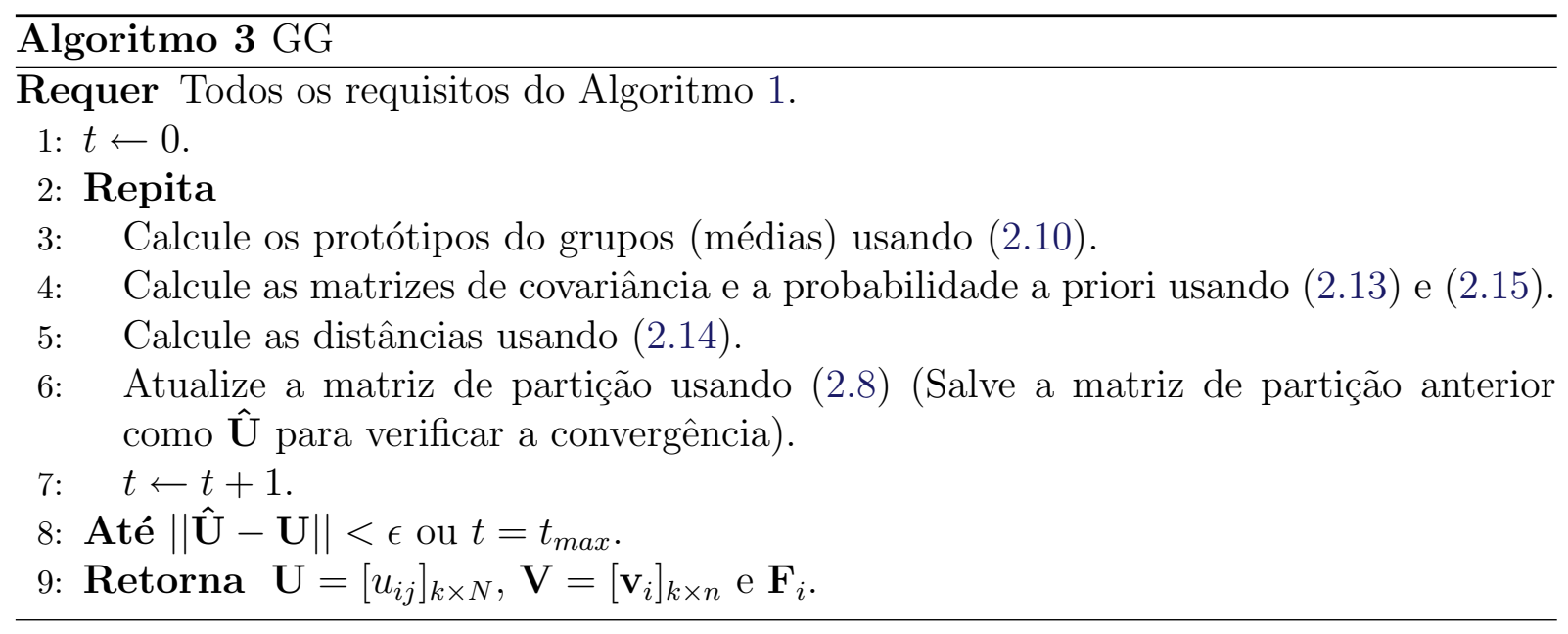

\section{Análise de Complexidade - Tempo}

O cálculo dos protótipos em (2.10) requer $O(k N n)$ operações. O cálculo das matrizes de covariância em (2.13) e a probabilidade a priori em (2.15) requerem $O\left(k N n^{2}\right)$ e $O(k N)$ operações, respectivamente. O cálculo das distâncias em (2.14) requer $O\left(k N n^{2}+k n^{3}\right)$ operações (calculando $\mathbf{F}^{-1}$ e $\operatorname{det}\left(\mathbf{F}_{i}\right)$ separadamente para cada grupo). A atualização das pertinências em (2.8) requer $O(k N)$ operações, usando o procedimento adotado por Kolen \& Hutcheson (2002). Portanto, a complexidade de tempo do algoritmo de agrupamento GG é $O\left(t\left(k N n+k N n^{2}+k N+k N n^{2}+k n^{3}+k N\right)\right) \rightarrow O\left(t k n^{3}+t k n^{2} N\right)$.

\section{Análise de Complexidade - Espaço}

A base de dados $\mathbf{X}$ requer $O(N n)$ unidades de espaço. As saídas do algoritmo requerem $O(k N)$ para a matriz de partição, $O(k n)$ para os protótipos e $O\left(k n^{2}\right)$ para as matrizes de covariância. As distâncias são calculadas e armazenadas em uma tabela à parte, o que requer $O(k N)$ unidades de espaço. Para o cálculo das distâncias usando (2.14) é necessário armazenar as matrizes de covariância em (2.13), o que requer $O\left(k n^{2}\right)$. Portanto, a complexidade de espaço do algoritmo de agrupamento GG é $O\left(N n+k N+k n+k n^{2}+\right.$ $\left.k N+k n^{2}\right) \rightarrow O\left(N n+k N+k n^{2}\right)$. 


\subsubsection{Algoritmos que Utilizam Protótipos Não Pontuais}

Os algoritmos apresentados nas seções anteriores adotam protótipos pontuais. Mais especificamente, o protótipo é representado por um ponto médio do grupo. Dessa forma, as pertinências dos objetos aos grupos são calculadas considerando as distâncias (sendo que cada algoritmo utiliza uma distância específica) desses objetos a tais pontos médios. Nesta seção são apresentados alguns algoritmos que adotam protótipos com diferentes formatos.

\subsubsection{FCV: Fuzzy c-Varieties}

Diferentemente dos algoritmos anteriores, o algoritmo FCV (Fuzzy c-Varieties - em inglês) (Bezdek et al., 1981a) mede as distâncias entre os objetos e variedades lineares $r$-dimensionais (i.e. linhas quando $r=1$, planos quando $r=2$ e hiperplanos quando $2<r<n)$. Portanto, esse algoritmo foi desenvolvido para o reconhecimento de grupos formados por linhas, planos ou hiperplanos, que podem ser úteis na área de processamento de imagens para a identificação de bordas em reconhecimento de imagens (Hoppner et al., 1999). O algoritmo minimiza (2.6) com as distâncias definidas por $(1 \leq i \leq k, 1 \leq j \leq N)$ :

$$
\begin{aligned}
D_{i j} & =\left\|\mathbf{x}_{j}-\mathbf{v}_{i}\right\|_{\mathbf{A}}^{2}-\sum_{l=1}^{r}<\mathbf{x}_{j}-\mathbf{v}_{i}, \mathbf{s}_{i l}>_{\mathbf{A}}^{2} \\
& =\left(\mathbf{x}_{j}-\mathbf{v}_{i}\right)^{T} \mathbf{A}\left(\mathbf{x}_{j}-\mathbf{v}_{i}\right)-\sum_{l=1}^{r}\left(\left(\mathbf{x}_{j}-\mathbf{v}_{i}\right)^{T} \mathbf{A}^{1 / 2} \mathbf{s}_{i l}\right)^{2}
\end{aligned}
$$

em que $\mathbf{v}_{i}$ é um ponto pelo qual a variedade passa, $\langle\cdot, \cdot\rangle$ denota um produto escalar e $\left(\mathbf{s}_{i 1}, \ldots, \mathbf{s}_{i r}\right)$ é uma $r$-tupla de vetores linearmente independentes que medem a variedade $v_{i r}$ e correspondem aos $r$ autovetores principais (autovetores normalizados correspondentes aos $r$ maiores autovalores) da $i$-ésima matriz de dispersão intra-grupo fuzzy $\mathbf{S}_{i}=\mathbf{A}^{1 / 2} \mathbf{F}_{i} \mathbf{A}^{1 / 2}$ (em que $\mathbf{F}_{i}$ é dado por (2.13)). Portanto, as variedades são definidas de acordo com o alongamento de seu grupo correspondente. As distâncias entre cada objeto e cada grupo são então influenciadas por quão próxima a variedade correspondente passa pelo objeto. Note que se $r=0$ então (2.16) se reduz a (2.9) e o algoritmo FCV se reduz ao algoritmo FCM.

Minimizar (2.6) usando a definição de distâncias em (2.16) com respeito a $\mathbf{v}_{i}$ também leva a (2.10). O procedimento completo é então ilustrado no Algoritmo 4.

\section{Análise de Complexidade - Tempo}

O cálculo dos protótipos em (2.10) requer $O(k N n)$ operações. O cálculo das matrizes de covariância em (2.13) e $\mathbf{S}_{i}$ requerem $O\left(k N n^{2}\right)$ operações. Obter os autovetores principais de $\mathbf{S}_{i}$ requer $O\left(k n^{3}\right)$. O cálculo das distâncias em (2.16) requer $O\left(k N n^{2} r\right)$ 


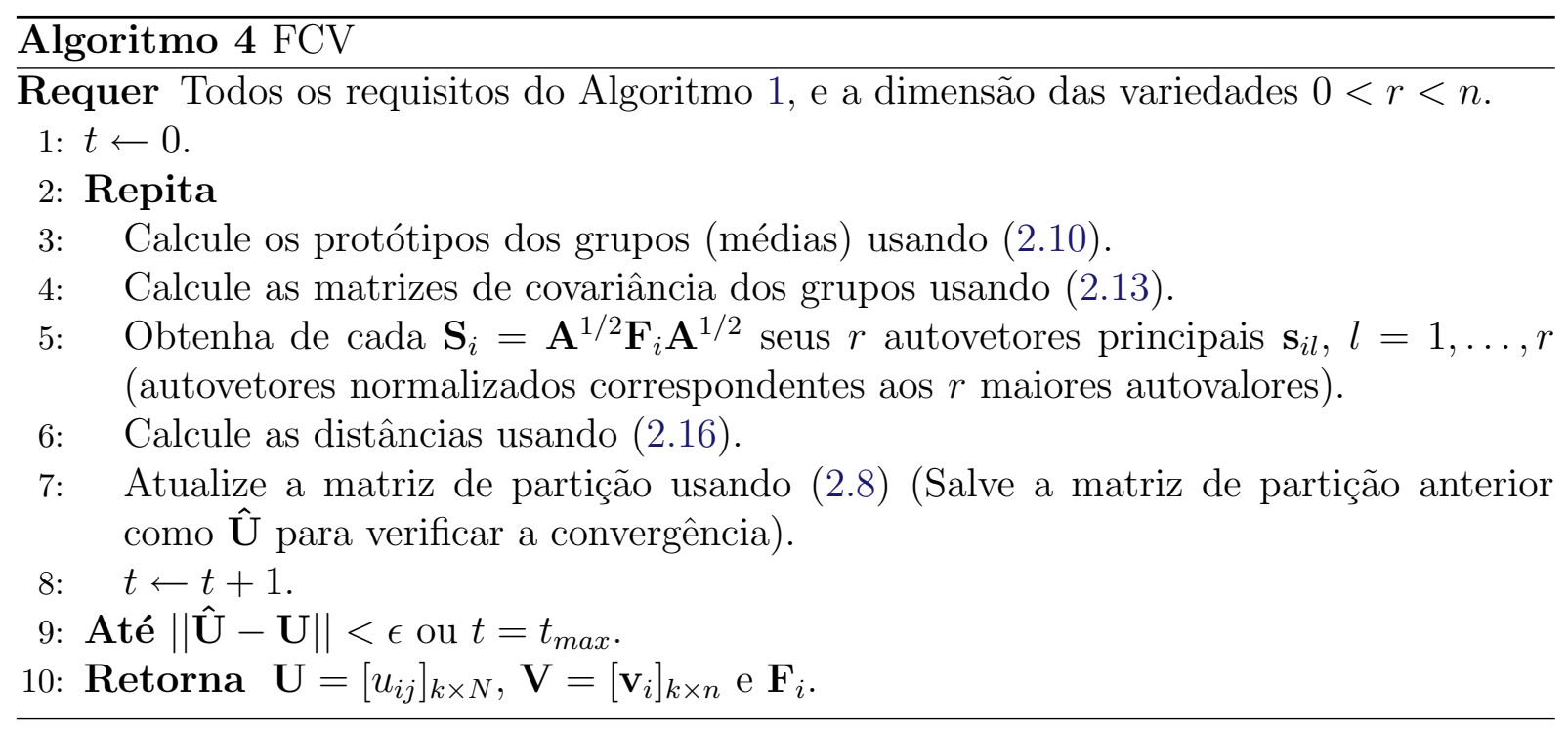

operações. A atualização das pertinências em (2.8) requer $O(k N)$ operações, usando o mesmo procedimento adotado por Kolen \& Hutcheson (2002). Portanto, a complexidade de tempo do algoritmo de agrupamento FCV é $O\left(t\left(k N n+k N n^{2}+k n^{3}+k N n^{2} r+k N\right)\right) \rightarrow$ $O\left(t k n^{3}+t k n^{2} N r\right)$.

\section{Análise de Complexidade - Espaço}

A base de dados $\mathbf{X}$ requer $O(N n)$ unidades de espaço. As saídas do algoritmo requerem $O(k N)$ para a matriz de partição, $O(k n)$ para os protótipos dos grupos, e $O\left(k n^{2}\right)$ para as matrizes de covariância. A matriz de norma induzida requer $O\left(n^{2}\right)$. As distâncias são calculadas e armazenadas em uma tabela à parte, o que requer $O(k N)$ unidades de espaço. Portanto, a complexidade de espaço do algoritmo de agrupamento FCV é $O\left(N n+k N+k n+k n^{2}+n^{2}+k N\right) \rightarrow O\left(N n+k N+k n^{2}\right)$.

\subsubsection{FCE: Fuzzy c-Elliptotypes}

O algoritmo FCV procura por grupos com formatos de retas, planos ou hiperplanos. Porém tais grupos são infinitos (e.g. uma reta é infinita em sua extensão) e podem "unir" grupos que estão naturalmente separados (Bezdek et al., 1981b; Hoppner et al., 1999; Babuska, 1998). Para evitar esse problema, o algoritmo FCE (Fuzzy c-Elliptotypes - em inglês) (Bezdek et al., 1981b) utiliza uma distância modificada, dada por uma combinação convexa de (2.9) e $(2.16)(1 \leq i \leq k, 1 \leq j \leq N)$ : 


$$
\begin{aligned}
D_{i j} & =(1-\alpha)\left\|\mathbf{x}_{j}-\mathbf{v}_{i}\right\|_{\mathbf{A}}^{2}+\alpha\left(\left\|\mathbf{x}_{j}-\mathbf{v}_{i}\right\|_{\mathbf{A}}^{2}-\sum_{l=1}^{r}<\mathbf{x}_{j}-\mathbf{v}_{i}, \mathbf{s}_{i l}>_{\mathbf{A}}^{2}\right) \\
& =\left\|\mathbf{x}_{j}-\mathbf{v}_{i}\right\|_{\mathbf{A}}^{2}-\alpha \sum_{l=1}^{r}<\mathbf{x}_{j}-\mathbf{v}_{i}, \mathbf{s}_{i l}>_{\mathbf{A}}^{2} \\
& =\left(\mathbf{x}_{j}-\mathbf{v}_{i}\right)^{T} \mathbf{A}\left(\mathbf{x}_{j}-\mathbf{v}_{i}\right)-\alpha \sum_{l=1}^{r}\left(\left(\mathbf{x}_{j}-\mathbf{v}_{i}\right)^{T} \mathbf{A}^{1 / 2} \mathbf{s}_{i l}\right)^{2}
\end{aligned}
$$

em que $0 \leq \alpha \leq 1$ define o alongamento das hiperelipsóides na direção dos vetores $\mathbf{s}_{i l}$. Quando $\alpha=0, D_{i j}$ se reduz a (2.9). Em contrapartida, se $\alpha=1$ então $D_{i j}$ se reduz a (2.16).

Novamente, minimizar (2.6) com respeito a $\mathbf{v}_{i}$ usando as distâncias definidas em (2.17) também leva a (2.10). O procedimento iterativo completo é descrito no Algoritmo 5.

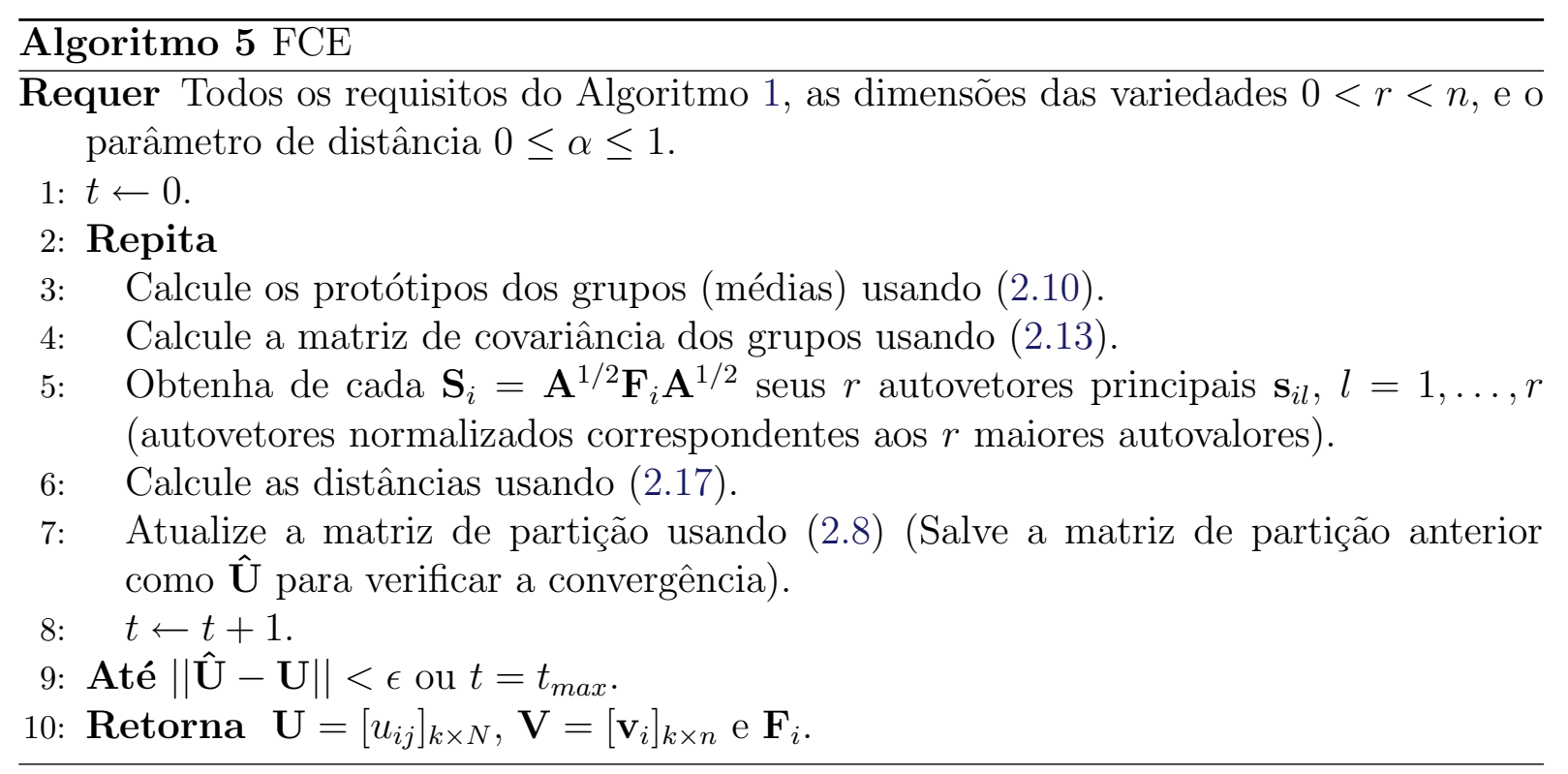

\section{Análise de Complexidade - Tempo}

O cálculo dos protótipos em (2.10) requer $O(k N n)$ operações. O cálculo das matrizes de covariância em (2.13) e $\mathbf{S}_{i}$ requerem $O\left(k N n^{2}\right)$ operações. Obter os autovetores principais de $\mathbf{S}_{i}$ requer $O\left(k n^{3}\right)$. O cálculo das distâncias em (2.17) requer $O\left(k N n^{2} r\right)$ operações. A atualização das pertinências em (2.8) requer $O(k N)$ operações, usando o mesmo procedimento adotado por Kolen \& Hutcheson (2002). Portanto, a complexidade de tempo do algoritmo de agrupamento FCE é $O\left(t\left(k N n+k N n^{2}+k n^{3}+k N n^{2} r+k N\right)\right) \rightarrow$ $O\left(t k n^{3}+t k n^{2} N r\right)$.

\section{Análise de Complexidade - Espaço}

A base de dados $\mathbf{X}$ requer $O(N n)$ unidades de espaço. As saídas do algoritmo requerem $O(k N)$ para a matriz de partição, $O(k n)$ para os protótipos dos grupos, e $O\left(k n^{2}\right)$ para 
as matrizes de covariância. A matriz de norma induzida requer $O\left(n^{2}\right)$. As distâncias são calculadas e armazenadas em uma tabela à parte, o que requer $O(k N)$ unidades de espaço. Portanto, a complexidade de espaço do algoritmo de agrupamento FCE é $O\left(N n+k N+k n+k n^{2}+n^{2}+k N\right) \rightarrow O\left(N n+k N+k n^{2}\right)$.

\subsubsection{EFCM: Extended Fuzzy c-Means}

O algoritmo FCM (Seção 2.2.1) utiliza protótipos pontuais. Dessa forma, o valor de pertinência dos objetos a um determinado grupo está relacionado com o quão próximo os objetos estão em relação ao protótipo (pontual). Mesmo objetos próximos a um protótipo podem apresentar uma pertinência baixa (e.g. se outro protótipo também estiver próximo). Entretanto, em muitas aplicações os objetos que estão próximos a um protótipo podem ser considerados completamente pertencentes ao grupo correspondente. Isso sugere que os protótipos dos grupos devem ser estendidos até certa distância de tais protótipos (Kaymak \& Setnes, 2002). Dessa forma, foi proposto o algoritmo EFCM (Extended Fuzzy c-Means - em inglês) (Kaymak \& Setnes, 2002) que utiliza protótipos com volumes. Os protótipos não são mais pontos no espaço, mas apresentam um volume fixo. Todos os objetos que se encontram "dentro" do protótipo possuem pertinência máxima ao grupo correspondente.

Formalmente, o algoritmo EFCM minimiza (2.6) com as distâncias dadas por $(1 \leq i \leq k, 1 \leq j \leq N)$ :

$$
D_{i j}=\left\|\mathbf{x}_{j}-\mathbf{v}_{i}\right\|_{\mathbf{A}}^{2}-r_{i}^{2}=\left(\mathbf{x}_{j}-\mathbf{v}_{i}\right)^{T} \mathbf{A}\left(\mathbf{x}_{j}-\mathbf{v}_{i}\right)-r_{i}^{2}
$$

em que $r_{i}^{2}$ especifica o volume do protótipo do grupo $\mathbf{C}_{i}$.

De maneira similar ao algoritmo FCM, minimizar (2.6) usando as distâncias definidas em (2.18) com respeito a $\mathbf{v}_{i}$ leva a (2.10). Minimizar (2.6) com respeito a $u_{i j}$ também leva a (2.8). Entretanto utilizar (2.8) com as distâncias definidas em (2.18) pode levar a pertinências negativas, o que é proibitivo, dado que $\mathbf{U} \in \mathbf{M}_{f p}{ }^{4}$. Note que um objeto $\mathbf{x}_{j}$ que se encontra dentro do raio de um determinado grupo $\mathbf{C}_{i}$ apresenta distância negativa $\left(D_{i j}<0\right)$ e, consequentemente, pode levar a uma pertinência $u_{i j}$ negativa ao grupo $\mathbf{C}_{i}$ usando (2.8). Para contornar o problema, Kaymak \& Setnes (2002) adicionaram a restrição de não negatividade aos elementos $u_{i j}$. Assim, considerando $D_{i j}$ (definido em (2.18)) como constante e $u_{i j}$ como variável com a restrição de não negatividade, encontrase que uma condição necessária para minimizar (2.6) é $(1 \leq i \leq k, 1 \leq j \leq N)$ :

$$
u_{i j}=\left(\sum_{c=1}^{k}\left(\frac{\hat{D}_{i j}}{\hat{D}_{c j}}\right)^{1 /(m-1)}\right)^{-1}
$$

\footnotetext{
${ }^{4}$ Apesar de considerar $\mathbf{U} \in \mathbf{M}_{f p}$, ao encontrar as condições necessárias em (2.8), a restrição $u_{i j} \geq 0$ não está diretamente presente nas técnicas matemáticas usadas. Entretanto, quando $D_{i j}>0 \forall i, j$ essa restrição é satisfeita. Porém, não é este o caso do algoritmo EFCM, no qual é possível que exista $D_{i j}<0$.
} 
em que $\hat{D}_{i j}$ é dada por:

$$
\hat{D}_{i j}=\max \left\{0, D_{i j}\right\}=\max \left\{0,\left(\mathbf{x}_{j}-\mathbf{v}_{i}\right)^{T} \mathbf{A}\left(\mathbf{x}_{j}-\mathbf{v}_{i}\right)-r_{i}^{2}\right\} .
$$

Para estimar o raio dos grupos, os autores do algoritmo propuseram usar uma informação de volume que pode ser obtida pela matriz de covariância fuzzy em (2.13). Assim, os raios são estimados por (Kaymak \& Setnes, 2002):

$$
r_{i}^{2}=\frac{\left(\operatorname{det}\left(\mathbf{F}_{i}\right)\right)^{1 / n}}{k^{2}} .
$$

Além do algoritmo EFCM, os autores propuseram de maneira semelhante o algoritmo EGK (versão do algoritmo GK, apresentado na Seção 2.2.2.1, desenvolvida para utilizar protótipos com volumes). Foi proposto também uma maneira do algoritmo estimar o número de grupos na base de dados, unindo grupos próximos a cada iteração do algoritmo. No Algoritmo 6 foi ilustrada apenas a versão estendida do FCM (Seção 2.2.1), denominada EFCM, considerando que o número de grupos $k$ é fixo. Mais detalhes sobre o algoritmo podem ser encontrados no trabalho de Kaymak \& Setnes (2002).

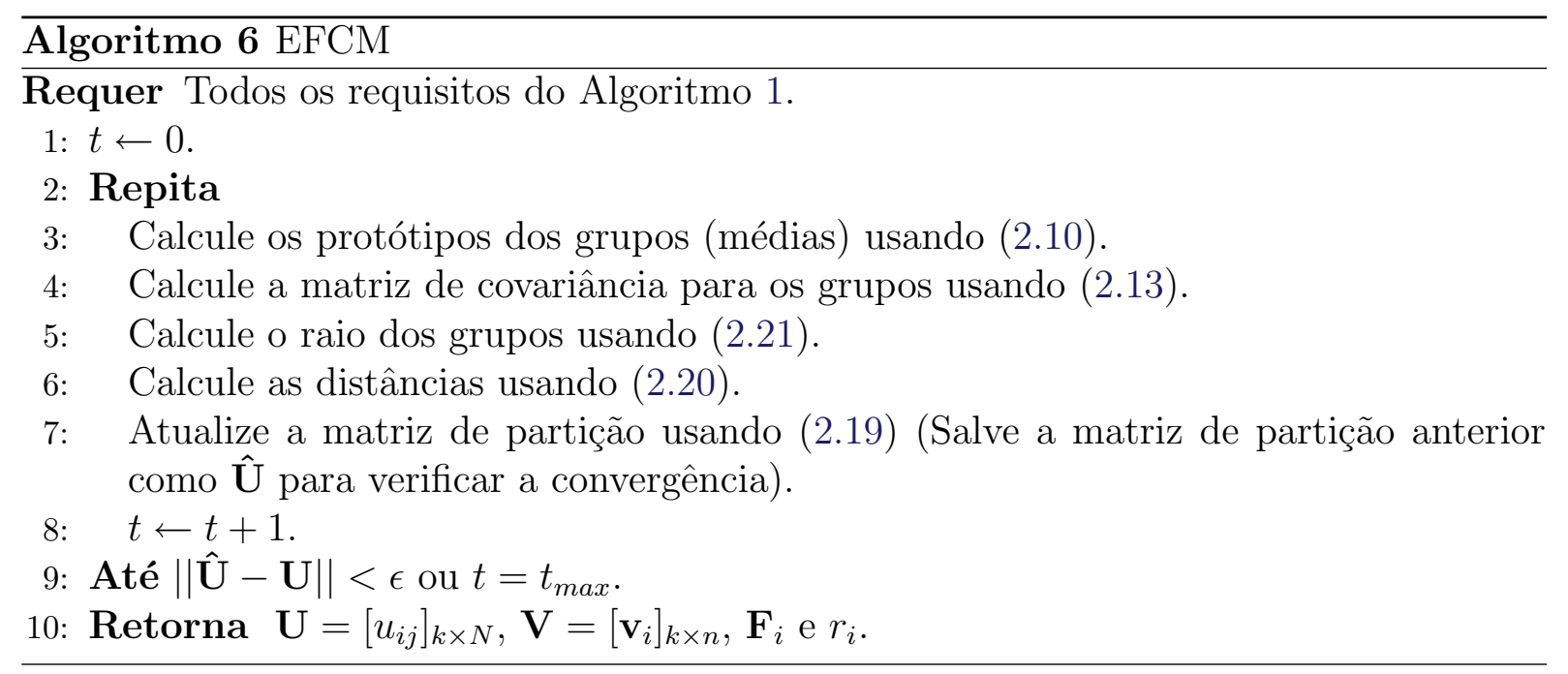

\section{Análise de Complexidade - Tempo}

O cálculo dos protótipos em (2.10) requer $O(k N n)$ operações. O cálculo das matrizes de covariância em (2.13) requer $O\left(k N n^{2}\right)$ operações. Obter os raios dos grupos em (2.21) requer $O\left(k n^{3}\right)$. O cálculo das distâncias em (2.20) requer $O\left(k N n^{2}\right)$ operações. A atualização das pertinências em (2.19) requer $O(k N)$ operações, usando o mesmo procedimento adotado por Kolen \& Hutcheson (2002). Portanto, a complexidade de tempo do algoritmo de agrupamento EFCM é $O\left(t\left(k N n+k N n^{2}+k n^{3}+k N n^{2}+k N\right)\right) \rightarrow O\left(t k n^{3}+t k n^{2} N\right)$.

\section{Análise de Complexidade - Espaço}

A base de dados $\mathbf{X}$ requer $O(N n)$ unidades de espaço. As saídas do algoritmo requerem $O(k N)$ para a matriz de partição, $O(k n)$ para os protótipos dos grupos, $O\left(k n^{2}\right)$ para as 
matrizes de covariância e $O(k)$ para os raios dos grupos. As distâncias são calculadas e armazenadas em uma tabela à parte, o que requer $O(k N)$ unidades de espaço. Portanto, a complexidade de espaço do algoritmo de agrupamento EFCM é $O(N n+k N+k n+$ $\left.k n^{2}+k+k N\right) \rightarrow O\left(N n+k N+k n^{2}\right)$.

\subsubsection{PLFC: Prototype-less Fuzzy Clustering}

O algoritmo PLFC (Prototype-less Fuzzy Clustering — em inglês) (Borgelt, 2007) encontra as pertinências dos objetos aos grupos sem utilizar qualquer protótipo. A ideia do algoritmo consiste em encontrar a matriz de partição $\mathbf{U}$ contendo as pertinências dos objetos a cada grupo, sem a necessidade de um passo intermediário para o cálculo dos protótipos. A função objetivo a ser minimizada agora não deve levar em consideração as distâncias entre objetos e protótipos. Borgelt (2007) definiu então a seguinte função objetivo:

$$
J=\frac{1}{2} \sum_{i=1}^{k} \sum_{j=1}^{N} \sum_{l=1}^{N}\left(u_{i j}\right)^{m}\left(u_{i l}\right)^{m} D_{j l},
$$

em que $D_{j l}=\left(\mathbf{x}_{j}-\mathbf{x}_{l}\right)^{T} \mathbf{A}\left(\mathbf{x}_{j}-\mathbf{x}_{l}\right)$ é qualquer quadrado de distância induzida por produto interno entre o objeto $\mathbf{x}_{j}$ e o objeto $\mathbf{x}_{l}^{5}$ (e.g. distância Euclideana quadrada quando $\mathbf{A}$ é a matriz identidade). Dessa forma, os objetos que estão próximos (i.e. valores baixos de $D_{j l}$ ) tendem a apresentar valores altos de $u_{i j}$ e $u_{i l}$ e, consequentemente, tendem a estarem no mesmo grupo.

Considerando $D_{j l}$ fixo, as condições necessárias para minimizar (2.22) são dadas por $(1 \leq i \leq k, 1 \leq j \leq N)$ :

$$
u_{i j}=\frac{\left(\sum_{l=1}^{N}\left(u_{i l}\right)^{m} D_{j l}\right)^{1 /(1-m)}}{\sum_{c=1}^{k}\left(\sum_{l=1}^{N}\left(u_{c l}\right)^{m} D_{j l}\right)^{1 /(1-m)}} .
$$

O autor sugere então utilizar uma técnica similar à usada pelo algoritmo FCM, i.e., uma otimização alternada entre os valores de $u_{i j}$. Os valores podem ser atualizados de duas formas: online e em batch. No modo online, as pertinências são atualizadas e já substituídas na matriz de partição. No modo em batch, toda a atualização é feita em uma matriz de partição temporária $\left(\mathbf{U}^{\prime}\right)$ e somente depois de todos os elementos serem atualizados é que a matriz de partição original é substituída pela matriz temporária $\left(\mathbf{U} \leftarrow \mathbf{U}^{\prime}\right)$. Entretanto, o autor alega que a atualização em batch é muito instável e a partição final pode apresentar várias pertinências rígidas (próximas de 1) em alguns objetos aleatórios. Dessa forma, somente os passos do modo online são ilustrados no Algoritmo 7.

O Passo 3 do Algoritmo 7 embaralha a ordem dos objetos na matriz de dados para

\footnotetext{
${ }^{5}$ Mais precisamente, o autor afirma que $D_{j l}$ também pode considerar o tamanho e o formato dos grupos.
} 


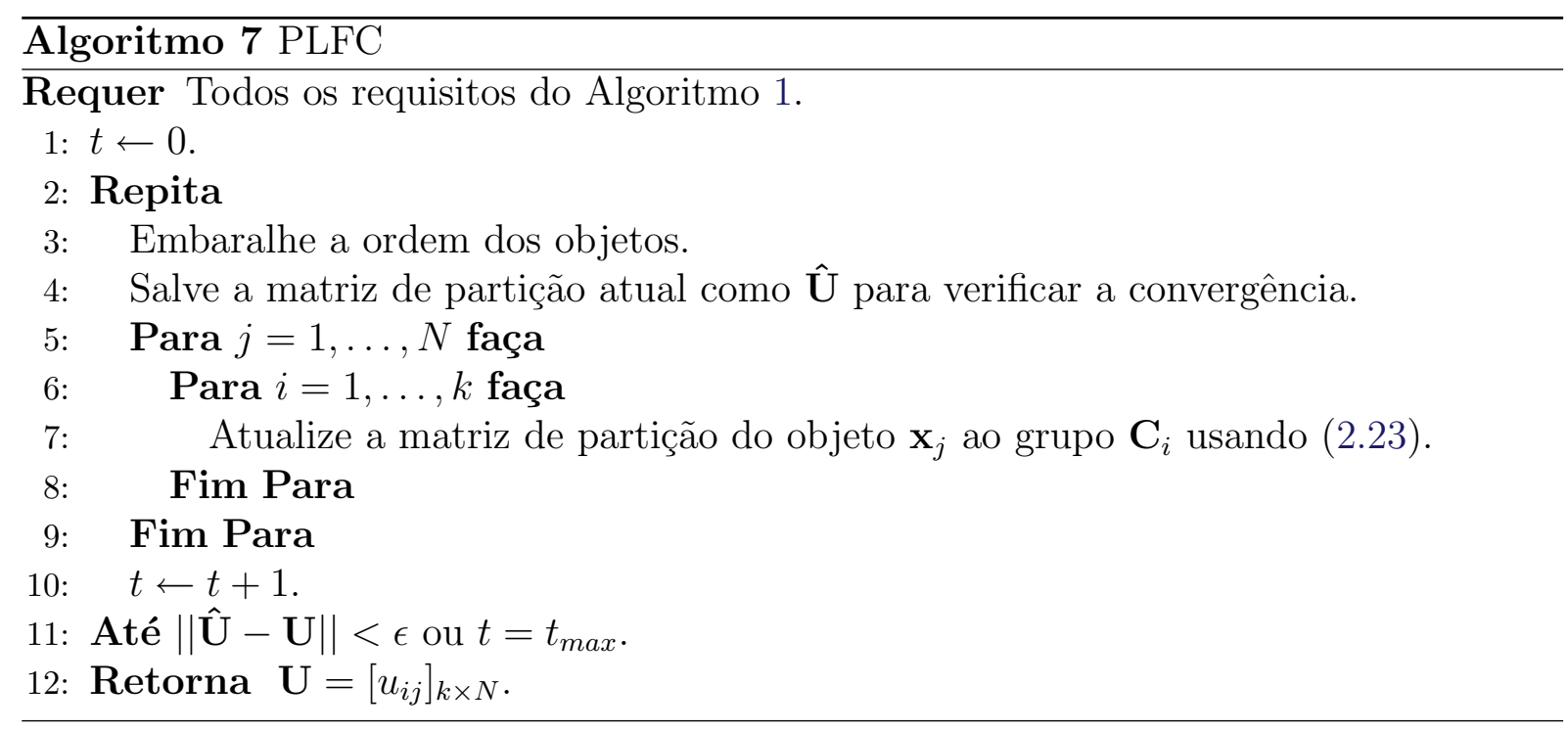

evitar efeitos que poderiam ser obtidos devido à ordem inicial dos dados (Borgelt, 2007). A atualização das pertinências deve ser executada objeto a objeto (para cada cada objeto a pertinência para todos os grupos são atualizadas) e, por este motivo, os passos 5 a 9 do Algoritmo 7 explicitam a ordem dos laços a ser executada.

\section{Análise de Complexidade - Tempo}

Embaralhar os objetos requer $O(N)$ operações. O cálculo das distâncias entre todos pares os objetos requer $O\left(n^{2} N^{2}\right)$. A atualização da matriz de partição nos passos 5 a 9 do Algoritmo 7 requer $O\left(k^{2} N^{2}\right)$. Portanto, a complexidade de tempo do algoritmo de agrupamento PLFC é $O\left(t\left(N+n^{2} N^{2}+k^{2} N^{2}\right)\right) \rightarrow O\left(t\left(n^{2} N^{2}+k^{2} N^{2}\right)\right)$.

\section{Análise de Complexidade - Espaço}

A base de dados $\mathbf{X}$ requer $O(N n)$ unidades de espaço. A saída do algoritmo requer $O(k N)$ para a matriz de partição. As distâncias são calculadas e armazenadas em uma tabela à parte, o que requer $O\left(N^{2}\right)$ unidades de espaço. Portanto, dado que $N>k$, a complexidade de espaço do algoritmo de agrupamento PLFC é $O\left(N n+k N+N^{2}\right) \rightarrow$ $O\left(N n+N^{2}\right)$.

\subsubsection{Algoritmos que Buscam por Contornos}

Os algoritmos apresentados anteriormente foram desenvolvidos para encontrar grupos globulares (hiperesféricos ou hiperelípticos) e grupos com formatos de retas, planos ou hiperplanos. Entretanto, um tipo de grupo de bastante interesse, principalmente em processamento de imagens, consiste em contornos de objetos, "cascas" ou extremidades (shells — em inglês). Nesta seção são apresentados alguns desses algoritmos. 


\subsubsection{FCS: Fuzzy c-Shells}

O algoritmo FCS (Fuzzy c-Shells — em inglês) (Dave, 1992) procura por contornos de círculos e podem ser utilizados para detecção de bordas circulares em imagens (Hoppner et al., 1999). Cada grupo é caracterizado pelo seu centro $\mathbf{v}_{i}$ e um raio $r_{i}$. Dessa forma, a distância de um objeto $\mathbf{x}_{j}$ ao $i$-ésimo grupo é dada por $(1 \leq i \leq k, 1 \leq j \leq N)$ :

$$
D_{i j}=\left|d_{i j}-r_{i}\right|^{2}
$$

em que |.| representa o valor absoluto e $d_{i j}=\left\|\mathbf{x}_{j}-\mathbf{v}_{i}\right\|$ é a distância Euclideana entre o objeto $\mathbf{x}_{j}$ e o protótipo $\mathbf{v}_{i}{ }^{6}$.

Minimizar (2.6) em relação aos protótipos $\mathbf{v}_{i}$ e aos raios $r_{i}$ com as distâncias definidas em (2.24) leva às seguintes condições necessárias (Dave, 1992) $(1 \leq i \leq k, 1 \leq j \leq N)$ :

$$
\begin{gathered}
\sum_{j=1}^{N}\left(u_{i j}\right)^{m} \frac{\left|d_{i j}-r_{i}\right|}{d_{i j}}\left(\mathbf{x}_{j}-\mathbf{v}_{i}\right)=\mathbf{0} \\
\sum_{j=1}^{N}\left(u_{i j}\right)^{m}\left|d_{i j}-r_{i}\right|=\mathbf{0}
\end{gathered}
$$

Essas condições necessárias formam um sistema de equações não lineares em $\mathbf{v}_{i}$ e $r_{i}$. Para solucionar esse problema Dave (1992) sugere que o método de Newton seja utilizado para encontrar os valores de $\mathbf{v}_{i}$ e $r_{i}$ a cada iteração do algoritmo.

O procedimento iterativo do algoritmo FCS é descrito no Algoritmo 8.

\section{Análise de Complexidade - Tempo}

O cálculo dos protótipos e raios dos grupos em (2.25) e (2.26) requer $O\left(k N n t_{N}\right)$ operações ( $t_{N}$ é o número de iterações do método de Newton). O cálculo das distâncias em (2.24) requer $O(k N n)$. A atualização das pertinências em (2.8) requer $O(k N)$ operações, usando o mesmo procedimento adotado por Kolen \& Hutcheson (2002). Portanto, a complexidade de tempo do algoritmo de agrupamento FCS é $O\left(t\left(k N n t_{N}+k N n+k N\right)\right) \rightarrow$ $O\left(t k N n t_{N}\right)$.

\section{Análise de Complexidade - Espaço}

A base de dados $\mathbf{X}$ requer $O(N n)$ unidades de espaço. As saídas do algoritmo requerem $O(k N)$ para a matriz de partição, $O(k n)$ para os protótipos dos grupos e $O(k)$ para os raios dos grupos. As distâncias são calculadas e armazenadas em uma tabela à parte, o

\footnotetext{
${ }^{6}$ Note que a distância utilizada pelo algoritmo FCS definida em (2.24) é diferente da distância utilizada pelo algoritmo EFCM em (2.20). No algoritmo FCS, a definição de distância mede o quão próximo um objeto está do perímetro da circunferência de centro $\mathbf{v}_{i}$ e raio $r_{i}$, enquanto que no algoritmo EFCM, a definição de distância mede o quão próximo um objeto está do protótipo $\mathbf{v}_{i}$ considerando que tal protótipo é uma hiperesfera de raio $r_{i}$.
} 


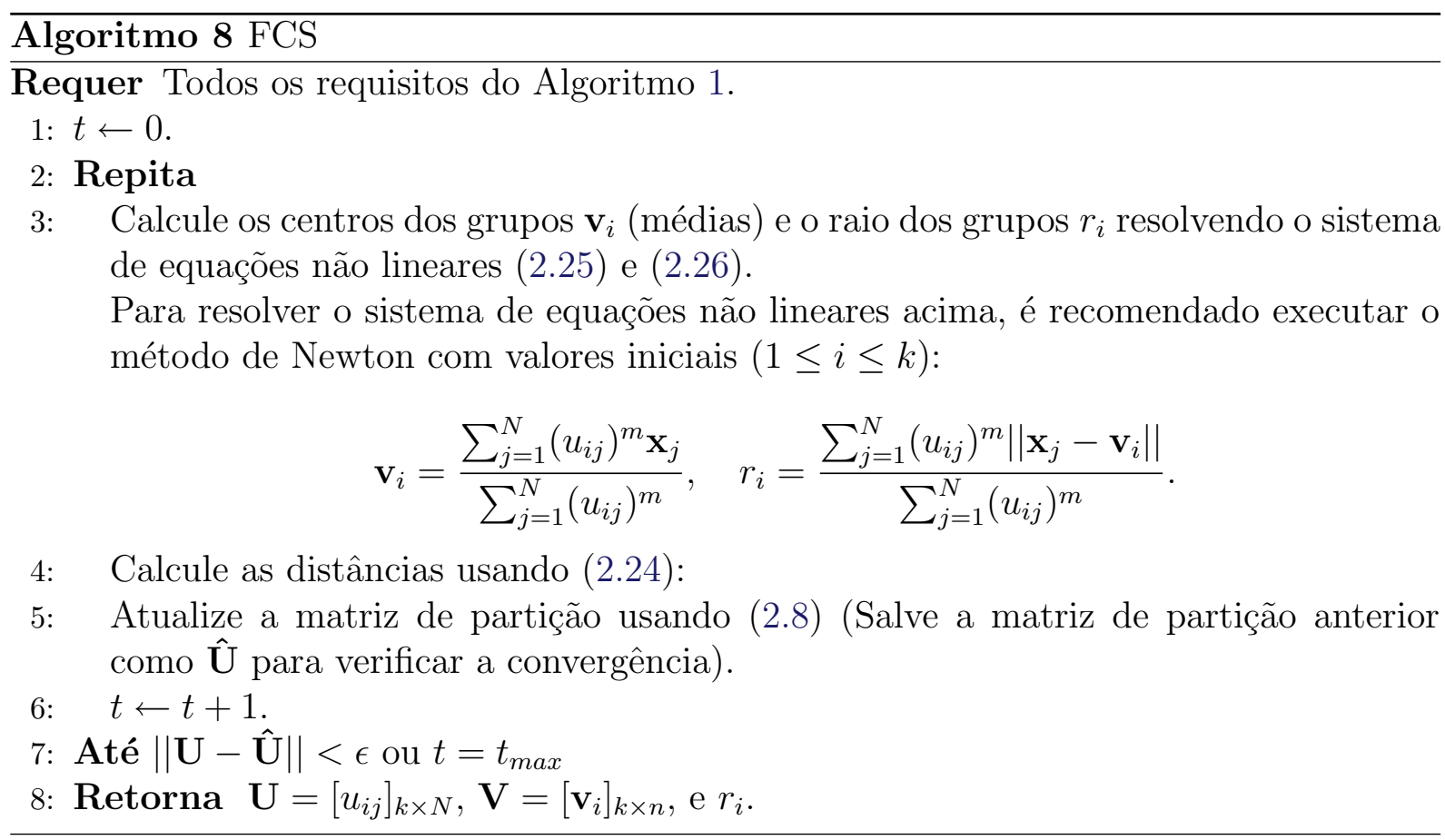

que requer $O(k N)$ unidades de espaço. Portanto, a complexidade de espaço do algoritmo de agrupamento FCS é $O(N n+k N+k n+k+k N) \rightarrow O(N n+k N)$.

\subsubsection{FCSS: Fuzzy c-Spherical Shells}

A principal desvantagem do algoritmo FCS é o custo computacional de resolver um sistema de equações não lineares a cada iteração. Para contornar esse problema, Krishnapuram et al. (1992) propuseram minimizar (2.6) usando a distância dada por $(1 \leq i \leq k ; 1 \leq j \leq N)$ :

$$
D_{i j}=\left(\left\|\mathbf{x}_{j}-\mathbf{v}_{i}\right\|^{2}-r_{i}^{2}\right)^{2}
$$

e reescreveram (2.27) como:

$$
D_{i j}=\mathbf{p}_{i}^{T} \mathbf{M}_{j} \mathbf{p}_{i}+\mathbf{c}_{j}^{T} \mathbf{p}_{i}+b_{j}
$$

em que

$$
\begin{array}{r}
b_{j}=\left(\mathbf{x}_{j}^{T} \mathbf{x}_{j}\right)^{2}, \\
\mathbf{y}_{j}=\left(\begin{array}{c}
\mathbf{x}_{j} \\
1
\end{array}\right), \\
\mathbf{c}_{j}=2\left(\mathbf{x}_{j}^{T} \mathbf{x}_{j}\right) \mathbf{y}_{j}, \\
\mathbf{M}_{j}=\mathbf{y}_{j} \mathbf{y}_{j}^{T}, \\
\mathbf{p}_{i}=\left(\begin{array}{c}
-2 \mathbf{v}_{i} \\
\mathbf{v}_{i}^{T} \mathbf{v}_{i}-r_{i}^{2}
\end{array}\right)
\end{array}
$$


Assumindo que os vetores $\mathbf{p}_{i}$ são independentes, minimizar (2.6) em relação a $\mathbf{p}_{i}$ com as distâncias dadas em (2.28) leva a (Krishnapuram et al., 1992) $(1 \leq i \leq k)$ :

$$
\mathbf{p}_{i}=-\frac{1}{2}\left(\mathbf{H}_{i}\right)^{-1} \mathbf{w}_{i}
$$

em que

$$
\begin{array}{r}
\mathbf{H}_{i}=\sum_{j=1}^{N}\left(u_{i j}\right)^{m} \mathbf{M}_{j}, \\
\mathbf{w}_{i}=\sum_{j=1}^{N}\left(u_{i j}\right)^{m} \mathbf{c}_{j} .
\end{array}
$$

O procedimento iterativo do algoritmo FCSS é descrito no Algoritmo 9.

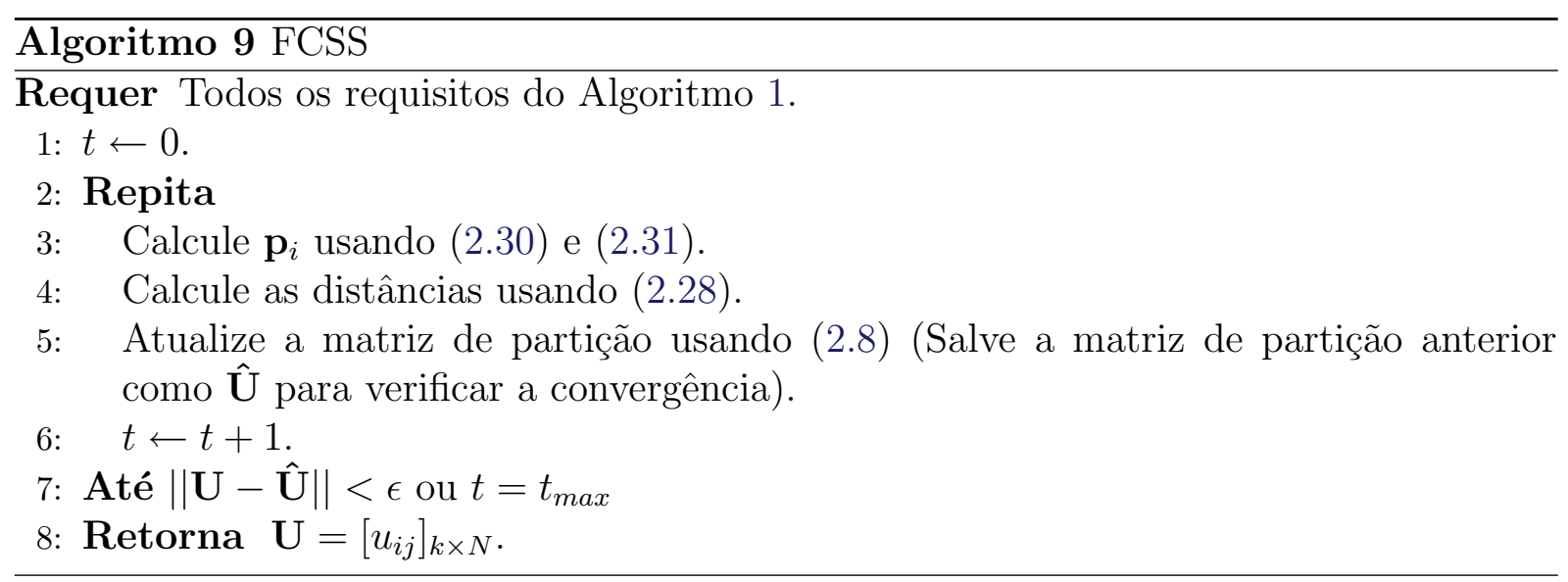

\section{Análise de Complexidade - Tempo}

O cálculo de $\mathbf{H}_{i}$ e $\mathbf{w}_{i}$ em (2.31) requer $O\left(k N n^{2}+k N n\right)$ operações. O cálculo de $\mathbf{p}_{i}$ em (2.30) requer $O\left(k n^{3}+k n^{2}\right)$. O cálculo das distâncias em (2.28) requer $O\left(k N n^{2}\right)$. A atualização das pertinências em (2.8) requer $O(k N)$ operações, usando o mesmo procedimento adotado por Kolen \& Hutcheson (2002). Portanto, a complexidade de tempo do algoritmo de agrupamento FCSS é $O\left(t\left(k N n^{2}+k N n+k n^{3}+k n^{2}+k N\right)\right) \rightarrow O\left(t\left(k N n^{2}+k n^{3}\right)\right)$.

\section{Análise de Complexidade - Espaço}

A base de dados $\mathbf{X}$ requer $O(N n)$ unidades de espaço. A matriz de partição requer $O(k N) . \mathbf{H}_{i}$ e $\mathbf{w}_{i}$ em (2.31) requerem $O\left(k n^{2}\right)$. $\mathbf{p}_{i}$ em (2.30) requer $O(k n)$. As distâncias são calculadas e armazenadas em uma tabela à parte, o que requer $O(k N)$ unidades de espaço. Portanto, a complexidade de espaço do algoritmo de agrupamento FCSS é $O\left(N n+k N+k n+k n^{2}+k N\right) \rightarrow O\left(N n+k N+k n^{2}\right)$. 


\subsubsection{Algoritmos Possibilísticos}

Os algoritmos de agrupamento derivados do FCM (incluindo todos os algoritmos descritos nas seções anteriores) utilizam a restrição probabilística apresentada em (2.5) para a matriz de partição, i.e., $\mathbf{U} \in \mathbf{M}_{f p}$. Entretanto, os graus de pertinência gerados por tais algoritmos podem não corresponder ao que é chamado de tipicidade. Essas situações ocorrem quando a pertinência de um objeto a todos os grupos não necessariamente deve somar 1 e, portanto, a matriz de partição correspondente não necessariamente satisfaz a restrição probabilística. Nesta seção são discutidos alguns algoritmos de agrupamento possibilísticos, i.e., algoritmos que utilizam a definição $\mathbf{U} \in \mathbf{M}_{f}$.

\subsubsection{PCM: Possibilistic c-Means}

Os algoritmos apresentados anteriormente trabalham com partições em $\mathbf{M}_{f p}$. Contudo, a restrição $\sum_{i=1}^{k} u_{i j}=1$ pode levar a resultados não desejados. Tome como exemplo dois grupos $\mathbf{C}_{1}$ e $\mathbf{C}_{2}$ com algum grau de sobreposição ilustrado na Figura 2.1. O grau de pertinência do objeto $\mathbf{x}_{1}$ é igual a 0,5 para ambos grupos (i.e., $u_{11}=u_{21}=0.5$ ) pois as distâncias entre $\mathbf{x}_{1}$ e os protótipos dos grupos $\mathbf{v}_{1}$ e $\mathbf{v}_{2}\left(D_{11}\right.$ e $\left.D_{21}\right)$ são as mesmas $\left(D_{11}=\right.$ $D_{21}$ ). Tal comportamento é esperado dado que $\mathbf{x}_{1}$ está em uma área de sobreposição dos grupos. Note, contudo, que o grau de pertinência do objeto outlier ${ }^{7} \mathbf{x}_{2}$ também é igual a 0,5 para ambos grupos $\left(u_{11}=u_{21}=0.5\right)$. Isso ocorre pois, embora o objeto seja um outlier, ele apresenta a mesma distância para os protótipos de ambos grupos (i.e., $\left.D_{12}=D_{22}\right)$ e, portanto, o cálculo das pertinências em (2.8) resulta em graus de pertinência iguais para ambos grupos.

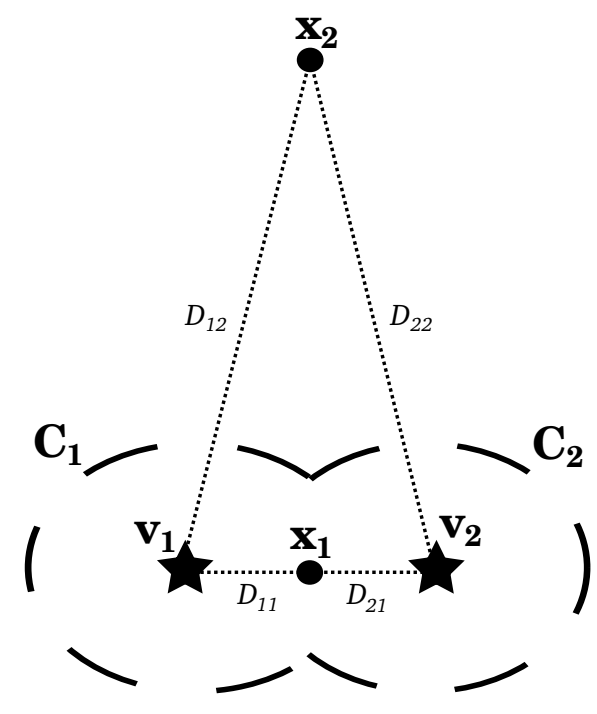

Figura 2.1: Exemplo de grau de pertinência de dois objetos (representados por pontos) a dois grupos (cujos protótipos estão representados por estrelas).

Tentando evitar esse problema, o algoritmo de agrupamento PCM (Possibilistic c-

\footnotetext{
${ }^{7}$ Um outlier é um objeto que está isolado, suficientemente longe dos demais objetos (Jain \& Dubes, 1988).
} 
Means - em inglês) (Krishnapuram \& Keller, 1993) trabalha com partições em $\mathbf{M}_{f}$. Como agora $u_{i j}$ (denominados tipicidades - typicalities em inglês) podem ser tão pequenos quanto possíveis (próximos a 0), a função objetivo em (2.6) deve ser modificada para previnir que $u_{i j} \rightarrow 0$ para todo $i, j$. Assim, o algoritmo PCM busca minimizar a seguinte função objetivo:

$$
J=\sum_{i=1}^{k} \sum_{j=1}^{N}\left(u_{i j}\right)^{m} D_{i j}+\sum_{i=1}^{k} \eta_{i} \sum_{j=1}^{N}\left(1-u_{i j}\right)^{m}
$$

em que $D_{i j}$ é a distância definida em (2.9) e $\eta_{i}>0$ é um parâmetro possibilístico definido pelo usuário. O primeiro termo de (2.32) é idêntico a (2.6). O segundo termo faz com que as tipicidades $u_{i j}$ sejam as maiores possíveis, evitando que a solução trivial $(\mathbf{U} \approx \mathbf{0})$ seja encontrada ${ }^{8}$.

Novamente o método mais popular para solucionar (2.32) é aplicar a interação de ponto fixo (Picard iteration - em inglês) com as condições de primeira ordem para pontos estacionários de (2.32). Desse modo, fixando $D_{i j}$, encontra-se que uma condição necessária $\mathrm{e}^{9}$ :

$$
u_{i j}=\left[1+\left(\frac{D_{i j}}{\eta_{i}}\right)^{1 /(m-1)}\right]^{-1}, 1 \leq i \leq k, \quad 1 \leq j \leq N
$$

Fixando $\mathbf{v}_{i}$, encontra-se que outra condição necessária para minimizar (2.32) é dada por (2.10).

Note que $\eta_{i}$ é um parâmetro muito importante para o algoritmo e deve ser pré-definido pelo usuário. Quando $\eta_{i}$ se aproxima de 0 , a solução tende a ser trivial $(\mathbf{U} \approx 0)$. Se $\eta_{i}$ é grande, $u_{i j}$ será tão grande quanto possível. Em outras palavras, $\eta_{i}$ determina o quão o segundo termo em (2.32) é importante em relação ao primeiro. Para que ambos termos sejam ponderados de maneira igual $\eta_{i}$ deve ser da ordem de $D_{i j}$. Na prática, Krishnapuram \& Keller (1993) encontraram a seguinte definição, que funciona bem para uma dada partição inicial ${ }^{10}$ :

$$
\eta_{i}=\frac{\sum_{j=1}^{N}\left(u_{i j}\right)^{m} D_{i j}}{\sum_{j=1}^{N}\left(u_{i j}\right)^{m}}, 1 \leq i \leq k
$$

O procedimento completo do algoritmo PCM é descrito no Algoritmo 10.

\footnotetext{
${ }^{8}$ Lembre-se que $\mathbf{U} \in \mathbf{M}_{f}$.

${ }^{9}$ Note que as colunas de $\mathbf{U}$ são independentes e, portanto, é possível decompor a função objetivo em $k$ componentes individuais. Assim, encontra-se que a atualização da pertinência de um objeto a um grupo não depende mais das distâncias desse objeto aos outros grupos como ocorre em (2.8).

${ }^{10}$ Por exemplo, uma partição obtida por uma execução prévia do algoritmo FCM.
} 


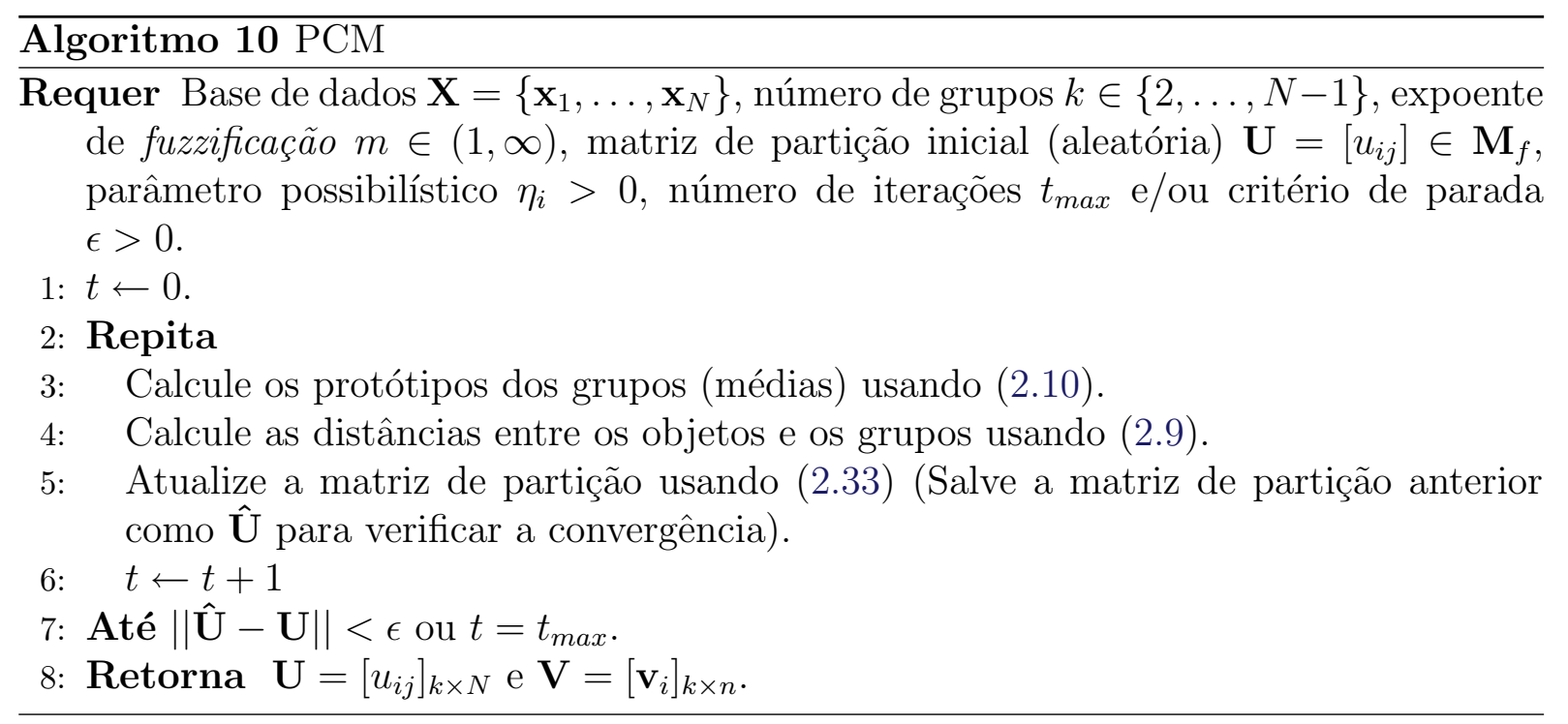

\section{Análise de Complexidade - Tempo}

O cálculo dos protótipos em (2.10) requer $O(k N n)$ operações. O cálculo das distâncias em (2.9) requer $O\left(k N n^{2}\right)$ operações. A atualização da matriz de partição possibilística em (2.33) requer $O(k N)$ operações. Portanto, a complexidade de tempo do algoritmo de agrupamento PCM é $O\left(t\left(k N n+k N n^{2}+k N\right)\right) \rightarrow O\left(t k N n^{2}\right)$.

\section{Análise de Complexidade - Espaço}

A base de dados $\mathbf{X}$ requer $O(N n)$ unidades de espaço. As saídas do algoritmo requerem $O(k N)$ para a matriz de partição e $O(k n)$ para os protótipos dos grupos. As distâncias são calculadas e armazenadas em uma tabela à parte, o que requer $O(k N)$ unidades de espaço. Para o cálculo das distâncias usando (2.9) é necessário armazenar a matriz de norma induzida A, que requer $O\left(n^{2}\right)$. Portanto, a complexidade de espaço do algoritmo de agrupamento PCM é $O\left(N n+k N+k n+k N+n^{2}\right) \rightarrow O\left(N n+k N+k n+n^{2}\right) \rightarrow$ $O\left(N n+k N+n^{2}\right)$.

\subsubsection{PGK: Possibilistic Gustafson-Kessel}

O algoritmo de agrupamento PGK (Possibilistic Gustafson-Kessel — em inglês) (Krishnapuram \& Keller, 1993) é uma versão possibilística do algoritmo GK (Seção 2.2.2.1). Esse algoritmo usa as distâncias definidas em (2.11) para minimizar (2.32). Nesse caso, fixando $D_{i j}$ as condições necessárias para minimizar (2.32) também levam a (2.33). Fixando $u_{i j}$ e $\mathbf{A}_{i}$, as condições necessárias levam a (2.10). Finalmente, fixando $u_{i j}$ e $v_{i j}$, e restringindo $\mathbf{A}_{i}$ de tal forma que $\operatorname{det}\left(\mathbf{A}_{i}\right)=\rho_{i}$, as condições necessárias levam a (2.12) e (2.13). Logo, o procedimento completo é descrito no Algoritmo 11. 


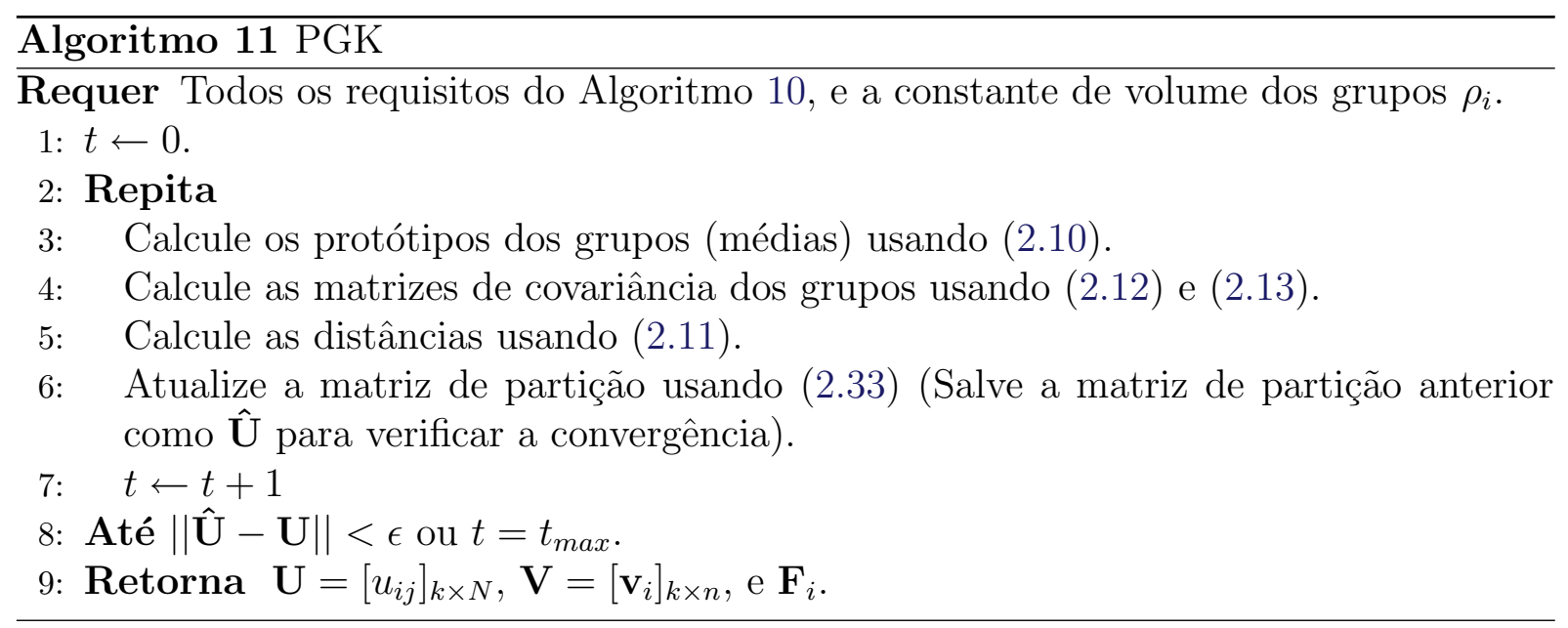

\section{Análise de Complexidade - Tempo}

O cálculo dos protótipos em (2.10) requer $O(k N n)$ operações. O cálculo de $\mathbf{A}_{i}$ em (2.12) requer $O\left(k n^{3}\right)$ operações e o cálculo das matrizes de covariância em (2.13) requer $O\left(k N n^{2}\right)$ operações. O cálculo das distâncias em (2.11) requer $O\left(k N n^{2}\right)$ operações. A atualização da matriz de partição em (2.33) requer $O(k N)$. Portanto, a complexidade de tempo do algoritmo de agrupamento PGK é $O\left(t\left(k N n+k n^{3}+k N n^{2}+k N n^{2}+k N\right)\right) \rightarrow$ $O\left(t k n^{3}+t k n^{2} N\right)$.

\section{Análise de Complexidade - Espaço}

A base de dados $\mathbf{X}$ requer $O(N n)$ unidades de espaço. As saídas do algoritmo requerem $O(k N)$ para a matriz de partição, $O(k n)$ para os protótipos dos grupos, e $O\left(k n^{2}\right)$ para as matrizes de covariância. As distâncias são calculadas e armazenadas em uma tabela à parte, o que requer $O(k N)$ unidades de espaço. Para calcular as distâncias em (2.11) é necessário armazenar $\mathbf{A}_{i}$ em (2.12) e as matrizes de covariância em (2.13), o que requer $O\left(k n^{2}\right)$. Portanto, a complexidade de espaço do algoritmo de agrupamento PGK é $O(N n+$ $\left.k N+k n+k n^{2}+k N+k n^{2}\right) \rightarrow O\left(N n+k N+k n^{2}\right)$.

\subsubsection{FPCM: Fuzzy-Possibilistic c-Means}

Devido à independência entre os grupos existente nos algoritmos derivados do PCM, é conhecido que tais algoritmos podem levar a uma solução em que os protótipos estão todos na mesma posição (ou muito próximos) (Barni et al., 1996; Timm et al., 2004). Para contornar esse problema, o algoritmo FPCM (Fuzzy-Possibilistic c-Means - em inglês) (Pal et al., 1997) busca encontrar simultaneamente pertinências $\left(u_{i j}\right)$ e tipicidades $\left(p_{i j}\right)$ entre os objetos e os grupos (i.e. uma matriz $\mathbf{U}=\left[u_{i j}\right] \in \mathbf{M}_{f p}$ e uma matriz $\left.\mathbf{P}=\left[p_{i j}\right] \in \mathbf{M}_{f}\right)$. 
A função objetivo a ser minimizada é dada por:

$$
J=\sum_{i=1}^{k} \sum_{j=1}^{N}\left(\left(u_{i j}\right)^{m}+\left(p_{i j}\right)^{\gamma}\right) D_{i j},
$$

em que $D_{i j}$ é definido por (2.9), $m, \gamma>0$ são expoentes de fuzzificação, $\mathbf{U}=\left[u_{i j}\right] \in \mathbf{M}_{f p}$ é a matriz de partição probabilística e $\mathbf{P}=\left[p_{i j}\right] \in \mathbf{M}_{f}$ é a matriz de partição possibilística. Diferentemente de uma pertinência probabilística $u_{i j}$, uma tipicidade $p_{i j}$ não depende das tipicidades atribuídas aos outros grupos $\left(p_{c j}, c \neq i\right)$. Dado que $\mathbf{P}=\left[p_{i j}\right] \in \mathbf{M}_{f}, p_{i j}$ pode ser tão pequena quanto desejado para minimizar (2.35). Para evitar esse comportamento, Pal et al. (1997) restringiram as tipicidades $\operatorname{por}^{11}(1 \leq i \leq k)$ :

$$
\sum_{j=1}^{N} p_{i j}=1
$$

Novamente, o método mais popular para solucionar (2.35) é aplicar a interação de ponto fixo com as condições de primeira ordem para pontos estacionários de (2.35). Fixando $D_{i j}$ e $p_{i j}$, as condições necessárias para minimizar (2.35) levam a (2.8), enquanto que ao fixar $D_{i j}$ e $u_{i j}$ as condições necessárias levam a $(1 \leq i \leq k, 1 \leq j \leq N)$ :

$$
p_{i j}=\left(\sum_{l=1}^{N}\left(\frac{D_{i j}}{D_{i l}}\right)^{1 /(\gamma-1)}\right)^{-1} .
$$

Finalmente, fixando $u_{i j}$ e $p_{i j}$ as condições necessárias levam a $(1 \leq i \leq k)$ :

$$
\mathbf{v}_{i}=\frac{\sum_{j=1}^{N}\left(\left(u_{i j}\right)^{m}+\left(p_{i j}\right)^{\gamma}\right) \mathbf{x}_{j}}{\sum_{j=1}^{N}\left(\left(u_{i j}\right)^{m}+\left(p_{i j}\right)^{\gamma}\right)} .
$$

O procedimento completo é então descrito no Algoritmo 12.

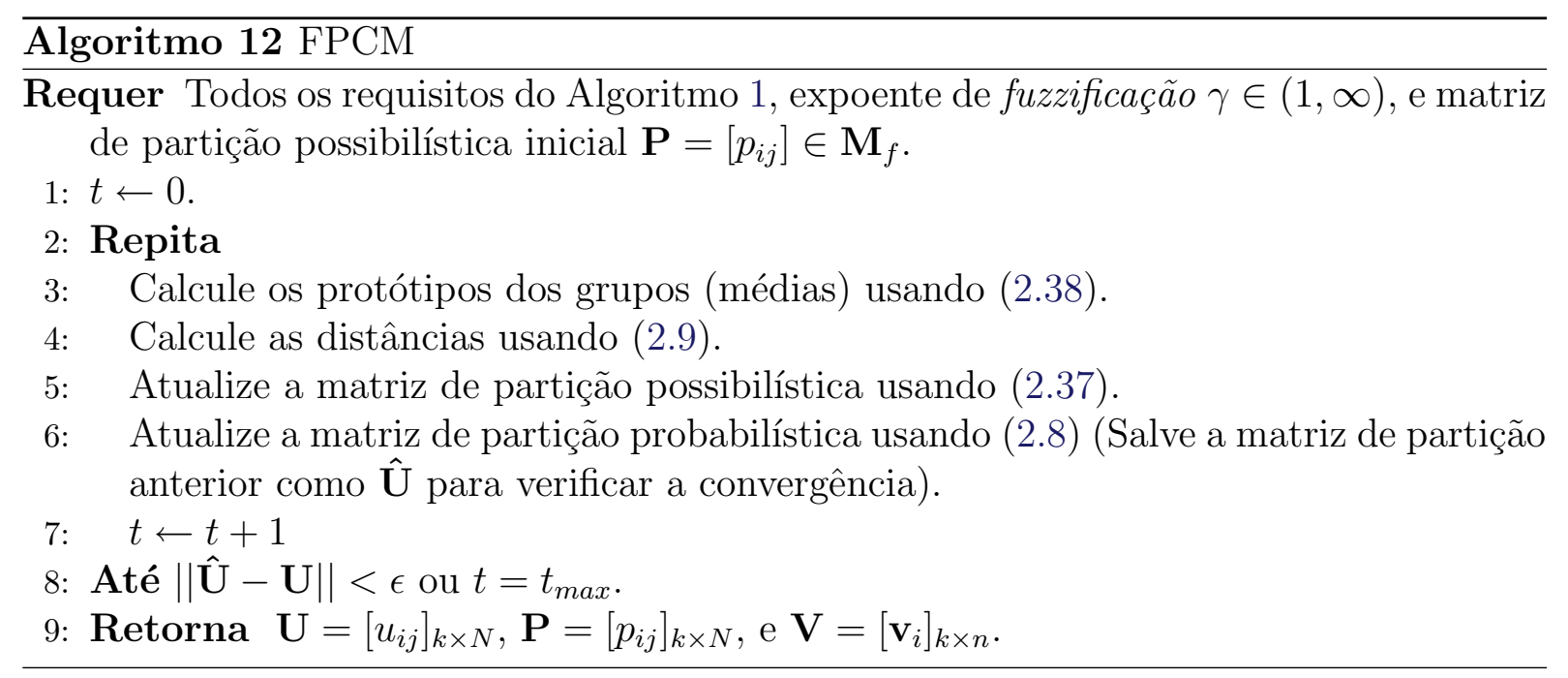

\footnotetext{
${ }^{11}$ Note que, pelo fato de $\mathbf{U} \in \mathbf{M}_{f p}$, tal matriz já é restrita por (2.5).
} 
O cálculo dos protótipos em (2.38) requer $O(k N n)$ operações. O cálculo das distâncias em (2.9) requer $O\left(k N n^{2}\right)$ operações. A atualização das pertinências em (2.8) requer $O(k N)$ usando o mesmo procedimento adotado por Kolen \& Hutcheson (2002). A atualização das pertinências em (2.37) requer $O\left(k N^{2}\right)$, usando o método mais simples. Entretanto, um método similar ao adotado por Kolen \& Hutcheson (2002) pode ser aplicado para reduzir a complexidade computacional. Note que

$$
\varphi_{i}=\sum_{l=1}^{N}\left(\frac{1}{D_{i l}}\right)^{1 /(\gamma-1)}, 1 \leq i \leq k
$$

pode ser calculado para cada grupo antes do cálculo de $p_{i j}$ em (2.37) para todo objeto $j$. Assim, (2.37) se reduz a $(1 \leq i \leq k, 1 \leq j \leq N)$

$$
p_{i j}=\left(\left(D_{i j}\right)^{1 /(\gamma-1)} \cdot \varphi_{i}\right)^{-1}
$$

que requer apenas $O(k N)$ operações para a atualização da matriz de partição possibilística. Portanto, a complexidade de tempo do algoritmo de agrupamento FPCM pode ser $O\left(t\left(k N n+k N n^{2}+k N+k N+k N\right)\right) \rightarrow O\left(t k N n^{2}\right)$ com o método acima. Até onde nós sabemos, o uso de um método similar para reduzir a complexidade do algoritmo FPCM não foi reportado anteriormente na literatura e pode ser considerado uma contribuição marginal da presente dissertação.

\section{Análise de Complexidade - Espaço}

A base de dados $\mathbf{X}$ requer $O(N n)$ unidades de espaço. As saídas do algoritmo requerem $O(k N)$ para as matrizes de partição e $O(k n)$ para os protótipos dos grupos. As distâncias são calculadas e armazenadas em uma tabela à parte, o que requer $O(k N)$ unidades de espaço. Para o cálculo das distâncias usando (2.9) é necessário armazenar a matriz A, que requer $O\left(n^{2}\right)$. Portanto, a complexidade de espaço do algoritmo de agrupamento FPCM é $O\left(N n+k N+k n+k N+n^{2}\right) \rightarrow O\left(N n+k N+k n+n^{2}\right) \rightarrow O\left(N n+k N+n^{2}\right)$.

\subsubsection{PFCM: Possibilistic-Fuzzy c-Means}

A restrição (2.36) imposta pelo algoritmo FPCM (Seção 2.2.5.3) sobre os termos da matriz possibilística $\mathbf{P}$ faz com que, para bases de dados em que $N$ é grande, os valores de tipicidade $p_{i j}$ sejam muito pequenos. Para contornar esse problema, o algoritmo de agrupamento PFCM (Possibilistic-Fuzzy c-Means — em inglês) (Pal et al., 2005) relaxa a restrição (2.36) para os valores de tipicidade, porém busca minimizar a seguinte função objetivo: 


$$
J=\sum_{i=1}^{k} \sum_{j=1}^{N}\left(a \cdot\left(u_{i j}\right)^{m}+b \cdot\left(p_{i j}\right)^{\gamma}\right) D_{i j}+\sum_{i=1}^{k} \eta_{i} \sum_{j=1}^{N}\left(1-p_{i j}\right)^{\gamma}
$$

em que $\mathbf{U}=\left[u_{i j}\right] \in \mathbf{M}_{f p}, \mathbf{P}=\left[p_{i j}\right] \in \mathbf{M}_{f}, a>0$ e $b>0$ definem as importâncias relativas entre pertinências (probabilísticas) e tipicidades (possibilísticas), $m>1$ e $\gamma>1$ são expoentes de fuzzificação e $\eta_{i}>0$ são constantes possibilísticas definidas pelo usuário (veja a Seção 2.2.5.1). Note que se $a=0$ então (2.40) se reduz a (2.32) (função objetivo do algoritmo PCM). Em contrapartida, se $b=0$ e $\eta_{i}=0 \forall i$, então (2.40) se reduz a (2.6) (função objetivo do algoritmo $\mathrm{FCM}$ ). De fato, se $b=0$ então $p_{i j}$ não é afetado por $D_{i j}$ (consequentemente pelos objetos $\mathbf{x}_{j}$ e centros $\mathbf{v}_{i}$ ) e, portanto, minimizar $(2.40)$ (função objetivo do algoritmo PFCM) é equivalente a minimizar (2.6) (função objetivo do algoritmo FCM).

O método para solucionar (2.40) consiste em aplicar a iteração de ponto fixo com as condições de primeira ordem para pontos estacionários de (2.40). Fixando $D_{i j}$ e $p_{i j}$ as condições necessárias para minimizar (2.40) levam a (2.8), enquanto que ao fixar $D_{i j}$ e $u_{i j}$ as condições necessárias levam a $(1 \leq i \leq k, 1 \leq j \leq N)$ :

$$
p_{i j}=\left(1+\left(\frac{b}{\eta_{i}} D_{i j}\right)^{1 /(\gamma-1)}\right)^{-1}
$$

Finalmente, fixando $u_{i j}$ e $p_{i j}$ as condições necessárias levam a $(1 \leq i \leq k)$ :

$$
\mathbf{v}_{i}=\frac{\sum_{j=1}^{N}\left(a \cdot\left(u_{i j}\right)^{m}+b \cdot\left(p_{i j}\right)^{\gamma}\right) \mathbf{x}_{j}}{\sum_{j=1}^{N}\left(a \cdot\left(u_{i j}\right)^{m}+b \cdot\left(p_{i j}\right)^{\gamma}\right)}
$$

O procedimento iterativo é então ilustrado no Algoritmo 13.

\section{Análise de Complexidade - Tempo}

O cálculo dos protótipos em (2.42) requer $O(k N n)$ operações. O cálculo das distâncias em (2.9) requer $O\left(k N n^{2}\right)$ operações. A atualização da matriz de partição em (2.8) requer $O(k N)$ usando o mesmo procedimento adotado por Kolen \& Hutcheson (2002). A atualização da matriz de partição possibilística em (2.41) requer $O(k N)$. Portanto, a complexidade de tempo do algoritmo de agrupamento PFCM é $O\left(t\left(k N n+k N n^{2}+k N+k N+k N\right)\right) \rightarrow O\left(t k N n^{2}\right)$.

\section{Análise de Complexidade - Espaço}

A base de dados requer $O(N n)$ unidades de espaço. As saídas do algoritmo requerem $O(k N)$ para as matrizes de partição e $O(k n)$ para os protótipos dos grupos. As distâncias 


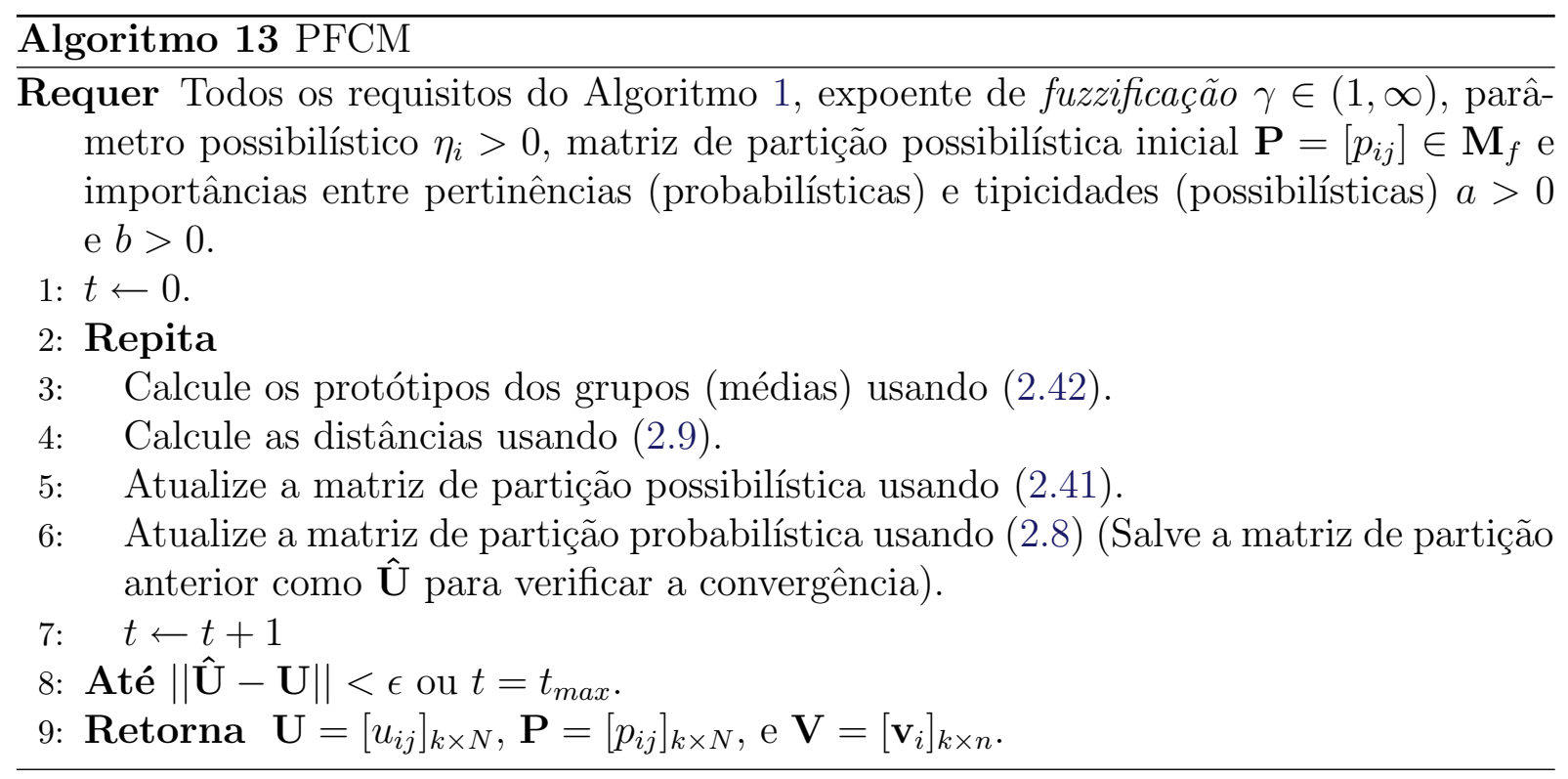

são calculadas e armazenadas em uma tabela à parte, o que requer $O(k N)$ unidades de espaço. Para o cálculo das distâncias usando (2.9) é necessário armazenar A, que requer $O\left(n^{2}\right)$. Portanto, a complexidade de espaço do algoritmo de agrupamento PFCM é $O\left(N n+k N+k n+k N+n^{2}\right) \rightarrow O\left(N n+k N+k n+n^{2}\right) \rightarrow O\left(N n+k N+n^{2}\right)$.

\subsubsection{Algoritmos Relacionais}

Todos os algoritmos descritos anteriormente necessitam da matriz de dados para o cálculo das distâncias e dos protótipos. Em outras palavras, os algoritmos apresentados até agora necessitam de todos os atributos dos objetos da base de dados. Entretanto, existem situações nas quais os atributos dos objetos não são conhecidos ou não podem ser disponibilizados por problemas de confidencialidade. Nesses casos, a única informação obtida consiste nas distâncias entre os pares de objetos da base de dados.

Formalmente, é fornecida ao algoritmo a matriz relacional dos dados $\mathbf{R}=\left[r\left(\mathbf{x}_{i}, \mathbf{x}_{j}\right)\right] \in \mathbb{R}^{N \times N}$, contendo a informação de dissimilaridade (ou similaridade) entre os pares de objetos $\mathbf{x}_{i}$ e $\mathbf{x}_{j}(i=1, \ldots, N ; j=1, \ldots, N)$. Tome como exemplo a matriz de dados $\mathbf{X}$ ilustrada na Tabela 2.5 com quatro objetos e três atributos. Considere que os atributos são confidenciais e não podem ser fornecidos para o algoritmo de agrupamento que está em operação em outra localidade. Nesse caso é possível calcular previamente as distâncias entre os pares de objetos e então fornecer para o algoritmo de agrupamento tais distâncias. Na Tabela 2.6 estão ilustradas as distâncias (Euclideanas quadradas) entre os pares de objetos da Tabela 2.5. O objetivo de um algoritmo de agrupamento relacional é, então, obter uma estrutura dos objetos analisando essa matriz relacional $\mathbf{R}$.

Nesta seção são apresentados alguns dos algoritmos de agrupamento fuzzy relacionais mais conhecidos e utilizados na literatura. 


$\mathbf{X}=$\begin{tabular}{|c|c|c|c|}
\hline & Atributo 1 & Atributo 2 & Atributo 3 \\
\hline Objeto 1 & 1 & 0 & 2 \\
\hline Objeto 2 & 3 & 1 & 0 \\
\hline Objeto 3 & 5 & 0 & 2 \\
\hline Objeto 4 & 3 & 4 & 1 \\
\hline \hline
\end{tabular}

Tabela 2.5: Matriz de dados com quatro objetos descritos por três atributos.

$\mathbf{R}=$\begin{tabular}{|l|c|c|c|c|}
\hline & Objeto 1 & Objeto 2 & Objeto 3 & Objeto 4 \\
\hline Objeto 1 & 0 & 9 & 16 & 21 \\
\hline Objeto 2 & 9 & 0 & 9 & 10 \\
\hline Objeto 3 & 16 & 9 & 0 & 21 \\
\hline Objeto 4 & 21 & 10 & 21 & 0 \\
\hline \hline
\end{tabular}

Tabela 2.6: Matriz relacional dos dados contendo as distâncias Euclideanas quadradas entre os objetos ilustrados na Tabela 2.5.

\subsubsection{FCMdd: Fuzzy c-Medoids}

O algoritmo FCMdd (Fuzzy c-Medoids — em inglês) (Krishnapuram et al., 1999, 2001) baseia-se no conceito de medóides. Um medóide é um objeto que, em tese, melhor representa o grupo. Algoritmos baseados em medóides tendem a ser menos sensíveis a outliers do que algoritmos baseados em protótipos que são pontos médios dos grupos. Adicionalmente, esses algoritmos não utilizam informações sobre os atributos dos objetos, necessitando apenas das distâncias entre eles. Em contrapartida, o custo de se obter medóides geralmente é mais alto do que se obter pontos médios.

Seja, nesse caso, $\mathbf{X}=\left\{\mathbf{x}_{1}, \ldots, \mathbf{x}_{N}\right\}$ um conjunto de $N$ objetos, em que $\mathbf{x}_{i}$ representa o $i$ ésimo objeto da base de dados. Sejam também os medóides $\mathbf{V}=\left\{\mathbf{v}_{1}, \mathbf{v}_{2}, \ldots, \mathbf{v}_{k}\right\}, \mathbf{v}_{i} \in \mathbf{X}$, um subconjunto de objetos de $\mathbf{X}$ com cardinalidade $k$, i.e., o conjunto dos medóides $\mathbf{V}$ é um subconjunto de tamanho $k$ de $\mathbf{X}$. Então, formalmente, o algoritmo FCMdd é guiado a minimizar a seguinte equação:

$$
J_{F C M d d}=\sum_{i=1}^{k} \sum_{j=1}^{N}\left(u_{i j}\right)^{m} r\left(\mathbf{x}_{j}, \mathbf{v}_{i}\right)
$$

em que $r\left(\mathbf{x}_{j}, \mathbf{v}_{i}\right)$ é a dissimilaridade entre o objeto $\mathbf{x}_{j}$ e o medóide (objeto) $\mathbf{v}_{i}$, ou seja, o elemento da matriz relacional $\mathbf{R}$ correspondente a este par de objetos. A minimização da função é feita sobre todo $\mathbf{V}$ em $\mathbf{X}$. Desta forma os medóides são selecionados de acordo com $(1 \leq i \leq k)$ :

$$
\mathbf{v}_{i}=\left\{\mathbf{x}_{l} \mid \underset{l}{\operatorname{argmin}}\left\{\sum_{j=1}^{N}\left(u_{i j}\right)^{m} r\left(\mathbf{x}_{l}, \mathbf{x}_{j}\right)\right\}\right\} .
$$

De maneira similar ao algoritmo FCM, considerando $r\left(\mathbf{x}_{j}, \mathbf{v}_{i}\right)$ constantes, minimizar 
(2.43) em relação a $u_{i j}$ leva a $(1 \leq i \leq k, 1 \leq j \leq N)$ :

$$
u_{i j}=\left(\sum_{c=1}^{k}\left(\frac{r\left(\mathbf{x}_{j}, \mathbf{v}_{i}\right)}{r\left(\mathbf{x}_{j}, \mathbf{v}_{c}\right)}\right)^{1 /(m-1)}\right)^{-1} .
$$

O procedimento iterativo do algoritmo FCMdd é descrito no Algoritmo 14.

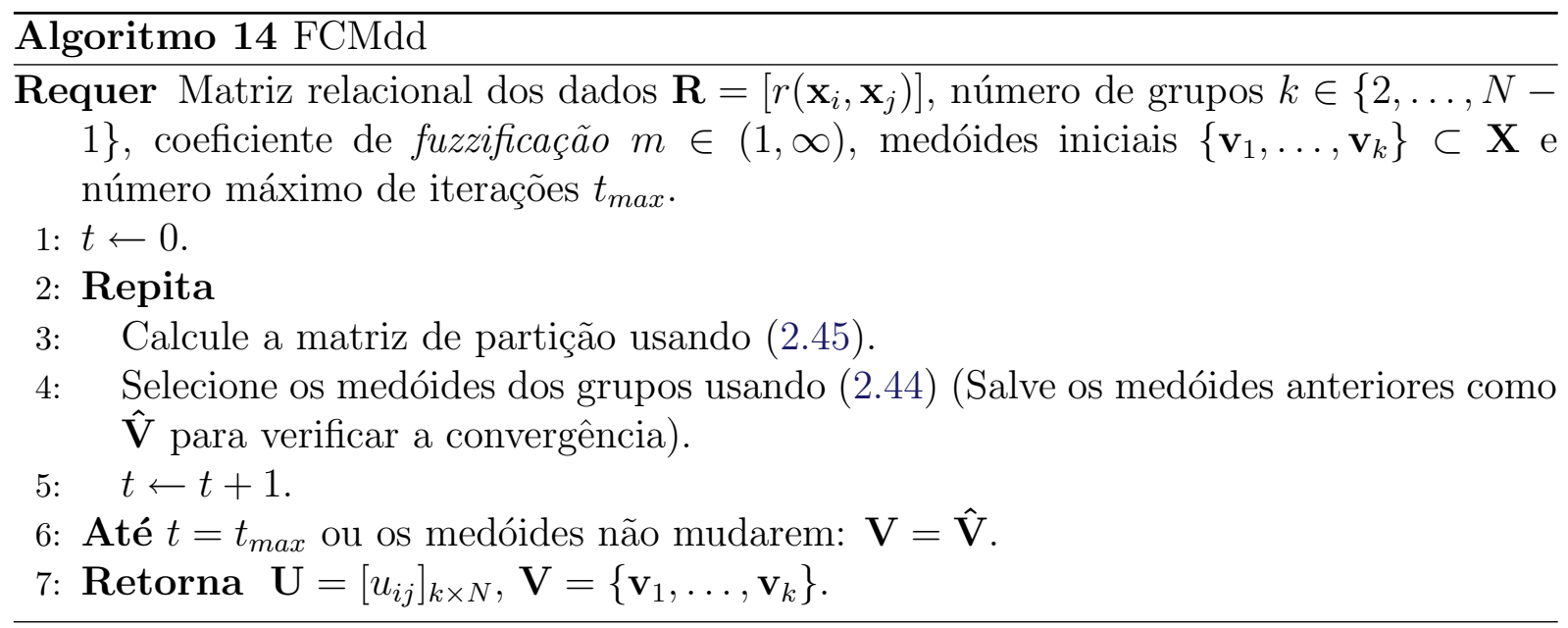

\section{Análise de Complexidade - Tempo}

A atualização das pertinências em (2.45) requer $O(k N)$ operações, usando o mesmo procedimento adotado por Kolen \& Hutcheson (2002). A seleção dos medóides usando (2.44) requer $O\left(k N^{2}\right)$. Portanto, a complexidade de tempo do algoritmo de agrupamento FCMdd é $O\left(t\left(k N+k N^{2}\right)\right) \rightarrow O\left(t k N^{2}\right)$.

\section{Análise de Complexidade - Espaço}

A matriz relacional $\mathbf{R}$ requer $O\left(N^{2}\right)$ unidades de espaço. As saídas do algoritmo requerem $O(k N)$ para a matriz de partição e $O(k)$ para os índices dos objetos que são medóides. Portanto, dado que $N>k$, a complexidade de espaço do algoritmo de agrupamento FCMdd é $O\left(N^{2}+k N+k\right) \rightarrow O\left(N^{2}\right)$.

\subsubsection{FCTMdd: Fuzzy c-Trimmed Medoids}

O algoritmo FCMdd considera todos os objetos para determinar o novo medóide a cada passo do algoritmo. Dessa forma, além de um alto custo computacional, um objeto outlier ainda pode levar a uma solução indesejada, apesar do uso de medóides ser mais robusto à outliers. Para evitar esse problema, o algoritmo FCTMdd (Fuzzy c-Trimmed Medoids - em inglês) (Krishnapuram et al., 1999) utiliza apenas uma parcela, s, dos objetos para determinar os novos medóides.

A função objetivo do algoritmo FCMdd em (2.43) foi reescrita usando a função de 
pertinência em (2.45), resultando em:

$$
J=\sum_{j=1}^{N}\left(\sum_{i=1}^{k}\left(r\left(\mathbf{x}_{j}, \mathbf{v}_{i}\right)\right)^{1 /(1-m)}\right)^{(1-m)}=\sum_{j=1}^{N} h_{j}
$$

em que

$$
h_{j}=\left(\sum_{i=1}^{k}\left(r\left(\mathbf{x}_{j}, \mathbf{v}_{i}\right)\right)^{1 /(1-m)}\right)^{(1-m)}
$$

é $1 / k$ vezes a média harmônica das dissimilaridades $\left(r\left(\mathbf{x}_{j}, \mathbf{v}_{i}\right)\right), i=1, \ldots, k$ quando $m=2$. A ideia do algoritmo consiste então em considerar somente os $s$ menores valores de $h_{j}$. Desta forma, o esforço computacional se concentra apenas nos objetos mais próximos dos medóides e, consequentemente, os objetos outliers não são considerados.

O procedimento iterativo do algoritmo FCMTdd é descrito no Algoritmo 15.

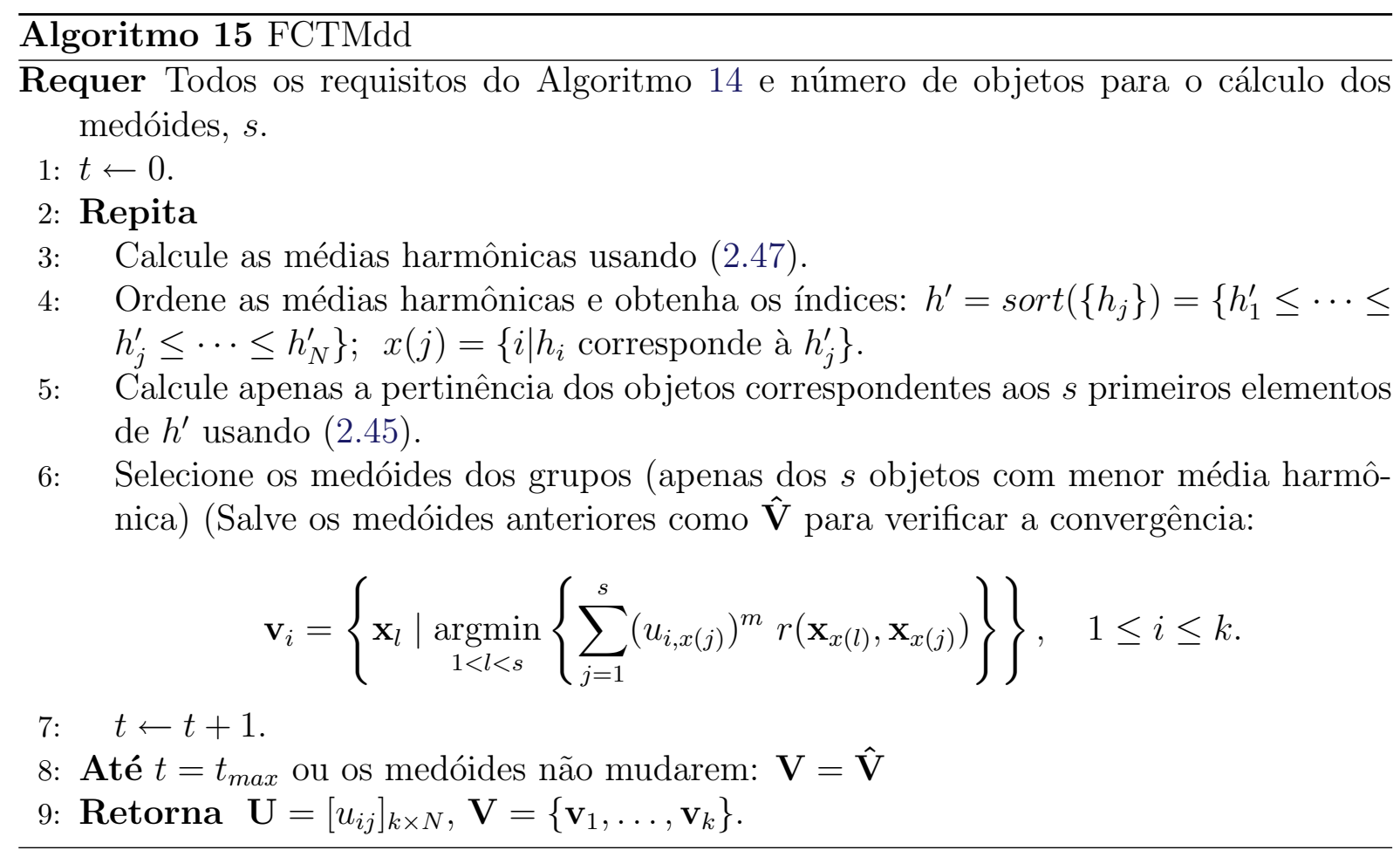

\section{Análise de Complexidade - Tempo}

O cálculo das médias harmônicas usando (2.47) requer $O(k N)$. A ordenação das médias requer $O(N \log N)$. A atualização das pertinências de $s$ elementos em (2.45) requer $O(k s)$ operações, usando o mesmo procedimento adotado por Kolen \& Hutcheson (2002). A seleção dos medóides requer $O\left(k s^{2}\right)$. Portanto, a complexidade de tempo do algoritmo de agrupamento FCTMdd é $O\left(t\left(k N+N \log N+k s+k s^{2}\right)\right) \rightarrow O\left(t\left(k N+N \log N+k s^{2}\right)\right)$. 
A matriz relacional $\mathbf{R}$ requer $O\left(N^{2}\right)$ unidades de espaço. As saídas do algoritmo requerem $O(k N)$ para a matriz de partição e $O(k)$ para os índices dos objetos que são medóides. A matriz de partição de $s$ elementos requer $O(k s)$ unidades de espaço. As médias harmônicas são calculadas e armazenadas em uma tabela à parte, o que requer $O(N)$. Portanto, dado que $N>k$ e $N>s$, a complexidade de espaço do algoritmo de agrupamento FCTMdd é $O\left(N^{2}+k N+k+k s+N\right) \rightarrow O\left(N^{2}\right)$.

\subsubsection{RFCM: Relational Fuzzy c-Means}

O algoritmo RFCM (Relational Fuzzy c-Means — em inglês) (Hathaway et al., 1989) foi elaborado como a versão relacional do algoritmo FCM (Seção 2.2.1). Dessa forma, usando apenas a matriz relacional dos dados, i.e., as distâncias entre os pares de objetos, o algoritmo RFCM resulta na mesma matriz de partição que seria obtida pelo algoritmo FCM usando a matriz dos dados ${ }^{12}$. Em outras palavras, a diferença entre o algoritmo RFCM e o algoritmo FCM está no fato de que o primeiro trabalha apenas com os dados relacionais (matriz relacional $\mathbf{R}$ ) e o segundo com a matriz de dados $\mathbf{X}$.

A função objetivo a ser minimizada pelo algoritmo RFCM é dada por:

$$
J=\sum_{i=1}^{k}\left(\sum_{j=1}^{N} \sum_{c=1}^{N}\left(u_{i j}\right)^{m}\left(u_{i c}\right)^{m} r\left(\mathbf{x}_{j}, \mathbf{x}_{c}\right) /\left(2 \sum_{t=1}^{N} u_{i t}\right)\right) .
$$

Hathaway et al. (1989) demonstraram que minimizar U e V em (2.6) (FCM) é equivalente a minimizar U em (2.48) (RFCM). Dessa forma, se os parâmetros iniciais forem os mesmos para ambos algoritmos, seus passos e seus resultados finais serão idênticos.

Os autores demonstraram também que $D_{i j}$ calculado segundo (2.9) (i.e. usando os protótipos e a matriz de dados) é equivalente a $(1 \leq i \leq k, 1 \leq j \leq N)$

$$
D_{i j}=\left(\mathbf{R} \mathbf{v}_{i}\right)_{j}-\left(\mathbf{v}_{i}^{T} \mathbf{R} \mathbf{v}_{i}\right) / 2
$$

em que os protótipos agora são definidos por $(1 \leq i \leq k)$

$$
\mathbf{v}_{i}=\frac{\left[\left(u_{i 1}\right)^{m}, \ldots,\left(u_{i N}\right)^{m}\right]^{T}}{\sum_{j=1}^{N}\left(u_{i j}\right)^{m}}
$$

em que $\left[\left(u_{i 1}\right)^{m}, \ldots,\left(u_{i N}\right)^{m}\right]^{T}$ é um vetor de $N$ posições, e $\left(\mathbf{R v}_{i}\right)_{j}$ representa o $j$-ésimo elemento do vetor resultante de $\mathbf{R v}_{i}$. Note que os protótipos em (2.50) (RFCM) $\left(\mathbf{v}_{i} \in \mathbb{R}^{N}\right)$ são diferentes dos protótipos em (2.10) $(\mathrm{FCM})\left(\mathbf{v}_{i} \in \mathbb{R}^{n}\right)$. Entretanto, as distâncias calculadas utilizando apenas a matriz relacional segundo (2.49) e (2.50) (RFCM) são idênticas às distâncias calculadas usando a matriz de dados segundo $(2.9)$ e $(2.10)(\mathrm{FCM})^{13}$.

\footnotetext{
${ }^{12}$ Quando a matriz relacional é construída utilizando a distância Euclideana quadrada entre os pares de objetos.

${ }^{13}$ Quando $\mathbf{A}=\mathbf{I}_{n \times n}$, como usualmente utilizado.
} 
Uma vez que $D_{i j}$ dos algoritmos FCM e RFCM são idênticos para todo $i=1, \ldots, k ; j=$ $1, \ldots, N$, é fácil verificar que a cada passo do algoritmo as pertinências calculadas em (2.8) serão idênticas e, consequentemente, o algoritmo RFCM produzirá o mesmo resultado que o algoritmo FCM.

Os passos do algoritmo RFCM estão ilustrados no Algoritmo 16.

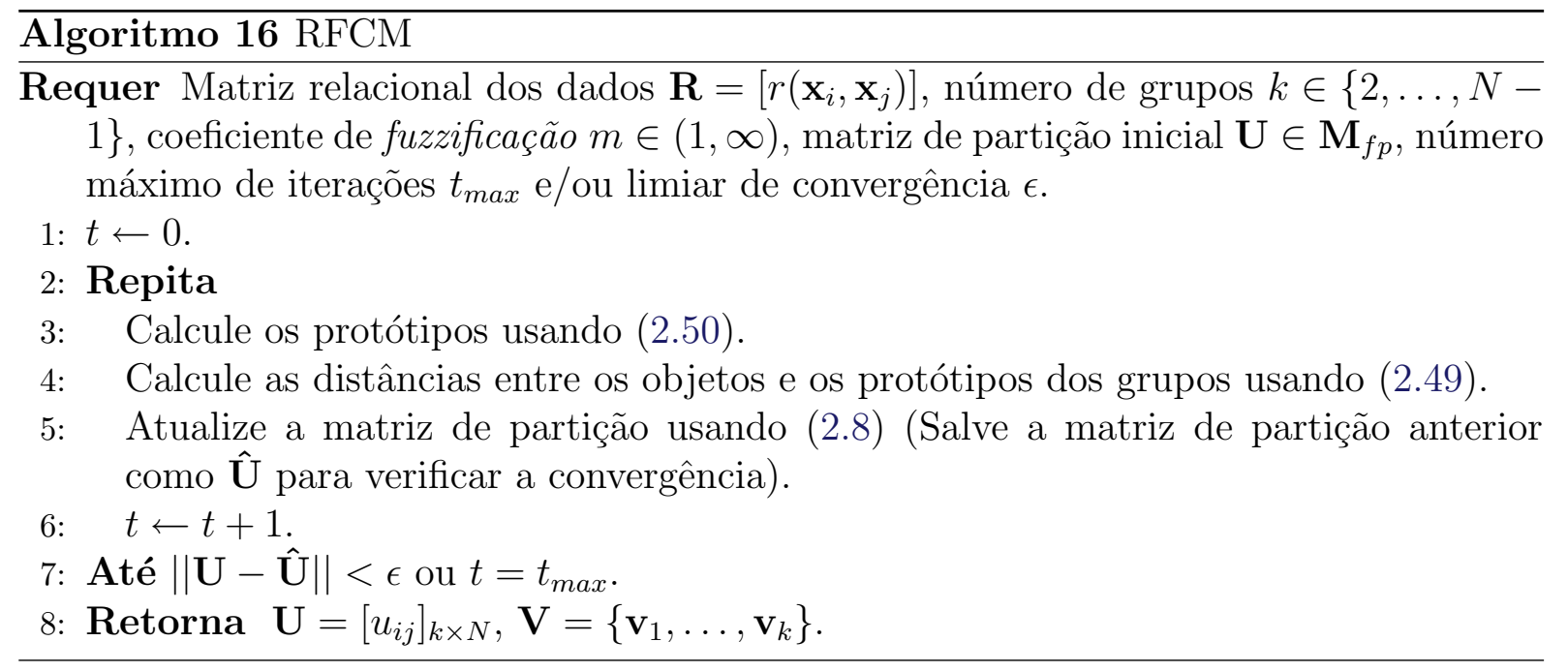

\section{Análise de Complexidade - Tempo}

O cálculo dos protótipos usando (2.50) requer $O(k N)$ operações. O cálculo das distâncias em (2.49) requer $O\left(k N^{2}\right)$. A atualização das pertinências em (2.8) requer $O(k N)$ operações, usando o mesmo procedimento adotado por Kolen \& Hutcheson (2002). Portanto, a complexidade de tempo do algoritmo de agrupamento RFCM é $O\left(t\left(k N+k N^{2}+k N\right)\right) \rightarrow O\left(t k N^{2}\right)$.

\section{Análise de Complexidade - Espaço}

A matriz relacional $\mathbf{R}$ requer $O\left(N^{2}\right)$ unidades de espaço. A matriz de partição requer $O(k N)$ unidades de espaço. Os protótipos requerem $O(k N)$ unidades de espaço. As distâncias são calculadas e armazenadas em uma tabela à parte, o que requer $O(k N)$. Portanto, dado que $N>k$, a complexidade de espaço do algoritmo de agrupamento RFCM é $O\left(N^{2}+k N+k N+k N\right) \rightarrow O\left(N^{2}\right)$.

\subsubsection{NERFCM: Non-Euclidean Relational Fuzzy c-Means}

O algoritmo RFCM pode ser usado para agrupar $N$ objetos de uma base de dados se e somente se existirem $N$ pontos em $\mathbb{R}^{N-1}$ cujas distâncias Euclideanas quadradas formarem exatamente a matriz relacional dos dados $\mathbf{R}=\left[r\left(\mathbf{x}_{i}, \mathbf{x}_{j}\right)\right]$ (Hathaway \& Bezdek, 1994). Essa restrição nas dissimilaridades dos dados pode fazer com que o algoritmo RFCM seja inaplicável em vários cenários de agrupamento de dados relacional. Para contornar esse problema, o algoritmo NERFCM (Non-Euclidean Relational Fuzzy c-Means — em inglês) 
(Hathaway \& Bezdek, 1994) generaliza o algoritmo RFCM para trabalhar com qualquer matriz relacional dos dados que satisfaça às seguintes condições:

1. $r\left(\mathbf{x}_{i}, \mathbf{x}_{i}\right)=0, \quad 1 \leq i \leq N$;

2. $r\left(\mathbf{x}_{i}, \mathbf{x}_{j}\right) \geq 0, \quad 1 \leq i \leq N ; 1 \leq j \leq N$;

3. $r\left(\mathbf{x}_{j}, \mathbf{x}_{i}\right)=r\left(\mathbf{x}_{i}, \mathbf{x}_{j}\right), \quad 1 \leq i \leq N ; 1 \leq j \leq N$.

Se a matriz relacional dos dados $\mathbf{R}$ for Euclideana (i.e. existem $N$ pontos em um espaço $\mathbb{R}^{N-1}$ cujas distâncias Euclideanas quadradas formam exatamente $\mathbf{R}$ ) o algoritmo NERFCM se comporta exatamente como o RFCM. Porém, quando essa restrição não é satisfeita, o algoritmo NERFCM "espalha" as distâncias contidas na matriz relacional dos dados a fim de torná-la Euclideana:

$$
\mathbf{R}=\mathbf{R}+\beta(\mathbf{M}-\mathbf{I}),
$$

em que $\mathbf{R}$ é a matriz relacional dos dados, $\beta$ é um escalar a ser escolhido (automaticamente pelo algoritmo), $\mathbf{I} \in \mathbb{R}^{N \times N}$ é a matriz identidade e $\mathbf{M}=\left[M_{i j}\right] \in \mathbb{R}^{N \times N}$ é a matriz com todos os elementos iguais a 1, i.e.,

$$
M_{i j}=1, \quad 1 \leq i, j \leq N .
$$

Mais precisamente, se alguma distância $D_{i j}$ em (2.49) for negativa, i.e., $D_{i j}<0$ para qualquer $i, j$, então o valor de $\beta$ (que inicialmente é nulo) deve ser modificado:

$$
\beta=\max \left\{-2 D_{i j} /\left(\left\|\mathbf{v}_{i}-\mathbf{e}_{j}\right\|^{2}\right) \mid \forall D_{i j}<0\right\}
$$

e então, para conter apenas distâncias positivas, todas as distâncias devem ser modificadas $(1 \leq i \leq k ; 1 \leq j \leq N)$ :

$$
D_{i j}=D_{i j}+(\beta / 2)\left\|\mathbf{v}_{i}-\mathbf{e}_{j}\right\|^{2}
$$

em que $\mathbf{e}_{j}$ é um vetor de $N$ posições cujo $j$-ésimo elemento vale 1 e os demais elementos valem 0 , i.e., $\mathbf{e}_{j}=\left[e_{c j}\right] \in \mathbb{R}^{N}$ tal que $e_{c j}=0$ para $c \neq j$ e $e_{c j}=1$ para $c=j$.

Os passos do algoritmo NERFCM estão ilustrados no Algoritmo 17.

\section{Análise de Complexidade - Tempo}

A análise de complexidade do algoritmo NERFCM segue da análise do algoritmo RFCM (Seção 2.2.6.3). Os cálculos em (2.52) e (2.53) não alteram essa complexidade. Portanto, a complexidade de tempo do algoritmo de agrupamento NERFCM é $O\left(t k N^{2}\right)$. 


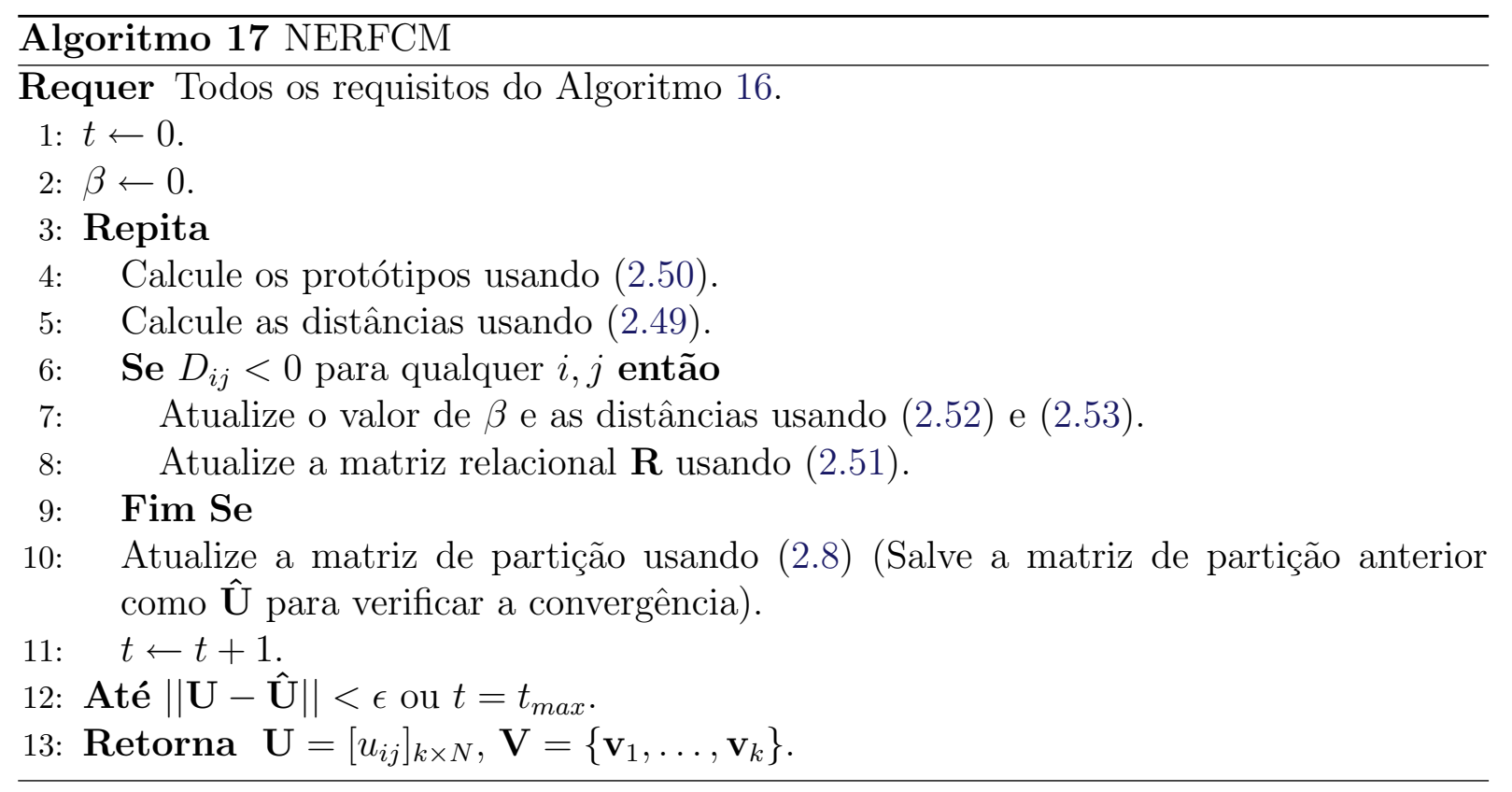

\section{Análise de Complexidade - Espaço}

A análise de complexidade do algoritmo NERFCM segue da análise do algoritmo RFCM (Seção 2.2.6.3). Os cálculos em (2.52) e (2.53) não alteram essa complexidade. Portanto, a complexidade de espaço do algoritmo de agrupamento NERFCM é $O\left(N^{2}\right)$.

\subsubsection{Algoritmos que Lidam com Valores Ausentes}

Existem cenários nos quais alguns valores na matriz de dados $\mathbf{X}=\left[\mathbf{x}_{j}\right]$ não estão definidos. Tais valores são denominados valores ausentes e podem ser resultado de fenômenos tais como: problemas com o aparelho de medição de determinado atributo, cliente se recusa a informar determinado valor de atributo (e.g. salário), entre outros.

Tome como exemplo a matriz de dados ilustrada na Tabela 2.7. Considerando que o símbolo "?" representa um valor ausente, é possível identificar três valores ausentes nessa matriz de dados. Os valores de salário, idade e altura dos Objetos 2, 3 e 5, respectivamente, estão ausentes.

$\mathbf{X}=$\begin{tabular}{|c|c|c|c|}
\hline & Idade & Salário & Altura \\
\hline Objeto 1 & 21 & $\mathrm{R} \$ 830,00$ & 1,81 \\
\hline Objeto 2 & 45 & $?$ & 1,74 \\
\hline Objeto 3 & $?$ & $\mathrm{R} \$ 1205,00$ & 1,75 \\
\hline Objeto 4 & 31 & $\mathrm{R} \$ 1530,00$ & 1,90 \\
\hline Objeto 5 & 42 & $\mathrm{R} \$ 1771,00$ & $?$ \\
\hline \hline
\end{tabular}

Tabela 2.7: Matriz de dados com cinco objetos descritos por três atributos.

Uma das abordagens utilizadas para "tratar" valores ausentes consiste em aplicar métodos de imputação de valores ausentes na etapa de pré-processamento dos dados (e.g., 
veja (Silva, 2010)). Entretanto, alguns algoritmos de agrupamento de dados foram desenvolvidos para trabalhar naturalmente com valores ausentes e são apresentados a seguir.

\subsubsection{WDSFCM: Whole Data Strategy Fuzzy c-Means}

O algoritmo WDSFCM ( Whole Data Strategy Fuzzy c-Means - em inglês) (Hathaway \& Bezdek, 2001) consiste simplesmente em eliminar os objetos que apresentarem valores ausentes e, então, aplicar o algoritmo FCM (Seção 2.2.1). Dessa forma, o algoritmo FCM é aplicado a apenas um subconjunto dos objetos. Por exemplo, para a matriz de dados original ilustrada na Tabela 2.7, o algoritmo FCM seria aplicado na matriz de dados ilustrada na Tabela 2.8 .

$\mathbf{X}^{\prime}=$\begin{tabular}{|c|c|c|c|}
\hline & Idade & Salário & Altura \\
\hline Objeto 1 & 21 & $\mathrm{R} \$ 830,00$ & 1,81 \\
\hline Objeto 4 & 31 & $\mathrm{R} \$ 1530,00$ & 1,90 \\
\hline
\end{tabular}

Tabela 2.8: Matriz de dados com dois objetos descritos por três atributos.

Note que os Objetos 2, 3 e 5, que apresentavam ao menos um valor ausente em algum dos atributos, foram eliminados e o algoritmo FCM seria aplicado somente com os Objetos 1 e 4. Devido à perda de informação, uma vez que todos os demais atributos dos objetos eliminados também são perdidos, os autores sugerem que o algoritmo WDSFCM seja utilizado apenas em cenários nos quais o número de valores ausentes não seja maior que $25 \%$ do total da matriz de dados. Note também que a execução do algoritmo FCM com um subconjunto dos objetos, apesar de resultar em uma boa aproximação para os protótipos, implica nas pertinências dos objetos eliminados para os grupos não serem diretamente encontradas pelo algoritmo. Tais pertinências podem, entretanto, ser estimadas utilizando um cálculo de distância parcial (e.g. Seção 2.2.7.2) entre os objetos eliminados e os centros encontrados.

\section{Análise de Complexidade - Tempo}

A análise de complexidade de tempo do algoritmo WDSFCM deriva da análise do algoritmo FCM (Seção 2.2.1). Portanto a complexidade de tempo do algoritmo de agrupamento WDSFCM é $O\left(t k N n^{2}\right)$. Quando A é a matriz identidade a complexidade é $O(t k N n)$.

\section{Análise de Complexidade - Espaço}

A análise de complexidade de espaço do algoritmo WDSFCM deriva da análise do algoritmo FCM. Portanto, a complexidade de espaço do algoritmo de agrupamento WDSFCM é $O\left(N n+k N+n^{2}\right)$. Quando $\mathbf{A}$ é a matriz identidade a complexidade de espaço é $O(N n+k N)$. 


\subsubsection{PDSFCM: Partial Distance Strategy Fuzzy c-Means}

O algoritmo PDSFCM (Partial Distance Strategy Fuzzy c-Means - em inglês) (Hathaway \& Bezdek, 2001) adota uma distância modificada que pode ser utilizada por objetos que apresentam valores ausentes em alguns dos atributos. A distância (modificada) entre o objeto $\mathbf{x}_{j}$ e o protótipo $\mathbf{v}_{i}$ é dada por:

$$
D_{i j}=\left\|\mathbf{x}_{j}-\mathbf{v}_{i}\right\|^{2}=\frac{n}{I_{j}} \sum_{p=1}^{n}\left(x_{j p}-v_{i p}\right)^{2} I_{j p},
$$

em que $x_{j p}$ é o $p$-ésimo atributo do objeto $\mathbf{x}_{j}, v_{i p}$ é o $p$-ésimo atributo do protótipo $\mathbf{v}_{i}$,

$$
I_{j p}=\left\{\begin{array}{ll}
0, & \text { se o } p \text {-ésimo atributo do } j \text {-ésimo objeto for ausente. } \\
1, & \text { caso contrário. }
\end{array},\right.
$$

e $I_{j}=\sum_{p=1}^{n} I_{j p}$.

Por exemplo, a distância entre um dado protótipo $v_{1}=(25,1000,1.80)$ e o Objeto 2 da Tabela 2.7 é dada por:

$$
D_{12}=\|(45, ?, 1.74)-(25,1000,1.80)\|^{2}=\frac{3}{2}\left[(45-25)^{2}+(1.74-1.80)^{2}\right] .
$$

Assim, o algoritmo PDSFCM minimiza (2.6) com as distâncias dadas por (2.54). As condições necessárias para minimizar (2.6), com $D_{i j}$ dada por (2.54), são (2.8) e $(1 \leq i \leq k ; 1 \leq p \leq n)$

$$
v_{i p}=\frac{\sum_{j=1}^{N}\left(u_{i j}\right)^{m} I_{j p} x_{j p}}{\sum_{j=1}^{N}\left(u_{i j}\right)^{m} I_{j p}} .
$$

O procedimento iterativo do algoritmo PDSFCM é descrito no Algoritmo 18.

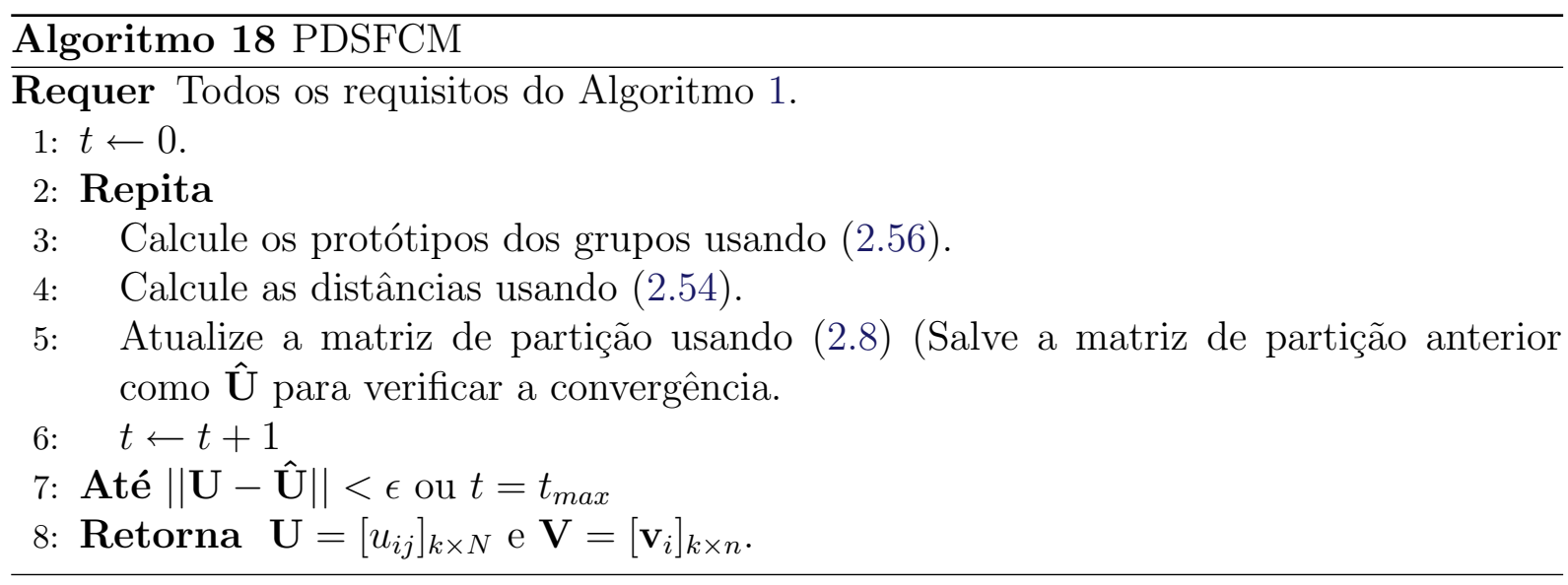

\section{Análise de Complexidade - Tempo}

O cálculo dos protótipos em (2.56) requer $O(k N n)$ operações. O cálculo das distâncias em (2.54) requer $O(k N n)$ operações. A atualização das pertinências em (2.8) requer 
$O(k N)$ operações, usando o mesmo procedimento adotado por Kolen \& Hutcheson (2002). Portanto, a complexidade de tempo do algoritmo de agrupamento PDSFCM é $O(t(k N n+$ $k N n+k N)) \rightarrow O(t k N n)$.

\section{Análise de Complexidade - Espaço}

A base de dados $\mathbf{X}$ requer $O(N n)$ unidades de espaço. As saídas do algoritmo requerem $O(k N)$ para a matriz de partição e $O(k n)$ para os protótipos dos grupos. As distâncias são calculadas e armazenadas em uma tabela à parte, o que requer $O(k N)$ unidades de espaço. Portanto, dado que $N>k$, a complexidade de espaço do algoritmo de agrupamento PDSFCM é $O(N n+k N+k n+k N) \rightarrow O(N n+k N)$.

\subsubsection{OCSFCM: Optimal Completion Strategy Fuzzy c-Means}

O algoritmo OCSFCM (Optimal Completion Strategy Fuzzy c-Means - em inglês) (Hathaway \& Bezdek, 2001) considera os valores ausentes como variáveis adicionais no problema de minimização. Portanto, tais variáveis (valores ausentes) são obtidas pelo próprio problema de otimização, que procura os valores mais adequados para que a função objetivo seja minimizada.

Considere o subconjunto de objetos e atributos que apresentam valores ausentes $\mathbf{X}_{a} \subset \mathbf{X}$, em que $\mathbf{X}_{a}=\left\{x_{j p}=\right.$ ? para $1 \leq j \leq N, 1 \leq p \leq n$, tal que o valor de $x_{j p}$ é ausente em $\mathbf{X}\}$. Dessa forma, minimizar (2.6) em relação a $x_{j p} \in \mathbf{X}_{a}$ leva a:

$$
x_{j p}=\frac{\sum_{i=1}^{k}\left(u_{i j}\right)^{m} v_{i p}}{\sum_{i=1}^{k}\left(u_{i j}\right)^{m}}, \quad \forall x_{j p} \in \mathbf{X}_{a},
$$

i.e., a cada iteração do algoritmo, as variáveis referentes aos valores ausentes são recalculadas como a média ponderada (pelos valores de pertinência) dos protótipos ${ }^{14}$.

O procedimento iterativo do algoritmo OCSFCM é descrito no Algoritmo 19.

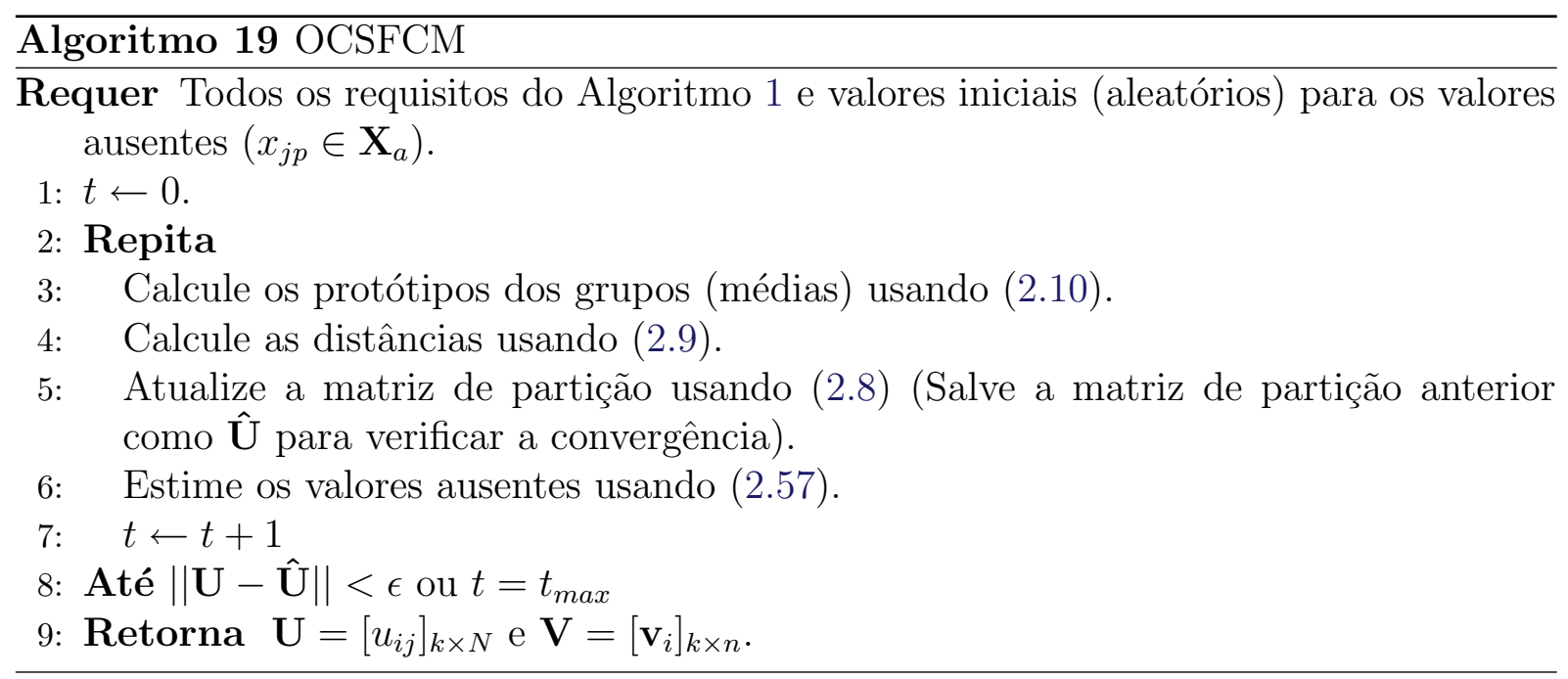

\footnotetext{
${ }^{14}$ No passo inicial essas variáveis podem ser iniciadas aleatoriamente.
} 


\section{Análise de Complexidade - Tempo}

O cálculo dos protótipos em (2.10) requer $O(k N n)$ operações. O cálculo das distâncias em (2.9) requer $O\left(k N n^{2}\right)$ operações. A atualização das pertinências em (2.8) requer $O(k N)$ operações, usando o mesmo procedimento adotado por Kolen \& Hutcheson (2002). A estimativa dos valores ausentes em (2.57) requer $O(k N n)$. Portanto, a complexidade de tempo do algoritmo de agrupamento OCSFCM é $O\left(t\left(k N n+k N n^{2}+k N+k N n\right)\right) \rightarrow$ $O\left(t k N n^{2}\right)$. Quando A é a matriz identidade a complexidade é $O(t k N n)$.

\section{Análise de Complexidade - Espaço}

A base de dados $\mathbf{X}$ requer $O(N n)$ unidades de espaço. As saídas do algoritmo requerem $O(k N)$ para a matriz de partição e $O(k n)$ para os protótipos dos grupos. Para calcular as distâncias é necessário armazenar $\mathbf{A}$, o que requer $O\left(n^{2}\right)$. As distâncias são calculadas e armazenadas em uma tabela à parte, o que requer $O(k N)$ unidades de espaço. Portanto, dado que $N>k$, a complexidade de espaço do algoritmo de agrupamento OCSFCM é $O\left(N n+k N+k n+n^{2}+k N\right) \rightarrow O\left(N n+k N+n^{2}\right)$. Quando A é a matriz identidade a complexidade de espaço é $O(N n+k N)$.

\subsubsection{NPSFCM: Nearest Prototype Strategy Fuzzy c-Means}

O algoritmo NPSFCM (Nearest Prototype Strategy Fuzzy c-Means — em inglês) (Hathaway \& Bezdek, 2001) é similar ao algoritmo OCSFCM (Seção 2.2.7.3). A única diferença está na forma em que os valores ausentes são estimados. Enquanto o algoritmo OCSFCM estima os valores ausentes calculando uma média ponderada (pelas pertinências) dos protótipos, o algoritmo NPSFCM, por sua vez, estima-os a partir dos valores do protótipo mais próximo (de acordo com a distância parcial em (2.54)), i.e., $\left(\forall x_{j p} \in \mathbf{X}_{a}\right.$ )

$$
x_{j p}=v_{i p} \text { tal que } D_{i j}=\min \left\{D_{1 j}, \cdots, D_{k j}\right\} .
$$

O procedimento iterativo do algoritmo NPSFCM é descrito no Algoritmo 20.

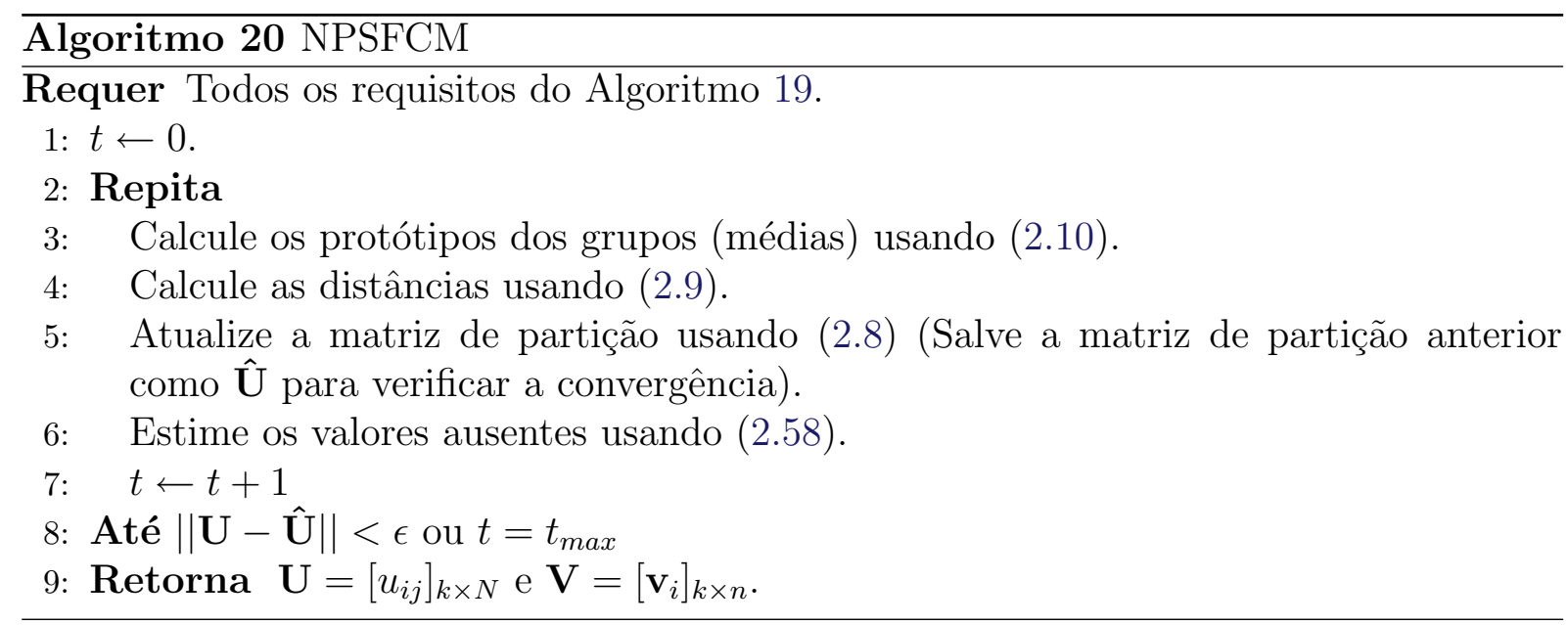


O cálculo dos protótipos em (2.10) requer $O(k N n)$ operações. O cálculo das distâncias em (2.9) requer $O\left(k N n^{2}\right)$ operações. A atualização das pertinências em (2.8) requer $O(k N)$ operações, usando o mesmo procedimento adotado por Kolen \& Hutcheson (2002). A estimação dos valores ausentes em (2.58) requer $O(k N n)$. Portanto, a complexidade de tempo do algoritmo de agrupamento NPSFCM é $O\left(t\left(k N n+k N n^{2}+k N+k N n\right)\right) \rightarrow$ $O\left(t k N n^{2}\right)$. Quando A é a matriz identidade a complexidade é $O(t k N n)$.

\section{Análise de Complexidade - Espaço}

A base de dados $\mathbf{X}$ requer $O(N n)$ unidades de espaço. As saídas do algoritmo requerem $O(k N)$ para a matriz de partição e $O(k n)$ para os protótipos dos grupos. Para calcular as distâncias é necessário armazenar A, o que requer $O\left(n^{2}\right)$. As distâncias são calculadas e armazenadas em uma tabela à parte, o que requer $O(k N)$ unidades de espaço. Portanto, dado que $N>k$, a complexidade de espaço do algoritmo de agrupamento NPSFCM é $O\left(N n+k N+k n+n^{2}+k N\right) \rightarrow O\left(N n+k N+n^{2}\right)$. Quando A é a matriz identidade a complexidade de espaço é $O(N n+k N)$.

\subsubsection{Algoritmos que Buscam Diminuir o Tempo de Processamento}

A execução do algoritmo FCM (Seção 2.2.1) em grandes bases de dados pode demandar uma grande quantidade de tempo. Uma maneira de executar o algoritmo mais rapidamente consiste em executá-lo paralelamente (ou de maneira distribuída) em vários processadores (ou computadores). Essa abordagem será discutida em detalhes no Capítulo 3.

Uma abordagem alternativa para o mesmo problema consiste em aplicar modificações no algoritmo FCM original com o objetivo de diminuir o tempo de execução do algoritmo, mesmo que o resultado final não seja exatamente o mesmo. Nesta seção são apresentadas algumas versões propostas para diminuir o esforço computacional do algoritmo FCM.

\subsubsection{1 brFCM}

Apesar de não ser muito comum, alguns cenários podem apresentar vários objetos com os mesmos valores. Tome como exemplo uma matriz de pixel $800 \times 600$ em escalas de cinza (valores variando de 0 a 255). Se o objetivo é agrupar pixels descritos por tons de cinza similares, então é possível reduzir $800 \times 600=480,000$ pixels para apenas 256 (tons de cinza), em que cada tom apresenta um peso proporcional à quantidade de pixels com aquele tom em específico ${ }^{15}$.

Formalmente, considerando $N^{0}<<N$ o número de objetos distintos da base de dados, se $N^{0}$ for muito menor do que $N$ então a execução do algoritmo FCM utilizando apenas

\footnotetext{
${ }^{15}$ Exemplo adaptado de (Eschrich et al., 2003).
} 
os $N^{0}$ objetos pode apresentar uma redução significativa no esforço computacional. Entretanto, é necessário que cada objeto tenha um peso, $w_{j}$, proporcional ao número de objetos idênticos (que apresentam as mesmas características).

Dessa forma, no algoritmo brFCM (Eschrich et al., 2003) os autores propuseram calcular os protótipos dos grupos como $(1 \leq i \leq k)$ :

$$
\mathbf{v}_{i}=\frac{\sum_{j=1}^{N^{0}} w_{j}\left(u_{i j}\right)^{m} \mathbf{x}_{j}^{\prime}}{\sum_{j=1}^{N^{0}} w_{j}\left(u_{i j}\right)^{m}}
$$

em que $\mathbf{x}_{j}^{\prime}$ representa o $j$-ésimo elemento da matriz de dados $\mathbf{X}^{\prime}=\left[\mathbf{x}_{j}\right] \in \mathbb{R}^{N^{0} \times n}$, contendo apenas os $N^{0}$ objetos distintos de $\mathbf{X}$, i.e., $\mathbf{X}^{\prime} \subseteq \mathbf{X}$, e $w_{j}$ é a quantidade de objetos (na matriz de dados original $\mathbf{X}$ ) que são representados pelo elemento $\mathbf{x}_{j}^{\prime}$. As pertinências são atualizadas de acordo com (2.8) utilizando apenas os objetos em $\mathbf{X}^{\prime}$, i.e.,

$$
u_{i j}^{\prime}=\left(\sum_{c=1}^{k}\left(\frac{D_{i j}^{\prime}}{D_{c j}^{\prime}}\right)^{1 /(m-1)}\right)^{-1}, 1 \leq i \leq k, 1 \leq j \leq N^{0}
$$

com as distâncias dadas por $\left(1 \leq i \leq k, 1 \leq j \leq N^{0}\right)$ :

$$
D_{i j}^{\prime}=\left\|\mathbf{x}_{j}^{\prime}-\mathbf{v}_{i}\right\|_{\mathbf{A}}^{2}=\left(\mathbf{x}_{j}^{\prime}-\mathbf{v}_{i}\right)^{T} \mathbf{A}\left(\mathbf{x}_{j}^{\prime}-\mathbf{v}_{i}\right)
$$

Todos os objetos em $\mathbf{X}$ que passam a ser representados pelo mesmo elemento $\mathbf{x}_{j}^{\prime}$ recebem a mesma pertinência.

Essa abordagem funciona bem para bases de dados que apresentam objetos idênticos. Mais especificamente, executar o algoritmo brFCM ou o algoritmo FCM em tal base de dados produz a mesma matriz de partição final. Entretanto, bases com objetos idênticos não são muito comuns. Eschrich et al. (2003) propuseram, então, um passo de quantização ao algoritmo. A quantização reduz a precisão de representação dos objetos e, dependendo dessa redução, faz com que mais ou menos objetos sejam representados pelos mesmos valores. Tome como exemplo quatro objetos ilustrados na Tabela $2.9^{16}$. Os objetos são descritos por apenas um único atributo numérico ilustrado na segunda coluna da Tabela 2.9. A representação em binário desse atributo é ilustrada na terceira coluna da tabela. Suponha que seja atribuído um nível de quantização nos três bits menos significativos da representação binária dos objetos. Isso significa que tais bits serão substituídos por zeros, conforme ilustrado na quarta coluna da Tabela 2.9. Portanto, após o passo de quantização dos objetos, eles serão descritos pela quinta coluna da Tabela 2.9. Note que os Objetos 1 e 2, que apresentavam valores 187 e 184, respectivamente, serão representados por apenas um único objeto na matriz $\mathbf{X}^{\prime}$, com valor 184. O mesmo ocorre com os Objetos 3 e 4, que apresentavam valores 103 e 99, respectivamente, mas serão representados por apenas um único objeto com valor 96.

Depois que os objetos são quantizados espera-se que o número de objetos distintos

\footnotetext{
${ }^{16}$ Exemplo adaptado de (Eschrich et al., 2003).
} 


\begin{tabular}{|l|c|c|c|c|}
\hline & Atributo & $\begin{array}{c}\text { Atributo } \\
\text { (em binário) }\end{array}$ & $\begin{array}{c}\text { Atributo quantizado } \\
\text { (em binário) }\end{array}$ & $\begin{array}{c}\text { Atributo } \\
\text { quantizado }\end{array}$ \\
\hline Objeto 1 & 187 & 10111011 & 10111000 & 184 \\
\hline Objeto 2 & 184 & 10111000 & 10111000 & 184 \\
\hline Objeto 3 & 103 & 01100111 & 01100000 & 96 \\
\hline Objeto 4 & 99 & 01100011 & 01100000 & 96 \\
\hline \hline
\end{tabular}

Tabela 2.9: Quatro objetos representados por apenas um atributo numérico. Representação binária e quantizada também estão ilustradas na tabela.

seja pequeno em relação ao número total de objetos, i.e., espera-se que $N^{0}<<N$. Então o algoritmo é aplicado à base quantizada $\mathbf{X}^{\prime}$.

Os passos do algoritmo brFCM, com o passo de quantização, estão ilustrados no Algoritmo 21.

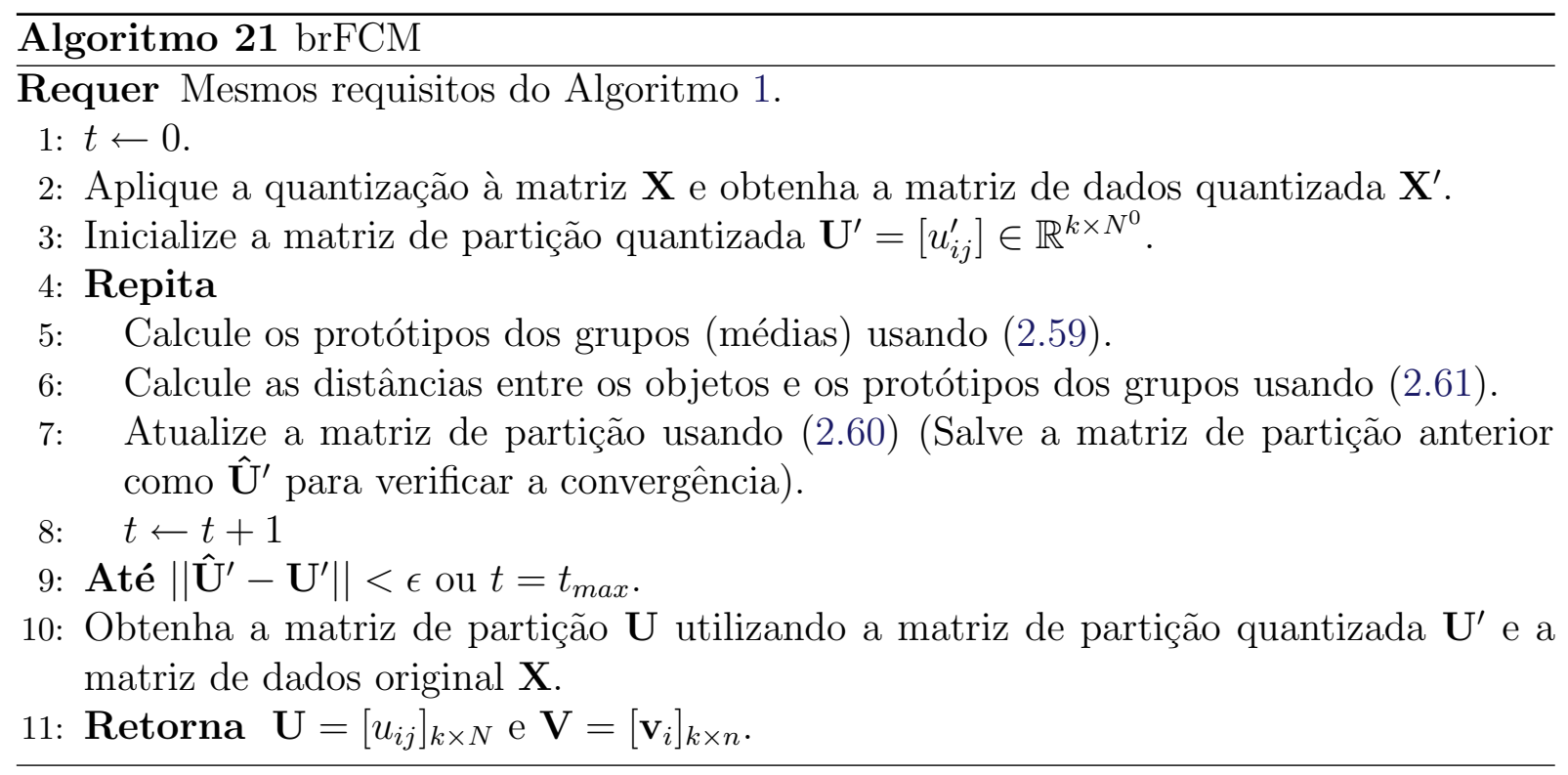

\section{Análise de Complexidade - Tempo}

Aplicar a quantização à matriz $\mathbf{X}$ requer $O(N n+n N \log N)$ operações. Inicializar a matriz de partição $\mathbf{U}^{\prime}$ requer $O\left(k N^{0}\right)$. O cálculo dos protótipos em (2.59) requer $O\left(k N^{0} n\right)$ operações. O cálculo das distâncias em (2.61) requer $O\left(k N^{0} n^{2}\right)$ operações. A atualização das pertinências em (2.60) requer $O\left(k N^{0}\right)$ operações, usando o mesmo procedimento adotado por Kolen \& Hutcheson (2002). Obter a matriz U utilizando $\mathbf{U}^{\prime}$ requer $O(k N)$. Portanto, a complexidade de tempo do algoritmo de agrupamento brFCM é $\left.O\left(N n+n N \log N+k N^{0}+t\left(k N^{0} n+k N^{0} n^{2}+k N^{0}\right)+k N\right)\right) \rightarrow O\left(n N \log N+t k N^{0} n^{2}+k N\right)$. Quando A é a matriz identidade a complexidade é $\left.O\left(n N \log N+t k N^{0} n+k N\right)\right)$. Note que se $N^{0}<<N$ e o algoritmo demandar muitas iterações, $t$, então é esperado que o algoritmo brFCM execute mais rapidamente que o algoritmo FCM. 
A base de dados $\mathbf{X}$ requer $O(N n)$ unidades de espaço. A matriz de dados quantizada requer $O\left(N^{0} n\right)$. As saídas do algoritmo requerem $O(k N)$ para a matriz de partição e $O(k n)$ para os protótipos dos grupos. As distâncias são calculadas e armazenadas em uma tabela à parte, o que requer $O\left(k N^{0}\right)$ unidades de espaço. A matriz de partição $\mathbf{U}^{\prime}$ requer $O\left(k N^{0}\right)$. Portanto, dado que $N>k$ e $N>N^{0}$, a complexidade de espaço do algoritmo de agrupamento brFCM é $O\left(N n+N^{0} n+k N+k n+k N^{0}+k N^{0}\right) \rightarrow O(N n+k N)$.

\subsubsection{2 mrFCM: Multistage Random Sampling Fuzzy c-Means}

Como já vimos, o tempo de execução do algoritmo FCM em uma base de dados com muitos objetos pode ser muito alto. Uma forma de acelerar o algoritmo consiste em, primeiro executá-lo em apenas uma amostra aleatória dos dados para obter protótipos iniciais, e então executá-lo novamente com todos os dados originais e os protótipos encon$\operatorname{trados}^{17}$. Baseado nessa ideia, o algoritmo mrFCM (Multistage Random Sampling Fuzzy c-Means - em inglês) (Cheng et al., 1998) consiste de duas fases. Na primeira, são criadas $r$ amostras do conjunto de dados. A cada amostragem, uma porcentagem $\Delta_{\%}$ de objetos é adicionada à amostragem anterior e o algoritmo FCM é executado. Na segunda fase, o algoritmo FCM é aplicado a toda a base de dados com os protótipos iniciais encontrados na primeira fase.

Formalmente, considere $\mathbf{X}_{\Delta_{\%}}^{s}$ uma amostragem aleatória de $\Delta_{\%} \cdot s$ objetos da matriz de dados $\mathbf{X}$, i.e., $\mathbf{X}_{\Delta_{\%}}^{s} \in \mathbb{R}^{\left(\Delta_{\%} \cdot s\right) \times n}$. O algoritmo mrFCM executa então o algoritmo FCM (Algoritmo 1 na Seção 2.2.1) em $\mathbf{X}_{\Delta \%}^{1}$ (i.e., uma amostragem aleatória de tamanho $\Delta_{\%}$ ) para obter uma aproximação dos protótipos $\left(\mathbf{V}^{1}\right)$. Então, uma nova amostragem de tamanho $\Delta_{\%}$ é feita e adicionada a $\mathbf{X}_{\Delta_{\%}}^{1}$, resultando em $\mathbf{X}_{\Delta_{\%}}^{2}$ que consiste, portanto, de uma amostragem de tamanho $\Delta_{\%} \cdot 2$. Os protótipos encontrados pela execução do algoritmo FCM em $\mathbf{X}_{\Delta_{\%}}^{1}$ (i.e., $\mathbf{V}^{1}$ ) são então usados como protótipos iniciais para a execução do algoritmo $\mathrm{FCM}$ em $\mathbf{X}_{\Delta_{\%}}^{2}$ visando encontrar uma nova aproximação dos protótipos $\mathbf{V}^{2}$. Esse processo se repete $r$ vezes e então o algoritmo FCM é aplicado a toda a base de dados $\mathbf{X}$ com os protótipos iniciais $\mathbf{V}^{r}$.

Os autores propuseram também que o valor de $\epsilon$ (utilizado para análise de convergência) mudasse a cada iteração da primeira etapa do algoritmo. Dessa forma, uma primeira execução poderia apresentar um valor de $\epsilon_{i n i}$ maior para obter uma aproximação dos protótipos mais rapidamente, entretanto esse valor seria decrescido até se tornar um valor tão pequeno quanto desejado $\epsilon_{\text {fim }}$ para obter uma precisão maior nos resultados.

Os passos do algoritmo mrFCM estão descritos no Algoritmo 22.

\footnotetext{
${ }^{17}$ Espera-se que esses protótipos iniciais necessitem de poucas iterações para que o algoritmo convirja e, consequentemente, espera-se que o algoritmo FCM seja executado em poucas iterações com os dados originais.
} 


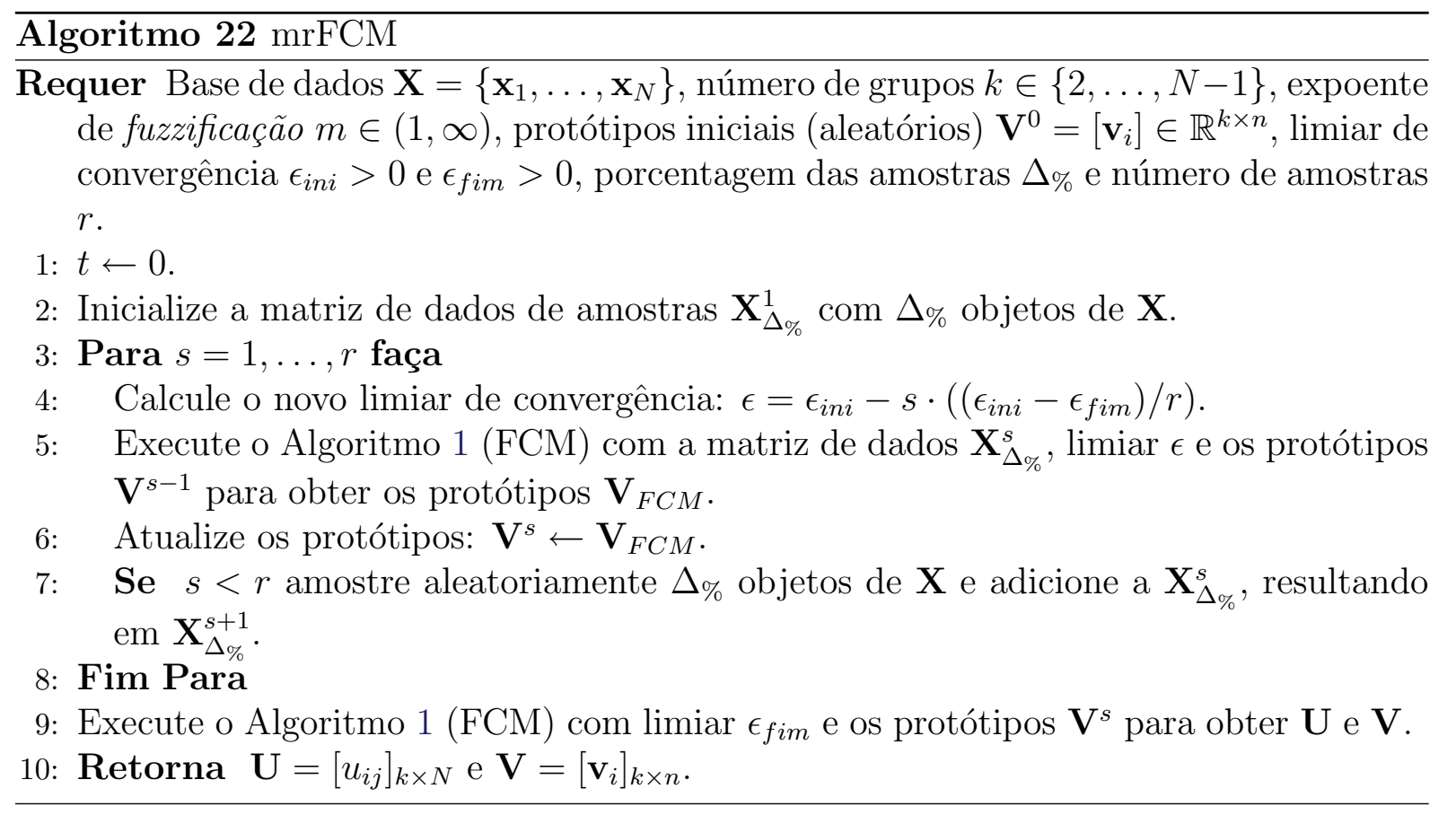

\section{Análise de Complexidade - Tempo}

A inicialização da matriz de dados das amostras requer $O\left(\Delta_{\%} n\right)$. A execução do algoritmo FCM no Passo 5 do Algoritmo 22 requer $O\left(t k s \Delta_{\%} n^{2}\right)$. Dado que o algoritmo é executado $r$ vezes e o tamanho da matriz de amostras cresce até $\Delta_{\%} r$ a complexidade dos Passos 3 até 8 é $O\left(t k \Delta_{\%} r^{2} n^{2}\right)$. Por fim, executar o algoritmo FCM no Passo 9 requer $O\left(t k N n^{2}\right)$. Portanto, a complexidade de tempo do algoritmo de agrupamento mrFCM é $O\left(\Delta_{\%} n+t k \Delta_{\%} r^{2} n^{2}+t k N n^{2}\right) \rightarrow O\left(t k \Delta_{\%} r^{2} n^{2}+t k N n^{2}\right)$. Quando A é a matriz identidade a complexidade de tempo é $O\left(t k \Delta_{\%} r^{2} n+t k N n\right)$. É esperado que a quantidade de iterações do algoritmo FCM em cada amostra seja muito menor do que a quantidade de iterações que seria obtida executando o algoritmo na base de dados original. Assim, dependendo da quantidade de amostras, $r$, e de sua porcentagem, $\Delta_{\%}$, é esperado que a quantidade de iterações total do algoritmo mrFCM seja menor do que a quantidade de iterações que seria obtida executando o algoritmo FCM e, consequentemente, é esperado que o algoritmo mrFCM execute mais rapidamente que o algoritmo FCM.

\section{Análise de Complexidade - Espaço}

A base de dados $\mathbf{X}$ requer $O(N n)$ unidades de espaço. A matriz de dados das amostras requer $O\left(\Delta_{\%} r n\right)$. A execução do algoritmo FCM requer $O\left(N n+k N+n^{2}\right)$. As saídas do algoritmo requerem $O(k N)$ para a matriz de partição e $O(k n)$ para os protótipos dos grupos. Portanto, dado que $N>k$ e $N>\Delta_{\%} r$, a complexidade de espaço do algoritmo de agrupamento mrFCM é $O\left(N n+\Delta_{\%} r n+N n+k N+n^{2}+k N+k n\right) \rightarrow O\left(N n+k N+n^{2}\right)$. Quando A é a matriz identidade a complexidade de espaço é $O(N n+k N)$. 


\subsubsection{3 linear-FCM}

A complexidade de tempo do algoritmo FCM é quadrática em relação ao número de grupos $k$. Mais precisamente, a complexidade é dada por $O\left(k^{2} N n^{2}\right)$. Kolen \& Hutcheson (2002) apresentaram uma maneira de diminuir essa complexidade para $O\left(k N n^{2}\right)$, i.e., uma complexidade linear em relação ao número de grupos $k$.

Note que para atualizar as pertinências

$$
u_{i j}=\left(\sum_{c=1}^{k}\left(\frac{D_{i j}}{D_{c j}}\right)^{1 /(m-1)}\right)^{-1}, 1 \leq i \leq k, 1 \leq j \leq N
$$

o termo

$$
\varphi_{j}=\sum_{c=1}^{k}\left(\frac{1}{D_{c j}}\right)^{1 /(m-1)}, 1 \leq j \leq N,
$$

pode ser calculado para cada objeto antes de calcular $u_{i j}$ em (2.62). Assim, (2.62) se reduz a $(1 \leq i \leq k, 1 \leq j \leq N)$

$$
u_{i j}=\left(\left(D_{i j}\right)^{1 /(m-1)} \cdot \varphi_{j}\right)^{-1}
$$

que requer apenas $O(k N)$ operações para calcular as pertinências de todos os objetos e grupos. Dado que a complexidade do cálculo das distâncias em $(2.9)$ é $O\left(k N n^{2}\right)$, a complexidade do algoritmo passa a ser $O\left(k N n^{2}\right)$ ou $O(k N n)$ quando A é a matriz identidade (como usual).

\section{Análise de Complexidade - Tempo}

O algoritmo linear-FCM consiste basicamente dos mesmos passos do algoritmo FCM (Seção 2.2.1). Porém, o cálculo das pertinências é efetuada utilizando (2.64). Dessa forma a complexidade de tempo é dada por $O\left(t k N n^{2}\right)$. Quando A é a matriz identidade a complexidade de tempo é $O(t k N n)$. Note que esse procedimento foi utilizado nos demais algoritmos deste capítulo a fim de reduzir a complexidade de tempo.

\section{Análise de Complexidade - Espaço}

A análise de complexidade de espaço do algoritmo linear-FCM segue da análise do algoritmo FCM. Portanto, a complexidade de espaço do algoritmo de agrupamento linearFCM é $O\left(N n+k N+n^{2}\right)$. Quando A é a matriz identidade a complexidade de espaço é $O(N n+k N)$.

\subsubsection{Algoritmos que Lidam com Atributos Categóricos}

Muitas aplicações práticas lidam com objetos descritos por atributos não numéricos ou não contínuos, tais como: categóricos ordinais, categóricos nominais, numéricos discre- 
tos, entre outros. Uma das maneiras de agrupar tais objetos consiste em construir uma matriz relacional dos dados (de acordo com alguma medida de (dis)similaridade) e então aplicar um algoritmo relacional (Seção 2.2.6). Entretanto, existem algoritmos que lidam naturalmente com tais objetos.

\subsubsection{FSCM: Fuzzy Symbolic c-Means}

De maneira similar ao algoritmo FCM, o algoritmo FSCM (Fuzzy Symbolic c-Means — em inglês) (El-Sonbaty \& Ismail, 1998) também procura minimizar (2.6). Porém, os protótipos dos grupos, $\mathbf{v}_{i}(i=1, \ldots, k)$, não são mais pontos médios no espaço. As distâncias, $D_{i j}(i=1, \ldots, k$ e $j=1, \ldots, N)$, entre os objetos $\mathbf{x}_{j}(j=1, \ldots, N)$ e os protótipos $\mathbf{v}_{i}(i=1, \ldots, k)$ também necessitam ser calculadas diferentemente. Dessa forma, os protótipos são definidos como:

$$
\mathbf{v}_{i}=\left[\mathbf{v}_{i 1}, \mathbf{v}_{i 2}, \ldots, \mathbf{v}_{i n}\right]
$$

em que $\mathbf{v}_{i p}(p=1, \ldots, n)$ agora são vetores que representam os $n$ atributos dos protótipos e são definidos como $(1 \leq i \leq k ; 1 \leq p \leq n)$ :

$$
\mathbf{v}_{i p}=\left[\left(A_{p, 1}, e_{i, p, 1}\right),\left(A_{p, 2}, e_{i, p, 2}\right), \ldots,\left(A_{p, n_{p}}, e_{i, p, n_{p}}\right)\right],
$$

em que $A_{p, l}\left(l=1, \ldots, n_{p}\right)$ representa o $l$-ésimo valor ${ }^{18}$ do $p$-ésimo atributo, $n_{p}$ é a quantidade de valores diferentes do $p$-ésimo atributo e $e_{i, p, l}\left(l=1, \ldots, n_{p}\right)$ representa a importância que o $i$-ésimo grupo dá para $A_{p, l}$ e é dada por $(1 \leq i \leq k ; 1 \leq p \leq n ; 1 \leq l \leq$ $\left.n_{p}\right)$ :

$$
e_{i, p, l}=\sum_{j=1}^{N}\left(u_{i j}\right)^{m} \phi_{j, p, l}
$$

$\phi_{j, p, l}=\left\{\begin{array}{ll}1, & \text { se o } p \text {-ésimo atributo do } j \text {-ésimo objeto contiver o l-ésimo valor. } \\ 0, & \text { caso contrário. }\end{array}\right.$,

A distância entre o protótipo $\mathbf{v}_{i}$ e o objeto $\mathbf{x}_{j}\left(D_{i j}\right)$ é dada por:

$$
D_{i j}=\left[\sum_{p=1}^{n} \sum_{l=1}^{n_{p}} d\left(x_{j p}, A_{p, l}\right) \cdot e_{i, p, l}\right]^{2}
$$

em que $x_{j p}$ é o valor do $p$-ésimo atributo do objeto $\mathbf{x}_{j}$ e $d\left(x_{j p}, A_{p, t}\right)$ é uma composição de distâncias de acordo com três aspectos:

$$
d\left(x_{j p}, A_{p, t}\right)=d_{p}\left(x_{j p}, A_{p, t}\right)+d_{s}\left(x_{j p}, A_{p, t}\right)+d_{c}\left(x_{j p}, A_{p, t}\right)
$$

que os autores (El-Sonbaty \& Ismail, 1998) referenciam como "devido à posição" $\left(d_{p}(\cdot)\right)$,

\footnotetext{
${ }^{18}$ Lembre-se que este algoritmo trabalha com objetos que contém atributos simbólicos. Então, "verde" é um valor do atributo "cor dos olhos", por exemplo.
} 
"devido à extensão" $\left(d_{s}(\cdot)\right)$ e "devido ao conteúdo" $\left(d_{c}(\cdot)\right)$. Tais distâncias são calculadas diferentemente dependendo se o atributo em questão ( $p$-ésimo atributo) é do tipo quantitativo ou qualitativo. Para atributos quantitativos, as distâncias são calculadas como:

$$
\begin{gathered}
d_{p}\left(x_{j p}, A_{p, t}\right)=\frac{\mid\left(\text { limite inferior de } x_{j p}\right)-\left(\text { limite inferior de } A_{p, t}\right) \mid}{(\text { tamanho do maior intervalo do } p \text {-ésimo atributo })}, \\
d_{s}\left(x_{j p}, A_{p, t}\right)=\frac{\mid\left(\text { tamanho de } x_{j p}\right)-\left(\text { tamanho de } A_{p, t}\right) \mid}{\left(\text { extensão de } x_{j p} \text { e } A_{p, t}\right)}, \\
d_{c}\left(x_{j p}, A_{p, t}\right)=\frac{\left(\text { tamanho de } x_{j p}\right)+\left(\text { tamanho de } A_{p, t}\right)}{\left(\text { extensão de } x_{j p} \text { e } A_{p, t}\right)} \\
-\frac{2 \cdot\left(\text { tamanho da interseção de } x_{j p} \text { e } A_{p, t}\right)}{\left(\text { extensão de } x_{j p} \text { e } A_{p, t}\right)}
\end{gathered}
$$

em que (tamanho do maior intervalo do $p$-ésimo atributo) é a dado pela diferença entre o maior e o menor valor do $p$-ésimo atributo considerando todos os objetos da base de dados e (extensão de $x_{j p}$ e $A_{p, t}$ ) é dado pelo menor intervalo contendo $x_{j p}$ e $A_{p, t}$ simultaneamente ${ }^{19}$. Para atributos quantitativos racionais (e.g. atributo contendo um único valor real), (limite inferior de $x_{j p}$ ) é definido como sendo o próprio valor de $x_{j p}$ e (tamanho de $x_{j p}$ ) é definido como zero e, consequentemente, a distância é calculada apenas com (2.70).

Para atributos qualitativos, as distâncias são calculadas como:

$$
d_{p}\left(x_{j p}, A_{p, t}\right)=0
$$

$d_{s}\left(x_{j p}, A_{p, t}\right)$ segundo $(2.71)$ e $d_{c}\left(x_{j p}, A_{p, t}\right)$ segundo (2.72).

Assim, o procedimento iterativo do algoritmo FSCM consiste em passos alternados derivados das condições necessárias para minimizar (2.6) e é descrito no Algoritmo 23.

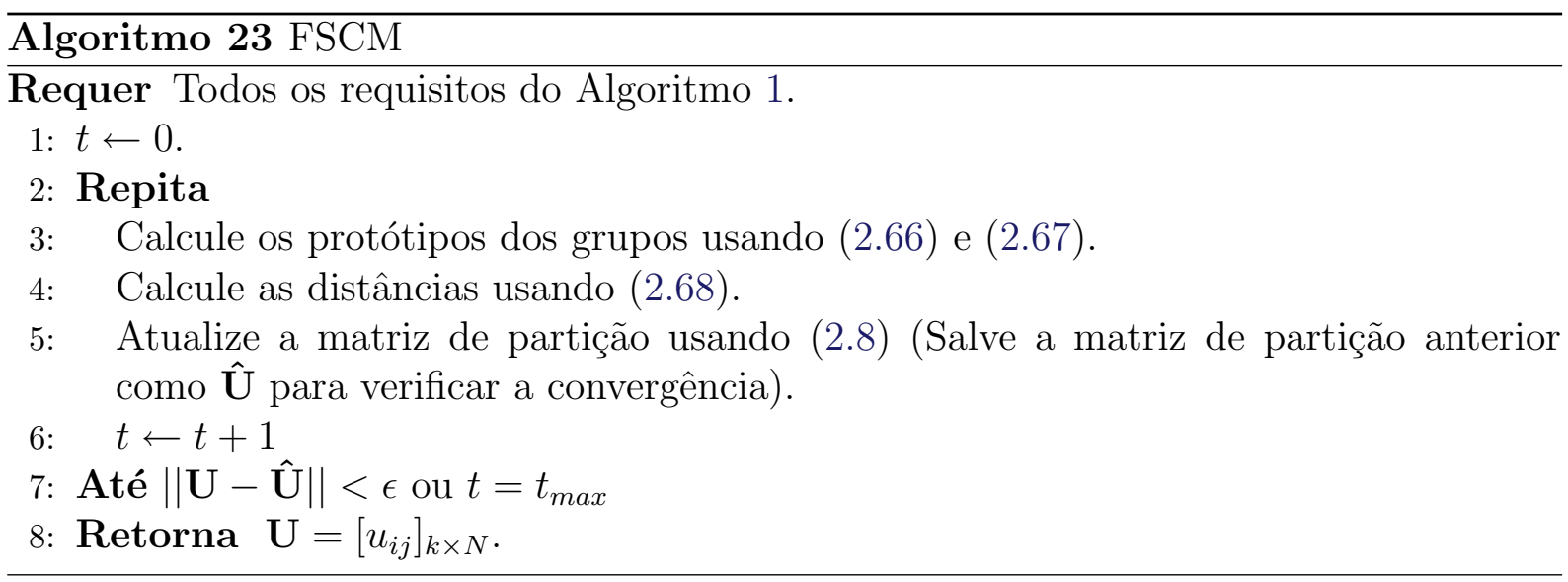

\footnotetext{
${ }^{19}$ Essas definições são usadas para atributos definidos por um intervalo, por exemplo, o atributo "recomendação de idade" em que um determinado objeto pode apresentar o valor [8-16] (recomendável dos 8 anos até os 16 anos).
} 
O cálculo dos protótipos em (2.66) e (2.67) requer $O\left(n_{p} k N n\right)$ operações. O cálculo das distâncias em (2.68) requer $O\left(n_{p} k N n\right)$ operações. A atualização das pertinências em (2.8) requer $O(k N)$ operações, usando o mesmo procedimento adotado por Kolen \& Hutcheson (2002). Portanto, a complexidade de tempo do algoritmo de agrupamento FSCM é $O\left(t\left(n_{p} k N n+n_{p} k N n+k N\right)\right) \rightarrow O\left(t n_{p} k N n\right)$.

\section{Análise de Complexidade - Espaço}

A base de dados $\mathbf{X}$ requer $O(N n)$ unidades de espaço. A matriz de partição requer $O(k N)$. Os protótipos requerem $O\left(n_{p} k n\right)$. As distâncias são calculadas e armazenadas em uma tabela à parte, o que requer $O(k N)$ unidades de espaço. Portanto, dado que $N>k$, a complexidade de espaço do algoritmo de agrupamento FSCM é $O\left(N n+k N+n_{p} k n+k N\right) \rightarrow$ $O\left(N n+k N+n_{p} k n\right)$.

\subsubsection{Análise de Complexidade - Resumo}

As complexidades de tempo e espaço dos algoritmos de agrupamento fuzzy revisados neste capítulo estão sumarizados nas Tabelas 2.10 e 2.11 .

\subsection{Outros Algoritmos}

Nas seções anteriores, foi apresentada uma revisão detalhada de vários algoritmos de agrupamento fuzzy de dados, incluindo suas análises de complexidade. Cada algoritmo apresenta uma característica diferente e foi proposto para determinado domínio. Embora tais algoritmos sejam bastante citados e utilizados na literatura, eles não são os únicos algoritmos propostos para a área de agrupamento fuzzy de dados. Nesta seção outros tipos de algoritmos são brevemente apresentados.

Na Seção 2.2.4 foram revisados alguns algoritmos que procuram por contornos de objetos, "cascas" ou extremidades. Existem vários outros algoritmos que também foram propostos com o mesmo objetivo. Os algoritmos AFCS (Adaptive Fuzzy c-Shells em inglês) (Dave \& Bhaswan, 1992) e FCES (Fuzzy c-Ellipsoidal Shells — em inglês) (Krishnapuram \& Frigui, 1996; Hoppner et al., 1999) procuram por contornos (bordas) em formatos elípticos. O algoritmo FCQS (Fuzzy c-Quadric Shells — em inglês) (Krishnapuram et al., 1992, 1993) é capaz de encontrar contornos de parábolas ou hipérboles, além de contornos de círculos e elipses. O algoritmo MFCQS (Modified Fuzzy c-Quadric Shells - em inglês) (Hoppner et al., 1999) apresenta uma melhoria na distância utilizada pelo algoritmo FCQS e, dessa forma, apresenta melhores resultados em alguns cenários específicos.

Foi comentado na Seção 2.2.5.3 que o algoritmo PCM (Seção 2.2.5.1) pode levar a uma solução na qual os protótipos estão todos em uma mesma posição (ou muito próximos), 


\begin{tabular}{lcc} 
Algoritmo & Complexidade de Tempo & Complexidade de Espaço \\
\hline FCM & $O\left(t k N n^{2}\right)$ & $O\left(N n+k N+n^{2}\right)$ \\
GK & $O\left(t k n^{3}+t k n^{2} N\right)$ & $O\left(N n+k N+k n^{2}\right)$ \\
GG & $O\left(t k n^{3}+t k n^{2} N\right)$ & $O\left(N n+k N+k n^{2}\right)$ \\
FCV & $O\left(t k n^{3}+t k n^{2} N r\right)$ & $O\left(N n+k N+k n^{2}\right)$ \\
FCE & $O\left(t k n^{3}+t k n^{2} N r\right)$ & $O\left(N n+k N+k n^{2}\right)$ \\
EFCM & $O\left(t k n^{3}+t k n^{2} N\right)$ & $O\left(N n+k N+k n^{2}\right)$ \\
PLFC & $O\left(t n^{2} N^{2}+t k^{2} N^{2}\right)$ & $O\left(N n+N^{2}\right)$ \\
FCS & $O\left(t k N n t_{N}\right)$ & $O(N n+k N)$ \\
FCSS & $O\left(t k N n^{2}+t k n^{3}\right)$ & $O\left(N n+k N+k n^{2}\right)$ \\
PCM & $O\left(t k N n^{2}\right)$ & $O\left(N n+k N+n^{2}\right)$ \\
PGK & $O\left(t k n^{3}+t k n^{2} N\right)$ & $O\left(N n+k N+k n^{2}\right)$ \\
FPCM & $O\left(t k N n^{2}\right)$ & $O\left(N n+k N+n^{2}\right)$ \\
PFCM & $O\left(t k N n^{2}\right)$ & \\
\hline \hline & & \\
\hline
\end{tabular}

Tabela 2.10: Complexidades de tempo e espaço para os algoritmos de agrupamento fuzzy: $t$ é o número de iterações, $k$ é o número de grupos, $n$ é o número de atributos, $N$ é o número de objetos, $t_{N}$ é o número de iterações do método de Newton e $r$ é a dimensão das variedades.

devido à independência entre os grupos existente nos algoritmos derivados do PCM (Barni et al., 1996; Timm et al., 2004). Adicionalmente aos algoritmos apresentados que foram desenvolvidos para evitar este problema (FPCM (Seção 2.2.5.3) e PFCM (Seção 2.2.5.4)), Timm et al. (2004) criaram uma versão modificada na qual foi adicionada à função objetivo do PCM original um fator de repulsão entre os protótipos dos grupos. Assim, foram desenvolvidos os algoritmos MPCM (Modified Possibilistic c-Means - em inglês) e MPGK (Modified Possibilistic GK - em inglês) que, dependendo de um parâmetro que mede o quão o fator de repulsão é importante, encontram partições possibilísticas evitando protótipos concêntricos.

Na Seção 2.2.8 foram apresentados alguns algoritmos que visam acelerar a execução do algoritmo FCM. Adicionalmente aos algoritmos apresentados, o algoritmo AFCM (Approximate Fuzzy c-Means - em inglês) (Cannon et al., 1986) transforma a matriz de dados 


\begin{tabular}{lcc} 
Algoritmo & Complexidade de Tempo & Complexidade de Espaço \\
\hline FCMdd & $O\left(t k N^{2}\right)$ & $O\left(N^{2}\right)$ \\
FCTMdd & $O\left(t k N+t N \log N+t k s^{2}\right)$ & $O\left(N^{2}\right)$ \\
RFCM & $O\left(t k N^{2}\right)$ & $O\left(N^{2}\right)$ \\
NERFCM & $O\left(t k N^{2}\right)$ & $O\left(N^{2}\right)$ \\
WDSFCM & $O\left(t k N^{2}\right)$ & $O(N n+k N)$ \\
PDSFCM & $O(t k N)$ & $O(N n+k N)$ \\
OCSFCM & $O\left(t k N^{2}\right)$ & $O(N n+k N)$ \\
NPSFCM & $O\left(t k N^{2}\right)$ & $O(N n+k N)$ \\
brFCM & $O\left(n N \log N+t k N^{0} n^{2}+k N\right)$ & $O(N n+k N)$ \\
mrFCM & $O\left(t k \Delta \% r^{2} n^{2}+t k N n^{2}\right)$ & $O\left(N n+k N+n^{2}\right)$ \\
linear-FCM & $O\left(t k N^{2}\right)$ & $O\left(N n+k N+n^{2}\right)$ \\
FSCM & $O\left(t n_{p} k N n\right)$ & $O\left(N n+k N+n_{p} k n\right)$ \\
\hline \hline
\end{tabular}

Tabela 2.11: Complexidades de tempo e espaço para os algoritmos de agrupamento fuzzy: $t$ é o número de iterações, $k$ é o número de grupos, $n$ é o número de atributos, $N$ é o número de objetos, $s$ é o número de objetos para o cálculo do medóide, $N^{0}$ é o número de objetos distintos da base de dados, $\Delta_{\%}$ é a porcentagem das amostras, $r$ é o número de amostras e $n_{p}$ é a quantidade de valores diferentes do $p$-ésimo atributo.

em uma matriz com dados inteiros e utiliza uma tabela para calcular as distâncias e as potências usadas pelo algoritmo. Dessa forma, espera-se que a quantidade de cálculos necessários para executar o AFCM seja menor em comparação com a execução do FCM. Mais além, os algoritmos AUP (Auto Update Prototypes - em inglês) e AUU (Auto Update $U$ - em inglês) (Kamel \& Selim, 1994) buscam mesclar os dois passos do algoritmo FCM (atualização dos protótipos e atualização da matriz de partição U) de maneira a atualizar os protótipos enquanto atualiza a matriz de partição (AUP) e atualizar a matriz de partição enquanto atualiza os protótipos (AUU). Ainda nesse contexto, Hung \& Yang (2001) usaram árvores-kd para otimizar a execução do algoritmo FCM.

Uma área de estudo recente que vem ganhando interesse de pesquisadores consiste em agrupamento de dados em fluxo contínuo de dados (clustering data-streams - em inglês). Nesses casos, os objetos são apresentados de maneira contínua ao algoritmo (possivelmente por tempo indeterminado) e o algoritmo de agrupamento deve agrupar tais 
objetos. Entretanto, devido à enorme quantidade de dados (podendo ser ilimitada nos casos de um fluxo contínuo ilimitado), os objetos não podem ser armazenados em memória e, consequentemente, os algoritmos descritos anteriormente não podem ser diretamente aplicados. Nesse contexto, os algoritmos propostos por Hore et al. (2007a,b, 2008) foram desenvolvidos de forma a receber uma determinada quantidade de objetos, executar o algoritmo de agrupamento FCM e sumarizar os dados utilizando os protótipos. Assim, apenas os protótipos são armazenados. Quando um novo conjunto de dados é recebido, o algoritmo reagrupa utilizando as informações dos protótipos armazenados e dos objetos recebidos. Diferentemente, no trabalho de Beringer \& Hüllermeier (2006) foi proposto um algoritmo para agrupar streams de dados. Nesse caso, cada stream é tratada como um objeto e streams que apresentam comportamento similares são agrupadas.

\subsection{Escolha do Algoritmo de Agrupamento}

Neste capítulo foram revisados vários algoritmos de agrupamento fuzzy de dados. Cada algoritmo apresenta características e complexidades computacionais diferentes, além de serem mais promissores em alguns cenários específicos de aplicação. Dada a grande quantidade de algoritmos existentes, o usuário precisa saber identificar quais são os algoritmos mais promissores em suas aplicações. Vários fatores precisam ser considerados para decidir que tipo de algoritmo utilizar (Tan et al., 2005):

- Tipo de agrupamento: O tipo de agrupamento é um fator muito importante, o qual diz respeito aos possíveis tipos de grupos que podem ser encontrados pelo algoritmo. Cada algoritmo de agrupamento busca encontrar grupos com formatos específicos (e.g. o algoritmo FCM (Seção 2.2.1) encontra grupos hiperesféricos, enquanto o algoritmo FCE (Seção 2.2.3.2) encontra grupos com formato de linhas, planos ou hiperplanos). O algoritmo escolhido deve procurar pelo mesmo tipo de grupos desejado.

- Característica da base: A característica da base de dados também é um fator importante. Se o usuário não dispõe dos atributos dos objetos, porém possui as dissimilaridades entre eles, então um algoritmo relacional (Seção 2.2.6) deve ser escolhido. Se os atributos não são numéricos ordinais, então pode-se utilizar um algoritmo que trabalhe com dados categóricos (Seção 2.2.9) ou pode-se calcular as dissimilaridades entre os objetos e utilizar um algoritmo relacional. Se a matriz de dados apresenta atributos faltantes, então pode ser necessário aplicar um préprocessamento aos dados para estimar os valores faltantes ou então deve-se utilizar um algoritmo que estime automaticamente esses valores (Seção 2.2.7).

- Dimensão do problema: A dimensão do problema também deve ser considerada. Se a aplicação apresenta uma quantidade muito grande de objetos, por exemplo, então técnicas que buscam executar os algoritmos mais rapidamente podem ser 
adotadas (Seção 2.2.8). Se a matriz de dados apresenta muitos atributos, então algoritmos que trabalham com matrizes de covariância podem apresentar deficiência numérica e custo computacional proibitivo para o cálculo da inversa dessas matrizes. Dessa forma, pode ser necessário aplicar um passo de pré-processamento dos dados para reduzir a dimensionalidade dos atributos (Tan et al., 2005).

- Complexidade computacional: A complexidade computacional de cada algoritmo também deve ser considerada. Por exemplo, alguns algoritmos podem ser mais robustos a outliers, como é o caso do algoritmo FCMdd (Seção 2.2.6.1), porém com um custo quadrático em relação ao número de objetos. Outros algoritmos apresentam complexidade linear em relação ao número de objetos, porém complexidade cúbica em relação ao número de atributos (e.g. GK — Seção 2.2.2.1). Para maiores detalhes das complexidades computacionais dos algoritmos, veja as Tabelas 2.10 e 2.11 na Seção 2.2.10.

- Especialista do domínio: Outro fator importante a ser considerado para a escolha do algoritmo diz respeito ao conhecimento do especialista do domínio. O especialista pode conhecer o problema em questão e disponibilizar informações importantes específicas do domínio e, dessa maneira, ajudar a guiar a escolha do algoritmo.

É importante destacar que nesta dissertação de mestrado foram estudados apenas algoritmos de agrupamento fuzzy de dados. Entretanto, vários outros tipos de algoritmos existentes na literatura foram desenvolvidos para outros cenários e também devem ser considerados durante o processo de escolha (Jain et al., 1999; Everitt et al., 2001; Tan et al., 2005; Xu \& Wunsch II, 2005; Gan et al., 2007; Xu \& Wunsch II, 2009).

Em resumo, a escolha de determinado algoritmo de agrupamento deve considerar vários aspectos. Não existe uma fórmula fechada para determinar o algoritmo de agrupamento mais promissor (Tan et al., 2005). Apesar disso, conhecer o funcionamento dos algoritmos de agrupamento disponíveis e considerar as questões mencionadas anteriormente, focando nas necessidades da aplicação, pode ajudar o usuário a determinar quais são os algoritmos mais promissores a serem utilizados.

\subsection{Validação de Agrupamento Fuzzy}

Os algoritmos de agrupamento apresentados anteriormente neste capítulo podem ser considerados não determinísticos se as matrizes de partição e/ou protótipos iniciais forem inicializados aleatoriamente (como usual), pois podem resultar em soluções (matriz de partição final) diferentes (e possivelmente de má qualidade) dependendo da inicialização. Adicionalmente, o número de grupos, $k$, que precisa ser fornecido a priori pelo usuário, é um parâmetro difícil de ser estimado na prática. Uma abordagem bem conhecida e utilizada para contornar esses problemas consiste em executar o algoritmo de agrupamento diversas vezes com matrizes de partição/protótipos inicias e número de grupos variados, 
então, escolher a partição mais adequada de acordo com algum critério. Dessa forma, é necessária uma medida para avaliar a qualidade das diversas soluções obtidas pelo algoritmo de agrupamento. Para essa tarefa é comum utilizar índices (ou critérios) de validade de agrupamento relativos, ou simplesmente índices de validação. Esses índices são capazes de quantificar a qualidade de soluções de agrupamento com base nos dados e de forma que diferentes soluções possam ser comparadas em termos relativos. Por isso são denominados relativos.

Nesta seção são apresentados alguns índices de validade fuzzy relativos existentes na literatura, que são utilizados para avaliar quantitativamente a qualidade das soluções obtidas por um algoritmo de agrupamento fuzzy.

\subsection{1 Índices Baseados Apenas na Matriz de Partição}

Alguns índices avaliam a qualidade das soluções utilizando as informações apenas da matriz de partição U. Em outras palavras, eles não utilizam informações sobre os atributos dos objetos ou (dis)similaridades entre eles. Alguns desses índices são revisados nesta seção.

\subsubsection{PC: Partition Coefficient}

O índice PC (Partition Coefficient — em inglês) (Bezdek, 1973, 1981) é uma medida de fuzzificação da matriz de partição $\mathbf{U}$, dada por:

$$
V_{P C}=\frac{1}{N} \sum_{i=1}^{k} \sum_{j=1}^{N}\left(u_{i j}\right)^{2} .
$$

Espera-se que boas partições, em geral, apresentem altos valores de $V_{P C}$, indicando que, para cada objeto, sua pertinência a um grupo específico é bem definida, i.e., para cada $j, u_{i j} \approx 1$ para um $i$ (consequentemente $u_{c j} \approx 0$ para $c \neq i$ ). Dessa maneira, o índice PC é um índice de maximização.

\section{Análise de Complexidade - Tempo}

O índice consiste em uma somatória de elementos da matriz de partição. Portanto, a complexidade de tempo do índice PC é $O(k N)$.

\section{Análise de Complexidade - Espaço}

O requisito necessário para o índice é somente a matriz de partição. Portanto, a complexidade de espaço é $O(k N)$.

\subsubsection{PE: Partition Entropy}

De maneira similar ao índice PC, o índice PE (Partition Entropy - em inglês) (Bezdek, 1975, 1981) mede a qualidade de uma dada partição analisando somente a matriz de 
partição U. Esse índice é baseado na entropia da matriz de partição, dada por:

$$
V_{P E}=-\frac{1}{N} \sum_{i=1}^{k} \sum_{j=1}^{N} u_{i j} \cdot \log _{a}\left(u_{i j}\right),
$$

em que $a$ é uma base logarítmica (e.g. $a=2$ ). Espera-se que boas partições apresentem, em geral, baixos valores de $V_{P E}$, indicando que a matriz de partição apresenta uma entropia baixa, i.e., para cada objeto, sua pertinência a um grupo específico é bem definida, i.e., para cada $j, u_{i j} \approx 1$ para um $i$ (consequentemente $u_{c j} \approx 0$ para $c \neq i$ ). Dessa forma, o índice PE é um índice de minimização.

\section{Análise de Complexidade - Tempo}

O índice consiste em uma somatória de elementos da matriz de partição. Portanto, a complexidade de tempo do índice PE é $O(k N)$.

\section{Análise de Complexidade - Espaço}

O requisito necessário para o índice é somente a matriz de partição. Portanto, a complexidade de espaço é $O(k N)$.

\subsubsection{MPC: Modified Partition Coefficient}

O índice MPC (Modified Partition Coefficient - em inglês) (Dave, 1996) tenta evitar a influência apresentada pelo índice $\mathrm{PC}$ em relação ao número de grupos $k$, uma vez que o índice PC apresenta valores no intervalo $[1 / k, 1]$, e é definido por:

$$
\begin{aligned}
V_{M P C} & =1-\frac{k}{k-1}\left(1-V_{P C}\right) \\
& =1-\frac{k}{k-1}\left(1-\frac{1}{N} \sum_{i=1}^{k} \sum_{j=1}^{N} u_{i j}^{2}\right) .
\end{aligned}
$$

Novamente, espera-se que boas partições apresentem, em geral, altos valores de $V_{M P C}$ e, dessa forma, MPC é um índice de maximização.

\section{Análise de Complexidade - Tempo}

O índice consiste em uma somatória de elementos da matriz de partição. Portanto, a complexidade de tempo do índice MPC é $O(k N)$.

\section{Análise de Complexidade - Espaço}

O requisito necessário para o índice é somente a matriz de partição. Portanto, a complexidade de espaço é $O(k N)$. 


\subsection{2 Índices Baseados na Matriz de Dados}

Índices que usam apenas a matriz de partição $\mathbf{U}$ (i.e., não usam informações dos atributos dos objetos e/ou informações de (dis)similaridades) podem levar a resultados indesejados (Wang \& Zhang, 2007), uma vez que as disposições dos objetos não são consideradas. Nesta seção são apresentados alguns índices de validade fuzzy que usam tanto a matriz de partição quanto a matriz de dados.

\subsubsection{XB: Xie-Beni}

O índice de XB (Xie-Beni) é definido como (Xie \& Beni, 1991; Pal \& Bezdek, 1995):

$$
V_{X B}=\frac{\sum_{i=1}^{k} \sum_{j=1}^{N}\left(u_{i j}\right)^{m}\left\|\mathbf{x}_{j}-\mathbf{v}_{i}\right\|^{2}}{N \min _{l \neq s}\left\|\mathbf{v}_{l}-\mathbf{v}_{s}\right\|^{2}} .
$$

O numerador de (2.77) é a distância intra-grupo total, que é equivalente à função objetivo $J$ em (2.6) com $D_{i j}$ definida como a distância Euclideana quadrada. A razão $J / N$ é um termo de normalização da partição. A distância mínima entre os protótipos no denominador de (2.77) é uma medida de separabilidade dos grupos. Dessa forma, espera-se que boas partições apresentem baixos valores de $V_{X B}$, i.e., XB é um índice de minimização.

\section{Análise de Complexidade - Tempo}

O índice contém uma somatória das distâncias entre cada objeto e cada protótipo, o que requer $O(k N n)$ operações, e entre pares de protótipos, o que requer $O\left(k^{2} n\right)$. Dado que $N>k$, a complexidade de tempo do índice XB é $O\left(k N n+k^{2} n\right) \rightarrow O(k N n)$.

\section{Análise de Complexidade - Espaço}

Esse índice requer a base de dados, a matriz de partição e os protótipos dos grupos. Portanto, a complexidade de espaço do índice XB é $O(N n+k N+k n) \rightarrow O(N n+k N)$.

\subsubsection{FSS: Fuzzy Simplified Silhouette}

O índice FSS (Fuzzy Simplified Silhouette — em inglês) (Campello \& Hruschka, 2006) é a versão fuzzy do índice SS (Simplified Silhouette — em inglês) (para partições rígidas) (Hruschka et al., 2006), e é definida como:

$$
V_{F S S}=\frac{\sum_{j=1}^{N}\left(u_{p j}-u_{q j}\right)^{\alpha} s_{j}}{\sum_{j=1}^{N}\left(u_{p j}-u_{q j}\right)^{\alpha}}
$$

em que $u_{p j}$ e $u_{q j}$ são respectivamente o primeiro e o segundo maior elemento da $j$-ésima coluna de matriz de partição, $\alpha \geq 0$ é um coeficiente de peso definido pelo usuário e $s_{j}$ é 
a silhueta individual do objeto $\mathbf{x}_{j}$, definido como:

$$
s_{j}=\frac{b_{p j}-a_{p j}}{\max \left\{a_{p j}, b_{p j}\right\}},
$$

em que $a_{p j}$ é a distância entre o objeto $\mathbf{x}_{j}$ e o protótipo mais próximo $\mathbf{v}_{p}$ (correspondendo ao grupo pelo qual $\mathbf{x}_{j}$ apresenta o maior valor de pertinência) e $b_{p j}$ é a distância entre o objeto $\mathbf{x}_{j}$ e o segundo protótipo mais próximo. É esperado que boas partições apresentem valores altos de $s_{j}$ (valores altos de $b_{p j}$ e baixos de $a_{p j}$ ) e, como consequência, altos valores de $V_{F S S}$ em (2.78). Dessa forma, FSS é um índice de maximização.

\section{Análise de Complexidade - Tempo}

O cálculo em (2.78) requer $O(k N)$ (incluindo o cálculo de $u_{p j}$ e $u_{q j}$ ). (2.79) pode ser calculada em $O(k N n)$, dado que consiste de distâncias entre objetos e protótipos. Portanto, a complexidade de tempo do índice FSS é $O(k N n)$.

\section{Análise de Complexidade - Espaço}

Esse índice requer a base de dados, a matriz de partição e os protótipos dos grupos. Portanto, a complexidade de espaço do índice FSS é $O(N n+k N+k n) \rightarrow O(N n+k N)$.

\subsubsection{K: Kwon}

O índice K (Kwon, 1998) é uma modificação do índice XB, para eliminar a tendência em função de $k$, e é dado por:

$$
V_{K}=\frac{\sum_{i=1}^{k} \sum_{j=1}^{N}\left(u_{i j}\right)^{2}\left\|\mathbf{x}_{j}-\mathbf{v}_{i}\right\|^{2}+\frac{1}{k} \sum_{i=1}^{k}\left\|\mathbf{v}_{i}-\overline{\mathbf{x}}\right\|^{2}}{\min _{l \neq s}\left\|\mathbf{v}_{l}-\mathbf{v}_{s}\right\|^{2}}
$$

em que

$$
\overline{\mathbf{x}}=\frac{1}{N} \sum_{j=1}^{N} \mathbf{x}_{j}
$$

é a média geral da base de dados. O primeiro termo do numerador em (2.80) mede a similaridade intra-grupo. O segundo é um fator que tenta contrabalancear a tendência de decrescimento em função de $k$. O denominador em (2.80) mede a distância inter-grupo. Dessa forma, espera-se que valores baixos de $V_{K}$ indiquem boas partições, i.e., K é um índice de minimização.

\section{Análise de Complexidade - Tempo}

O índice contém uma somatória de distâncias entre cada objeto e cada protótipo, o que requer $O(k N n)$ operações. Contém também uma somatória entre pares de protótipos, o que requer $O\left(k^{2} n\right)$ e entre os protótipos e a média geral, o que requer $O(k n)$. O cálculo 
da média geral em (2.81) requer $O(N n)$. Portanto, a complexidade de tempo do índice $\mathrm{K}$ é $O\left(k N n+k^{2} n+k n+N n\right) \rightarrow O\left(k N n+k^{2} n\right) \rightarrow O(k N n)$.

\section{Análise de Complexidade - Espaço}

O índice requer a base de dados, a matriz de partição, os protótipos dos grupos e a média geral. Portanto, a complexidade de espaço do índice $\mathrm{K}$ é $O(N n+k N+k n+n) \rightarrow$ $O(N n+k N+k n) \rightarrow O(N n+k N)$.

\subsubsection{TSS: Tang-Sun-Sun}

Com a mesma ideia do índice K, o índice TSS (Tang-Sun-Sun) (Tang et al., 2005) modifica o índice XB para eliminar a tendência decrescente em função de $k$ e é dado por:

$$
V_{T S S}=\frac{\sum_{i=1}^{k} \sum_{j=1}^{N}\left(u_{i j}\right)^{2}\left\|\mathbf{x}_{j}-\mathbf{v}_{i}\right\|^{2}+\frac{1}{k(k-1)} \sum_{i=1}^{k} \sum_{\substack{l=1 \\ l \neq i}}^{k}\left\|\mathbf{v}_{i}-\mathbf{v}_{l}\right\|^{2}}{\min _{l \neq s}\left\|\mathbf{v}_{l}-\mathbf{v}_{s}\right\|^{2}+1 / k} .
$$

Novamente, o primeiro termo do numerador em (2.82) mede a similaridade intragrupo. O segundo é um fator que tenta contrabalancear o decrescimento em função de $k$. O denominador em (2.82) mede a distância inter-grupo. Dessa forma, espera-se que valores pequenos de $V_{T S S}$ indiquem boas partições, i.e., TSS é um índice de minimização.

\section{Análise de Complexidade - Tempo}

O índice contém uma somatória das distâncias entre cada objeto e cada protótipo, o que requer $O(k N n)$ e entre pares de protótipos, o que requer $O\left(k^{2} n\right)$. Portanto, a complexidade de tempo do índice TSS é $O\left(k N n+k^{2} n\right) \rightarrow O(k N n)$.

\section{Análise de Complexidade - Espaço}

O índice requer a base de dados, a matriz de partição e os protótipos dos grupos. Portanto, a complexidade de espaço do índice TSS é $O(N n+k N+k n) \rightarrow O(N n+k N)$.

\subsubsection{FS: Fukuyama-Sugeno}

O índice FS (Fukuyama e Sugeno) (Pal \& Bezdek, 1995; Fukuyama \& Sugeno, 1989) é definido como:

$$
V_{F S}=\sum_{i=1}^{k} \sum_{j=1}^{N}\left(u_{i j}\right)^{m}\left\|\mathbf{x}_{j}-\mathbf{v}_{i}\right\|^{2}-\sum_{i=1}^{k} \sum_{j=1}^{N}\left(u_{i j}\right)^{m}\left\|\mathbf{v}_{i}-\overline{\mathbf{x}}\right\|^{2},
$$

em que $\overline{\mathbf{x}}$ é dado por (2.81). O primeiro termo em (2.83) mede a dissimilaridade intragrupo. O segundo $\left(\sum_{i=1}^{k} \sum_{j=1}^{N}\left(u_{i j}\right)^{m}\left\|\mathbf{v}_{i}-\overline{\mathbf{x}}\right\|^{2}\right)$ tende a crescer conforme o número de grupos cresce. Em contrapartida, este termo também tende a decrescer conforme o grau de

sobreposição (fuzzificação de $\mathbf{U}$ ) entre os grupos cresce. Fukuyama e Sugeno argumentam 
que boas partições são identificadas por baixos valores de $V_{F S}$. Portanto, FS é um índice de minimização.

\section{Análise de Complexidade - Tempo}

O índice contém uma somatória de distâncias entre cada objeto e cada protótipo, o que requer $O(k N n)$ operações, e entre os protótipos e a média geral, o que requer $O(k n)$. O cálculo da média geral em (2.81) requer $O(N n)$. Dessa forma, a complexidade de tempo do índice FS é $O(k N n+k n+N n) \rightarrow O(k N n)$.

\section{Análise de Complexidade - Espaço}

O índice requer a base de dados, a matriz de partição, os protótipos dos grupos e a média geral dos dados. Portanto, a complexidade de espaço do índice FS é $O(N n+k N+$ $k n+n) \rightarrow O(N n+k N+k n) \rightarrow O(N n+k N)$.

\subsubsection{FHV: Fuzzy Hypervolume}

O índice FHV (Fuzzy Hypervolume - em inglês) (Gath \& Geva, 1989) é baseado no conceito de hipervolume e densidade, sendo definido como:

$$
V_{F H V}=\sum_{i=1}^{k}\left[\operatorname{det}\left(\mathbf{F}_{i}\right)\right]^{1 / 2},
$$

em que $\mathbf{F}_{i}$ é dado por $(2.13)^{20}$. Dado que os autovalores de $\mathbf{F}_{i}$ são relacionados com as variâncias do $i$-ésimo grupo fuzzy, o determinante em (2.84), dado pelo produto de todos os autovalores de $\mathbf{F}_{i}$, provê uma medida de dispersão (hipervolume) do grupo. Dessa forma, espera-se que boas partições apresentem baixos valores de $V_{F H V}$ e, portanto, FHV é um índice de minimização.

\section{Análise de Complexidade - Tempo}

Considerando que as matrizes de covariância fuzzy, $\mathbf{F}_{i}$, são fornecidas ${ }^{21},(2.84)$ pode ser calculado em $O\left(k n^{3}\right)$. Portanto, a complexidade de tempo do índice FHV é $O\left(k n^{3}\right)$. Caso contrário, se $\mathbf{F}_{i}$ não são fornecidas, um passo adicional para calcular (2.13) é necessário, o que requer $O\left(k n^{3}+k N n^{2}\right)$. Portanto, a complexidade de tempo do índice FHV é $O\left(k n^{3}+k N n^{2}\right)$ quando $\mathbf{F}_{i}$ não são fornecidas.

\section{Análise de Complexidade - Espaço}

O índice requer somente as matrizes de covariância fuzzy. Portanto a complexidade de espaço do índice FHV é $O\left(k n^{2}\right)$.

\footnotetext{
${ }^{20}$ Alguns altores usam $\mathbf{F}_{i}$ em (2.13) com $m=1$ (Bouguessa et al., 2006; Campello \& Hruschka, 2006) enquanto outros usam com valores diferentes de $m$ (Halkidi et al., 2001; Wang \& Zhang, 2007).

${ }^{21}$ Por exemplo quando o índice é usado para avaliar partições de um dos seguintes algoritmos: GK (Seção 2.2.2.1), GG (Seção 2.2.2.2), FCV (Seção 2.2.3.1) e FCE (Seção 2.2.3.2).
} 


\subsubsection{APD: Average Partition Density}

Baseado na mesma ideia do índice FHV o índice APD (Average Partition Density em inglês) (Gath \& Geva, 1989) é definido como:

$$
V_{A P D}=\frac{1}{k} \sum_{i=1}^{k} \frac{S_{i}}{\left[\operatorname{det}\left(\mathbf{F}_{i}\right)\right]^{1 / 2}}
$$

em que

$$
S_{i}=\sum_{\mathbf{x}_{j} \in \mathbf{R}_{i}} u_{i j}
$$

e $\mathbf{R}_{i}$ é um conjunto de objetos que estão em uma região pré-estabelecida em torno do protótipo $\mathbf{v}_{i}$, i.e., os objetos que apresentam $\left(\mathbf{x}_{j}-\mathbf{v}_{i}\right)^{T} \mathbf{F}_{i}^{-1}\left(\mathbf{x}_{j}-\mathbf{v}_{i}\right)<1$. Dado que grupos compactos apresentam valores baixos de $\left[\operatorname{det}\left(\mathbf{F}_{i}\right)\right]^{1 / 2}$ e valores altos de $S_{i}$ em (2.85), espera-se que boas partições apresentem valores altos de $V_{A P D}$ e, portanto, APD é um índice de maximização.

\section{Análise de Complexidade - Tempo}

Considerando que as matrizes de covariância fuzzy, $\mathbf{F}_{i}$, são fornecidas e dado que (2.86) requer $O\left(k N n^{3}\right)$ para $i=1, \ldots, k,(2.85)$ pode ser calculado em $O\left(k n^{3}\right)$. Portanto, a complexidade de tempo do índice APD é $O\left(k n^{3}+k N n^{3}\right) \rightarrow O\left(k N n^{3}\right)$. Note que mesmo se $\mathbf{F}_{i}$ não forem fornecidas, o que requer um passo adicional para calcular (2.13), requerendo $O\left(k n^{3}+k N n^{2}\right)$, a complexidade de tempo do índice APD também é dada por $O\left(k N n^{3}+k n^{3}+k N n^{2}\right) \rightarrow O\left(k N n^{3}\right)$.

\section{Análise de Complexidade - Espaço}

O índice requer as matrizes de covariância fuzzy e armazena $S_{i}$ em (2.86) para cada grupo. Portanto, a complexidade de espaço do índice APD é $O\left(k n^{2}+k\right) \rightarrow O\left(k n^{2}\right)$.

\subsubsection{PD: Partition Density}

Baseado no índice APD, o índice PD (Partition Density — em inglês) (Gath \& Geva, 1989) é definido como:

$$
V_{P D}=\frac{\sum_{i=1}^{k} S_{i}}{V_{F H V}},
$$

em que $S_{i}$ é dado por (2.86) e $V_{F H V}$ é dado por (2.84). Como consequência, esperase que boas partições apresentem valores altos de $V_{P D}$ e, portanto, PD é um índice de maximização. 
O índice FHV requer $O\left(k n^{3}+k N n^{2}\right)$ e $(2.86)$ requer $O\left(k N n^{3}\right)$ para $i=1, \ldots, k$. Portanto, a complexidade de tempo do índice PD é $O\left(k n^{3}+k N n^{3}\right) \rightarrow O\left(k N n^{3}\right)$.

\section{Análise de Complexidade - Espaço}

O índice requer as matrizes de covariância fuzzy e armazena $S_{i}$ em (2.86) para cada grupo. Portanto, a complexidade de espaço do índice PD é $O\left(k n^{2}+k\right) \rightarrow O\left(k n^{2}\right)$.

\subsubsection{SCG}

O índice SCG (Bouguessa \& Wang, 2004) é definido como:

$$
V_{S C G}=\frac{S e p}{G C o m p}
$$

em que GComp é uma medida de compacidade definida por:

$$
\text { GComp }=\sum_{i=1}^{k} \operatorname{trace}\left(\mathbf{F}_{i}\right)
$$

com $\mathbf{F}_{i}$ dado por (2.13) e Sep é a separabilidade definida como:

$$
S e p=\operatorname{trace}\left(\mathbf{S}_{B}\right)
$$

$\mathbf{S}_{B}$ é a matriz definida por:

$$
\mathbf{S}_{B}=\sum_{i=1}^{k} \sum_{j=1}^{N}\left(u_{i j}\right)^{m}\left(\mathbf{v}_{i}-\overline{\mathbf{x}}\right)\left(\mathbf{v}_{i}-\overline{\mathbf{x}}\right)^{T}
$$

em que $\overline{\mathbf{x}}$ é dado por (2.81). É esperado que boas partições apresentem valores altos de Sep e valores baixos de GComp (grupos compactos e bem separados). Assim, espera-se que valores altos de $V_{S C G}$ indiquem boas partições e, consequentemente, SCG é um índice de maximização.

\section{Análise de Complexidade - Tempo}

Considerando que as matrizes de covariância fuzzy, $\mathbf{F}_{i}$, são fornecidas, (2.89) pode ser calculado em $O(k n)$ operações. O cálculo de Sep em (2.90) e (2.91) requer $O(n)$ e $O\left(k N n^{2}+N n\right)$ (incluindo o cálculo da média geral), respectivamente. Portanto, a complexidade de tempo do índice SCG é $O\left(k n+n+k N n^{2}+N n\right) \rightarrow O\left(k N n^{2}\right)$. Caso $\mathbf{F}_{i}$ não sejam fornecidas, um passo adicional é necessário para o cálculo de (2.13), o que requer $O\left(k n^{3}+k N n^{2}\right)$. Dessa forma, a complexidade de tempo do índice SCG é $O\left(k n^{3}+k N n^{2}\right)$ quando $\mathbf{F}_{i}$ não são fornecidas. 
Análise de Complexidade - Espaço

O índice requer a base de dados, os protótipos dos grupos e as matrizes de covariância fuzzy, e armazena $\mathbf{S}_{B}$ e a média geral. Portanto, a complexidade de espaço do índice SCG é $O\left(N n+k n+k N+n^{2}+n\right) \rightarrow O\left(N n+k n+k N+n^{2}\right) \rightarrow O\left(N n+k N+n^{2}\right)$.

\subsubsection{PBMF}

O índice PBMF (Pakhira et al., 2004) é dado por:

$$
V_{P B M F}=\left(\frac{1}{k} \cdot \frac{E_{1}}{J_{m}} \cdot D_{c}\right)^{2},
$$

em que $E_{1}=\sum_{j=1}^{N}\left\|\mathbf{x}_{j}-\overline{\mathbf{x}}\right\|$ é uma constante que depende da base de dados e é usada para evitar valores muito pequenos de $V_{P B M F}, D_{c}=\max _{i, j=1}^{k}\left\|\mathbf{v}_{i}-\mathbf{v}_{j}\right\|$ mede a distância máxima entre os protótipos dos grupos e $J_{m}=\sum_{i=1}^{k} \sum_{j=1}^{N}\left(u_{i j}\right)^{m}\left\|\mathbf{x}_{j}-\mathbf{v}_{i}\right\|$ mede a dissimilaridade intra-grupo. É esperado que boas partições apresentem valores baixos de $J_{m}$ e altos de $D_{c}$. Assim, espera-se que valores altos de $V_{P B M F}$ indiquem boas partições fuzzy e, consequentemente, PBMF é um índice de maximização.

\section{Análise de Complexidade - Tempo}

O cálculo de $E_{1}$ requer $O(N n)$ operações (incluindo o cálculo da média geral). Calcular $J_{m}$ requer $O(k N n)$. O cálculo de $D_{c}$ requer $O\left(k^{2} n\right)$. Portanto, a complexidade de tempo do índice PBMF é $O\left(N n+k N n+k^{2} n\right) \rightarrow O\left(k N n+k^{2} n\right) \rightarrow O(k N n)$.

\section{Análise de Complexidade - Espaço}

O índice requer a base de dados, a matriz de partição, os protótipos dos grupos e armazena a média geral. Portanto, a complexidade de espaço do índice PBMF é $O(N n+$ $k N+k n+n) \rightarrow O(N n+k N+k n) \rightarrow O(N n+k N)$.

\subsubsection{Análise de Complexidade - Resumo}

As análises de complexidade de tempo e espaço de cada índice de validade de agrupamento estão sumarizadas na Tabela 2.12 .

\subsection{Estimação do Número de Grupos}

Um dos parâmetros necessários para a execução dos algoritmos apresentados na Seção 2.2 é o número, $k$, de grupos. Entretanto, o número mais natural de grupos em uma base de dados geralmente não é conhecido a priori pelo usuário. Na prática, diversas abordagens para determinar o número mais apropriado de grupos podem ser utilizadas (Babuska, 1998; Hoppner et al., 1999). Uma abordagem bem conhecida e utilizada na 


\begin{tabular}{lcc} 
Índice & Complexidade de Tempo & Complexidade de Espaço \\
\hline PC & $O(k N)$ & $O(k N)$ \\
PE & $O(k N)$ & $O(k N)$ \\
MPC & $O(k N)$ & $O(k N)$ \\
XB & $O(k N n)$ & $O(N n+k N)$ \\
FSS & $O(k N n)$ & $O(N n+k n)$ \\
K & $O(k N n)$ & $O(N n+k N)$ \\
TSS & $O(k N n)$ & $O(N n+k N)$ \\
FS & $O(k N n)$ & $O(N n+k N)$ \\
FHV & $O\left(k n^{3}+k N n^{2}\right)$ & $O\left(k n^{2}\right)$ \\
APD & $O\left(k N n^{3}\right)$ & $O\left(k n^{2}\right)$ \\
PD & $O\left(k N n^{3}\right)$ & $O\left(k n^{2}\right)$ \\
SCG & $O\left(k n^{3}+k N n^{2}\right)$ & $O\left(N n+k N+n^{2}\right)$ \\
PBMF & $O(k N n)$ & $O(N n+k N)$ \\
\hline
\end{tabular}

Tabela 2.12: Complexidade de tempo e espaço para os índices de validade de agrupamento fuzzy: $k$ é o número de grupos, $n$ é o número de atributos e $N$ é o número de objetos.

literatura consiste em executar o algoritmo de agrupamento várias vezes com número variado de grupos, obtendo portanto várias partições dos dados com qualidade e número de grupos diferente, e escolher dentre todas as partições aquela que apresenta o melhor resultado de acordo com um determinado critério (e.g. índices fuzzy relativos apresentados na Seção 2.5).

Tal abordagem, denotada por OMR-A (Ordered Multiple Runs of $\mathcal{A}$ - em inglês), consiste no procedimento pelo qual o algoritmo de agrupamento desejado, $\mathcal{A}$ (e.g. OMRFCM para o algoritmo FCM), executa $M$ vezes (a partir de inicializações aleatórias distintas dos protótipos ou matrizes de partição) para cada número de grupos $k$ variando de um dado $k_{\min }$ (usualmente 2 ) até um dado $k_{\max }$. No final desse procedimento existirão $M \cdot\left(k_{\max }-k_{\min }+1\right)$ partições que serão avaliadas de acordo com determinado índice de 
validade, $\mathcal{I}$. Este procedimento está detalhado no Algoritmo 24.

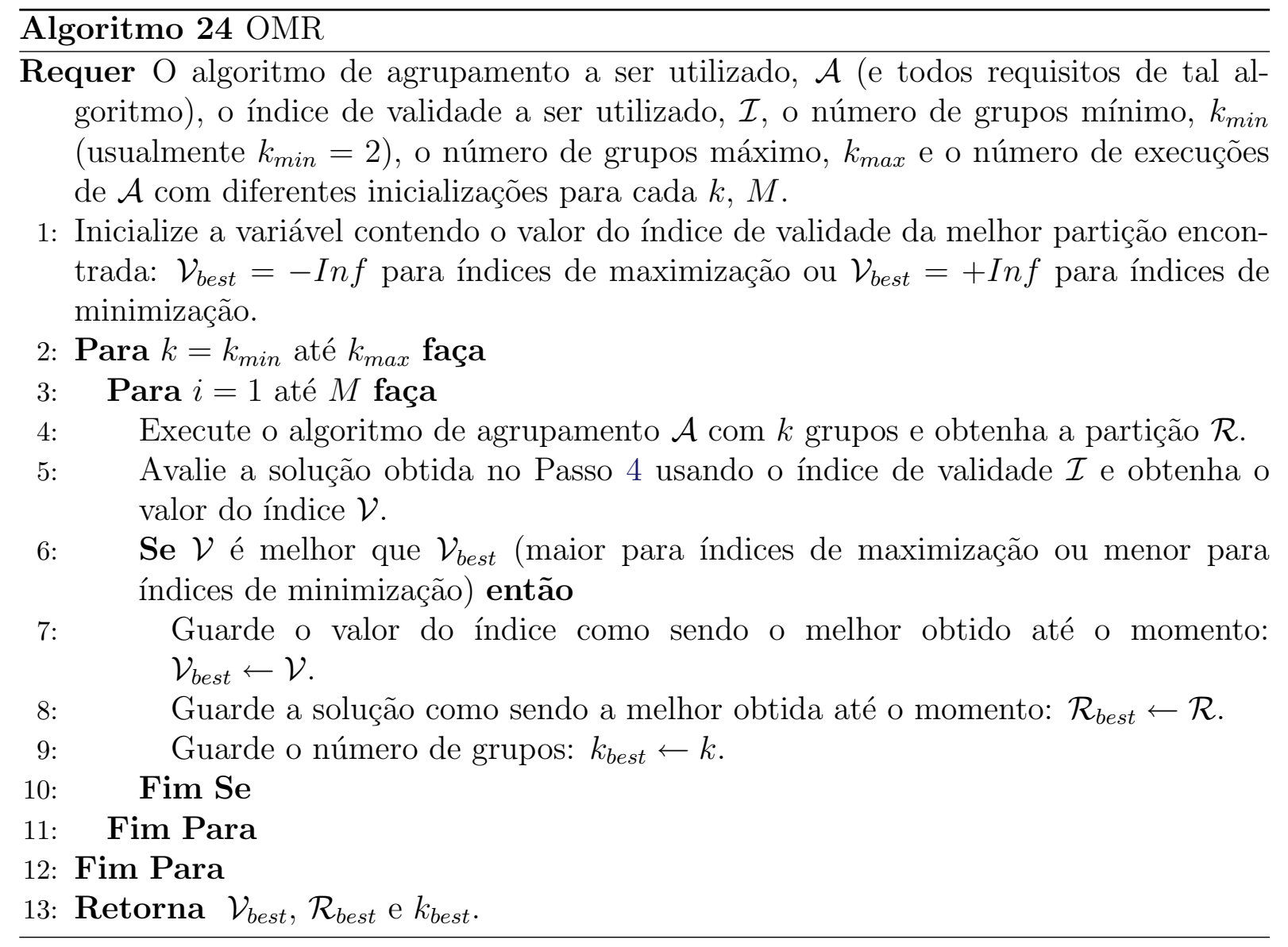

É importante destacar que o índice de validade, $\mathcal{I}$, escolhido para o procedimento OMR- $\mathcal{A}$, deve estar em conformidade com o algoritmo utilizado, $\mathcal{A}$. Alguns autores utilizam distâncias modificadas (e.g. distância de Mahalanobis usando as matrizes de covariância resultantes do algoritmo) dos índices originais para trabalhar com determinados algoritmos (Campello \& Hruschka, 2006). Outros utilizam a própria função objetivo do algoritmo como um índice de validade e então fazem uma análise dos valores obtidos dentre todas as partições para encontrar a melhor solução (Yoshinari et al., 1993; Nikhil et al., 1997), e.g., plotando os valores da função objetivo em relação ao número de grupos e então encontrando um joelho (ou cotovelo) no gráfico ou convertendo os valores da função objetivo em relação ao número de grupos em um valor relativo que pode ser usado como índice de validação (Vendramin et al., 2010). 


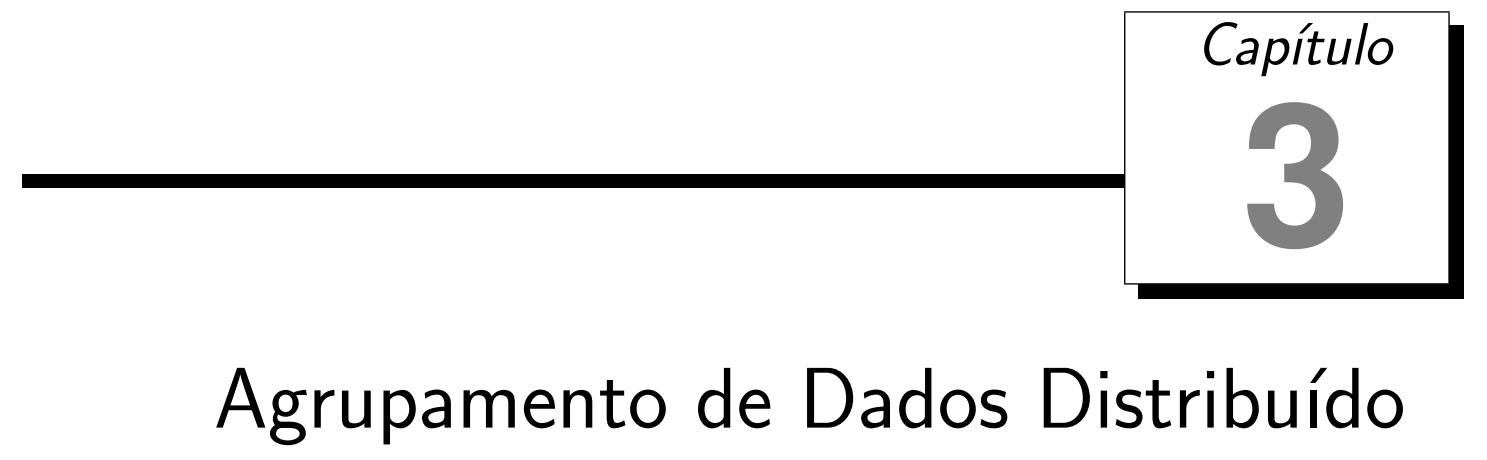

Atualmente, ambientes distribuídos tais como a internet, intranets e ambiente de processamento paralelo estão mudando vários aspéctos da computação, sendo adotados em diversas áreas de pesquisa e em ambientes práticos de aplicação (Attiya \& Welch, 2004; Cristea et al., 2010). Essa tendência também pode ser observada nas áreas de descoberta de conhecimento, aprendizado de máquina e mineração de dados, incluindo agrupamento de dados (Park et al., 2001; Park \& Kargupta, 2002; Tsoumakas et al., 2004; Datta et al., 2006). Mais especificamente, tal tendência deu origem a áreas de estudo denominadas Descoberta de Conhecimento Distribuído (Distributed Knowledge Discovery in Databases — em inglês) (Zaki \& Pan, 2002; Congiusta et al., 2007), Mineração de Dados Paralela e Distribuída (Parallel and Distributed Data Mining — em inglês) (Johnson \& Kargupta, 2000; Kargupta et al., 2001; Park \& Kargupta, 2002; Zaki \& Pan, 2002; Klusch et al., 2003; Zhang et al., 2004; da Silva et al., 2005; Merugu \& Ghosh, 2005; da Silva \& Klusch, 2007; Congiusta et al., 2007) e Agrupamento de Dados Paralelo e Distribuído (Parallel and Distributed Data Clustering — em inglês) (da Silva \& Klusch, 2006; Merugu \& Ghosh, 2005; Klusch et al., 2003).

Embora exista uma certa relação entre as áreas de Mineração de Dados Paralela e Mineração de Dados Distribuída (Freitas \& Lavington, 1997; Zaki, 2000), estes termos são geralmente usados para indicar algoritmos com diferentes características. O termo "paralelo" usualmente se refere a algoritmos aplicados a sistemas fortemente acoplados, enquanto o termo "distribuído" usualmente se refere a algoritmos aplicados a sistemas fracamente acoplados. Em outras palavras, algoritmos paralelos são geralmente usados em arquiteturas com múltiplos processadores em que os dados estão disponíveis em uma memória compartilhada, enquanto algoritmos distribuídos geralmente assumem que os dados estão em uma memória distribuída (Tanenbaum, 2001, 2002). Tipicamente, mineração de dados paralela se concentra em aumentar o desempenho computacional, enquanto minera- 
ção de dados distribuída destina-se a solucionar problemas em que não há formas factíveis de centralizar a base de dados. (Zaki, 1999). Neste capítulo, discutimos e desenvolvemos um framework para lidar tanto com cenários paralelos como com cenários distribuídos.

Quando os dados estão naturalmente distribuídos em diferentes sítios de dados, o leitor pode se perguntar por que os dados (distribuídos) não são centralizados antes de executar o algoritmo desejado. Note que a centralização dos dados pode não ser viável devido à grande quantidade de dados e, consequentemente, a uma demanda muito grande na transferência, armazenamento e tempo de processamento desses dados. Nesses casos, a centralização dos dados não é possível devido a limitações computacionais, tais como capacidade de memória ou tempo de processamento. Em outros casos, existe uma necessidade de preservar a confidencialidade dos dados. Em resumo, cenários distribuídos podem envolver limitações tais como: (Kargupta et al., 2001; Merugu \& Ghosh, 2005; Pedrycz, 2005; da Silva \& Klusch, 2006; Pedrycz, 2007; da Silva \& Klusch, 2007):

Privacidade: Os dados que estão armazenados em diferentes sítios de dados podem ser confidenciais. As informações dos dados não devem ser conhecidas por outros sítios de dados devido à confidencialidade.

Transferência: A transmissão de uma base de dados muito grande pode exigir uma largura de banda muita alta para ocorrer em um tempo aceitável.

Segurança: A transferência de dados entre os sítios requer procedimentos de segurança para que esses dados não sejam interceptados durante a comunicação.

Armazenamento: O custo de armazenar todos os dados em um único local físico pode ser muito elevado dependendo do tamanho da base de dados.

Processamento: O tempo de processamento de todos os dados em um único processador pode ser proibitivo.

Para contornar tais restrições, os dados distribuídos podem ser tratados separadamente. Tome como exemplo a Figura 3.1, na qual os dados estão distribuídos em dois sítios de dados (SD1 e SD2). Na Figura 3.1a, os dados são centralizados e o algoritmo de agrupamento é executado em um nó (computador) central. Na Figura 3.1b, por sua vez, o algoritmo de agrupamento distribuído é executado com os dados ainda em SD1 e SD2, e a mesma estrutura encontrada na Figura 3.1a é encontrada novamente, sem a necessidade da centralização dos dados ${ }^{1}$.

Duas formas de distribuição entre os sítios de dados são comumente aceitas: a distribuição horizontalmente particionada e a verticalmente particionada (Merugu \& Ghosh, 2005; da Silva et al., 2005). No primeiro caso, os sítios de dados contêm diferentes objetos relativos a um mesmo espaço de atributos. Já no segundo caso, os sítios de dados possuem

\footnotetext{
${ }^{1}$ Note que o cenário distribuído ilustrado na Figura $3.1 b$ pode ser criado artificialmente em vários processadores para obter melhor desempenho, o que nesse caso caracteriza um cenário paralelo.
} 


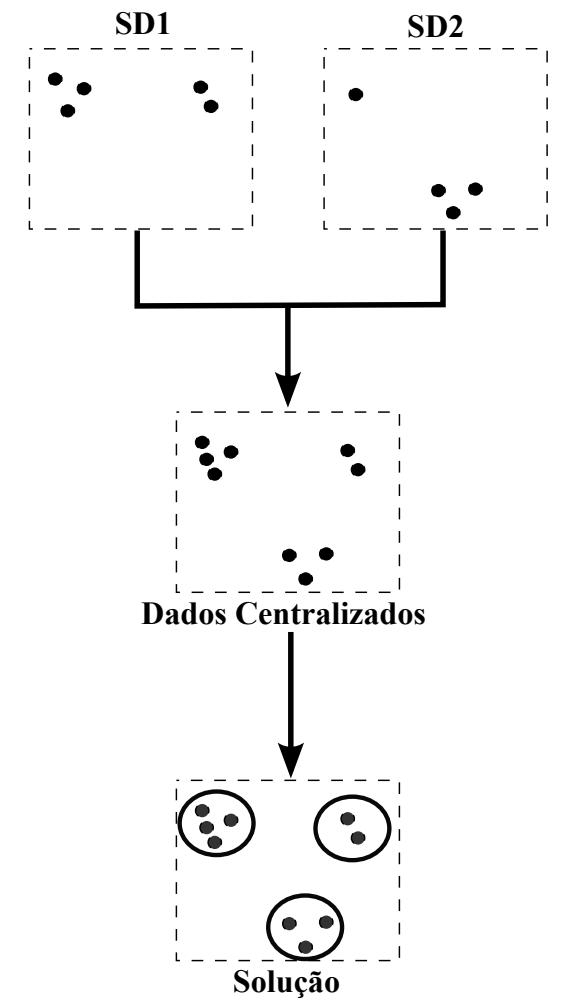

(a) Solução obtida com os dados centralizados.

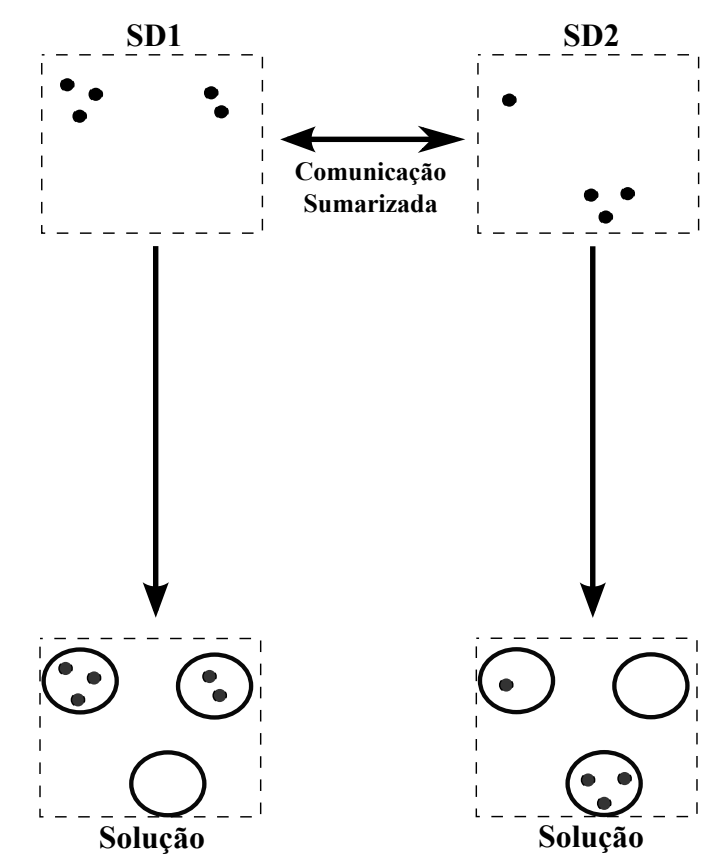

(b) Solução obtida sem a necessidade de centralização dos dados.

Figura 3.1: Agrupamento de dados com e sem a centralização dos dados.

os mesmos objetos, porém descritos em um espaço de atributos diferente. Nas Figuras 3.2 e 3.3 são ilustrados exemplos do caso horizontal e do caso vertical, respectivamente.

\begin{tabular}{|cccc|}
\hline \multicolumn{4}{c|}{ Tabela Global } \\
\hline ID & Nome & Cidade & Salário \\
\hline$\# 001-1$ & José & São Paulo & $\mathrm{R} \$ 1.787,00$ \\
$\# 001-2$ & Maria & São Paulo & $\mathrm{R} \$ 1.910,00$ \\
$\# 001-3$ & João & Rio de Janeiro & $\mathrm{R} \$ 2.346,00$ \\
$\# 001-4$ & Ana & Rio de Janeiro & $\mathrm{R} \$ 1.032,00$ \\
\hline$\# 002-1$ & Alberto & Belo Horizonte & $\mathrm{R} \$ 1.631,00$ \\
$\# 002-2$ & Carlos & Salvador & $\mathrm{R} \$ 1.281,00$ \\
$\# 002-3$ & Pedro & Salvador & $\mathrm{R} \$ 1.346,00$ \\
$\# 002-4$ & Joana & Belo Horizonte & $\mathrm{R} \$ 1.332,00$ \\
\hline
\end{tabular}

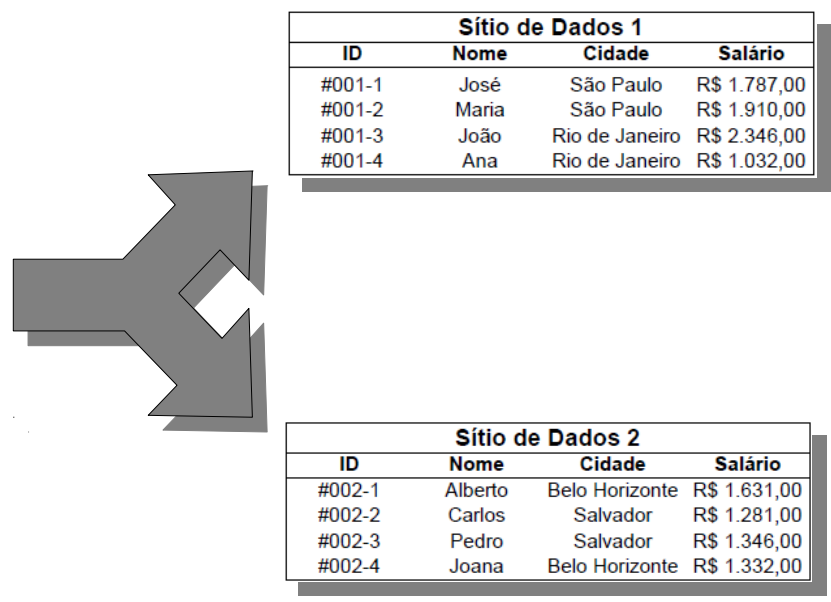

(a)

(b)

Figura 3.2: Dados (a) distribuídos horizontalmente (b).

Este capítulo dá mais enfoque às distribuições horizontais. Entretanto, na Seção 3.3 é apresentada uma abordagem para trabalhar com dados distribuídos verticalmente. 
(a)

\begin{tabular}{|c|ccc|cc|}
\hline \multicolumn{7}{|c|}{ Tabela Global } \\
\hline ID & Nome & Cidade & Salário & Peso & Altura \\
\hline$\# 001-1$ & José & São Paulo & $\mathrm{R} \$ 1.787,00$ & $70 \mathrm{~kg}$ & $1,75 \mathrm{~m}$ \\
$\# 001-2$ & Maria & São Paulo & $\mathrm{R} \$ 1.910,00$ & $60 \mathrm{~kg}$ & $1,60 \mathrm{~m}$ \\
$\# 001-3$ & João & Rio de Janeiro & $\mathrm{R} \$ 2.346,00$ & $81 \mathrm{~kg}$ & $1,70 \mathrm{~m}$ \\
$\# 001-4$ & Ana & Rio de Janeiro & $\mathrm{R} \$ 1.032,00$ & $54 \mathrm{~kg}$ & $1,59 \mathrm{~m}$ \\
$\# 002-1$ & Alberto & Belo Horizonte & $\mathrm{R} \$ 1.631,00$ & $102 \mathrm{~kg}$ & $1,81 \mathrm{~m}$ \\
$\# 002-2$ & Carlos & Salvador & $\mathrm{R} \$ 1.281,00$ & $89 \mathrm{~kg}$ & $1,69 \mathrm{~m}$ \\
$\# 002-3$ & Pedro & Salvador & $\mathrm{R} \$ 1.346,00$ & $73 \mathrm{~kg}$ & $1,72 \mathrm{~m}$ \\
$\# 002-4$ & Joana & Belo Horizonte & $\mathrm{R} \$ 1.332,00$ & $70 \mathrm{~kg}$ & $1,67 \mathrm{~m}$ \\
\hline
\end{tabular}

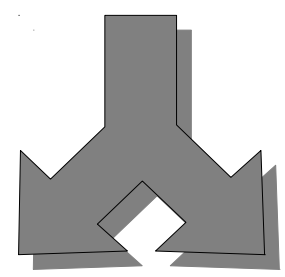

\begin{tabular}{|c|ccc|}
\hline \multicolumn{4}{|c|}{ Sítio de Dados 1 } \\
\hline ID & Nome & Cidade & Salário \\
\hline$\# 001-1$ & José & São Paulo & $\mathrm{R} \$ 1.787,00$ \\
$\# 001-2$ & Maria & São Paulo & $\mathrm{R} \$ 1.910,00$ \\
$\# 001-3$ & João & Rio de Janeiro & $\mathrm{R} \$ 2.346,00$ \\
$\# 001-4$ & Ana & Rio de Janeiro & $\mathrm{R} \$ 1.032,00$ \\
$\# 002-1$ & Alberto & Belo Horizonte & $\mathrm{R} \$ 1.631,00$ \\
$\# 002-2$ & Carlos & Salvador & $\mathrm{R} \$ 1.281,00$ \\
$\# 002-3$ & Pedro & Salvador & $\mathrm{R} \$ 1.346,00$ \\
$\# 002-4$ & Joana & Belo Horizonte & $\mathrm{R} \$ 1.332,00$ \\
\hline
\end{tabular}

\begin{tabular}{|c|cc|}
\hline \multicolumn{3}{|c|}{ Sítio de Dados 2 } \\
\hline ID & Peso & Altura \\
\hline$\# 001-1$ & $70 \mathrm{~kg}$ & $1,75 \mathrm{~m}$ \\
$\# 001-2$ & $60 \mathrm{~kg}$ & $1,60 \mathrm{~m}$ \\
$\# 001-3$ & $81 \mathrm{~kg}$ & $1,70 \mathrm{~m}$ \\
$\# 001-4$ & $54 \mathrm{~kg}$ & $1,59 \mathrm{~m}$ \\
$\# 002-1$ & $102 \mathrm{~kg}$ & $1,81 \mathrm{~m}$ \\
$\# 002-2$ & $89 \mathrm{~kg}$ & $1,69 \mathrm{~m}$ \\
$\# 002-3$ & $73 \mathrm{~kg}$ & $1,72 \mathrm{~m}$ \\
$\# 002-4$ & $70 \mathrm{~kg}$ & $1,67 \mathrm{~m}$ \\
\hline
\end{tabular}

(b)

Figura 3.3: Dados (a) distribuídos verticalmente (b).

\subsection{Generalização dos Algoritmos e Índices Estudados}

Vários algoritmos de agrupamento de dados foram propostos e investigados na literatura para encontrar estruturas em ambientes paralelos e distribuídos (Olson, 1995; Dhillon \& Modha, 2000; Tian et al., 2005; Du \& Lin, 2005; Garg et al., 2006; Hammouda \& Kamel, 2009; Datta et al., 2009). Mais especificamente, alguns foram desenvolvidos como generalizações de versões centralizadas de um algoritmo específico (Olson, 1995; Dhillon \& Modha, 2000; Forman \& Zhang, 2000; Garg et al., 2006), sendo capazes de produzir os mesmos resultados finais que seriam obtidos pelos respectivos algoritmos originais se estes pudessem ser aplicados aos dados de forma centralizada. Embora existam muitos algoritmos capazes de lidar com dados paralelos e distribuídos, poucos foram desenvolvidos para agrupamento fuzzy de dados como generalizações de versões centralizadas de determinado algoritmo (Kwok et al., 2002; Rahimi et al., 2004; Modenesi et al., 2007). Em outras palavras, poucos algoritmos de agrupamento fuzzy de dados foram generalizados para trabalhar com dados paralelos e distribuídos de forma a produzir os mesmos resultados finais que a versão centralizada de tal algoritmo obteria com os dados centralizados.

Nas próximas seções, estaremos particularmente interessados em variantes de algoritmos de agrupamento fuzzy centralizados concebidos para manipular dados distribuídos 
(naturalmente ou artificialmente). Formalmente, seja $\mathbf{X}[i i]=\left\{\mathbf{x}[i i]_{1}, \ldots, \mathbf{x}[i i]_{N_{i i}}\right\}$ uma base de dados, no $i i$-ésimo sítio de dados, composta de $N_{i i}$ objetos, $\mathbf{x}[i i]_{j}\left(j=1, \ldots, N_{i i}\right)$, cada qual descrito em um espaço de atributos $n$-dimensional. Então, considere um cenário no qual exista uma coleção distribuída de tais bases, $\mathbf{X}[1], \ldots, \mathbf{X}[P]$, todas descritas no mesmo espaço de atributos $n$-dimensional (distribuição horizontal). O objetivo de um algoritmo de agrupamento fuzzy distribuído, como uma generalização de um algoritmo centralizado, consiste em encontrar uma solução que, simultaneamente, represente uma estrutura comum a todos os sítios de dados. Em outras palavras, o algoritmo procura por uma matriz de partição $\mathbf{U}$ com $k$ grupos que representam as pertinências de cada um dos $N_{1}+N_{2}+\cdots+N_{P}$ objetos aos $k$ grupos. O resultado final (U) é presumido ser exatamente o mesmo resultado que seria obtido pelo algoritmo original (centralizado) se tal algoritmo fosse executado em uma única base de dados contendo todos os objetos de todos os sítios de dados $(\mathbf{X}=\mathbf{X}[1] \cup \mathbf{X}[2] \cup \cdots \cup \mathbf{X}[P])$.

\subsubsection{DFCM: Distributed Fuzzy c-Means}

O algoritmo denominado DFCM (Distributed Fuzzy c-Means - em inglês) foi originalmente proposto no contexto paralelo (Kwok et al., 2002; Rahimi et al., 2004; Modenesi et al., 2007). Este algoritmo consiste na generalização do algoritmo FCM (Seção 2.2.1) para lidar com dados paralelos ou distribuídos. Note que o Algoritmo 1 (FCM na Seção 2.2.1) consiste basicamente de três passos: (i) o cálculo dos protótipos; (ii) o cálculo das distâncias; e (iii) a atualização da matriz de partição. Os protótipos são calculados de acordo com (2.10), que necessita de todos os objetos. Reescrevendo esta equação para utilizar objetos distribuídos em $P$ sítios de dados, obtém-se $(1 \leq i \leq k)$ :

$$
\mathbf{v}_{i}=\frac{\sum_{i i=1}^{P} \sum_{j=1}^{N_{i i}}\left(u[i i]_{i j}\right)^{m} \mathbf{x}[i i]_{j}}{\sum_{i i=1}^{P} \sum_{j=1}^{N_{i i}}\left(u[i i]_{i j}\right)^{m}}
$$

em que $N_{i i}$ é o número de objetos no $i i$-ésimo sítio de dados, $\mathbf{x}[i i]_{j}$ é o $j$-ésimo objeto do $i i$ ésimo sítio de dados, $u[i i]_{i j}$ é a pertinência do $j$-ésimo objeto do $i i$-ésimo sítio de dados ao $i$ ésimo grupo e $\mathbf{v}_{i}$ é o protótipo do $i$-ésimo grupo, denominado como protótipo global porque é obtido utilizando todos os objetos de todos os sítios de dados e consiste exatamente no mesmo protótipo que seria obtido por (2.10) se os dados estivessem centralizados.

O cálculo dos protótipos globais em (3.1) necessita de todos os objetos de todos os sítios de dados, o que pode ser impraticável em cenários paralelos e distribuídos. Entretanto, cada sítio de dados pode calcular os protótipos parciais (locais) considerando somente os seus objetos como $(1 \leq i i \leq P, 1 \leq i \leq k)$ :

$$
\mathbf{v}[i i]_{i}=\frac{\sum_{j=1}^{N_{i i}}\left(u[i i]_{i j}\right)^{m} \mathbf{x}[i i]_{j}}{\sum_{j=1}^{N_{i i}}\left(u[i i]_{i j}\right)^{m}}
$$

Aqui, $\mathbf{v}[i i]_{i}$ é denominado o $i$-ésimo protótipo local do $i i$-ésimo sítio de dados porque é 
obtido estritamente dos objetos do $i i$-ésimo sítio de dados. Denotaremos por "somas das pertinências" o denominador de (3.2), i.e., $(1 \leq i \leq k)$ :

$$
\mu[i i]_{i}=\sum_{j=1}^{N_{i i}}\left(u[i i]_{i j}\right)^{m} .
$$

Agora, se todos os sítios de dados enviarem seus protótipos locais e as somas das pertinências para um sítio de dados central ${ }^{2}$, então é possível calcular os protótipos globais como $(1 \leq i \leq k)$ :

$$
\mathbf{v}_{i}=\frac{\sum_{i i=1}^{P} \mathbf{v}[i i]_{i} \cdot \mu[i i]_{i}}{\sum_{i i=1}^{P} \mu[i i]_{i}}
$$

Finalmente, o sítio de dados central envia os protótipos globais a todos os demais sítios de dados. Dessa maneira, todos os sítios de dados têm acesso aos mesmos protótipos globais.

O próximo passo do algoritmo FCM consiste no cálculo das distâncias entre objetos e protótipos. Note que, com acesso aos protótipos globais, cada sítio de dados pode calcular as distâncias entre seus objetos e cada protótipo como $(1 \leq i i \leq P, 1 \leq i \leq k, 1 \leq j \leq N)$ :

$$
D[i i]_{i j}=\left\|\mathbf{x}[i i]_{j}-\mathbf{v}_{i}\right\|_{\mathbf{A}}^{2}=\left(\mathbf{x}[i i]_{j}-\mathbf{v}_{i}\right)^{T} \mathbf{A}\left(\mathbf{x}[i i]_{j}-\mathbf{v}_{i}\right) .
$$

Este cálculo não requer nenhuma comunicação extra entre os sítios de dados. Uma vez que é realizado, cada sítio de dados possui as distâncias entre seus objetos e os protótipos globais.

O passo final do algoritmo FCM consiste em atualizar a matriz de partição. Note que o cálculo das pertinências de um dado objeto $\mathbf{x}_{j}$ em (2.8) depende apenas das distâncias entre este objeto e os protótipos dos grupos. Em outras palavras, as distâncias entre os protótipos e os outros objetos (ainda que no mesmo sítio de dados) não são necessárias para o cálculo de pertinência de um objeto particular $\mathbf{x}[i i]_{j}$. Assim, a matriz de partição pode ser atualizada como $(1 \leq i i \leq P, 1 \leq i \leq k, 1 \leq j \leq N)$ :

$$
u[i i]_{i j}=\left(\sum_{c=1}^{k}\left(\frac{D[i i]_{i j}}{D[i i]_{c j}}\right)^{1 /(m-1)}\right)^{-1},
$$

sem a necessidade de qualquer comunicação entre os sítios de dados.

Em resumo, somente o cálculo dos protótipos requer comunicações entre os sítios de dados. Os passos referentes ao cálculo das distâncias e à atualização da matriz de partição não requerem qualquer comunicação extra. Com base nessas considerações, a versão paralela/distribuída do algoritmo FCM é apresentada no Algoritmo 25 (cada sítio de dados, incluindo o sítio central, deve executar o mesmo procedimento).

\footnotetext{
${ }^{2}$ Um sítio de dados escolhido para ser o sítio central.
} 


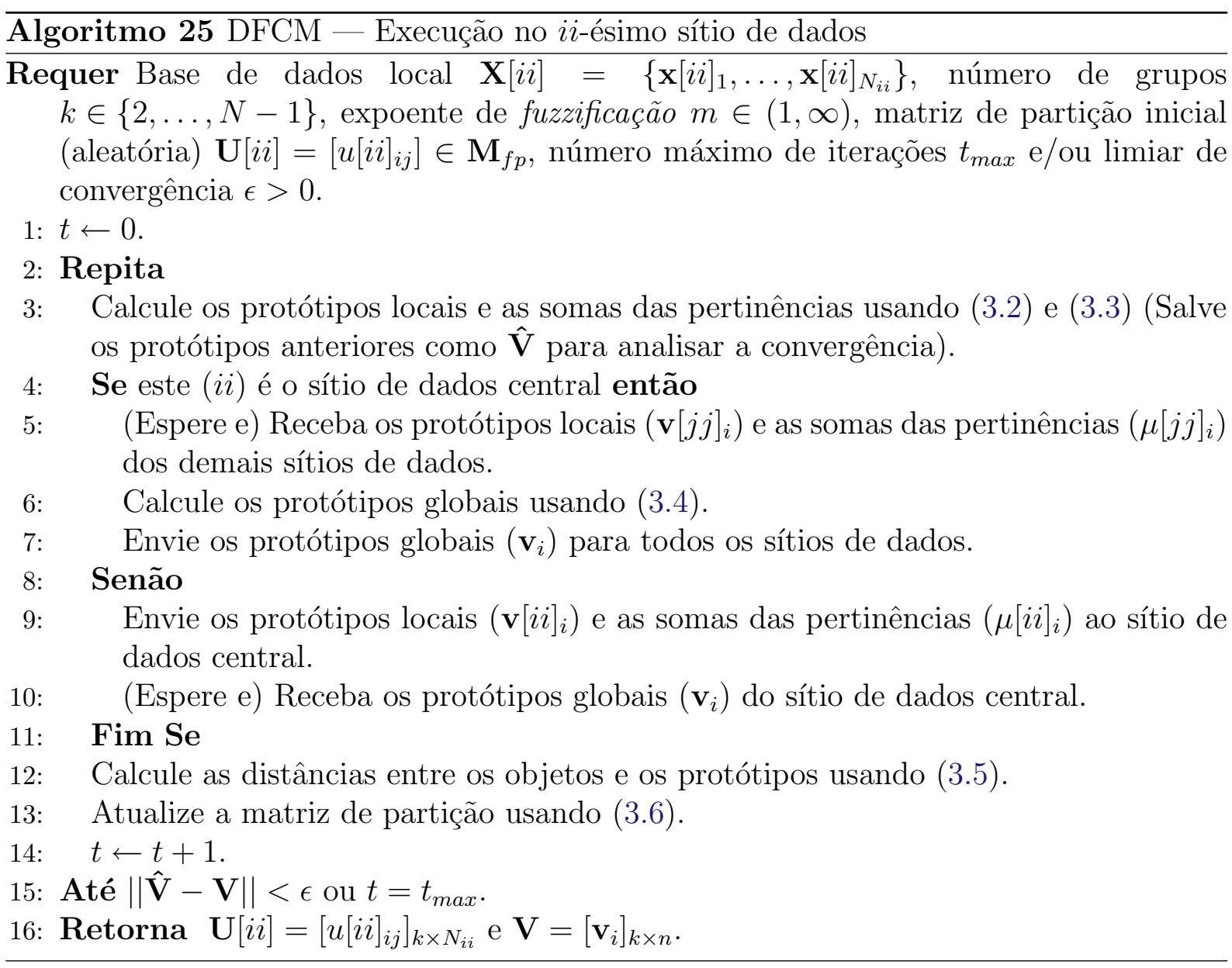

Alguns pontos importantes a serem destacados são:

1. O algoritmo DFCM comunica apenas os protótipos e as somas das pertinências. Nenhuma informação sobre os objetos originais é comunicada entre os sítios de dados.

2. Quando os mesmos parâmetros iniciais são usados, o algoritmo DFCM resulta na mesma partição final que o algoritmo FCM resultaria se todos os objetos estivessem centralizados.

3. A convergência $\|\hat{\mathbf{V}}-\mathbf{V}\|<\epsilon$ analisa os protótipos globais. Se desejado, é possível analisar a convergência usando a matriz de partição $\|\hat{\mathbf{U}}[i i]-\mathbf{U}[i i]\|<\epsilon$. Note, entretanto, que desta forma é necessário uma comunicação adicional de um bit para checar se $\|\hat{\mathbf{U}}[i i]-\mathbf{U}[i i]\|<\epsilon \forall i i$.

4. Os Passos 3 a 11 do Algoritmo 25 estão relacionados ao Passo 3 do Algoritmo 1, o Passo 12 do Algoritmo 25 está relacionado ao Passo 4 do Algoritmo 1 e o Passo 13 do Algoritmo 25 está relacionado ao Passo 5 do Algoritmo 1.

\subsubsection{Framework para Dados Distribuídos}

Vários dos algoritmos apresentados no Capítulo 2 podem ser generalizados para manipular dados paralelos e distribuídos de maneira similar ao algoritmo DFCM, i.e., os passos 
do algoritmo original (centralizado) são executados e, quando um passo em particular requer comunicação entre os sítios de dados, os valores locais são calculados e enviados a um sítio de dados central, que, por sua vez, calcula os valores globais e envia de volta a todos os outros sítios de dados.

Mais especificamente, o framework proposto neste documento executa cada passo do algoritmo original (centralizado) e, se um passo de comunicação é necessário, então ele procede da seguinte forma:

1. Cada sítio de dados calcula seus respectivos valores locais $V[i i]$.

2. Cada sítio de dados envia seus valores locais para um sítio de dados central.

3. O sítio de dados central calcula os valores globais como:

$$
V=\frac{\left[\sum_{i i=1}^{P} \alpha[i i] \cdot(V[i i]-\Delta) \cdot \phi[i i]\right]+\Delta}{\sum_{i i=1}^{P} \alpha[i i] \cdot \psi[i i]}
$$

4. O sítio de dados central envia os valores globais de volta a todos os outros sítios de dados.

Em (3.7), $V$ é o valor global a ser calculado pelo sítio de dados central e enviado aos outros sítios, $P$ é o número de sítios de dados, $V[i i]$ é o valor local calculado pelo $i i$-ésimo sítio de dados, $\Delta$ é um valor que pode ser calculado pelo sítio central sem a necessidade de comunicação extra, $\phi[i i]$ e $\psi[i i]$ são valores adicionais que devem ser enviados pelo $i i$-ésimo sítio de dados ao sítio central e $\alpha[i i]$ é um parâmetro opcional que define a importância associada a um determinado $i i$-ésimo sítio de dados. Por questão de simplicidade, nos desenvolvimentos a seguir serão adotados $\alpha[i i]=1 \forall i i$ (todos os sítios apresentam a mesma importância relativa). Dessa forma (3.7) pode ser reescrita como:

$$
V=\frac{\left[\sum_{i i=1}^{P}(V[i i]-\Delta) \cdot \phi[i i]\right]+\Delta}{\sum_{i i=1}^{P} \psi[i i]} .
$$

Em resumo, quando um passo de comunicação é necessário, o respectivo passo do algoritmo original (centralizado) deve ser substituído pelos passos ilustrados no Algoritmo 26. Por exemplo, o algoritmo DFCM pode ser obtido utilizando este método. Conforme explicado anteriormente, o algoritmo FCM consiste de três passos. Dois deles não requerem comunicação, a saber, o cálculo das distâncias entre os objetos e os protótipos e a atualização da matriz de partição. O cálculo dos protótipos necessita comunicação entre os sítios de dados e pode ser realizada usando o Algoritmo 26 definindo:

- $V=\mathbf{v}_{i}$ (protótipos globais).

- $V[i i]=\mathbf{v}[i i]_{i}$ (protótipos locais).

- $\phi[i i]=\psi[i i]=\sum_{j=1}^{N_{i i}}\left(u[i i]_{i j}\right)^{m}$ (somas das pertinências $\left.\mu[i i]_{i}\right)$. 
- $\Delta=0$.

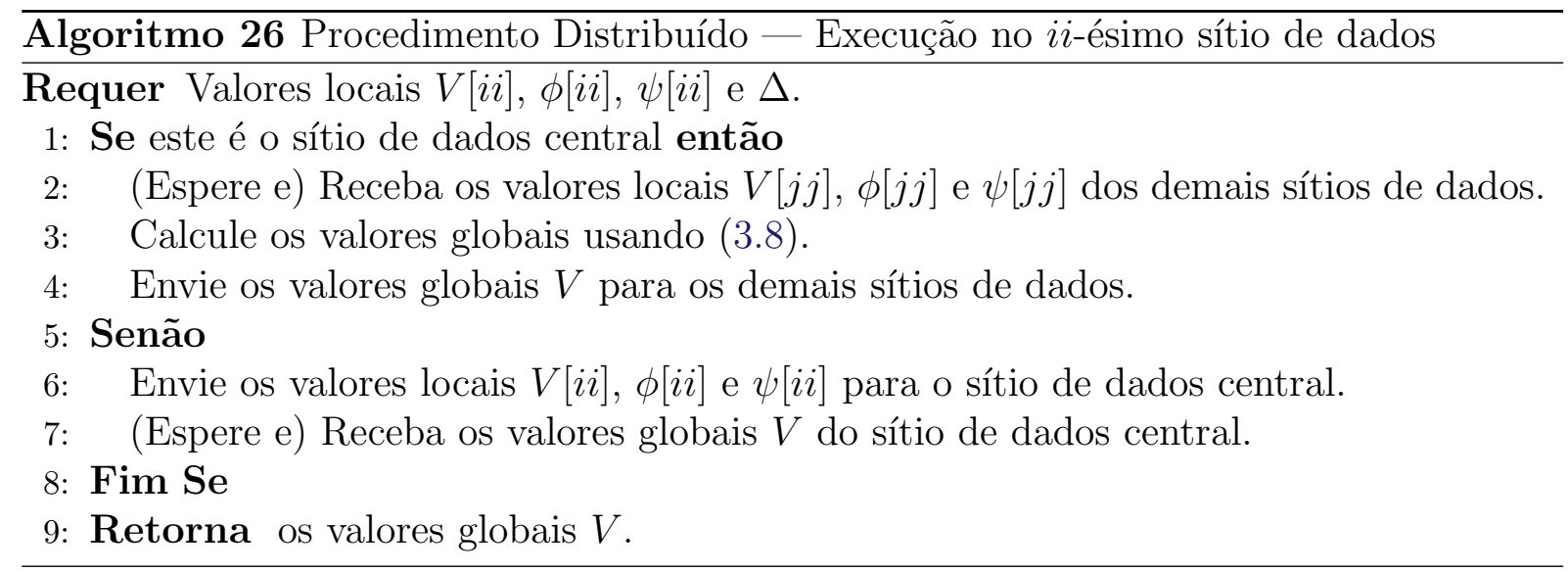

\subsubsection{Outros Algoritmos de Agrupamento Fuzzy Distribuídos}

A mesma ideia discutida anteriormente com respeito ao algoritmo DFCM também pode ser aplicada a outros algoritmos de agrupamento fuzzy revisados no Capítulo 2. Por exemplo, a versão distribuída do algoritmo GK (Seção 2.2.2.1), denominada DGK, necessita calcular os protótipos globais (usando (3.4)) e as matrizes de covariância globais $(1 \leq i \leq k)$ :

$$
\mathbf{F}_{i}=\frac{\sum_{i i=1}^{P} \sum_{j=1}^{N_{i i}}\left(u[i i]_{i j}\right)^{m}\left(\mathbf{x}[i i]_{j}-\mathbf{v}_{i}\right)\left(\mathbf{x}[i i]_{j}-\mathbf{v}_{i}\right)^{T}}{\sum_{i i=1}^{P} \sum_{j=1}^{N_{i i}}\left(u[i i]_{i j}\right)^{m}} .
$$

A mesma estratégia usada para calcular os protótipos globais pode ser usada para calcular as matrizes de covariância globais. De fato, note que definindo $V=\mathbf{F}_{i}, V[i i]=$ $\mathbf{F}[i i]_{i}, \Delta=0$, e $\psi[i i]=\phi[i i]=\sum_{j=1}^{N_{i i}}\left(u[i i]_{i j}\right)^{m}$, as matrizes de covariância globais podem ser calculadas usando (3.8) reescrevendo-as por $(1 \leq i \leq k)$ :

$$
\mathbf{F}_{i}=\frac{\sum_{i i=1}^{P} \mathbf{F}[i i]_{i} \cdot \phi[i i]}{\sum_{i i=1}^{P} \psi[i i]}
$$

em que $(1 \leq i i \leq P, 1 \leq i \leq k)$

$$
\mathbf{F}[i i]_{i}=\frac{\sum_{j=1}^{N_{i i}}\left(u[i i]_{i j}\right)^{m}\left(\mathbf{x}[i i]_{j}-\mathbf{v}_{i}\right)\left(\mathbf{x}[i i]_{j}-\mathbf{v}_{i}\right)^{T}}{\sum_{j=1}^{N_{i i}}\left(u[i i]_{i j}\right)^{m}}
$$

são as matrizes de covariância locais do ii-ésimo sítio de dados (matrizes de covariância calculadas utilizando somente os objetos do $i i$-ésimo sítio de dados). 
Uma vez que os protótipos e as matrizes de covariância globais foram calculados, as distâncias defindas por $(1 \leq i i \leq P, 1 \leq i \leq k, 1 \leq j \leq N)$

$$
D[i i]_{i j}=\left\|\mathbf{x}[i i]_{j}-\mathbf{v}_{i}\right\|_{\mathbf{A}_{i}}^{2}=\left(\mathbf{x}[i i]_{j}-\mathbf{v}_{i}\right)^{T} \mathbf{A}_{i}\left(\mathbf{x}[i i]_{j}-\mathbf{v}_{i}\right)
$$

(com $\mathbf{A}_{i}$ definido em (2.12)) e os valores de pertinências definidos por (3.6) podem ser obtidos. O procedimento completo do algoritmo DGK é ilustrado no Algoritmo 27.

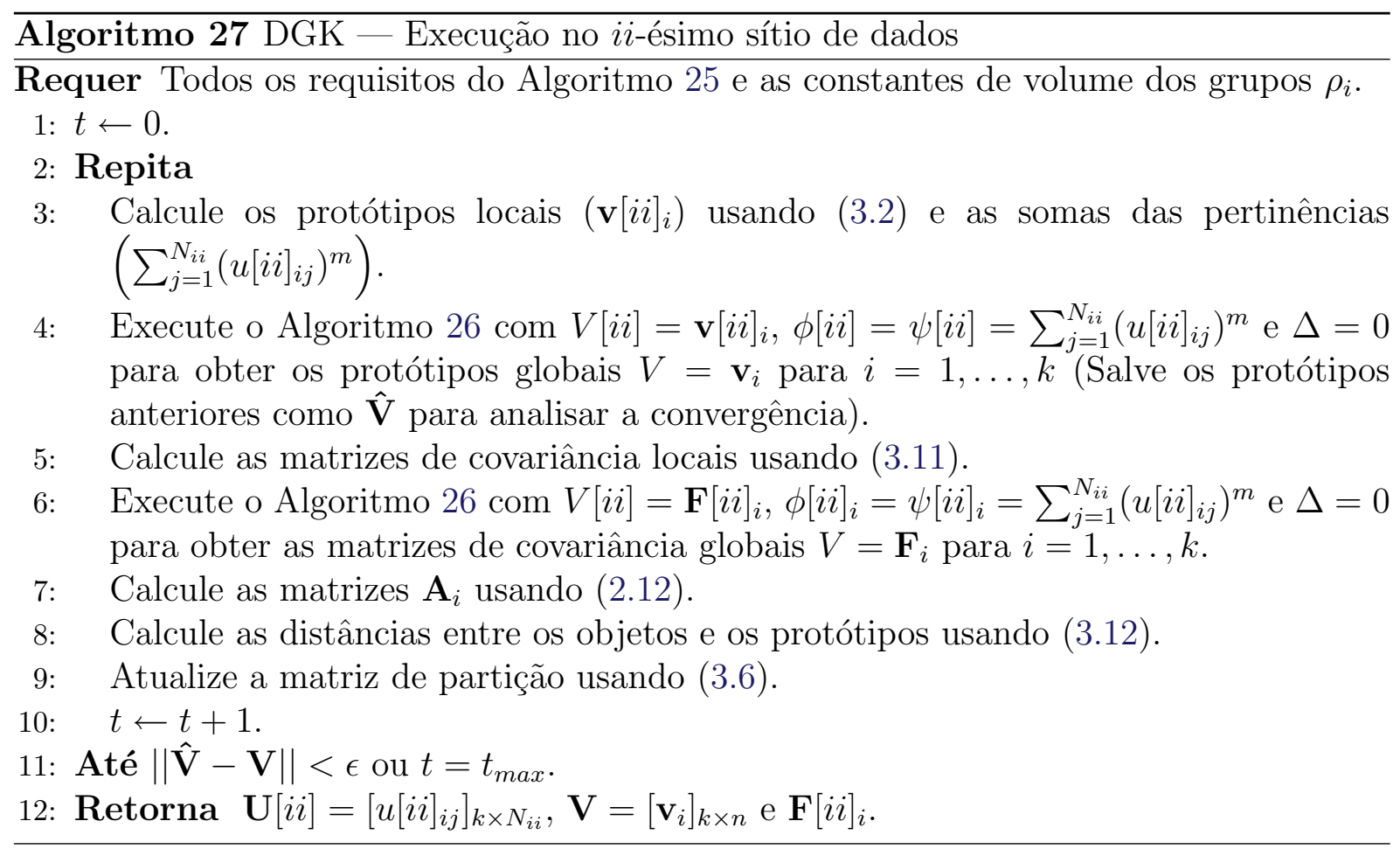

Além dos algoritmos FCM (Seção 2.2.1) e GK (Seção 2.2.2.1), os algoritmos GG (Seção 2.2.2.2), FCV (Seção 2.2.3.1), FCE (Seção 2.2.3.2), EFCM (Seção 2.2.3.3), PCM (Seção 2.2.5.1), PGK (Seção 2.2.5.2), FPCM (Seção 2.2.5.3), PFCM (Seção 2.2.5.4), WDSFCM (Seção 2.2.7.1), PDSFCM (Seção 2.2.7.2), OCSFCM (Seção 2.2.7.3), NPSFCM (Seção 2.2.7.4), brFCM (Seção 2.2.8.1), mrFCM (Seção 2.2.8.2) e FSCM (Seção 2.2.9.1), descritos no Capítulo 2, podem ser diretamente generalizados para trabalhar com dados distribuídos seguindo a mesma ideia. Entretanto, é importante notar que a versão distribuída do algoritmo FPCM (DFPCM) requer comunicações adicionais em cada iteração para atualizar as tipicidades. De fato, note que o algoritmo FPCM requer o cálculo de $\varphi_{i}$ em (2.39), o que pode ser obtido por $(1 \leq i \leq k)$ :

$$
\varphi_{i}=\sum_{i i=1}^{P} \varphi[i i]_{i},
$$

i.e., usando (3.8) definindo $V=\varphi_{i}, V[i i]=\varphi[i i]_{i}, \Delta=0, \phi[i i]=1$ e $\psi[i i]=1 / P$, em que

$$
\varphi[i i]_{i}=\sum_{l=1}^{N_{i i}}\left(\frac{1}{D[i i]_{i l}}\right)^{1 /(\gamma-1)}
$$


são calculados localmente em cada sítio de dados e enviados ao sítio central.

Na Seção 3.1.7 estão resumidas as configurações dos termos da Equação (3.8) para a utilização desses algoritmos em cenários distribuídos.

\subsubsection{Análise de Complexidade - Comunicação}

Dado que a complexidade de tempo e espaço dos algoritmos distribuídos seguem as análises de complexidades relatadas no Capítulo $2^{3}$, aqui será apresentada apenas a análise de complexidade de comunicação de cada algoritmo.

Seguindo o procedimento distribuído em (3.8), é necessário comunicar três variáveis: $V[i i], \phi[i i]$ e $\psi[i i]$. O tamanho da primeira depende do que se está comunicando. As outras são valores reais e requerem a comunicação de um único valor cada. Dessa maneira, a complexidade de comunicação depende da definição de $V[i i]$.

Para calcular os protótipos globais, é necessário comunicar $O(n+1+1)$ valores para cada grupo. Assim, a complexidade de comunicação total para o cálculo dos protótipos globais é $O(k(n+1+1)) \rightarrow O(k n)$. Para calcular as matrizes de covariância globais é necessário comunicar $O\left(n^{2}+1+1\right)$ valores para cada grupo. Dessa forma, a complexidade de comunicação total para o cálculo das matrizes de covariância globais é $O\left(k\left(n^{2}+1+\right.\right.$ $1)) \rightarrow O\left(k n^{2}\right)$. Para calcular os valores globais $\varphi_{i}$ em (2.39) é necessário comunicar $O(k)$ valores, o que pode ficar mais claro a partir de (3.13) e (3.14). Analogamente, para calcular as probabilidades a priori em (2.15) é necessário comunicar $O(k)$ valores. Para o cálculo do (tamanho do maior intervalo do $p$-ésimo atributo) em (2.70) para todos os atributos é necessário apenas uma comunicação de $O(n)$ valores antes da execução do algoritmo. Dessas análises de complexidades é possível obter a complexidade de comunicação de cada algoritmo distribuído. As complexidades estão sumarizadas nas Tabelas 3.1 e 3.2. Também estão ilustrados os elementos a serem comunicados pelos algoritmos ${ }^{4}$.

\subsubsection{Validação Distribuída de Agrupamento}

Os índices revisados na Seção 2.5 assumem que os dados estão centralizados. Note, contudo, que tais índices dependem essencialmente: (i) das pertinências dos objetos aos grupos; (ii) das distâncias entre protótipos; e (iii) das distâncias entre os objetos e os protótipos. Conforme discutido na Seção 3.1.1, (i) e (iii) podem ser calculados localmente em cada sítio de dados e então comunicados para um sítio central, enquanto (ii) pode ser facilmente obtido pois cada sítio de dados possui acesso aos protótipos globais. Novamente, quando necessário, (3.8) pode ser usada para calcular os valores globais. Assim, as versões distribuídas de cada índice de validade pode ser calculada. Baseado nessa

\footnotetext{
${ }^{3}$ Note que agora o número de objetos é dado por $N_{i i}$.

${ }^{4}$ Não estão ilustrados os valores de $\phi[i i]$ e $\psi[i i]$ na Tabela 3.1 pois eles são valores escalares e, como tal, não modificam a complexidade de comunicação. Para maiores detalhes sobre os cálculos de tais valores, veja a Seção 3.1.7.
} 


\begin{tabular}{lcc} 
Algoritmo & $\begin{array}{c}\text { Elementos principais a } \\
\text { serem comunicados }\end{array}$ & Complexidade de Comunicação \\
DFCM & $\mathbf{v}_{i}$ & $O(P t k n)$ \\
DGK & $\mathbf{v}_{i}$ e $\mathbf{F}_{i}$ & $O\left(P t\left(k n+k n^{2}\right)\right) \rightarrow O\left(P t k n^{2}\right)$ \\
DGG & $\mathbf{v}_{i}, P_{i}$ e $\mathbf{F}_{i}$ & $O\left(P t\left(k n+k+k n^{2}\right)\right) \rightarrow O\left(P t k n^{2}\right)$ \\
DFCV & $\mathbf{v}_{i}$ e $\mathbf{F}_{i}$ & $O\left(P t\left(k n+k n^{2}\right)\right) \rightarrow O\left(P t k n^{2}\right)$ \\
DFCE & $\mathbf{v}_{i}$ e $\mathbf{F}_{i}$ & $O\left(P t\left(k n+k n^{2}\right)\right) \rightarrow O\left(P t k n^{2}\right)$ \\
DEFCM & $\mathbf{v}_{i}$ e $\mathbf{F}_{i}$ & $O\left(P t\left(k n+k n^{2}\right)\right) \rightarrow O\left(P t k n^{2}\right)$ \\
DPCM & $\mathbf{v}_{i}$ & $O(P t k n)$ \\
DPGK & $\mathbf{v}_{i}$ e $\mathbf{F}_{i}$ & $O\left(P t\left(k n+k n^{2}\right)\right) \rightarrow O\left(P t k n^{2}\right)$ \\
DFPCM & $\mathbf{v}_{i}$ e $D_{i l}$ & $O(P t k n)$ \\
\hline
\end{tabular}

Tabela 3.1: Complexidade de comunicação para os algoritmos de agrupamento fuzzy distribuídos: $P$ é o número de sítios de dados, $t$ é o número de iterações, $k$ é o número de grupos e $n$ é o número de atributos.

ideia, nesta seção os índices revisados na Seção 2.5 são generalizados para lidar com dados distribuídos.

\subsubsection{DPC: Distributed Partition Coefficient}

O índice PC em (2.74) pode ser reescrito como:

$$
V_{D P C}=\frac{1}{N} \sum_{i i=1}^{P} \sum_{i=1}^{k} \sum_{j=1}^{N_{i i}}\left(u[i i]_{i j}\right)^{2},
$$

em que $N=N_{1}+N_{2}+\cdots+N_{P}$, $P$ é o número de sítio de dados, $k$ é o número de grupos, $N_{i i}$ é o número de objetos no $i i$-ésimo sítio de dados e $u[i i]_{i j}$ é a pertinência do $j$-ésimo objeto ao $i$-ésimo grupo no $i i$-ésimo sítio de dados. Entretanto, este índice pode ser calculado com os dados distribuídos usando (3.8). Seja $V=V_{D P C}$,

$$
V[i i]=V_{P C}[i i]=\frac{1}{N_{i i}} \sum_{i=1}^{k} \sum_{j=1}^{N_{i i}}\left(u[i i]_{i j}\right)^{2}
$$




\begin{tabular}{lcc} 
Algoritmo & $\begin{array}{c}\text { Elementos principais a } \\
\text { serem comunicados }\end{array}$ & Complexidade de Comunicação \\
DPFCM & $\mathbf{v}_{i}$ & $O($ Ptkn $)$ \\
DWDSFCM & $\mathbf{v}_{i}$ & O(Ptkn $)$ \\
DPDSFCM & $\mathbf{v}_{i p}$ & $O(P t k n)$ \\
DOCSFCM & $\mathbf{v}_{i}$ & $O(P t k n)$ \\
DNPSFCM & $\mathbf{v}_{i}$ & $O(P t k n)$ \\
DbrFCM & $\mathbf{v}_{i}$ & $O(P t k n)$ \\
DmrFCM & $\mathbf{v}_{i}$ & $O(P r t k n)$ \\
DFSCM & $e_{i, p, l}$ & $O\left(P t k n_{p} n\right)$ \\
\hline
\end{tabular}

Tabela 3.2: Complexidade de comunicação para os algoritmos de agrupamento fuzzy distribuídos: $P$ é o número de sítios de dados, $t$ é o número de iterações, $k$ é o número de grupos, $n$ é o número de atributos, $r$, no algoritmo DmrFCM, é o número de amostras e $n_{p}$, no algoritmo DFSCM, é a quantidade de valores diferentes do $p$-ésimo atributo.

o índice calculado localmente no $i i$-ésimo sítio de dados, $\Delta=0$ e $\phi[i i]=\psi[i i]=N_{i i}$, então a versão distribuída do índice PC pode ser calculada como:

$$
V_{D P C}=\frac{\sum_{i i=1}^{P} V_{P C}[i i] \cdot N_{i i}}{\sum_{i i=1}^{P} N_{i i}}
$$

\subsubsection{DPE: Distributed Partition Entropy}

O índice PE em (2.75) pode ser reescrito como:

$$
V_{D P E}=-\frac{1}{N} \sum_{i i=1}^{P} \sum_{i=1}^{k} \sum_{j=1}^{N_{i i}} u[i i]_{i j} \cdot \log _{a}\left(u_{i j}\right),
$$

em que $N=N_{1}+N_{2}+\cdots+N_{P}$. Contudo, este índice pode ser calculado com dados distribuídos usando (3.8). Seja $V=V_{D P E}$,

$$
V[i i]=V_{P E}[i i]=-\frac{1}{N_{i i}} \sum_{i=1}^{k} \sum_{j=1}^{N_{i i}} u[i i]_{i j} \cdot \log _{a}\left(u_{i j}\right)
$$


o índice calculado localmente usando (2.75) no $i$-ésimo sítio de dados, $\Delta=0$ e $\phi[i i]=$ $\psi[i i]=N_{i i}$, então a versão distribuída do índice PE pode ser calculada como:

$$
V_{D P E}=\frac{\sum_{i i=1}^{P} V_{P E}[i i] \cdot N_{i i}}{\sum_{i i=1}^{P} N_{i i}}
$$

\subsubsection{DMPC: Distributed Modified Partition Coefficient}

A versão distribuída do índice MPC, DMPC, pode ser facilmente calculada usando o índice DPC (Seção 3.1.5.1):

$$
V_{D M P C}=1-\frac{k}{k-1}\left(1-V_{D P C}\right)
$$

\subsubsection{DXB: Distributed Xie-Beni}

O índice XB em (2.77) pode ser reescrito como:

$$
V_{D X B}=\frac{\sum_{i i=1}^{P} \sum_{i=1}^{k} \sum_{j=1}^{N_{i i}}\left(u[i i]_{i j}\right)^{m}\left\|\mathbf{x}[i i]_{j}-\mathbf{v}_{i}\right\|^{2}}{\sum_{i i=1}^{P} N_{i i} \min _{l \neq s}|| \mathbf{v}_{l}-\mathbf{v}_{s} \|^{2}}
$$

Este índice pode ser calculado com dados distribuídos usando (3.8), definindo $V=V_{D X B}$, $\Delta=0, \phi[i i]=\psi[i i]=N_{i i}$ e $V[i i]=V_{X B}[i i]$, em que $V_{X B}[i i]$ é o índice calculado localmente usando (2.77) no $i$-ésimo sítio de dados, i.e:

$$
V_{X B}[i i]=\frac{\sum_{i=1}^{k} \sum_{j=1}^{N_{i i}}\left(u[i i]_{i j}\right)^{m}\left\|\mathbf{x}[i i]_{j}-\mathbf{v}_{i}\right\|^{2}}{N_{i i} \min _{l \neq s}\left\|\mathbf{v}_{l}-\mathbf{v}_{s}\right\|^{2}} .
$$

Dessa forma, a versão distribuída do índice XB pode ser calculada como (Vendramin et al., 2011):

$$
V_{D X B}=\frac{\sum_{i i=1}^{P} V_{X B}[i i] \cdot N_{i i}}{\sum_{i i=1}^{P} N_{i i}}
$$

\subsubsection{DFSS: Distributed Fuzzy Simplified Silhouette}

O índice FSS em (2.78) pode ser reescrito como:

$$
V_{D F S S}=\frac{\sum_{i i=1}^{P} \sum_{j=1}^{N_{i i}}\left(u[i i]_{p j}-u[i i]_{q j}\right)^{\alpha} s[i i]_{j}}{\sum_{i i=1}^{P} \sum_{j=1}^{N_{i i}}\left(u[i i]_{p j}-u[i i]_{q j}\right)^{\alpha}},
$$

em que $s[i i]_{j}$ é a silhueta do $j$-ésimo objeto do $i i$-ésimo sítio de dados e, dado que envolve apenas distâncias entre o objeto $\mathbf{x}[i i]_{j}$ e os protótipos, pode ser calculada sem comunicação extra. Agora, seja $V=V_{D F S S}, \Delta=0, \phi[i i]=\psi[i i]=\sum_{j=1}^{N_{i i}}\left(u[i i]_{p j}-u[i i]_{q j}\right)^{\alpha}$ e $V[i i]=$ 
$V_{F S S}[i i]$, em que $V_{F S S}[i i]$ é o índice calculado localmente usando (2.78) no ii-ésimo sítio de dados, i.e.:

$$
V_{F S S}[i i]=\frac{\sum_{j=1}^{N_{i i}}\left(u[i i]_{p j}-u[i i]_{q j}\right)^{\alpha} s[i i]_{j}}{\sum_{j=1}^{N_{i i}}\left(u[i i]_{p j}-u[i i]_{q j}\right)^{\alpha}}
$$

então, a versão distribuída do índice FSS pode ser calculada como:

$$
V_{D F S S}=\frac{\sum_{i i=1}^{P} V_{F S S}[i i] \cdot \phi[i i]}{\sum_{i i=1}^{P} \psi[i i]}=\frac{\sum_{i i=1}^{P} V_{F S S}[i i] \cdot \sum_{j=1}^{N_{i i}}\left(u[i i]_{p j}-u[i i]_{q j}\right)^{\alpha}}{\sum_{i i=1}^{P} \sum_{j=1}^{N_{i i}}\left(u[i i]_{p j}-u[i i]_{q j}\right)^{\alpha}} .
$$

\subsubsection{DK: Distributed Kwon}

O índice K em (2.80) pode ser reescrito como:

$$
V_{D K}=\frac{\sum_{i i=1}^{P} \sum_{i=1}^{k} \sum_{j=1}^{N_{i i}}\left(u[i i]_{i j}\right)^{m}\left\|\mathbf{x}[i i]_{j}-\mathbf{v}_{i}\right\|^{2}}{\min _{l \neq s}|| \mathbf{v}_{l}-\mathbf{v}_{s} \|^{2}}+\frac{\frac{1}{k} \sum_{i=1}^{k}\left\|\mathbf{v}_{i}-\overline{\mathbf{x}}\right\|^{2}}{\min _{l \neq s}|| \mathbf{v}_{l}-\mathbf{v}_{s} \|^{2}},
$$

em que $\overline{\mathbf{x}}=\frac{\sum_{i i=1}^{P} \sum_{j=1}^{N_{i i} \mathbf{x}}[i i]_{j}}{\sum_{i i=1}^{P} N_{i i}}$ é a média geral, que pode ser calculada com dados distribuídos usando (3.8), definindo $V=\overline{\mathbf{x}}, V[i i]=\overline{\mathbf{x}}[i i]$ (a média geral calculada localmente com apenas os objetos do $i$-ésimo sítio de dados) e $\phi[i i]=\psi[i i]=N_{i i}$. Agora, seja $V=V_{D K}$, $V[i i]=V_{K}[i i]$ o índice calculado localmente usando (2.80) no $i$-ésimo sítio de dados, i.e.:

$$
V_{K}[i i]=\frac{\sum_{i=1}^{k} \sum_{j=1}^{N_{i i}}\left(u[i i]_{i j}\right)^{m}\left\|\mathbf{x}[i i]_{j}-\mathbf{v}_{i}\right\|^{2}}{\min _{l \neq s}|| \mathbf{v}_{l}-\mathbf{v}_{s} \|^{2}}+\frac{\frac{1}{k} \sum_{i=1}^{k}\left\|\mathbf{v}_{i}-\overline{\mathbf{x}}\right\|^{2}}{\min _{l \neq s}|| \mathbf{v}_{l}-\mathbf{v}_{s} \|^{2}},
$$

$\phi[i i]=1, \psi[i i]=1 / P \mathrm{e}$

$$
\Delta=\frac{\frac{1}{k} \sum_{i=1}^{k}\left\|\mathbf{v}_{i}-\overline{\mathbf{x}}\right\|^{2}}{\min _{l \neq s}\left\|\mathbf{v}_{l}-\mathbf{v}_{s}\right\|^{2}}
$$

então, a versão distribuída do índice $K$ pode ser calculada usando (3.8), uma vez que (3.27) pode ser reescrita como:

$$
V_{D K}=\sum_{i i=1}^{P}\left[V_{K}[i i]-\frac{\frac{1}{k} \sum_{i=1}^{k}\left\|\mathbf{v}_{i}-\overline{\mathbf{x}}\right\|^{2}}{\min _{l \neq s}\left\|\mathbf{v}_{l}-\mathbf{v}_{s}\right\|^{2}}\right]+\frac{\frac{1}{k} \sum_{i=1}^{k}\left\|\mathbf{v}_{i}-\overline{\mathbf{x}}\right\|^{2}}{\min _{l \neq s}\left\|\mathbf{v}_{l}-\mathbf{v}_{s}\right\|^{2}}
$$




\subsubsection{DTSS: Distributed Tang-Sun-Sun}

O índice TSS em (2.82) pode ser reescrito como:

$$
V_{D T S S}=\frac{\sum_{i i=1}^{P} \sum_{i=1}^{k} \sum_{j=1}^{N_{i i}}\left(u[i i]_{i j}\right)^{m}\left\|\mathbf{x}[i i]_{j}-\mathbf{v}_{i}\right\|^{2}}{\min _{l \neq s}\left\|\mathbf{v}_{l}-\mathbf{v}_{s}\right\|^{2}+1 / k}+\frac{\frac{1}{k(k-1)} \sum_{i=1}^{k} \sum_{\substack{l=1 \\ l \neq i}}^{k}\left\|\mathbf{v}_{i}-\mathbf{v}_{l}\right\|^{2}}{\min _{l \neq s}\left\|\mathbf{v}_{l}-\mathbf{v}_{s}\right\|^{2}+1 / k}
$$

Este índice pode ser calculado com dados distribuídos usando (3.8), definindo $V=V_{D T S S}$,

$$
\Delta=\frac{\frac{1}{k(k-1)} \sum_{i=1}^{k} \sum_{\substack{l=1 \\ l \neq i}}^{k}\left\|\mathbf{v}_{i}-\mathbf{v}_{l}\right\|^{2}}{\min _{l \neq s}\left\|\mathbf{v}_{l}-\mathbf{v}_{s}\right\|^{2}+1 / k},
$$

$\phi[i i]=1, \psi[i i]=1 / P$ e $V[i i]=V_{T S S}[i i]$, em que $V_{T S S}[i i]$ é o índice calculado localmente usando (2.82) no $i$-ésimo sítio de dados, i.e:

$$
V_{T S S}[i i]=\frac{\sum_{i=1}^{k} \sum_{j=1}^{N_{i i}}\left(u[i i]_{i j}\right)^{m}\left\|\mathbf{x}[i i]_{j}-\mathbf{v}_{i}\right\|^{2}}{\min _{l \neq s}\left\|\mathbf{v}_{l}-\mathbf{v}_{s}\right\|^{2}+1 / k}+\frac{\frac{1}{k(k-1)} \sum_{i=1}^{k} \sum_{\substack{l=1 \\ l \neq i}}^{k}\left\|\mathbf{v}_{i}-\mathbf{v}_{l}\right\|^{2}}{\min _{l \neq s}\left\|\mathbf{v}_{l}-\mathbf{v}_{s}\right\|^{2}+1 / k}
$$

Dessa forma, a versão distribuída do índice TSS pode ser calculada como:

$$
V_{D T S S}=\sum_{i i=1}^{P}\left[V_{T S S}[i i]-\frac{\frac{1}{k(k-1)} \sum_{i=1}^{k} \sum_{\substack{l=1 \\ l \neq i}}^{k}\left\|\mathbf{v}_{i}-\mathbf{v}_{l}\right\|^{2}}{\min _{l \neq s}\left\|\mathbf{v}_{l}-\mathbf{v}_{s}\right\|^{2}+1 / k}\right]+\frac{\frac{1}{k(k-1)} \sum_{i=1}^{k} \sum_{\substack{l=1 \\ l \neq i}}^{k}\left\|\mathbf{v}_{i}-\mathbf{v}_{l}\right\|^{2}}{\min _{l \neq s}\left\|\mathbf{v}_{l}-\mathbf{v}_{s}\right\|^{2}+1 / k}
$$

\subsubsection{DFS: Distributed Fukuyama-Sugeno}

O índice FS em (2.83) pode ser reescrito como:

$$
V_{D F S}=\sum_{i i=1}^{P} \sum_{i=1}^{k} \sum_{j=1}^{N_{i i}}\left(u[i i]_{i j}\right)^{m}\left\|\mathbf{x}[i i]_{j}-\mathbf{v}_{i}\right\|^{2}-\sum_{i i=1}^{P} \sum_{i=1}^{k} \sum_{j=1}^{N_{i i}}\left(u[i i]_{i j}\right)^{m}\left\|\mathbf{v}_{i}-\overline{\mathbf{x}}\right\|^{2},
$$

em que $\overline{\mathbf{x}}=\frac{\sum_{i i=1}^{P} \sum_{j=1}^{N_{i i}} \mathbf{x}[i i]_{j}}{\sum_{i i=1}^{P} N_{i i}}$ é a média geral, que pode ser calculada com dados distribuídos usando (3.8), definindo $V=\overline{\mathbf{x}}, V[i i]=\overline{\mathbf{x}}[i i]$ (a média geral calculada localmente com apenas os objetos do $i i$-ésimo sítio de dados) e $\phi[i i]=\psi[i i]=N_{i i}$. Agora, seja $V=V_{D F S}$, $V[i i]=V_{F S}[i i]$ o índice calculado localmente usando (2.83) no $i i$-ésimo sítio de dados, i.e:

$$
V_{F S}[i i]=\sum_{i=1}^{k} \sum_{j=1}^{N_{i i}}\left(u[i i]_{i j}\right)^{m}\left\|\mathbf{x}[i i]_{j}-\mathbf{v}_{i}\right\|^{2}-\sum_{i=1}^{k} \sum_{j=1}^{N_{i i}}\left(u[i i]_{i j}\right)^{m}\left\|\mathbf{v}_{i}-\overline{\mathbf{x}}\right\|^{2}
$$

$\Delta=0, \phi[i i]=1$ e $\psi[i i]=1 / P$, então, a versão distribuída do índice FS pode ser calculada 
usando (3.8), uma vez que (3.33) pode ser reescrita como:

$$
V_{D F S}=\sum_{i i=1}^{P} V_{F S}[i i]
$$

\subsubsection{DFHV: Distributed Fuzzy Hypervolume}

O índice FHV em (2.84) necessita apenas das matrizes de covariância (globais). Tais matrizes e, consequentemente, a versão distribuída do índice FHV, podem ser calculadas seguindo os passos das Equações (3.9), (3.10) e (3.11).

\subsubsection{DAPD: Distributed Average Partition Density}

A versão distribuída do índice APD pode ser calculada de (2.85), obtendo os valores globais de $S_{i}$ e $\mathbf{F}_{i}$ de maneira distribuída. De maneira similar ao índice DFHV, na Seção 3.1.5.9, $\mathbf{F}_{i}$ pode ser calculado com dados distribuídos usando os passos das Equações (3.9)-(3.11). Analogamente, $S_{i}$ pode ser calculado usando (3.8) definindo $V=S_{i}$,

$$
V[i i]=S[i i]_{i}=\sum_{\mathbf{x}_{j} \in \mathbf{R}_{i}} u[i i]_{i j}
$$

$\phi[i i]=1$ e $\psi[i i]=1 / P$, de tal forma que

$$
S_{i}=\sum_{i i=1}^{P} \sum_{\mathbf{x}[i i]_{j} \in \mathbf{R}[i i]_{i}} u[i i]_{i j}=\sum_{i i=1}^{P} S[i i]_{i}
$$

em que $\mathbf{R}[i i]_{i}$ é o conjunto de objetos no $i i$-ésimo sítio de dados que estão em uma determinada região ao redor do protótipo $\mathbf{v}_{i}$, i.e., os objetos tais que $\left(\mathbf{x}[i i]_{j}-\mathbf{v}_{i}\right)^{T} \mathbf{F}_{i}^{-1}\left(\mathbf{x}[i i]_{j}-\mathbf{v}_{i}\right)<1$.

\subsubsection{DPD: Distributed Partition Density}

A versão distribuída do índice PD em (2.87) pode ser obtida como:

$$
V_{D P D}=\frac{\sum_{i=1}^{k} S_{i}}{V_{D F H V}},
$$

em que $V_{D F H V}$ é conforme descrito na Seção 3.1.5.9 e $S_{i}$ é dado por (3.36).

\subsubsection{DSCG: Distributed SCG}

O índice SCG em (2.88) requer as matrizes de covariância para que o termo GComp em (2.89) possa ser calculado. Conforme discutido anteriormente, as matrizes de covariância (globais) podem ser calculadas usando (3.9)-(3.11). O índice SCG também necessita das matrizes $\mathbf{S}_{B}$ em (2.91) para que o termo Sep em (2.90) possa ser calculado. O cálculo de 
$\mathbf{S}_{B}$ em um ambiente distribuído pode ser obtido usando (3.8), definindo $V=\mathbf{S}_{B}$,

$$
V[i i]=\mathbf{S}_{B}[i i]=\sum_{i=1}^{k} \sum_{j=1}^{N_{i i}}\left(u[i i]_{i j}\right)^{m}\left(\mathbf{v}_{i}-\overline{\mathbf{x}}\right)\left(\mathbf{v}_{i}-\overline{\mathbf{x}}\right)^{T}
$$

$\phi[i i]=1$ e $\psi[i i]=1 / P$, de tal forma que

$$
\mathbf{S}_{B}=\sum_{i i=1}^{P} \sum_{i=1}^{k} \sum_{j=1}^{N_{i i}}\left(u[i i]_{i j}\right)^{m}\left(\mathbf{v}_{i}-\overline{\mathbf{x}}\right)\left(\mathbf{v}_{i}-\overline{\mathbf{x}}\right)^{T} .
$$

Note que as equações necessitam da média geral $(\overline{\mathbf{x}})$, que também pode ser obtida usando (3.8), conforme descrito na Seção 3.1.5.6. Uma vez que tais termos são calculados, a versão distribuída do índice SCG, $V_{D S C G}$, pode ser obtida de (2.88).

\subsubsection{DPBMF: Distributed PBMF}

O índice PBMF pode ser reescrito como:

$$
V_{P B M F}=\left(\frac{1}{k} \cdot E_{1} \cdot D_{c}\right)^{2} \cdot\left(\frac{1}{J_{m}}\right)^{2} .
$$

Note que $E_{1}$ é uma constante e, portanto, não precisa ser calculado para realizar comparações relativas (Pakhira et al., 2005) ${ }^{5}$. O termo $\left(\frac{1}{k} \cdot E_{1} \cdot D_{c}\right)^{2}$ pode ser calculado no sítio central sem a necessidade de comunicação. O termo $J_{m}$ pode ser calculado usando (3.8) definindo $V=J_{m}, V[i i]=J_{m}[i i]$ (o valor calculado localmente no $i i$-ésimo sítio de dados), i.e: $J_{m}[i i]=\sum_{i=1}^{k} \sum_{j=1}^{N}\left(u[i i]_{i j}\right)^{m}\left\|\mathbf{x}[i i]_{j}-\mathbf{v}_{i}\right\|, \Delta=0, \phi[i i]=1$ e $\psi[i i]=1 / P$. Dessa forma, o índice DPBMF pode ser facilmente obtido (Modenesi et al., 2007).

\subsubsection{Análise de Complexidade - Comunicação}

Dado que a complexidade de tempo e espaço dos índices distribuídos seguem as análises de complexidades relatadas no Capítulo $2^{6}$, aqui será apresentada apenas a análise de complexidade de comunicação de cada algoritmo.

Seguindo o procedimento distribuído em (3.8), é necessário comunicar três variáveis: $V[i i], \phi[i i]$ e $\psi[i i]$. O tamanho da primeira depende do que se está comunicando. As outras $(\phi[i i]$ e $\psi[i i])$ são valores reais e requerem a comunicação de um único valor cada. Dessa forma, a complexidade de comunicação depende da definição de $V[i i]$.

Para o índice DFHV é necessário que as matrizes de covariância sejam calculadas, o que requer $O\left(k n^{2}\right)$. Para os índices DAPD e DPD é necessário calcular as matrizes de covariância e os valores de $S_{i}$ de cada grupo, o que requer $O\left(k n^{2}+k\right) \rightarrow O\left(k n^{2}\right)$. Para o

\footnotetext{
${ }^{5}$ Se desejado, este termo pode ser obtido para dados distribuídos calculando, primeiramente, a média geral $\overline{\mathbf{x}}$ usando (3.8) com $V=\overline{\mathbf{x}}, V[i i]=\overline{\mathbf{x}}[i i]$ (a média geral calculada localmente no $i i$-ésimo sítio de dados), $\Delta=0$ e $\phi[i i]=\psi[i i]=N_{i i}$, e então calculando $E_{1}$ usando (3.8) com $V=E_{1}, V[i i]=E_{1}[i i]$ (o valor calculado no $i$-ésimo sítio de dados), $\Delta=0, \phi[i i]=1$ e $\psi[i i]=1 / P$.

${ }^{6}$ Note que agora o número de objetos é dado por $N_{i i}$.
} 
índice DSCG é necessário calcular as matrizes de covariância globais, a média geral e $\mathbf{S}_{B}$, o que requer $O\left(k n^{2}+n+n^{2}\right) \rightarrow O\left(k n^{2}\right)$. Para o índice PBMF é necessário calcular a média geral, o que requer $O(n)$ e os protótipos globais, o que requer $O(k n)$. As complexidades de comunicação dos índices distribuídos estão sumarizadas na Tabela 3.3.

\begin{tabular}{lc||cc}
\hline Índice & $\begin{array}{c}\text { Complexidade de } \\
\text { Comunicação }\end{array}$ & Índice & $\begin{array}{c}\text { Complexidade de } \\
\text { Comunicação }\end{array}$ \\
\hline DXB & $O(P)$ & DFHV & $O\left(P k n^{2}\right)$ \\
DFSS & $O(P)$ & DAPD & $O\left(P\left(k n^{2}+k\right)\right) \rightarrow O\left(P k n^{2}\right)$ \\
DK & $O(P)$ & DPD & $O\left(P\left(k n^{2}+k\right)\right) \rightarrow O\left(P k n^{2}\right)$ \\
DTSS & $O(P)$ & DSCG & $O\left(P\left(k n^{2}+n^{2}\right)\right) \rightarrow O\left(P k n^{2}\right)$ \\
DFS & $O(P)$ & DPBMF & $O(P(n+k n)) \rightarrow O(P k n)$ \\
\hline
\end{tabular}

Tabela 3.3: Complexidade de Comunicação dos índices distribuídos: $P$ é o número de sítio de dados, $k$ é o número de grupos e $n$ é o número de atributos. 


\subsubsection{Estimação do Número de Grupos}

Uma vez que os algoritmos de agrupamento e os índices foram generalizados para trabalharem com dados distribuídos, o procedimento OMR usado para estimar o número de grupos descrito na Seção 2.6 também pode ser generalizado para trabalhar em cenários distribuídos. Mais especificamente, o usuário deve rodar um algoritmo de agrupamento distribuído e avaliar a qualidade de cada partição obtida por um índice de validade distribuído.

Tal abordagem, denotada por DOMR- $\mathcal{A}^{\mathcal{D}}$ (Distributed Ordered Multiple Runs of $\mathcal{A}^{\mathcal{D}}$ - em inglês), consiste no procedimento pelo qual o algoritmo de agrupamento desejado, $\mathcal{A}^{\mathcal{D}}$ (e.g. DFCM), executa $M$ vezes para cada número de grupos $k$ variando de um dado $k_{\min }$ (usualmente 2 ) até um dado $k_{\max }$. No final desse procedimento existirão $M \cdot\left(k_{\max }-\right.$ $\left.k_{\min }+1\right)$ partições que são avaliadas de acordo com determinado índice de validade, $\mathcal{I}^{\mathcal{D}}$. Este procedimento está detalhado no Algoritmo 28.

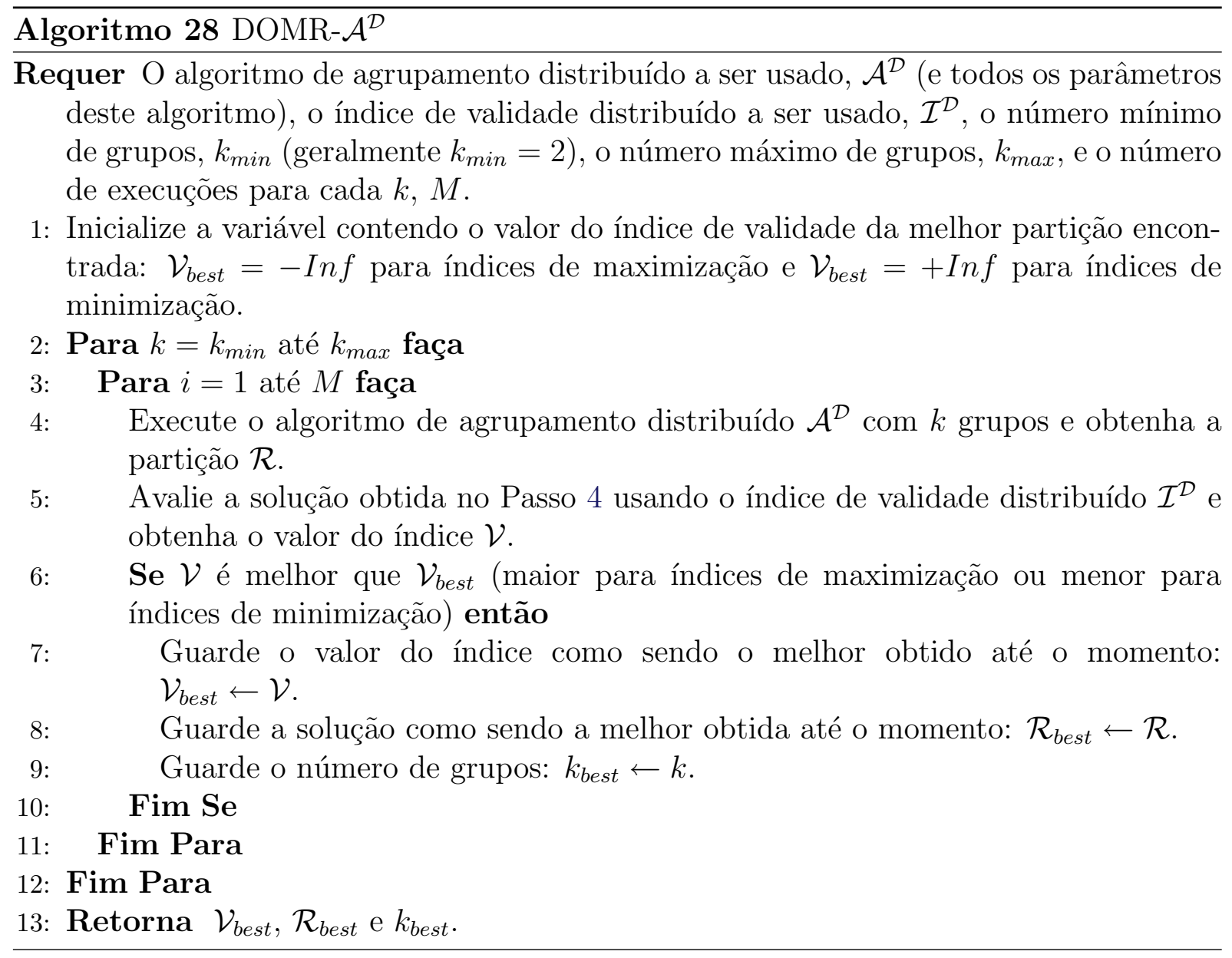




\subsubsection{Tabela de Cálculos para Cenários Distribuídos}

Os elementos usados nos algoritmos e índices de validade calculados com dados distribuídos usando (3.8) configurando $\Delta, \phi[i i]$ e $\psi[i i]$ estão ilustrados nas Tabelas 3.4 e $3.5^{7}$.

\begin{tabular}{|c|c|c|c|}
\hline & $\Delta$ & $\phi[i i]$ & $\psi[i i]$ \\
\hline $\mathbf{v}_{i}$ em $(2.10)$ & 0 & $\sum_{j=1}^{N_{i i}}\left(u[i i]_{i j}\right)^{m}$ & $\sum_{j=1}^{N_{i i}}\left(u[i i]_{i j}\right)^{m}$ \\
\hline $\mathbf{v}_{i}$ em $(2.38)$ & 0 & $\sum_{j=1}^{N_{i i}}\left(\left(u[i i]_{i j}\right)^{m}+\left(p[i i]_{i j}\right)^{\gamma}\right)$ & $\sum_{j=1}^{N_{i i}}\left(\left(u[i i]_{i j}\right)^{m}+\left(p[i i]_{i j}\right)^{\gamma}\right)$ \\
\hline $\mathbf{v}_{i}$ em $(2.42)$ & 0 & $\sum_{j=1}^{N_{i i}}\left(a\left(u[i i]_{i j}\right)^{m}+b\left(p[i i]_{i j}\right)^{\gamma}\right)$ & $\sum_{j=1}^{N_{i i}}\left(a\left(u[i i]_{i j}\right)^{m}+b\left(p[i i]_{i j}\right)^{\gamma}\right)$ \\
\hline $\mathbf{v}_{i}$ em $(2.59)$ & 0 & $\sum_{j=1}^{N_{i i}} w[i i]_{j}\left(u[i i]_{i j}\right)^{m}$ & $\sum_{j=1}^{N_{i i}} w[i i]_{j}\left(u[i i]_{i j}\right)^{m}$ \\
\hline$v_{i p} \mathrm{em}(2.56)$ & 0 & $\sum_{j=1}^{N_{i i}}\left(u[i i]_{i j}\right)^{m} I[i i]_{j p}$ & $\sum_{j=1}^{N_{i i}}\left(u[i i]_{i j}\right)^{m} I[i i]_{j p}$ \\
\hline $\mathbf{F}_{i}$ em $(2.13)$ & 0 & $\sum_{j=1}^{N_{i i}}\left(u[i i]_{i j}\right)^{m}$ & $\sum_{j=1}^{N_{i i}}\left(u[i i]_{i j}\right)^{m}$ \\
\hline$P_{i}$ em $(2.15)$ & 0 & $\sum_{j=1}^{N_{i i}} \sum_{c=1}^{k}\left(u[i i]_{c j}\right)^{m}$ & $\sum_{j=1}^{N_{i i}} \sum_{c=1}^{k}\left(u[i i]_{c j}\right)^{m}$ \\
\hline$\varphi_{i}$ em $(2.39)$ & 0 & 1 & $1 / \mathrm{P}$ \\
\hline$\eta_{i}$ em $(2.34)$ & 0 & $\sum_{j=1}^{N_{11}}\left(u[i i]_{i j}\right)^{m}$ & $\sum_{j=1}^{N_{11}}\left(u[i i]_{i j}\right)^{m}$ \\
\hline$e_{i, p, l}$ em (2.67) & 0 & 1 & $1 / \mathrm{P}$ \\
\hline \multicolumn{4}{|l|}{$J$ em $(2.6)$} \\
\hline \multicolumn{4}{|l|}{$(2.32),(2.35)$} \\
\hline $\mathrm{e}(2.40)$ & 0 & 1 & $1 / \mathrm{P}$ \\
\hline$\overline{\mathbf{x}}$ in $(2.81)$ & 0 & $N_{i i}$ & $N_{i i}$ \\
\hline$S_{i}$ em $(2.86)$ & 0 & 1 & $1 / \mathrm{P}$ \\
\hline $\mathbf{S}_{B}$ em $(2.91)$ & 0 & 1 & $1 / \mathrm{P}$ \\
\hline
\end{tabular}

Tabela 3.4: Cálculo dos elementos usados pelos algoritmos e índices distribuídos utilizando (3.8): $P$ é o número de sítios de dados, $k$ é o número de grupos, $m$ e $\gamma$ são coeficientes de fuzzificação, $N_{i i}$ é o número de objetos do $i i$-ésimo sítio de dados, $u[i i]_{i j}$ e $p[i i]_{i j}$ são as pertinências probabilísticas e possibilísticas, respectivamente, do $j$-ésimo objeto do $i i$ ésimo sítio de dados ao $i$-ésimo grupo, $I[i i]_{j p}$ é igual a 1 quando o $p$-ésimo atributo do $j$-ésimo objeto do $i i$-ésimo sítio de dados está presente (caso contrário é igual a 0 ) e $w[i i]_{j}$ é a quantidade de objetos (na matriz de dados original do $i i$-ésimo sítio de dados) que são representados pelo elemento $\mathbf{x}^{\prime}[i i]_{j}$.

\footnotetext{
${ }^{7}$ Note que, em todos os casos, $V[i i]$ e $V$ em (3.8) são os valores/índices calculados localmente no $i i$-ésimo sítio de dados e os valores/índices globais, respectivamente. Por este motivo e por simplicidade, eles estão omitidos nas tabelas.
} 


\begin{tabular}{|c|c|c|c|}
\hline & $\Delta$ & $\phi[i i]$ & $\psi[i i]$ \\
\hline $\mathrm{PC}$ & 0 & $N_{i i}$ & $N_{i i}$ \\
\hline$P E$ & 0 & $N_{i i}$ & $N_{i i}$ \\
\hline FS & 0 & 1 & $1 / P$ \\
\hline XB & 0 & $N_{i i}$ & $N_{i i}$ \\
\hline $\mathbf{K}$ & $\frac{\frac{1}{k} \sum_{i=1}^{k}\left\|\mathbf{v}_{i}-\overline{\mathbf{x}}\right\|^{2}}{\min _{l \neq s}\left\|\mathbf{v}_{l}-\mathbf{v}_{s}\right\|^{2}}$ & 1 & $1 / P$ \\
\hline TSS & $\frac{\frac{1}{k(k-1)} \sum_{i=1}^{k} \sum_{\substack{l=1 \\
l \neq i}}^{k}\left\|\mathbf{v}_{i}-\mathbf{v}_{l}\right\|^{2}}{\min _{l \neq s}\left\|\mathbf{v}_{l}-\mathbf{v}_{s}\right\|^{2}+1 / k}$ & 1 & $1 / P$ \\
\hline FSS & 0 & $\sum_{j=1}^{N_{i i}}\left(u_{p j}-u_{q j}\right)^{\alpha}$ & $\sum_{j=1}^{N_{i i}}\left(u_{p j}-u_{q j}\right)^{a}$ \\
\hline
\end{tabular}

Tabela 3.5: Cálculo dos índices distribuídos de acordo com (3.8).

\subsection{Agrupamento em Consenso}

Muitos algoritmos de agrupamento de dados foram propostos e investigados na literatura para encontrar estruturas em ambientes paralelos e distribuídos seguindo uma abordagem diferente da discutida anteriormente. Alguns desses algoritmos, denominados algoritmos de agrupamento colaborativos (collaborative clustering - em inglês), são executados em cada sítio de dados e, iterativamente, compartilham informações com os demais sítios com o objetivo de encontrar uma estrutura local usando informações dos demais sítios (Pedrycz, 2002, 2007; Pedrycz \& Rai, 2008; Coletta et al., 2012). Uma abordagem relacionada executa o algoritmo de agrupamento (centralizado) em cada sítio de dados e, após obter uma partição final, usa alguma espécie de combinação (ensemble em inglês) com o objetivo de melhorar os resultados obtidos em um sítio específico com informações fornecidas pelos demais sítios de dados (Dimitriadou et al., 2002; Strehl \& Ghosh, 2003; Agogino \& Tumer, 2006; Tumer \& Agogino, 2008). Essa última abordagem é algumas vezes denominada agrupamento em consenso (consensus clustering — em inglês) (Ghosh et al., 2002; Pedrycz \& Hirota, 2008; Vendramin et al., 2011).

Mais especificamente, no agrupamento de dados em consenso, cada sítio de dados encontra individualmente as estruturas existentes de acordo com seus objetos locais (e.g. aplica um determinado algoritmo de agrupamento aos seus objetos locais) e, então, as informações estruturais finais encontradas individualmente em cada sítio de dados são transferidas para os (e recebida dos) demais sítios. Por fim, em cada sítio de dados, a 
estrutura local encontrada anteriormente com os objetos locais é combinada com as informações provindas dos demais sítios e uma solução consenso é construída. Dessa forma, a solução anteriormente obtida no sítio local é apenas ajustada a partir de evidências advindas de outros sítios de dados. Nesse caso as soluções consenso finais obtidas em cada sítio de dados podem ser diferentes.

Nesta seção os algoritmos de agrupamento de dados em consenso desenvolvidos por Pedrycz \& Hirota (2008) são discutidos e aperfeiçoamentos propostos são apresentados em seguida.

\subsubsection{ACO-H: Agrupamento em Consenso Original - Caso Horizontal}

Pedrycz \& Hirota (2008) propuseram um framework para agrupamento de dados distribuído horizontalmente em que o objetivo é conciliar a estrutura obtida por objetos em uma base de dados com os resultados já disponíveis por outras bases de dados relacionadas.

Formalmente, seja $k_{i i}$ o número de grupos encontrados na forma de protótipos no $i i$ ésimo sítio de dados. O objetivo de um usuário no domínio do sítio de dados $\mathbf{X}[0]$ (sítio de dados local) ${ }^{8}$ é encontrar uma solução consenso $\tilde{\mathbf{U}}$, formada por $\tilde{k}$ grupos (valor que pode ser diferente do número de grupos existente no sítio local e nos demais sítios de dados) que reconcilie a partição fuzzy $\mathbf{U}[0]\left(k_{0} \times N_{0}\right)$, induzida do sítio local (base de dados $\left.\mathbf{X}[0]\right)$ e seus protótipos $(\mathbf{V}[0])$, com as matrizes de partição fuzzy $\mathbf{U}[0 \mid i i]\left(k_{i i} \times N_{0}\right)$, induzidas também dos dados locais de $\mathbf{X}[0]$, porém dos protótipos oriundos de outros sítios de dados $(\mathbf{V}[i i], i i=1, \ldots, P)^{9}$. Veja ilustração na Figura 3.4.

As matrizes de partição induzidas $\mathbf{U}[0 \mid i i](i i=1, \ldots, P)$ têm o mesmo número $\left(N_{0}\right)$ de colunas entre si (número de objetos da base de dados do sítio $\mathbf{X}[0]$ ) mas não necessariamente o mesmo número de linhas (número de protótipos dos grupos obtidos nos demais sítios de dados, $k_{i i}$ ). Adicionalmente, dado que permutações entre linhas dessas matrizes não afetam o agrupamento representado por elas, uma comparação direta entre as matrizes de partição induzidas (comparações entre as matrizes $\mathbf{U}[0], \mathbf{U}[0 \mid 1], \ldots, \mathbf{U}[0 \mid P]$ ) não pode ser efetuada mesmo quando o número de linhas dessas matrizes é o mesmo. Portanto, uma partição consenso $\tilde{\mathbf{U}}\left(\tilde{k} \times N_{0}\right)$ não pode ser obtida diretamente das matrizes de partição induzidas.

A matriz de proximidade apresentada em (Loia et al., 2007; Pedrycz \& Hirota, 2008) é uma das alternativas viáveis para lidar com o problema de comparar diretamente as matrizes de partição $\mathbf{U}[0 \mid i i]$ que podem apresentar número de grupos diferentes. Dada uma matriz de partição $\mathbf{U}[0 \mid i i]$, calcula-se a proximidade entre o $i$-ésimo e o $j$-ésimo objeto

\footnotetext{
${ }^{8}$ Note que agora existem $P+1$ sítios de dados. Entretanto, diferentemente da abordagem discutida na Seção 3.1, o objetivo deste algoritmo consiste em melhorar apenas a estrutura encontrada no sítio de dados local $\mathbf{X}[0]$. Dessa forma, a estrutura encontrada nos demais sítios $(\mathbf{X}[1], \ldots, \mathbf{X}[P])$ não é alterada, a menos que o algoritmo execute novamente em um dos demais sítios de dados (nesse caso tal sítio de dados passaria a ser interpretado como sítio de dados local).

${ }^{9}$ As matrizes de partição induzidas $(\mathbf{U}[0 \mid i i])$ são calculadas com os dados do sítio de dados local (X $\left.[0]\right)$ e os protótipos disponibilizados pelo $i i$-ésimo sítio $(\mathbf{V}[i i], i i=1, \ldots, P)$ usando o procedimento de cálculo da matriz de partição do algoritmo FCM (Equação (2.8)).
} 


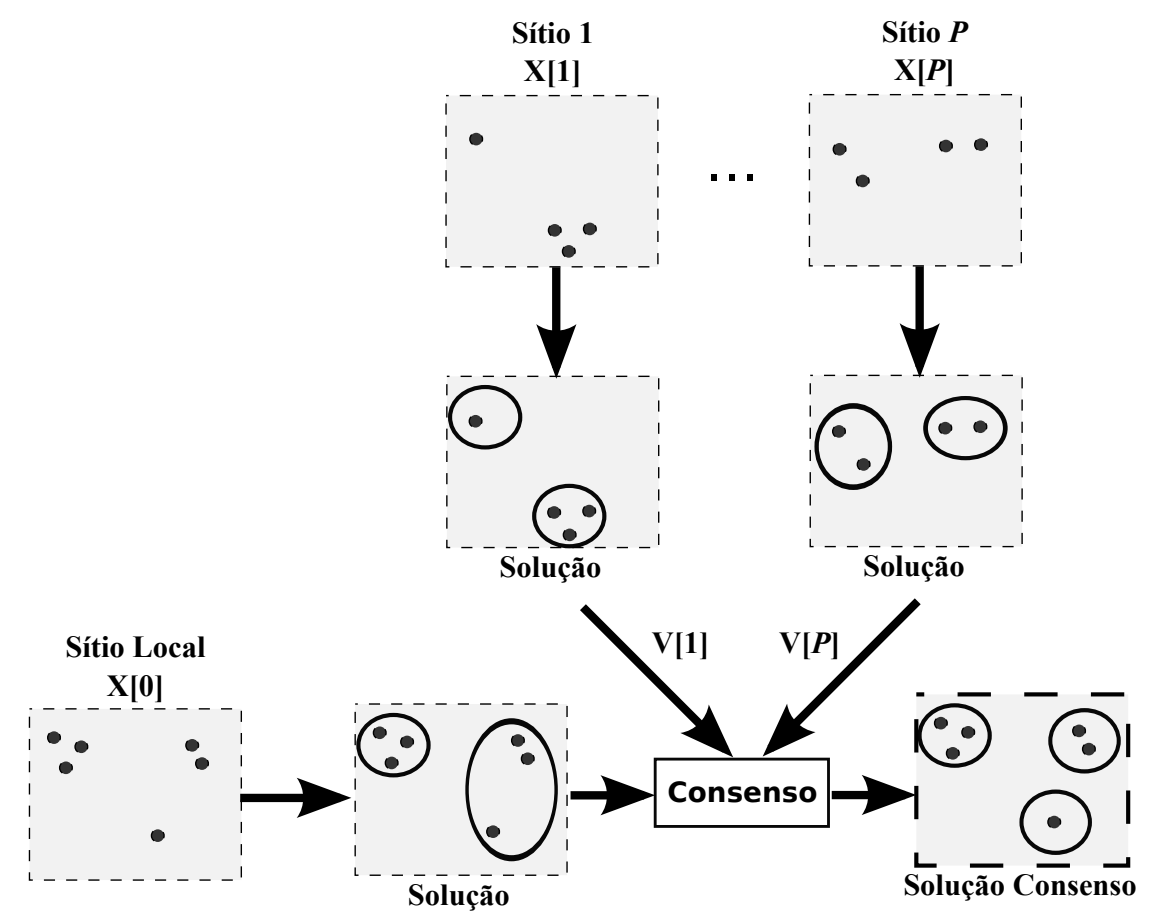

Figura 3.4: Agrupamento em Consenso - Caso Horizontal.

como:

$$
\operatorname{Prox}_{i j}[0 \mid i i]=\sum_{l=1}^{k_{i i}} \min \left(u_{l i}[0 \mid i i], u_{l j}[0 \mid i i]\right) .
$$

Ao calcular os valores de proximidade para todos os pares de objetos $\left(i, j=1, \ldots, N_{0}\right)$, obtém-se a matriz de proximidade, denotada por $\operatorname{Prox}[0 \mid i i](i i=1, \ldots, P)$. É importante notar que a matriz é formada por pares de objetos $\left(N_{0} \times N_{0}\right)$ e não depende explicitamente do número de grupos $\left(k_{i i}\right)$ formados para a base de dados do ii-ésimo sítio de dados. Note que, após a transformação de matrizes de partição induzidas $(\mathbf{U}[0 \mid i i])$ para matrizes de proximidade induzidas (Prox[0|ii]), obtém-se matrizes precisamente com a mesma dimensão, $N_{0} \times N_{0}$, que é determinada pelo número de objetos da base de dados na qual o consenso será aplicado (base de dados do sítio local - $\mathbf{X}[0]$ ). Deseja-se então

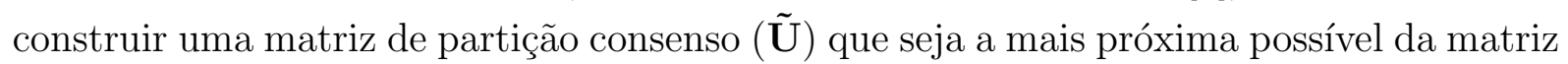
de partição local $(\mathbf{U}[0])$ e cuja matriz de proximidade, denotada por $\operatorname{Prox}(\tilde{\mathbf{U}})$, seja a mais próxima possível das matrizes de proximidade induzidas $(\operatorname{Prox}[0 \mid 1], \ldots, \operatorname{Prox}[0 \mid P])$. Formalmente, deseja-se minimizar (com respeito a $\tilde{\mathbf{U}}$ ) a seguinte equação (Pedrycz \& Hirota, 2008):

$$
H=\|\tilde{\mathbf{U}}-\mathbf{U}[0]\|^{2}+\alpha \sum_{i i=1}^{P}\|\operatorname{Prox}(\tilde{\mathbf{U}})-\operatorname{Prox}[0 \mid i i]\|^{2}
$$

Neste caso em particular, está assumido implicitamente no primeiro termo da Equação (3.42) que o número de grupos em $\tilde{\mathbf{U}}$ é o mesmo número de grupos em $\mathbf{U}[0]$, i.e., $\tilde{k}=k_{0}$. A constante $\alpha$ determina um balanceamento entre a confiança das estruturas 
encontradas no sítio de dados local $(\mathbf{U}[0])$ e as informações fornecidas pelos demais sítios de dados $(\mathbf{U}[0 \mid i i])$. O problema completo de otimização, com as restrições impostas para obter uma partição fuzzy probabilística, é dado por:

$$
\begin{array}{ll}
\min _{\tilde{\mathbf{U}}} & H=\|\tilde{\mathbf{U}}-\mathbf{U}[0]\|^{2}+\alpha \sum_{i i=1}^{P}\|\operatorname{Prox}(\tilde{\mathbf{U}})-\operatorname{Prox}[0 \mid i i]\|^{2} \\
\text { s.a. } & \sum_{l=1}^{\tilde{k}} \tilde{u}_{l j}=1, j=1, \ldots, N_{0} \\
& 0 \leq \tilde{u}_{l j} \leq 1, l=1, \ldots, \tilde{k}, j=1, \ldots, N_{0} .
\end{array}
$$

O algoritmo de consenso original, posposto por Pedrycz \& Hirota (2008) e denotado neste documento por ACO-H, consiste em um processo para minimizar a Equação (3.42). A minimização é realizada pelo método do gradiente descendente. Dessa forma, as derivadas parciais da função objetivo em (3.43) são utilizadas a cada passo do método e os elementos da matriz de partição são atualizados de acordo com uma taxa de aprendizagem $\eta$ determinada pelo usuário (Pedrycz \& Hirota, 2008). Entretanto, a atualização dos

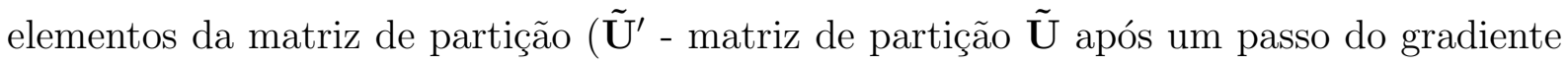
descendente) deve ser controlada para que as restrições do problema de otimização des-

crito em (3.43) sejam respeitadas. Para isso, cada coluna da matriz $\tilde{\mathbf{U}}^{\prime}$ é normalizada para somar 1 após um passo do processo de otimização do gradiente descendente. Assim, após a atualização e a normalização das pertinências em $\tilde{\mathbf{U}}^{\prime}$, substitui-se a matriz de partição $\tilde{\mathbf{U}}$ por $\tilde{\mathbf{U}}^{\prime}$ e o próximo passo do gradiente descendente é executado.

\subsubsection{ACM-H: Agrupamento em Consenso Modificado - Caso Horizontal}

O problema de otimização para resolver o modelo descrito na Seção 3.2.1 pode ser formulado e solucionado de maneira mais rigorosa, tanto na perspectiva conceitual (modelo) quanto numérica (algoritmo). Veja que a maneira pela qual as proximidades são calculadas na Equação (3.41) faz com que o processo utilizado para encontrar a solução seja mais complexo, já que a descontinuidade da função min(.) impõe dificuldades adicionais ao algoritmo de otimização. Visando contornar este problema, é proposto neste trabalho o uso de uma definição alternativa para a proximidade entre o $i$-ésimo e o $j$-ésimo objeto, dada por:

$$
\operatorname{Prox}_{i j}[0 \mid i i]=1-\frac{1}{2} \sum_{l=1}^{k_{i i}}\left(u_{l i}[0 \mid i i]-u_{l j}[0 \mid i i]\right)^{2},
$$

de tal forma que $\operatorname{Prox}_{i j}[0 \mid i i] \in[0,1]$.

Diferentes sítios de dados podem prover maior/menor confiança ou interesse do usuário. O usuário pode conhecer, a priori, os sítios de dados mais ou menos importantes sob a perspectiva do sítio local $(\mathbf{X}[0])$ em que o processamento está sendo realizado. Nesse sentido, é interessante que o processo de otimização leve em conta tais prioridades. Para 
tanto, pode-se reescrever a Equação (3.42) como:

$$
H=\sum_{i i=0}^{P} \alpha_{i i} \| \operatorname{Prox}(\tilde{\mathbf{U}})-\operatorname{Prox}[0 \mid i i]||^{2},
$$

em que $\alpha_{i i} \in[0,1]$ é o peso do $i i$-ésimo sítio de dados. Note que, modificando o primeiro termo da Equação (3.42) para matriz de proximidade, a equação torna-se "homogênea", fazendo com que a interpretação seja mais clara (trata-se de termos da mesma grandeza) e a escolha dos pesos $\alpha$ não seja mais um balanceamento entre duas grandezas distintas. Note, também, que a formulação descrita pela Equação (3.45) permite que o usuário

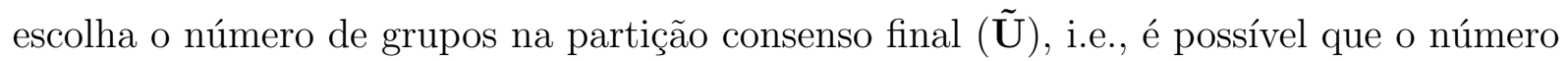
de grupos $\tilde{k}$ na partição consenso seja diferente do número de grupos $k_{0}$ do sítio de dados local e do número de grupos $k_{i i}$ dos demais sítios de dados. A escolha de um número de grupos diferente pode ser um ponto muito importante, pois é possível que as informações providas pelos outros sítios de dados indiquem que existem mais (ou menos) grupos do que o encontrado sem essas informações.

O algoritmo de consenso modificado, proposto neste documento e denotado por ACM-H, consiste em um processo para minimizar a Equação (3.45). O problema de otimização é dado por:

$$
\begin{array}{ll}
\min _{\tilde{\mathbf{U}}} & H=\sum_{i i=0}^{P} \alpha_{i i} \sum_{i=1}^{N_{0}} \sum_{j=1}^{N_{0}}\left(\operatorname{Prox}_{i j}(\tilde{\mathbf{U}})-\operatorname{Prox}_{i j}[0 \mid i i]\right)^{2} \\
\text { s.a. } & \sum_{l=1}^{\tilde{k}} \tilde{u}_{l j}=1, j=1, \ldots, N_{0} \\
& 0 \leq \tilde{u}_{l j} \leq 1, l=1, \ldots, \tilde{k}, j=1, \ldots, N_{0} .
\end{array}
$$

Dado que a matriz de proximidade é simétrica, então minimizar $H$ em (3.46) é equivalente a minimizar a seguinte versão simétrica:

$$
H=\sum_{i i=0}^{P} \alpha_{i i} \sum_{i=1}^{j-1} \sum_{j=2}^{N_{0}}\left(\operatorname{Prox}_{i j}(\tilde{\mathbf{U}})-\operatorname{Prox}_{i j}[0 \mid i i]\right)^{2}
$$

Para o uso de um método de otimização baseado em gradiente é necessário o cálculo do gradiente de $H$ com respeito às entradas correspondentes da matriz de partição, obtido a partir de (3.47) e (3.44) como: 


$$
\begin{aligned}
\frac{\partial H}{\partial \tilde{u}_{s t}} & =\sum_{i i=0}^{P} \alpha_{i i} \sum_{i=1}^{N_{0}} \frac{\partial\left(\operatorname{Prox}_{i t}(\tilde{\mathbf{U}})-\operatorname{Prox}_{i t}[0 \mid i i]\right)^{2}}{\partial \tilde{u}_{s t}}= \\
& =2 \sum_{i i=0}^{P} \alpha_{i i} \sum_{i=1}^{N_{0}}\left(\operatorname{Prox}_{i t}(\tilde{\mathbf{U}})-\operatorname{Prox}_{i t}[0 \mid i i]\right) \frac{\partial\left(\operatorname{Prox}_{i t}(\tilde{\mathbf{U}})\right)}{\partial \tilde{u}_{s t}}= \\
& =2 \sum_{i i=0}^{P} \alpha_{i i} \sum_{i=1}^{N_{0}}\left(\operatorname{Prox}_{i t}(\tilde{\mathbf{U}})-\operatorname{Prox}_{i t}[0 \mid i i]\right)\left(\tilde{u}_{s t}-\tilde{u}_{s i}\right) .
\end{aligned}
$$

Note, novamente, que a atualização dos elementos da matriz de partição deve ser controlada para que as restrições do problema de otimização descrito na Equação (3.46) sejam respeitadas. Portanto, cada coluna da matriz Ũ deve "somar" 1 após um passo do processo de otimização. O procedimento adotado no algoritmo de consenso original, ACO-H, é prejudicial ao processo de otimização, uma vez que altera o resultado (matriz de partição $\tilde{\mathbf{U}}$ ) encontrado a cada passo do processo de otimização, i.e., modifica a "direção" obtida pelo processo de otimização visando respeitar as restrições do Problema (3.43).

Uma abordagem mais apropriada para evitar esse problema consiste em utilizar um método de otimização não-linear com restrições, i.e., um método de otimização que minimize a função objetivo respeitando as restrições impostas internamente ao método. Por exemplo, uma das abordagens utilizadas na literatura para otimizar funções não lineares com restrições consiste no método dos pontos interiores (Byrd et al., 2000). Dessa forma, a versão modificada do processo de consenso (ACO-H), denotada por ACM-H, utiliza as derivadas parciais descritas na Equação (3.48) com o método dos pontos interiores como algoritmo de otimização para solucionar o problema com restrições em (3.46).

\subsubsection{Estimação do Número de Grupos}

Um dos parâmetros necessários para a execução do processo de consenso descrito nas Seções 3.2.1 e 3.2.2 é o número de grupos, $\tilde{k}$, contido na matriz de partição consenso, $\tilde{\mathbf{U}}$. O número de grupos, $k_{i i}$, também deve ser conhecido em cada sítio de dados individualmente. Entretanto, o número mais natural de grupos frequentemente não é conhecido a priori pelo usuário. O procedimento OMR (Seção 2.6) pode ser facilmente aplicado a cada sítio de dados individualmente - executando o algoritmo de agrupamento várias vezes com número de grupos, $k_{i i}$, variado e então selecionando a melhor partição de acordo com determinado índice de validade relativo (não distribuído). Tais partições serão, então, enviadas ao sítio de dados local para a execução do algoritmo em consenso. Contudo, tal procedimento não é apropriado para encontrar a solução consenso no sítio de dados local, pois o uso de um índice de validade distribuído (Seção 3.1.5) presume que a partição é obtida utilizando todos os dados de todos os sítios de dados. Dado que o agrupamento em consenso consiste em apenas melhorar a solução local utilizando informações fornecidas pelos demais sítios de dados, uma possível alternativa consiste em executar o processo de 
consenso várias vezes com número de grupos, $\tilde{k}$, variado e escolher a solução que apresenta o menor valor da função objetivo (Equação (3.47)), ou seja, usar a própria função objetivo do modelo de otimização ao invés de um índice de validade relativo distribuído.

Em resumo, na abordagem denotada aqui por OMR-ACM-H, o procedimento OMR é executado individualmente em cada sítio de dados separadamente, usando um índice de validade relativo (não distribuído) para escolher a melhor partição local. Assim, uma única partição é obtida em cada sítio de dados. Os protótipos obtidos em cada sítio de dados $(\mathbf{V}[i i])$ são transferidos para o sítio de dados local (no qual o consenso será aplicado). Com os protótipos de cada sítio de dados, as matrizes de partições induzidas são calculadas ( $\mathbf{U}[0 \mid i i])$. Cada matriz de partição induzida é, então, transformada em uma matriz de proximidade induzida (Prox $[0 \mid i i]$ ). Finalmente, o processo de consenso (ACMH) executa várias vezes com número de grupos, $\tilde{k}$, variado e a partição que apresenta o menor valor da função objetivo (3.47) é selecionada como a solução para o processo de consenso. Os passos para o procedimento OMR-ACM-H são descritos no Algoritmo 29.

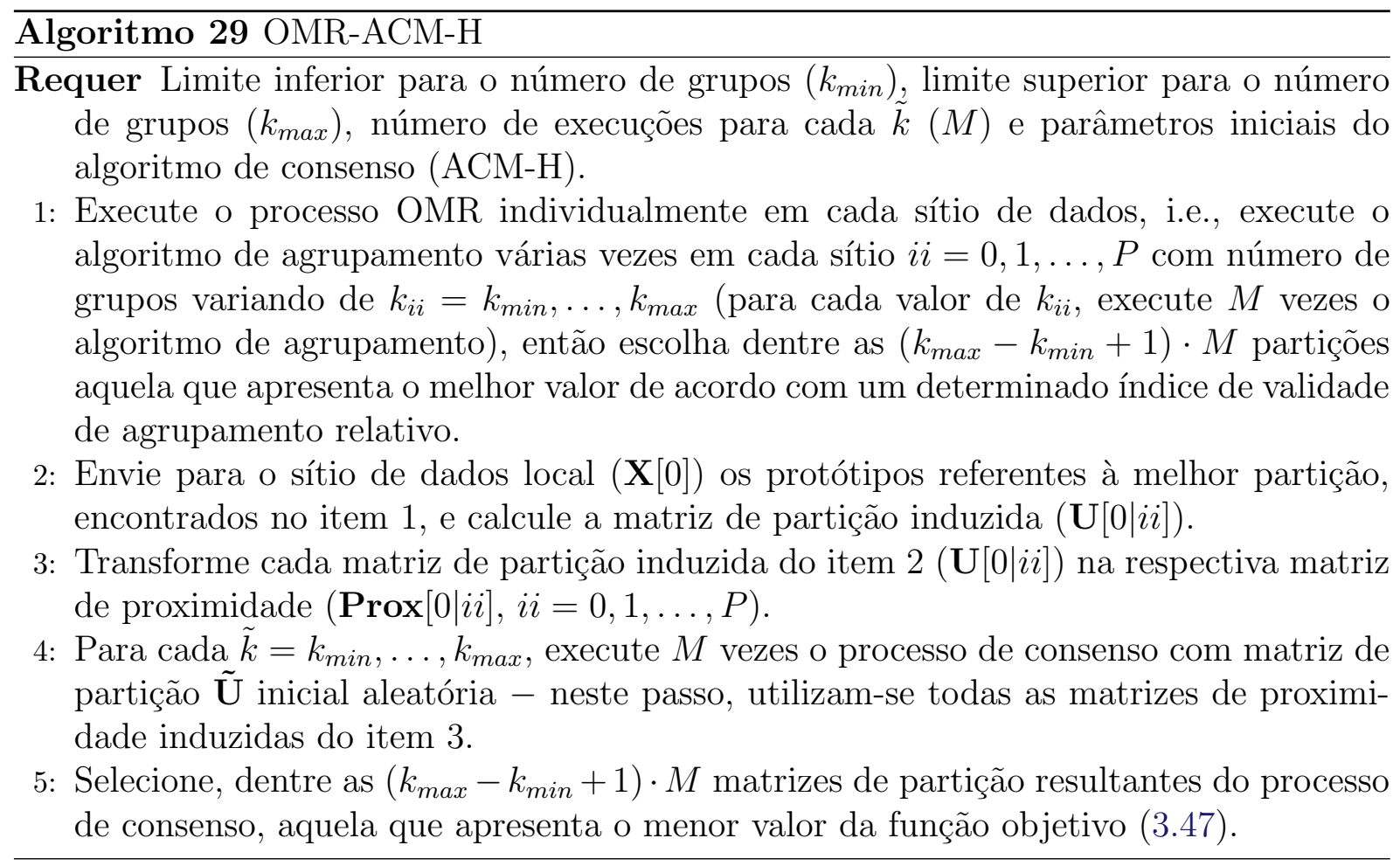

\subsubsection{Eliminação de Grupos Não Representativos}

O procedimento para estimar o número de grupos no processo de consenso, discutido na seção anterior, pode resultar em uma partição com grupos não representativos. Esses grupos não representam nenhum objeto, i.e., nenhum objeto $\left(\mathbf{x}_{i}\right)$ da base de dados apresenta pertinência com maior valor para o grupo não representativo $\left(\mathbf{C}_{j}\right)$. Tome, por exemplo, a matriz de partição com as pertinências de 7 objetos em 4 grupos, ilustrada na Tabela 3.6. 


$\tilde{\mathbf{U}}=$\begin{tabular}{|c|c|c|c|c|c|c|c|c|}
\hline & & \multicolumn{7}{|c|}{ Objetos } \\
\hline & & $\mathbf{1}$ & $\mathbf{2}$ & $\mathbf{3}$ & $\mathbf{4}$ & $\mathbf{5}$ & $\mathbf{6}$ & $\mathbf{7}$ \\
\hline \multirow{2}{*}{} & $\mathbf{1}$ & $\mathbf{0 . 6 9}$ & $\mathbf{0 . 6 7}$ & $\mathbf{0 . 5 7}$ & 0.19 & 0.08 & 0.14 & 0.08 \\
${ } }$ & $\mathbf{2}$ & 0.23 & 0.08 & 0.25 & $\mathbf{0 . 5 6}$ & $\mathbf{0 . 6 7}$ & 0.21 & 0.17 \\
& $\mathbf{3}$ & 0.08 & 0.08 & 0.16 & 0.19 & 0.08 & $\mathbf{0 . 6 3}$ & $\mathbf{0 . 6 7}$ \\
& $\mathbf{4}$ & 0.00 & 0.17 & 0.02 & 0.06 & 0.17 & 0.02 & 0.08 \\
\hline \hline
\end{tabular}

Tabela 3.6: Matriz de partição de 7 objetos e 4 grupos.

\begin{tabular}{|c|c|c|c|c|c|c|c|c|}
\hline \multirow{5}{*}{$\dot{z}$} & & \multicolumn{7}{|c|}{ Objetos } \\
\hline & & 1 & 2 & 3 & 4 & 5 & 6 & 7 \\
\hline & 1 & 0.69 & 0.81 & 0.58 & 0.20 & 0.10 & 0.14 & 0.09 \\
\hline & 2 & 0.23 & 0.10 & 0.26 & 0.60 & 0.80 & 0.21 & 0.18 \\
\hline & 3 & 0.08 & 0.10 & 0.16 & 0.20 & 0.10 & 0.65 & 0.73 \\
\hline
\end{tabular}

Tabela 3.7: Matriz de partição — grupo não representativo foi eliminado.

Note que o quarto grupo ilustrado na Tabela 3.6 consiste em um grupo não representativo. De fato, nenhum dos 7 objetos nessa base de dados apresenta o quarto grupo como grupo mais relevante. Nesses casos, o grupo não representativo pode ser eliminado e os valores de pertinência de cada objeto a esse grupo também podem ser eliminados. Ao eliminar todos os valores de pertinência do grupo não representativo, é necessário que as restrições impostas pelas Equações (2.1) e (2.2) sejam mantidas. Uma alternativa simples consiste em normalizar cada coluna da matriz para somar 1. Para tanto, basta dividir cada valor individual pela soma das pertinências do respectivo objeto a todos os grupos. Isso garante que a soma das pertinências de cada objeto em relação a todos os demais grupos é unitária. O resultado desse procedimento aplicado à matriz de partição ilustrada na Tabela 3.6 é ilustrado na Tabela 3.7. Uma alternativa mais interessante conceitualmente é, após remover o grupo não representativo, fornecer a matriz resultante de volta ao processo de otimização e executar algumas iterações ou até que o processo convirja novamente.

\subsection{Dados Distribuídos Verticalmente}

A abordagem discutida anteriormente, na Seção 3.2, também pode ser utilizada para trabalhar com dados distribuídos verticalmente (sítios de dados com os mesmos objetos, porém descritos por atributos diferentes). Dada uma coleção de objetos $\mathbf{X}$ em que seus atributos estão naturalmente descritos em $P+1$ diferentes espaços de características, $\mathbf{X}[0], \mathbf{X}[1], \ldots, \mathbf{X}[P]$ (cada espaço pode possuir diferentes atributos dos dados), a descoberta da estrutura contida em $\mathbf{X}$ pode ser obtida explorando duas abordagens diferentes (Loia et al., 2007):

(a) Concatenar os diferentes espaços de atributos dos objetos, $\mathbf{X}[0], \mathbf{X}[1], \ldots, \mathbf{X}[P]$, em 
uma única matriz dos dados $\mathbf{X}$. Existem algumas vantagens e desvantagens nesta abordagem. Dada sua familiaridade e aceitação geral, o uso de um único espaço de atributos pode ser benéfico. Em contrapartida, não é claro como cada subespaço $\mathbf{X}[i i]$ contribui para a formação do espaço como um todo $\mathbf{X}$. Alguns desses subespaços poderiam apresentar contribuições limitadas para o processo de agrupamento. Por exemplo, os subespaços podem ser constituídos de atributos que não são diretamente comensuráveis, tais como atributos fora de escala ou a existência de diferentes tipos de atributos (e.g. numéricos e categóricos). Nesses casos o uso de uma função de distância com pesos diferentes para as coordenadas individuais de cada subespaço poderia introduzir distorções nas relações existentes entre os dados.

(b) Tratar os objetos separadamente aceitando diferenças conceituais significativas entre os atributos, aplicar o processo de agrupamento em cada subespaço $(\mathbf{X}[0], \mathbf{X}[1], \ldots, \mathbf{X}[P])$ e, então, conciliar os resultados obtidos em um consenso final. Note que as funções de distância e as relações obtidas entre os objetos em cada subespaço não necessitam ser da mesma natureza.

Nesta seção é apresentado um algoritmo de agrupamento que adota a segunda abordagem, e também é proposto um aperfeiçoamento com melhorias deste algoritmo.

\subsubsection{ACO-V: Agrupamento em Consenso Original - Caso Vertical}

No agrupamento de dados vertical (dados distribuídos verticalmente), os diferentes sítios de dados apresentam os mesmos objetos porém atributos diferentes (nesses casos podem ser interpretados como diferentes subespaços). Como os objetos estão dispostos em diferentes subespaços, a comunicação entre os sítios de dados com matrizes de partição é mais adequada do que o uso de protótipos ${ }^{10}$. Denotam-se as matrizes de partições obtidas para cada subespaço $\mathbf{X}[0], \mathbf{X}[1], \ldots, \mathbf{X}[P]$ como $\mathbf{U}[0], \mathbf{U}[1], \ldots, \mathbf{U}[P]$, respectivamente. É importante notar que o número de grupos pode ser diferente em subespaços diferentes. Dado que o número de grupos em cada subespaço, $k_{0}, k_{1}, \ldots, k_{p}$, pode ser diferente, uma comparação direta entre as matrizes de partição $\mathbf{U}[0], \mathbf{U}[1], \ldots, \mathbf{U}[P]$ não é possível. Para lidar com esse problema é necessário utilizar um mecanismo que seja independente do número de grupos. Novamente, a matriz de proximidade (Prox $[i i]$ ) calculada usando a Equação (3.41) pode ser usada para contornar esse problema.

Para formar uma estrutura que seja "global" e que concilie todas as subestruturas

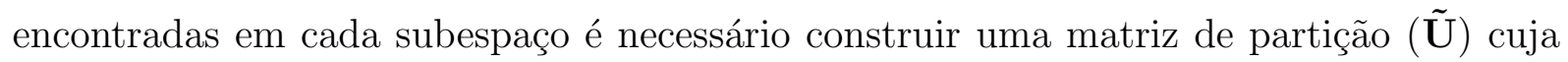
matriz de proximidade, dada por $\operatorname{Prox}(\tilde{\mathbf{U}})$, seja a mais próxima possível das matrizes de proximidade em cada subespaço $(\operatorname{Prox}[0], \operatorname{Prox}[1], \ldots, \operatorname{Prox}[p])$. Formalmente, deseja-se

\footnotetext{
${ }^{10}$ Note que, como os sítios de dados possuem atributos diferentes, os protótipos em um espaço podem não ser definidos nos demais espaços.
} 
minimizar (com respeito a Ũ) a seguinte equação (Loia et al., 2007):

$$
V=\|\operatorname{Prox}(\tilde{\mathbf{U}})-\operatorname{Prox}[0]\|^{2}+\left.\|\operatorname{Prox}(\tilde{\mathbf{U}})-\operatorname{Prox}[1]\|\right|^{2}+\cdots+\|\operatorname{Prox}(\tilde{\mathbf{U}})-\operatorname{Prox}[P]\|^{2}
$$

É importante notar que o uso exclusivo de matrizes de proximidade em (3.49) relaxa a necessidade de adotar o mesmo número de grupos em cada sítio e na estrutura final, o que não ocorria com o algoritmo horizontal descrito na Seção 3.2.1 com relação ao número de grupos no sítio local. O problema de otimização é dado por:

$$
\begin{array}{ll}
\min _{\tilde{\mathbf{U}}} & V=\sum_{i i=0}^{P} \sum_{i=1}^{N} \sum_{j>i}^{N}\left(\operatorname{Prox}_{i j}(\tilde{\mathbf{U}})-\operatorname{Prox}_{i j}[i i]\right)^{2} \\
\text { s.a. } & \sum^{\tilde{k}} \tilde{u}_{l j}=1, j=1, \ldots, N \\
& 0 \leq \tilde{u}_{l j} \leq 1, l=1, \ldots, \tilde{k}, j=1, \ldots, N .
\end{array}
$$

O algoritmo de consenso original para o caso vertical, posposto por Loia et al. (2007) e denotado neste documento por ACO-V, consiste em um processo para minimizar a Equação (3.49). A minimização é realizada pelo método do gradiente descendente. Dessa forma, as derivadas parciais da função objetivo em (3.50) são utilizadas a cada passo do método e os elementos da matriz de partição são atualizados de acordo com uma taxa de aprendizagem $\eta$ determinada pelo usuário (Loia et al., 2007). Entretanto, a atualização dos elementos da matriz de partição ( $\tilde{\mathbf{U}}^{\prime}$ - matriz de partição $\tilde{\mathbf{U}}$ após um passo do gradiente descendente) deve ser controlada para que as restrições do problema de otimização descrito em (3.50) sejam respeitadas. Para isso, cada coluna da matriz $\tilde{U}^{\prime}$ é normalizada para somar 1 após um passo do processo de otimização do gradiente descendente. Assim, após a atualização e a normalização das pertinências em $\tilde{\mathbf{U}}^{\prime}$, substitui-se a matriz de partição $\tilde{\mathbf{U}}$ por $\tilde{\mathbf{U}}^{\prime}$ e o próximo passo do gradiente descendente é executado.

\subsubsection{ACM-V: Agrupamento em Consenso Modificado - Caso Vertical}

Novamente, a abordagem discutida anteriormente na Seção 3.2.2, para dados distribuídos horizontalmente, também pode ser utilizada para trabalhar com dados distribuídos verticalmente. Formalmente, seguindo a mesma notação adotada na Seção 3.3.1, é proposto neste documento minimizar (com respeito a $\tilde{\mathbf{U}}$ ) a seguinte função:

$$
V=\sum_{i i=0}^{P} \alpha_{i i}\|\operatorname{Prox}(\tilde{\mathbf{U}})-\operatorname{Prox}[i i]\|^{2} .
$$

Note que nessa formulação existem constantes $\left(\alpha_{i i}\right)$ opcionais que determinam a importância que o $i$-ésimo subespaço representa. Assim como no algoritmo ACM-H (Seção 3.2 .2 ), visando contornar o problema causado pela descontinuidade da função $\min ($.$) em$ 
(3.41), agora as matrizes de proximidades são calculadas usando a Equação (3.44). O algoritmo de consenso modificado para o caso vertical, proposto neste documento e denotado por ACM-V, consiste em um processo para minimizar a Equação (3.51). O problema de otimização é dado por:

$$
\begin{array}{ll}
\min _{\mathbf{U}} & V=\sum_{i i=0}^{P} \alpha_{i i} \sum_{i=1}^{N} \sum_{j=1}^{N}\left(\operatorname{Prox}_{i j}(\tilde{\mathbf{U}})-\operatorname{Prox}_{i j}[i i]\right)^{2} \\
\text { s.a. } & \sum_{l=1}^{\tilde{k}} \tilde{u}_{l j}=1, j=1, \ldots, N \\
& 0 \leq \tilde{u}_{l j} \leq 1, l=1, \ldots, \tilde{k}, j=1, \ldots, N .
\end{array}
$$

Dado que a matriz de proximidade é simétrica, então minimizar $V$ em (3.52) é equivalente a minimizar a seguinte versão simétrica:

$$
V=\sum_{i i=0}^{P} \alpha_{i i} \sum_{i=1}^{j-1} \sum_{j=2}^{N}\left(\operatorname{Prox}_{i j}(\tilde{\mathbf{U}})-\operatorname{Prox}_{i j}[i i]\right)^{2} .
$$

Para o uso de um método de otimização baseado em gradiente é necessário o cálculo do gradiente de $V$ com respeito às entradas correspondentes da matriz de partição, obtido a partir de (3.53) e (3.44) como:

$$
\begin{aligned}
\frac{\partial V}{\partial \tilde{u}_{s t}} & =\sum_{i i=0}^{P} \alpha_{i i} \sum_{i=1}^{N} \frac{\partial\left(\operatorname{Prox}_{i t}(\tilde{\mathbf{U}})-\operatorname{Prox}_{i t}[i i]\right)^{2}}{\partial \tilde{u}_{s t}}= \\
& =2 \sum_{i i=0}^{P} \alpha_{i i} \sum_{i=1}^{N}\left(\operatorname{Prox}_{i t}(\tilde{\mathbf{U}})-\operatorname{Prox}_{i t}[i i]\right) \frac{\partial\left(\operatorname{Prox}_{i t}(\tilde{\mathbf{U}})\right)}{\partial \tilde{u}_{s t}}= \\
& =2 \sum_{i i=0}^{P} \alpha_{i i} \sum_{i=1}^{N}\left(\operatorname{Prox}_{i t}(\tilde{\mathbf{U}})-\operatorname{Prox}_{i t}[i i]\right)\left(\tilde{u}_{s t}-\tilde{u}_{s i}\right) .
\end{aligned}
$$

Note, novamente, que a atualização dos elementos da matriz de partição deve ser controlada para que as restrições do problema de otimização descrito em (3.52) sejam respeitadas. Portanto, cada coluna da matriz $\tilde{\mathbf{U}}$ deve "somar" 1 após um passo do processo de otimização. O procedimento adotado no algoritmo de consenso original, ACO-V, é prejudicial ao processo de otimização, uma vez que altera o resultado (matriz de partição Ü) encontrado a cada passo do processo de otimização, i.e., modifica a "direção" obtida pelo processo de otimização visando respeitar as restrições do Problema (3.52).

Novamente, uma abordagem mais apropriada para evitar esse problema consiste em utilizar um método de otimização não-linear com restrições, i.e., um método de otimização que minimiza a função objetivo já respeitando as restrições que são impostas internamente ao método. A versão modificada do processo de consenso (ACO-V), denotada por ACM-V, utiliza as derivadas parciais descritas na Equação (3.54) com o método dos pontos interiores como algoritmo de otimização. 


\subsubsection{Estimação do Número de Grupos}

Analogamente ao caso horizontal, na abordagem denotada aqui por OMR-ACM-V, o procedimento OMR é executado em cada sítio de dados separadamente, usando um índice de validade relativo (não distribuído) para escolher a melhor partição. Assim, uma única partição é obtida em cada sítio de dados. As matrizes de partições de cada sítio de dados $(\mathbf{U}[i i])$ são transformadas em matrizes de proximidade (Prox $[i i])$. Finalmente, o processo de consenso (ACM-V) executa várias vezes com número de grupos, $\tilde{k}$, variado e a solução que apresenta o menor valor da função objetivo (3.52) é selecionada como a solução para o processo de consenso. Em resumo, os passos para o procedimento OMR-ACM-V são descritos no Algoritmo 30. Após obter a partição consenso final, é importante também eliminar possíveis grupos não representativos (Seção 3.2.4).

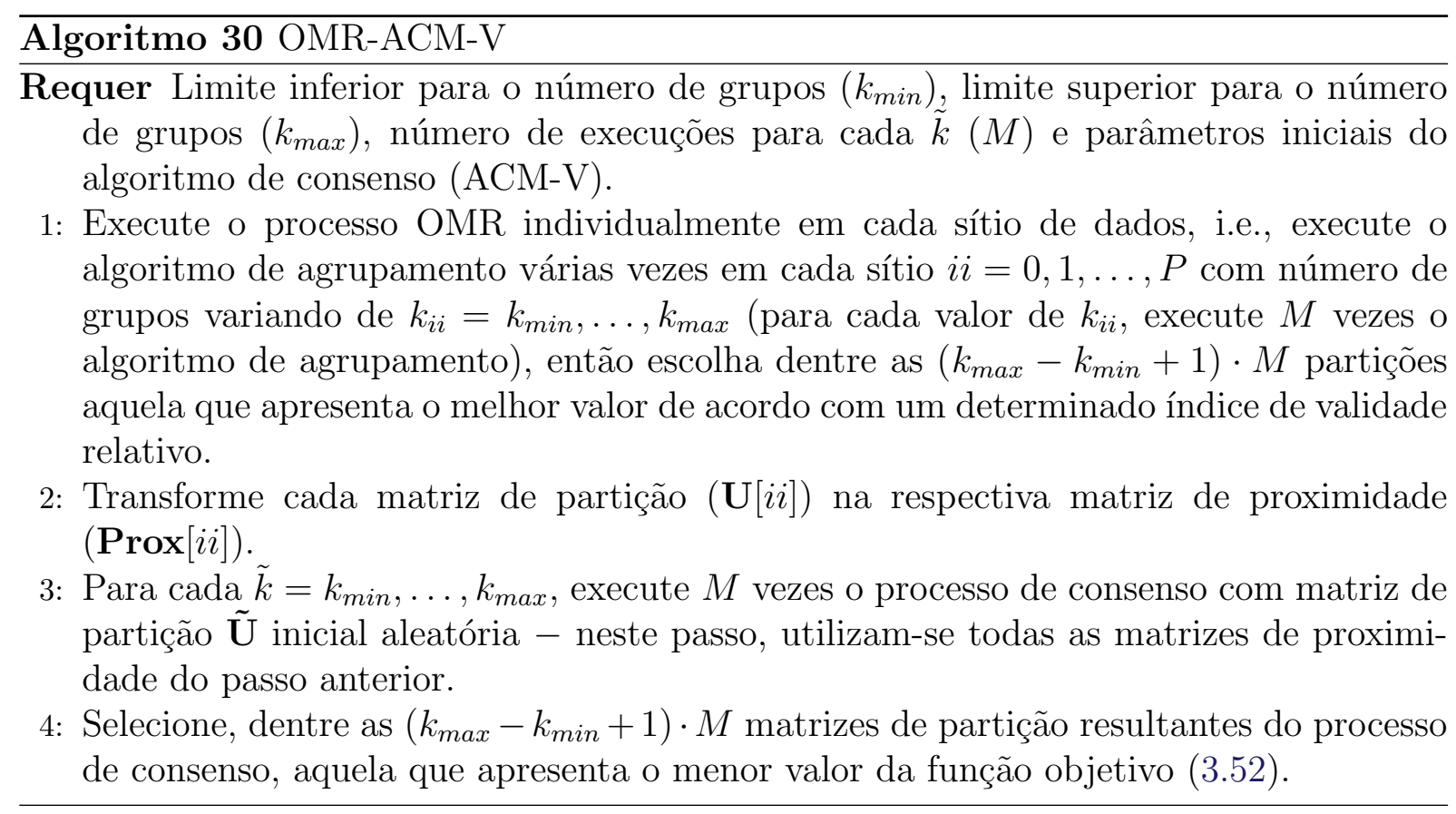




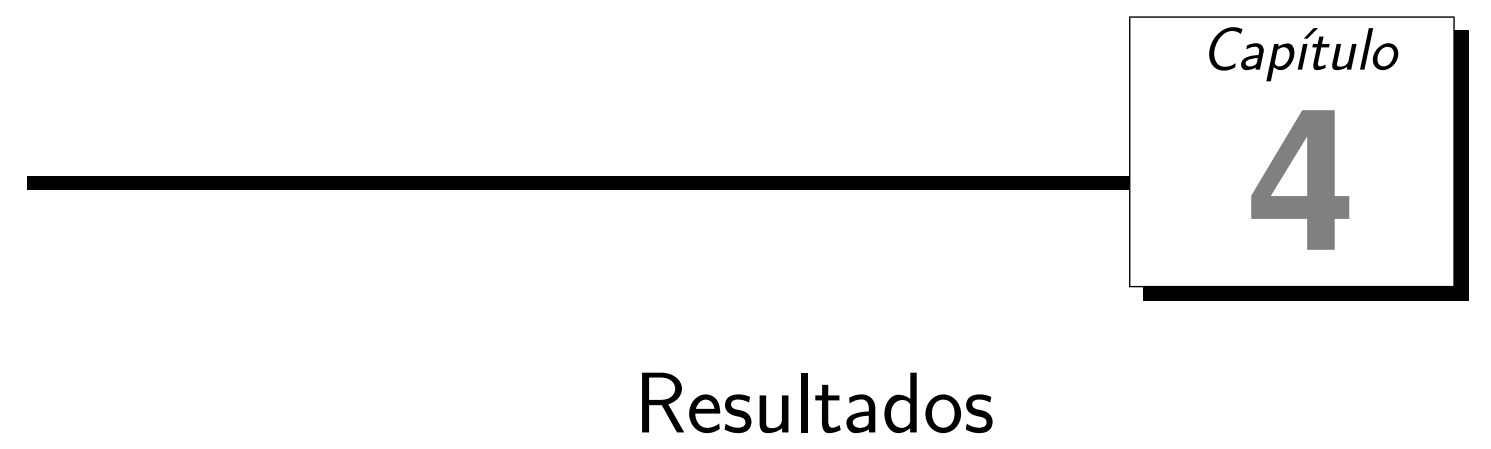

Neste capítulo são apresentados experimentos realizados com os algoritmos generalizados para trabalharem com dados paralelos/distribuídos (Seção 3.1) e com os algoritmos em consenso originais e modificados (Seções 3.2 e 3.3).

\subsection{Experimentos com Algoritmos Paralelos/Distribuídos}

Nesta seção são apresentados experimentos ilustrando o uso de algoritmos de agrupamento generalizados para o cenário paralelo/distribuído usando o framework apresentado na Seção 3.1. O objetivo é ilustrar o speedup ${ }^{1}$ obtido quando o algoritmo de agrupamento é executado em múltiplos processadores em comparação com um único processador ${ }^{2}$.

Os experimentos foram realizados em um computador equipado com um processador Intel 2.8GHz i7-920 de 4 núcleos com 12GB-RAM usando o sistema operacional Linux de 32 bits. Todos os experimentos foram realizados usando Matlab 2009 e a ferramenta Parallel Computing Toolbox do Matlab. Para a execução dos experimentos, três bases de dados foram geradas usando o mesmo gerador descrito em (Milligan \& Cooper, 1981; Vendramin et al., 2010). Cada base de dados contém $k=8$ grupos em um espaço de atributos de $n=8$ dimensões, em que sobreposições não são permitidas na primeira dimensão. A distribuição espacial dos objetos intra-grupo segue uma distribuição normal multivariada truncada, sendo assim a estrutura pode ser considerada conter grupos naturais que exibem as propriedades de separação externa e coesão interna. A primeira base de dados contém $N=2^{10}$ objetos, a segunda base contém $N=2^{13}$ objetos, e a terceira

\footnotetext{
${ }^{1}$ Speedup mede o quão um algoritmo executa mais rapidamente em mais processadores em comparação com um único processador. É definido como a razão entre o tempo de execução de um determinado algoritmo em um único processador pelo tempo de execução do mesmo algoritmo usando mais processadores.

${ }^{2} \mathrm{O}$ objetivo não consiste em comparar as soluções obtidas pelos diferentes algoritmos.
} 
base de dados contém $N=2^{17}$ objetos. A distribuição dos objetos intra-grupos segue uma distribuição normal multivariada (truncada), em que sobreposições não são permitidas na primeira dimensão, de tal forma que a estrutura resultante pode ser considerada de grupos naturais que exibem as propriedades de isolação externa e coesão interna. Os detalhes sobre o gerador de base de dados podem ser encontrados em (Milligan \& Cooper, 1981; Vendramin et al., 2010).

Os algoritmos de agrupamento foram analisados com diferentes números de iterações. Os algoritmos DFCM, DGK, DGG, DPCM, DFPCM e DPFCM foram executados exatamente em 10 e 100 iterações usando um, dois, três e quatro processadores. Em cada caso as bases de dados foram divididas aleatoriamente de forma balanceada entre os processadores. Os parâmetros de execução dos algoritmos foram configurados como $k=8$ (número correto de grupos) e, conforme usual na literatura, $m=\gamma=2, \rho_{i}=1$, $\eta_{i}$ conforme descrito em (2.34) e $a=b=1$.

O speedup obtido pelos algoritmos de agrupamento ao serem executados (10 execuções) nas bases de dados contendo $2^{10}, 2^{13}$ e $2^{17}$ objetos estão ilustradas nas Figuras 4.1, 4.2 e 4.3 , respectivamente.

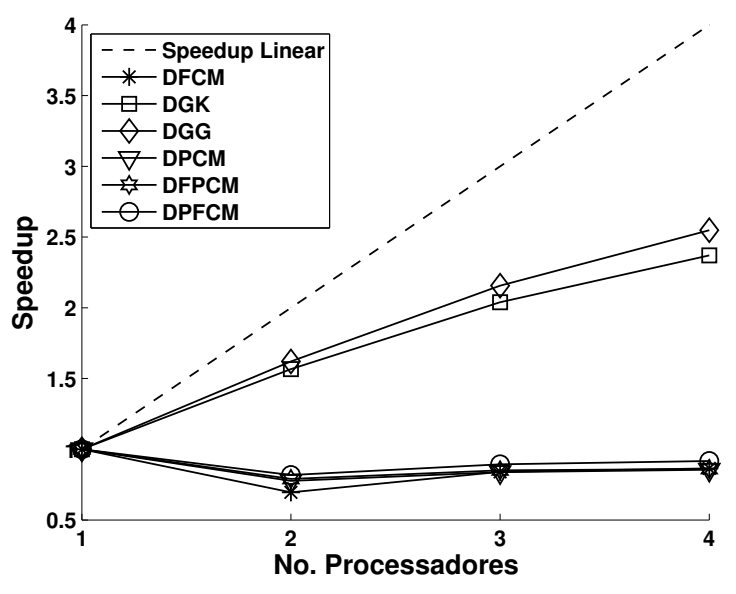

(a) Speedup após 10 iterações.

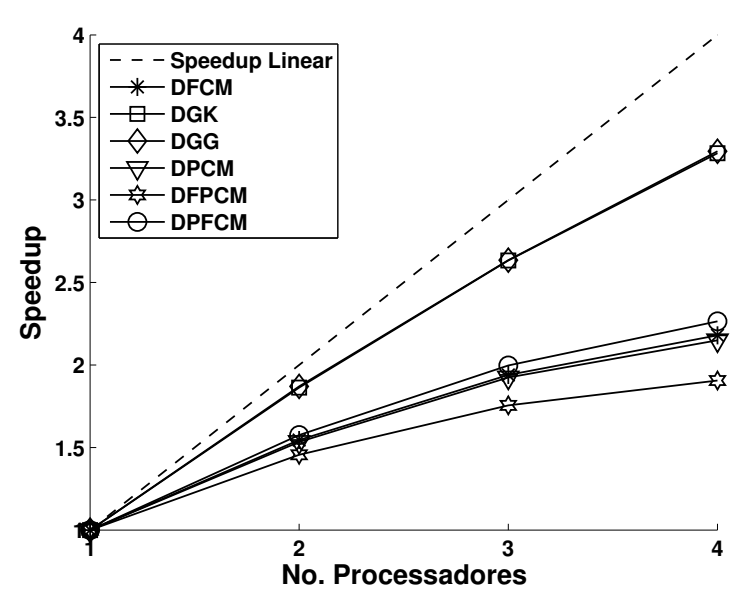

(b) Speedup após 100 iterações.

Figura 4.1: Speedup obtido pelos algoritmos de agrupamento distribuídos executados em uma base de dados com $2^{10}$ objetos após 10 e 100 iterações.

Os resultados ilustrados na Figura 4.1 (base de dados com $2^{10}=1,024$ objetos) mostram que, quando um algoritmo de agrupamento usa mais processadores em uma base de dados pequena, particularmente para um número menor de iterações, seu desempenho pode não melhorar em comparação com sua execução em um único processador, devido a uma alta carga relativa de transferência dos dados (relativa à carga de processamento) e devido à configuração inicial necessária para dividir os dados entre os processadores e iniciar o ambiente paralelo/distribuído ${ }^{3}$. De fato, na Figura 4.1a pode-se notar que os

\footnotetext{
${ }^{3}$ Em bases de dados com poucos objetos o algoritmo de agrupamento executa rapidamente e o tempo necessário para configurar o ambiente paralelo/distribuído pode ser maior do que a própria execução do algoritmo (dependendo do número de iterações). Esse tempo de configuração passa a ter pouca influência
} 


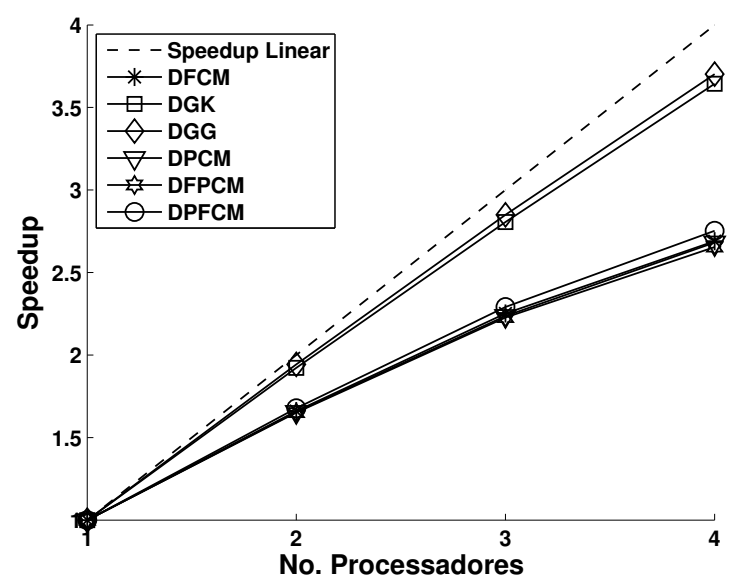

(a) Speedup após 10 iterações.

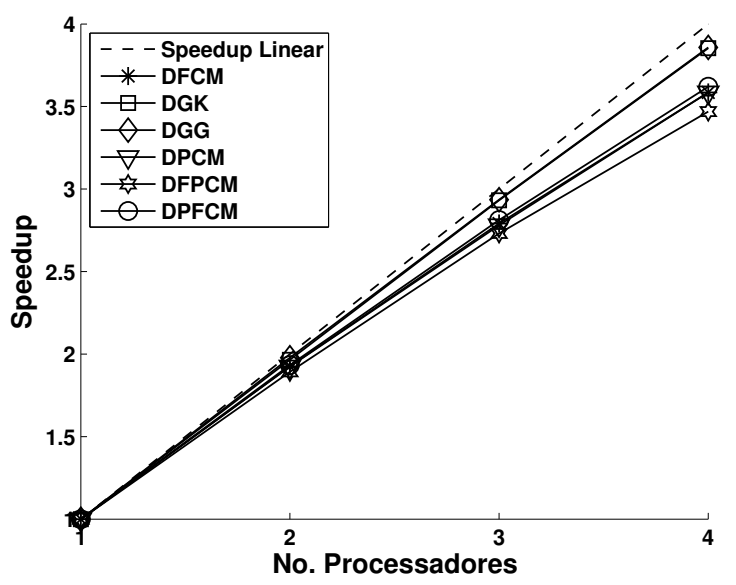

(b) Speedup após 100 iterações.

Figura 4.2: Speedup obtido pelos algoritmos de agrupamento distribuídos executados em uma base de dados com $2^{13}$ objetos após 10 e 100 iterações.

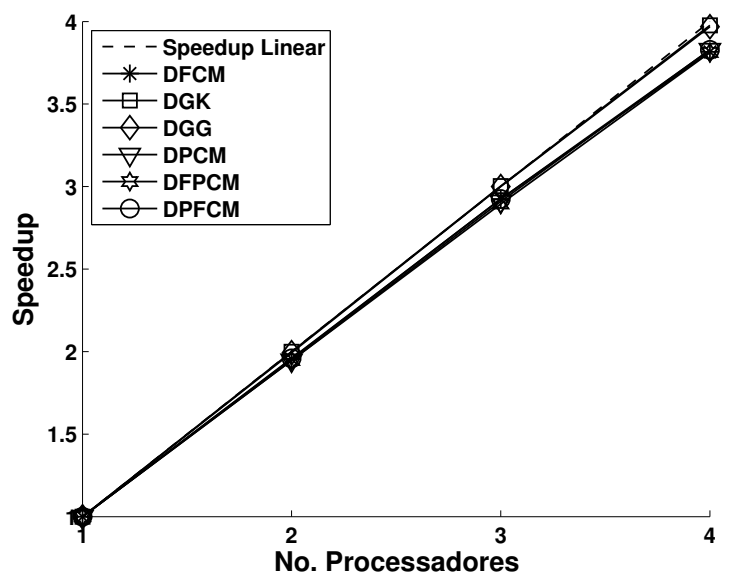

(a) Speedup após 10 iterações.

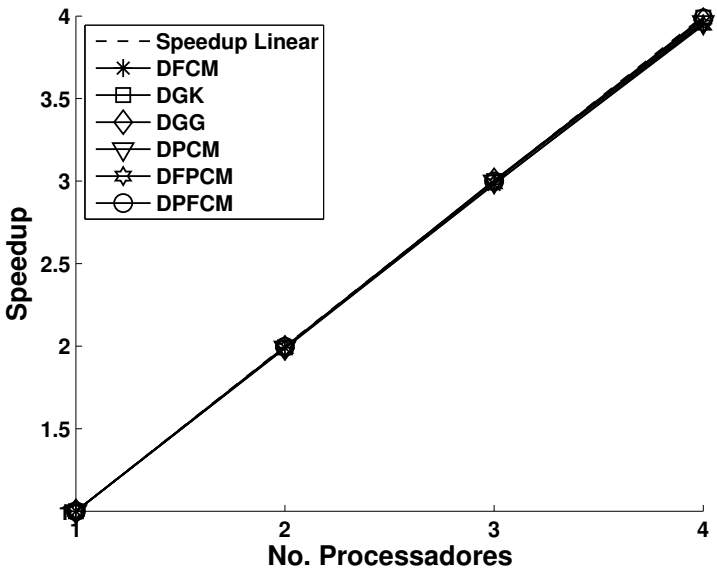

(b) Speedup após 100 iterações.

Figura 4.3: Speedup obtido pelos algoritmos de agrupamento distribuídos executados em uma base de dados com $2^{17}$ objetos após 10 e 100 iterações.

algoritmos de agrupamento, exceto os algoritmos DGK e DGG que calculam matrizes de covariância $^{4}$, levam mais tempo para processar os dados em múltiplos processadores do que levam em um único processador. Contudo, conforme o tamanho da base de dados aumenta, o speedup também aumenta (veja as Figuras 4.2 e 4.3) e, como consequência, o uso de múltiplos processadores se torna uma abordagem interessante para aplicar algoritmos de agrupamento nesses dados.

Na Tabela 4.1 são descritas as quantidades de transferência (em Kbytes) entre os sítios de dados durante a execução dos algoritmos após 100 iterações. As quantidades de transferências são proporcionais ao número de iterações dos algoritmos. Assim, foi

quando as bases de dados apresentam muitos objetos e, consequentemente, o algoritmo de agrupamento permanece muito tempo em execução.

${ }^{4} \mathrm{O}$ cálculo das matrizes de covariância e suas inversas é um processamento custoso, o que faz com que tais algoritmos possuam maior razão entre tempo de processamento e carga de transmissão. 
observado um décimo das quantidades da Tabela 4.1 após 10 iterações dos algoritmos. Note que a quantidade de transferência não depende do tamanho da base de dados, uma vez que os algoritmos transferem apenas informações sumarizadas (como protótipos, somas das pertinências, entre outros). Dessa forma, a mesma quantidade de transferência foi observada para as bases de dados formadas por $N=2^{10}, N=2^{13}$ e $N=2^{17}$ objetos. Isso explica por que o speedup aumenta com o tamanho da base de dados, pois a razão entre tempo de processamento e carga de transmissão também aumenta.

\begin{tabular}{lc|c|c}
\hline Processadores & Dois & Três & Quatro \\
\hline DFCM & 1.125 & 1.750 & 2.375 \\
DGK & 9.250 & 14.000 & 18.750 \\
DGG & 9.500 & 14.438 & 19.375 \\
DPCM & 1.125 & 1.750 & 2.375 \\
DFPCM & 1.375 & 2.188 & 3.000 \\
DPFCM & 1.125 & 1.750 & 2.375 \\
\hline
\end{tabular}

Tabela 4.1: Quantidade de transferência (em Kbytes) entre os sítios de dados dos algoritmos usando dois, três e quatro processadores após 100 iterações.

\subsection{Experimentos com Algoritmos de Consenso}

Antes de prosseguir com os experimentos relacionados ao agrupamento em consenso, é importante deixar claro que o algoritmo FCM e suas variantes foram executados com coeficiente de fuzzyficação $m=2$ e critério de parada $\epsilon=10^{-3}$ que são valores usualmente adotados na literatura (Bezdek, 1981; Babuska, 1998; Hoppner et al., 1999). Os índices de validade XB e DXB foram usados para avaliar a qualidade das partições nos procedimentos OMR-FCM e OMR-DFCM, respectivamente ${ }^{5}$. Finalmente, os pesos de cada sítio de dados usados pelo processo de consenso foram $\alpha_{i i}=1, \forall i i$.

O objetivo deste experimento consiste em demonstrar as limitações existentes no processo de consenso original (Seções 3.2.1 e 3.3.1) em comparação com o aperfeiçoamento proposto neste trabalho (Seções 3.2.2 e 3.3.2). Uma base de dados, ilustrada na Figura 4.4a, com $N=30$ objetos descritos por $n=2$ atributos, é utilizada para a realização deste experimento.

\footnotetext{
${ }^{5}$ Resultados similares foram obtidos com o índice de validade FSS e DFSS.
} 


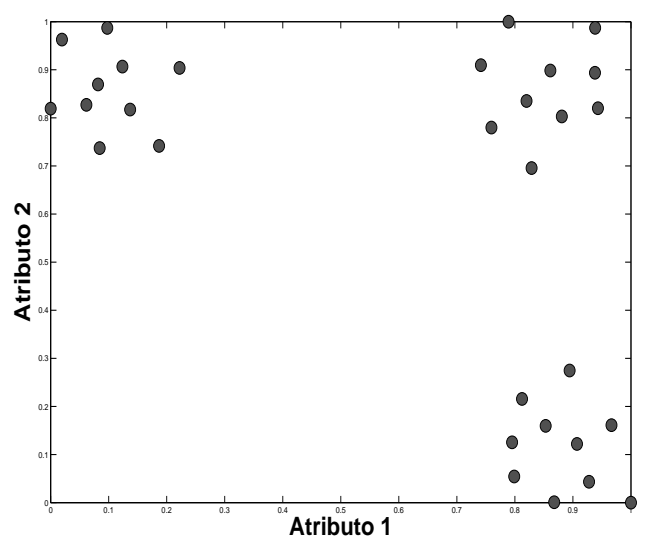

(a) Base de dados com $N=30$ objetos descritos por (b) Resultado obtido pelo algoritmo FCM $\operatorname{com} k=3$ $n=2$ atributos.

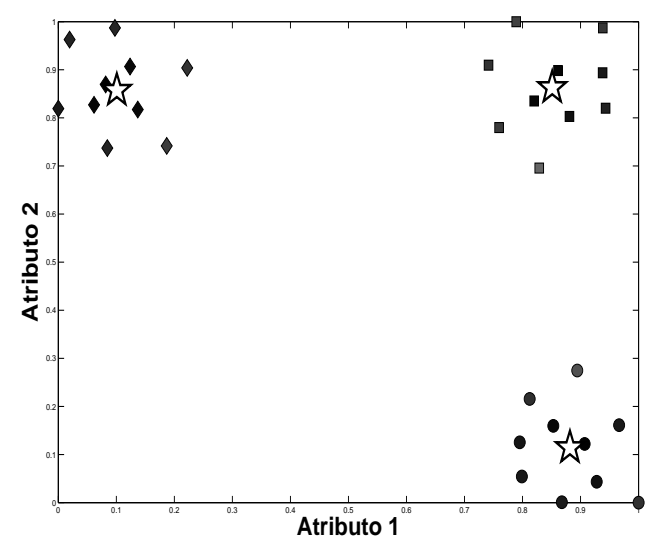

grupos (círculos, quadrados e diamantes) - centros são representados por "estrelas" — o maior valor de pertinência de cada objeto é representado pela intensidade de preto.

Figura 4.4: Base de dados de exemplo e o resultado do algoritmo FCM.

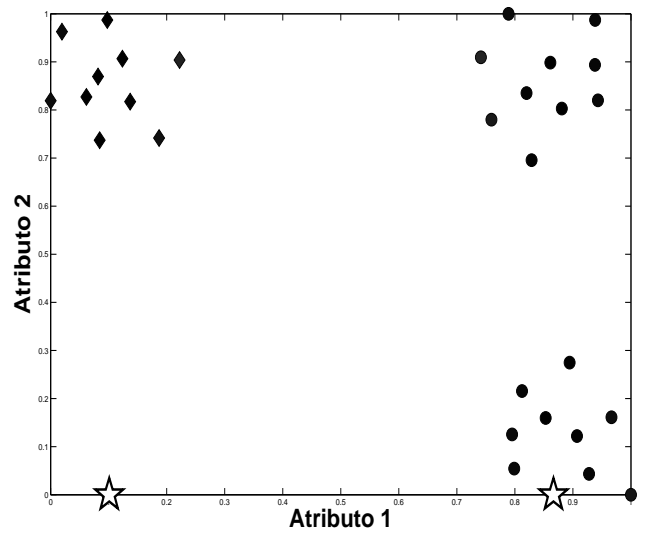

(a) Resultado do agrupamento no primeiro subespaço (atributo).

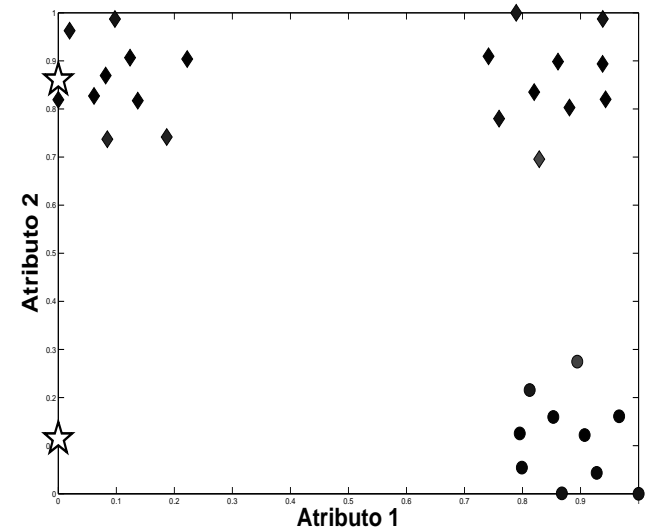

(b) Resultado do agrupamento no segundo subespaço (atributo).

Figura 4.5: Resultado do algoritmo FCM aplicado individualmente em cada subespaço com $k=2$ grupos — centros são representados por "estrelas" — o maior valor de pertinência de cada objeto é representado pela intensidade de preto.

O resultado obtido pelo algoritmo de agrupamento ao ser executado nessa base de dados, com $k=3$ grupos, está ilustrado na Figura 4.4b. Considere, entretanto, que os dados são descritos por diferentes subespaços (cada atributo descreve um subespaço). Ao aplicar o algoritmo de agrupamento FCM, com $k=2$ grupos, no primeiro subespaço (primeiro atributo), obtém-se o resultado ilustrado na Figura 4.5a. Similarmente, ao aplicar o mesmo algoritmo de agrupamento FCM, com $k=2$, no segundo subespaço, obtém-se o resultado ilustrado na Figura 4.5b.

Um algoritmo de consenso pode utilizar os resultados obtidos individualmente em cada subespaço (Figura 4.5) para encontrar uma estrutura que reconcilie ambos resultados ob- 


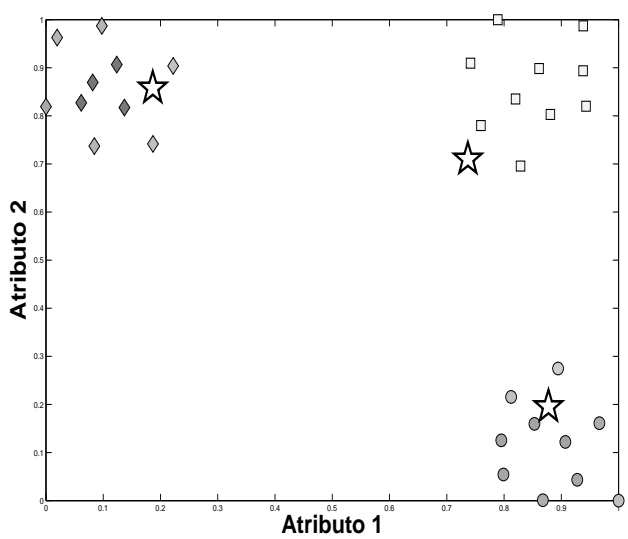

(a) Resultado do algoritmo ACO-V executado com matriz partição inicial referente à solução ilustrada na Figura 4.4b.

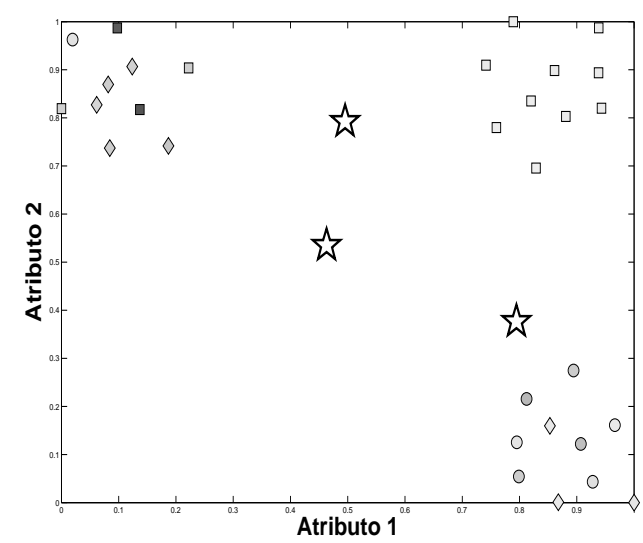

(b) Resultado do algoritmo ACO-V executado com matriz partição inicial aleatória.

Figura 4.6: Resultado do algoritmo ACO-V executado para as soluções obtidas individualmente em cada subespaço ilustradas na Figura 4.5 - centros são representados por "estrelas" - o maior valor de pertinência de cada objeto é representado pela intensidade de preto.

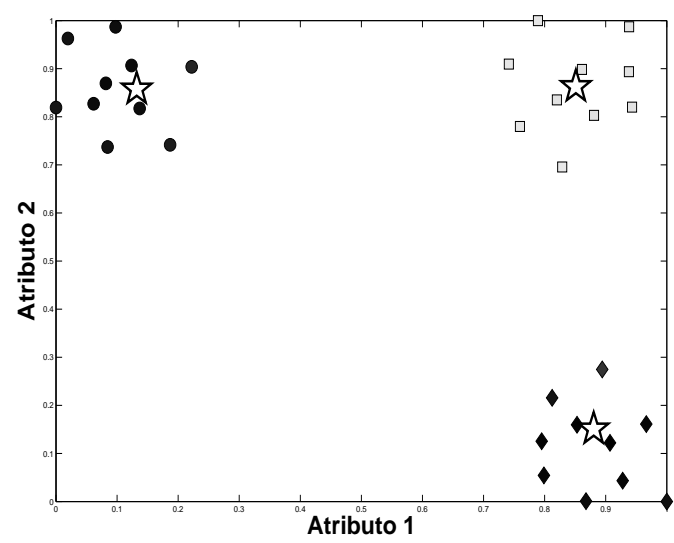

Figura 4.7: Resultado do algoritmo ACM-V executado para as soluções obtidas individualmente em cada subespaço ilustradas na Figura 4.5 - centros são representados por "estrelas" - o maior valor de pertinência de cada objeto é representado pela intensidade de preto.

tidos. Se o algoritmo ACO-V (algoritmo de consenso original) (Seção 3.3.1) for executado, com $\tilde{k}=3$ e matriz de partição inicial dada pela solução ilustrada na Figura 4.4b (que seria obtida se os dados estivessem centralizados), obtém-se o resultado ilustrado na Figura 4.6a. Similarmente, se o algoritmo ACO-V for executado, com $\tilde{k}=3$ e matriz de partição inicial aleatória, obtém-se o resultado ilustrado na Figura 4.6b.

Claramente, o algoritmo de consenso original apresenta dificuldades em encontrar uma solução consenso. Mesmo com uma matriz de partição inicial próxima da esperada (Figura 4.4b), o processo apresenta dificuldades em encontrar valores de pertinências condizentes com a estrutura de grupos naturais. Note que a pertinência de cada objeto aos grupos 
(representada na Figura 4.4b pela intensidade de preto de cada objeto) foi enfraquecida (i.e., um objeto pertence a mais de um grupo com grau similar) e, consequentemente, os centros dos grupos estão em locais inesperados. Se, entretanto, a versão modificada do algoritmo de consenso (ACM-V) (Seção 3.3.2) for executada, com $\tilde{k}=3$ e matriz de partição inicial aleatória (resultados similares são encontrados ao utilizar a matriz de partição inicial referente à Figura 4.4b), obtém-se o resultado ilustrado na Figura 4.7.

Claramente o processo de otimização do algoritmo ACM-V consegue convergir para uma solução consenso mais adequada do que a solução obtida pelo processo original. Note que, na Figura 4.7, os protótipos dos grupos estão no centro dos três grupos visualmente esperados.

\subsection{Caso Horizontal - I}

Este experimento conta com dois sítios de dados, cada qual com $N=40$ objetos distintos descritos pelos mesmos $n=2$ atributos. Os sítios de dados estão ilustrados na Figura 4.8. O objetivo do usuário no domínio de um sítio de dados é melhorar os resultados obtidos localmente a partir de informações provenientes do outro sítio de dados.

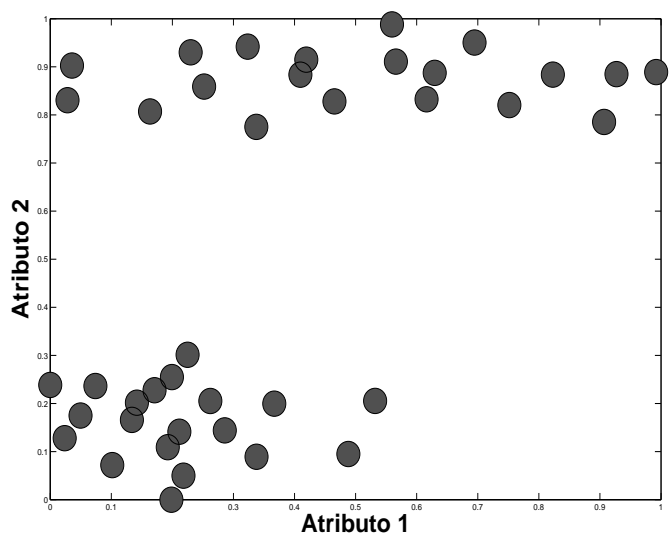

(a) Sítio de Dados 1.

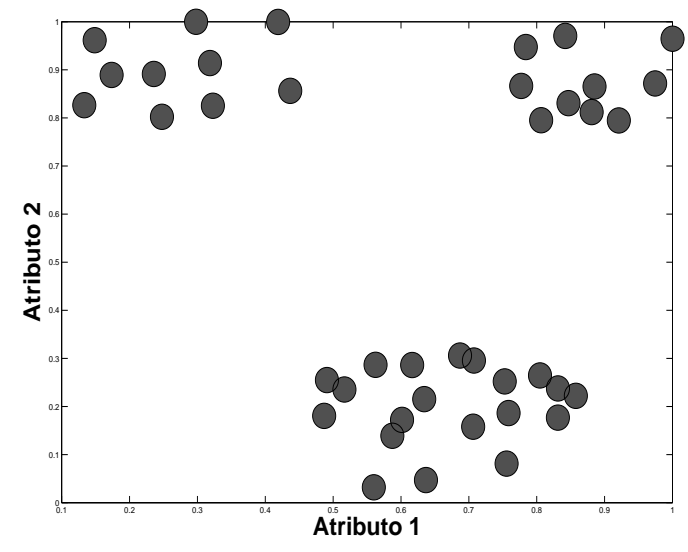

(b) Sítio de Dados 2.

Figura 4.8: Dois sítios de dados. Cada sítio de dados possui $N=40$ objetos descritos pelos mesmos $n=2$ atributos.

Uma das abordagens para agrupar dados distribuídos consiste em rodar um algoritmo de agrupamento distribuído que produza o mesmo resultado que seria obtido enviando os dados para um único local (centralizado) e aplicando um algoritmo de agrupamento centralizado. O procedimento DOMR-DFCM (Seção 3.1.6), com $k_{\min }=2, k_{\max }=10 \mathrm{e}$ $M=20$, foi utilizado para essa abordagem. O resultado desse procedimento, com quatro grupos (círculos, quadrados, diamantes e triângulos), está ilustrado na Figura 4.9. O mesmo resultado é ilustrado na Figura 4.10 para os sítios de dados separadamente.

Note que os resultados da Figura 4.9 ilustram ambos sítios de dados juntos (como se os dados fossem centralizados em um único local). Visualmente, o resultado (com 


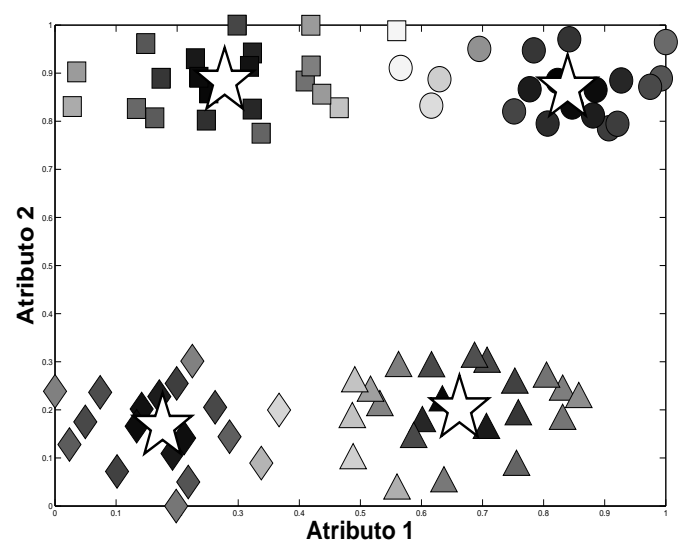

Figura 4.9: Melhor partição fuzzy obtida pelo procedimento DOMR-DFCM para os sítios de dados ilustrados na Figura 4.8. Os sítios de dados estão ilustrados juntos (centralizados) — os centros são representados por "estrelas" — o maior valor de pertinência de cada objeto é representado pela intensidade de preto.

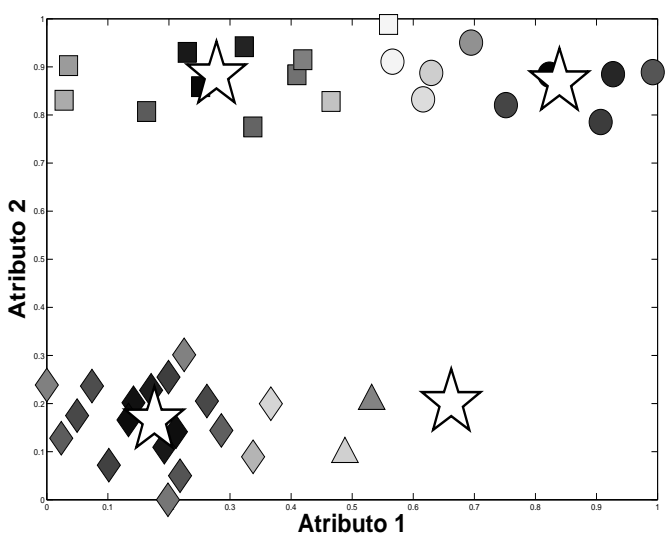

(a) Sítio de Dados 1 .

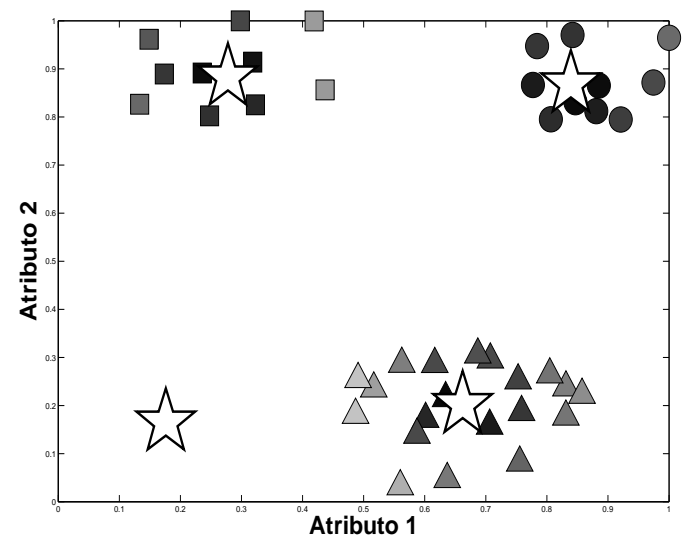

(b) Sítio de Dados 2.

Figura 4.10: Melhor partição fuzzy obtida pelo procedimento DOMR-DFCM para os sítios de dados da Figura 4.8. Cada sítio de dados é ilustrado separadamente - os centros são representados por "estrelas" — o maior valor de pertinência de cada objeto é representado pela intensidade de preto.

$k=4$ grupos) encontrado pelo procedimento DOMR-DFCM reflete a estrutura de grupos esperada. Entretanto, uma análise individual dos sítios de dados na Figura 4.10 mostra que os $k=4$ grupos dificilmente refletem a estrutura local. Embora esse resultado global possa ser útil para os sítios de dados, notificando-os da existência de mais grupos que o individualmente identificado, ele não considera que cada sítio pode ter características próprias, à parte das características em comum com os demais sítios. Note que os objetos dispostos na parte inferior da Figura 4.10a foram divididos em dois grupos (diamantes e triângulos). Isso se deve ao fato de existirem objetos no mesmo local no outro sítio de dados (Figura 4.10b) (triângulos). Essa divisão pode ser prejudicial para o sítio de dados 1, uma vez que não existem objetos onde o novo grupo foi identificado. De fato, pode ser 


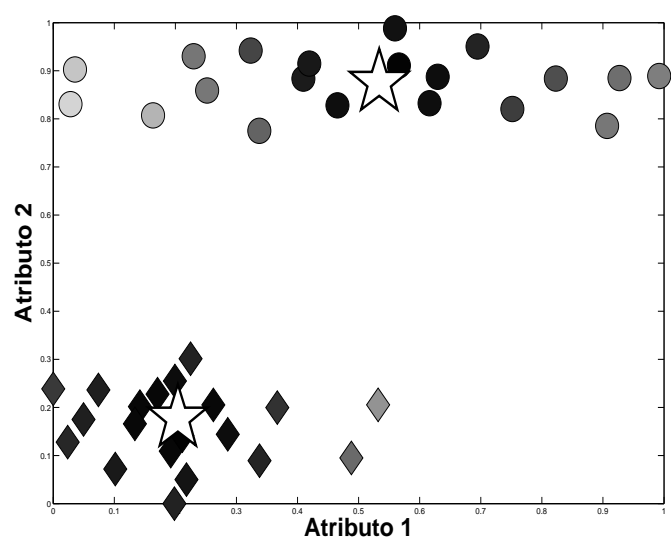

(a) Sítio de Dados 1 .

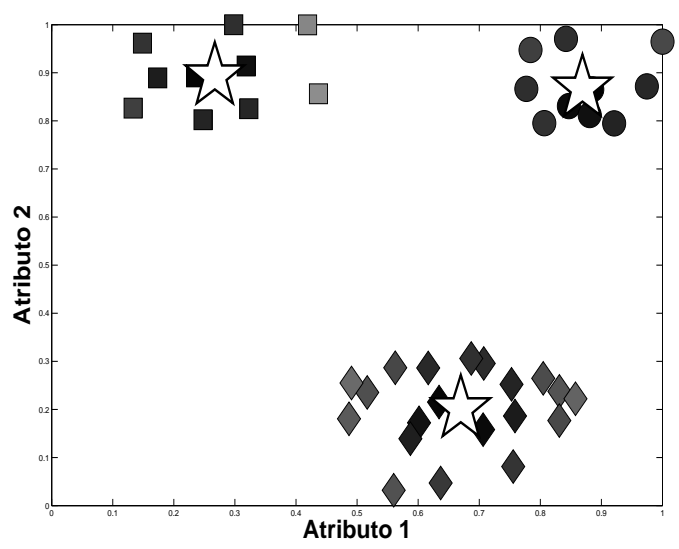

(b) Sítio de Dados 2 .

Figura 4.11: Melhor partição obtida pelo procedimento DOMR-FCM quando aplicado a cada sítio de dados individualmente.

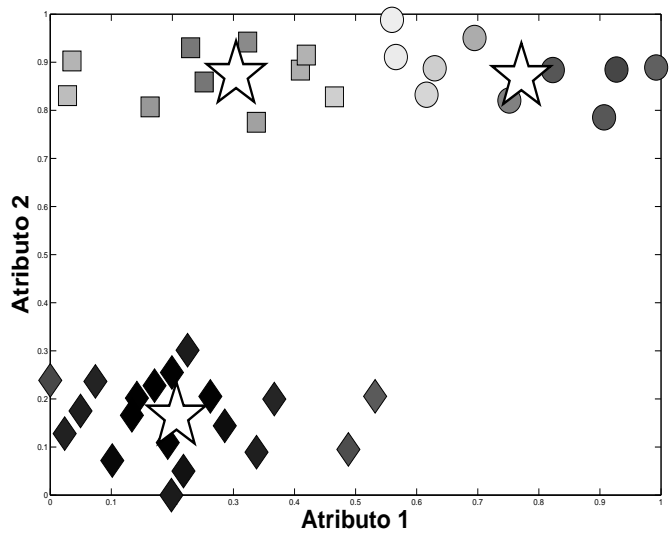

(a) Sítio de Dados 1 .

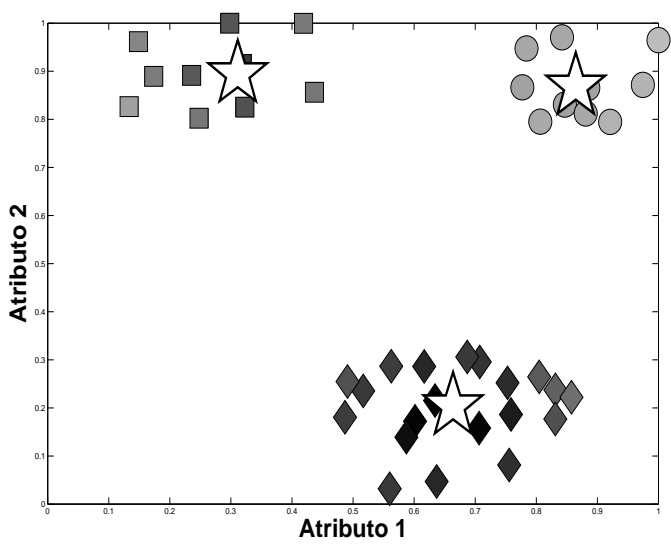

(b) Sítio de Dados 2 .

Figura 4.12: Melhor resultado obtido pelo processo de consenso: (a) consenso aplicado considerando o sítio de dados 1 como local; (b) consenso aplicado considerando o sítio de dados 2 como local.

preferível um único grupo ao invés de dividi-lo em dois grupos naquela região — dado que um desses grupos terá somente dois objetos e as pertinências dos outros objetos serão enfraquecidas. O sítio de dados 2, por sua vez, possui um grupo não representativo.

Nesses casos é desejável o uso de um algoritmo de agrupamento que melhore o resultado obtido em cada sítio de dados (com informações fornecidas por outros sítios) sem prejudicar o resultado local existente. Em outras palavras, é esperado que o resultado obtido localmente em cada sítio de dados não seja drasticamente alterado, exceto por mudanças que reflitam forte evidência presente em vários sítios de dados simultaneamente. Para essa abordagem, o procedimento OMR-ACM-H (Seção 3.2.3) foi adotado. Primeiramente, o procedimento OMR-FCM foi executado em cada sítio de dados individualmente com número de grupos variando de $k_{i i}=2$ até $k_{i i}=10$ e $M=20$ execuções do algoritmo FCM para cada valor de $k_{i i}$. As soluções encontradas pelo algoritmo OMR-FCM aplicado 
individualmente em cada sítio de dados estão ilustradas na Figura 4.11. Essas partições (com $k_{1}=2$ e $k_{2}=3$ grupos) foram usadas no processo de consenso. O procedimento OMR-ACM-H foi então aplicado a cada sítio de dados (considerando-o como sítio de dados local, $\mathbf{X}[0]$ ) com o mesmo intervalo de número de grupos. Para cada número de grupos, o número máximo de iterações do processo de otimização foi 200. A solução que resultou no menor valor de função objetivo, quando os sítios de dados 1 e 2 foram considerados como sítio de dados local, estão ilustrados respectivamente nas Figuras 4.12a e 4.12b. Note que o processo de consenso guiou o sítio de dados 1 a identificar a existência de três grupos. Os objetos que estão ilustrados na parte superior da Figura 4.12a foram divididos em dois grupos (quadrados e círculos). Isso se deve à forte evidência de dois grupos nessa região identificados pelo sítio de dados 2 (Figura 4.11b) e que estão menos evidentes no sítio de dados 1. Contudo, o processo de consenso encontrou somente um grupo na parte inferior das Figuras 4.12a e 4.12b (diamantes), que pode ser mais desejado, dado que não existe evidência de outro grupo ao analisar cada sítio de dados individualmente.

Este experimento ilustra um cenário em que o processo de consenso pode ser benéfico ao sítio de dados local. Com a informação disponibilizada pelo outro sítio de dados, o sítio de dados 1 identificou a existência de dois grupos (ao invés de um único grupo) na parte superior da Figura 4.12a. Entretanto, o grupo ilustrado na parte inferior da Figura 4.12a não foi dividido em dois grupos como ocorreu com o procedimento OMR-DFCM ilustrado na Figura 4.10a. Tal comportamento pode ser mais desejável para o sítio de dados local, dado que não existem evidências da existência desses grupos ao analisar o sítio de dados 1 individualmente.

\subsection{Caso Horizontal - /I}

Este experimento conta com três sítios de dados, cada qual com $N=50$ objetos distintos descritos pelos mesmos $n=2$ atributos. Os sítios de dados estão ilustrados na Figura 4.13. Novamente, o objetivo do usuário no domínio de um sítio de dados é melhorar os resultados locais obtidos com informações provenientes do outro sítio de dados.

Conforme discutido anteriormente, uma das abordagens para agrupar dados distribuídos consiste em rodar um simples algoritmo de agrupamento distribuído. Neste experimento, o procedimento OMR-DFCM foi novamente utilizado para essa abordagem, com

$k_{\min }=2, k_{\max }=10$ e $M=20$. O resultado desse procedimento, com dois grupos (círculos e diamantes), está ilustrado na Figura 4.14.

Note que os resultados da Figura 4.14 ilustram ambos sítios de dados juntos (como se os dados fossem centralizados em um único local). Visualmente, o resultado (com $k=2$ grupos) encontrado pelo procedimento OMR-DFCM não reflete a estrutura de grupos esperada porque, nesse caso em particular, o índice de validade relativo distribuído utilizado (Seção 3.1.5.4) falhou em detectar que o grupo na parte inferior da figura é na verdade composto de 3 subgrupos razoavelmente bem definidos. Os resultados obtidos pelo proce- 


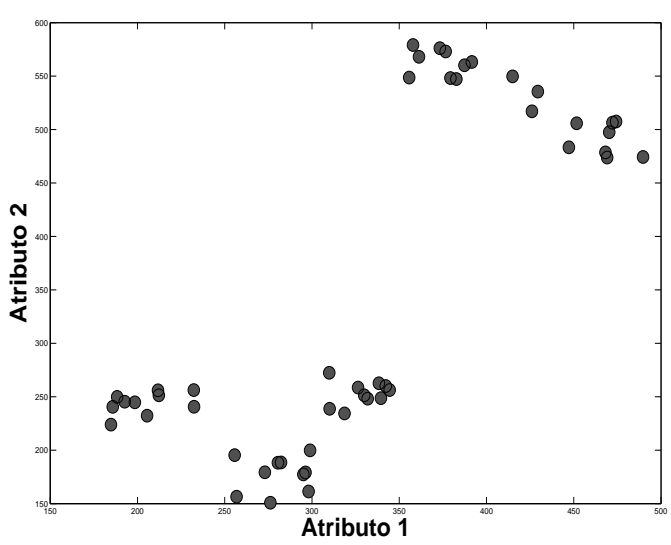

(a) Sítio de Dados 1 .

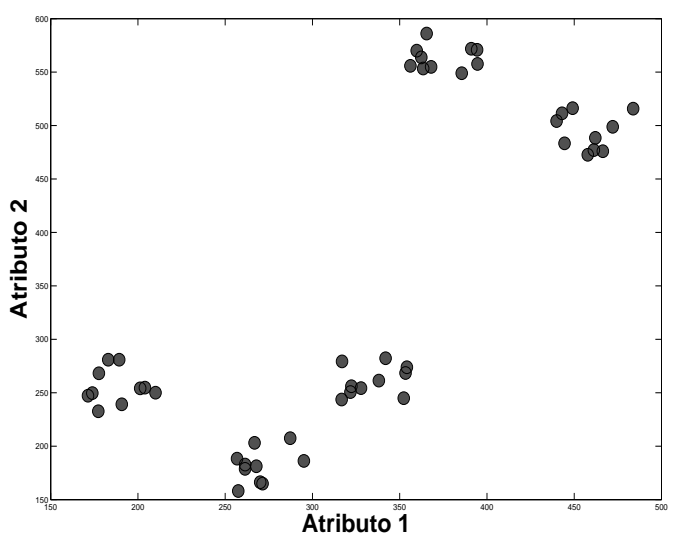

(b) Sítio de Dados 2 .

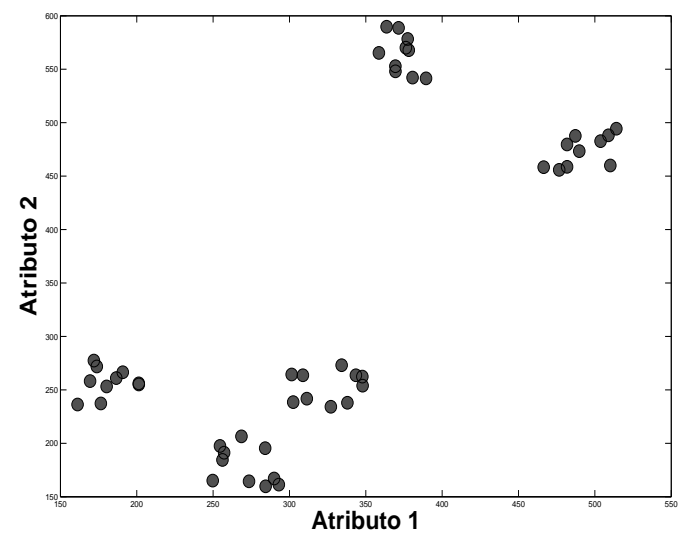

(c) Sítio de Dados 3 .

Figura 4.13: Três sítios de dados. Cada um possui $N=50$ objetos descritos por $n=2$ atributos.

dimento OMR-FCM aplicado individualmente em cada sítio de dados estão ilustrados na Figura 4.15. Note que o sítio de dados 1 encontrou $k_{1}=2$ grupos (círculos e diamantes) cujos centros são similares aos encontrados pelo procedimento OMR-DFCM. Os outros sítios de dados encontraram $k_{2}=k_{3}=5$ grupos (círculos, diamantes, quadrados, triângulos e triângulos invertidos), representando uma estrutura que pode ser mais aceitável visualmente.

O processo de consenso pode ajudar o sítio de dados 1 a encontrar mais grupos do que os $k_{1}=2$ grupos encontrados individualmente. A evidência da existência de $k_{2}=k_{3}=5$ grupos em outros sítios de dados pode auxiliar a encontrar uma estrutura que é mais representativa no sítio de dados 1. Para esse fim, o procedimento OMR-ACM-H foi aplicado com os resultados dos sítios de dados ilustrados na Figura 4.15. O resultado desse procedimento está ilustrado na Figura 4.16. Note que o processo de consenso guiou o sítio de dados 1 a identificar a existência de quatro grupos. Os objetos da parte inferior da Figura 4.15a foram divididos em três grupos (círculos, triângulos e quadrados). Isso se deve à evidência de três grupos nesta região identificada pelos sítios de dados 2 e 3 


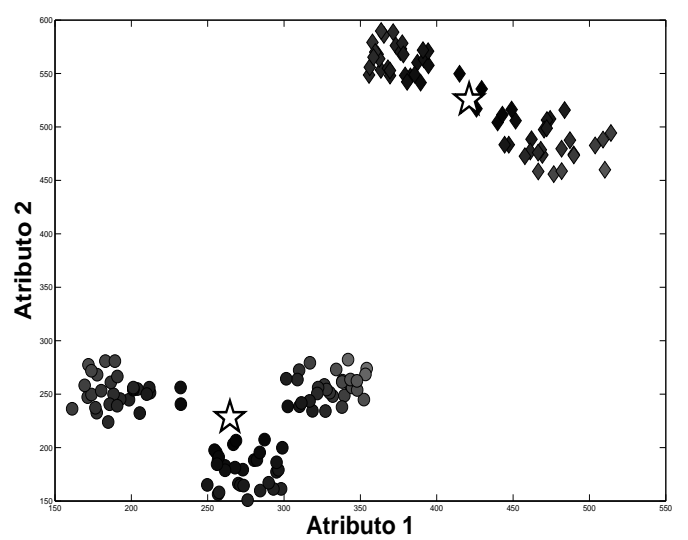

Figura 4.14: Melhor partição fuzzy obtida pelo procedimento OMR-DFCM para os sítios de dados da Figura 4.13.

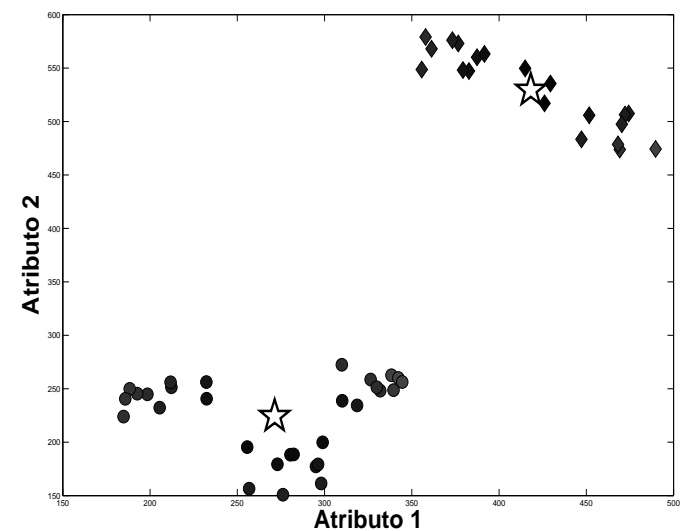

(a) Sítio de Dados 1

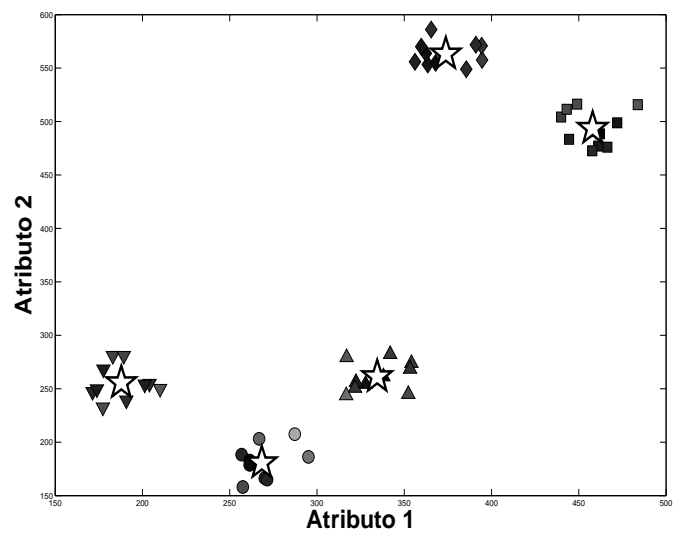

(b) Sítio de Dados 2

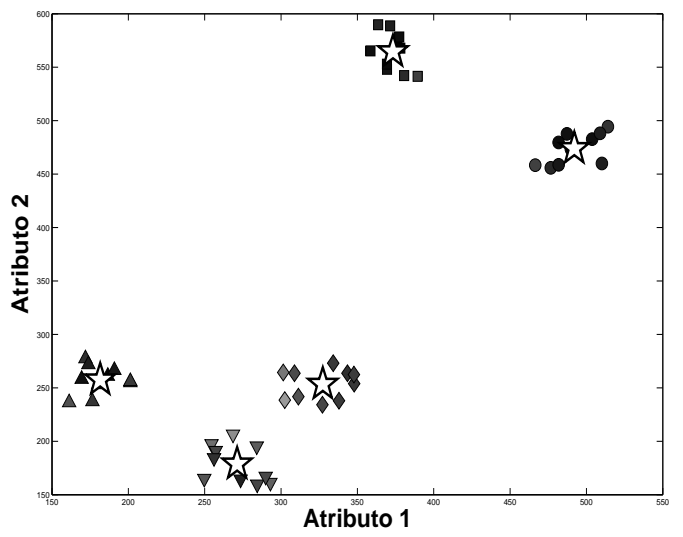

(c) Sítio de Dados 3

Figura 4.15: Melhor partição obtida pelo procedimento OMR-FCM em cada sítio de dados individualmente.

(Figuras $4.15 \mathrm{~b}$ e $4.15 \mathrm{c}$ ) e que também estão presentes no sítio 1. Note, entretanto, que a evidência de dois grupos na parte superior da Figura 4.15a não foi suficiente para o sítio de dados 1. Apesar disso, este resultado ilustra um cenário em que o processo de 


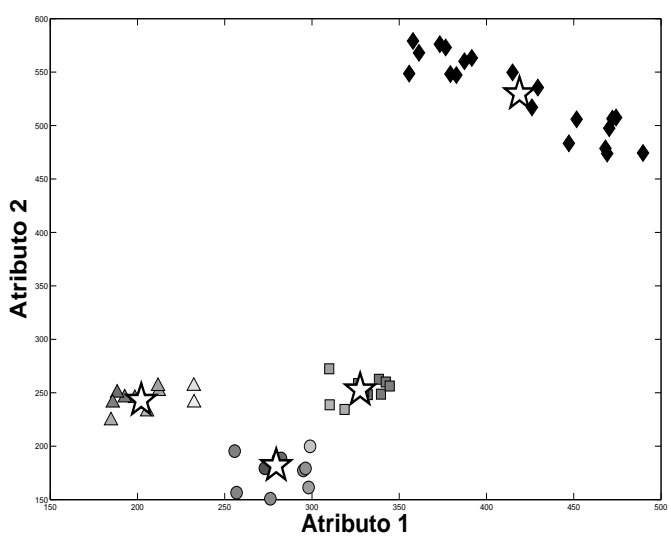

(a) Sítio de Dados 1

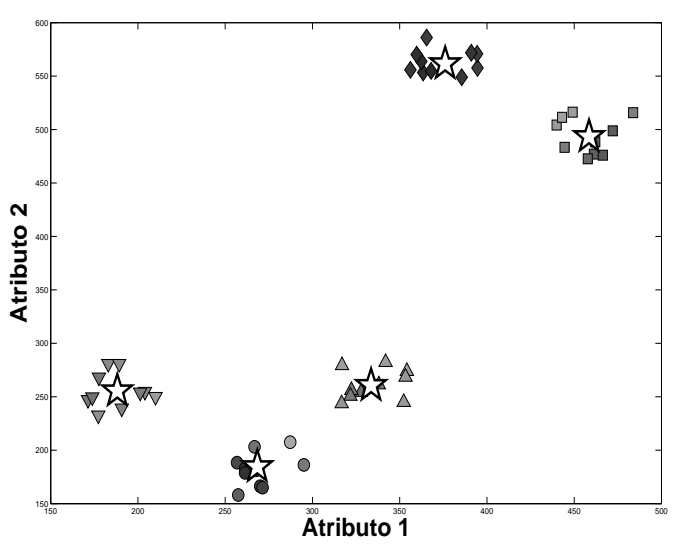

(b) Sítio de Dados 1

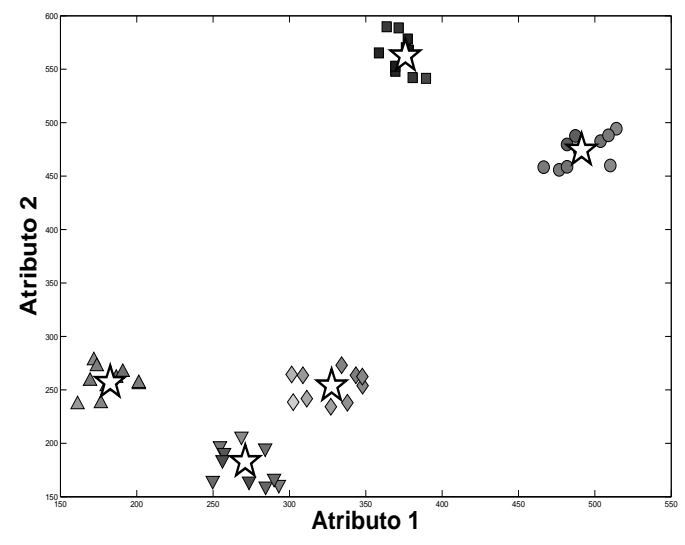

(c) Sítio de Dados 1

Figura 4.16: Resultado obtido pelo processo de consenso: (a) consenso aplicado considerando o sítio de dados 1 como local; (b) consenso aplicado considerando o sítio de dados 2 como local; (c) consenso aplicado considerando o sítio de dados 3 como local.

consenso pode ser benéfico ao sítio de dados local. As informações dos outros sítios de dados ajudaram a identificar a existência de quatro grupos mais aceitáveis (ao invés de dois grupos) no sítio de dados 1 . 


\subsection{Caso Vertical - I}

Este experimento conta com uma base de dados contendo quatro grupos naturais descritos por $n=2$ atributos, no qual cada atributo pode ser interpretado como um subespaço (sítio de dados), conforme ilustrado na Figura 4.17.

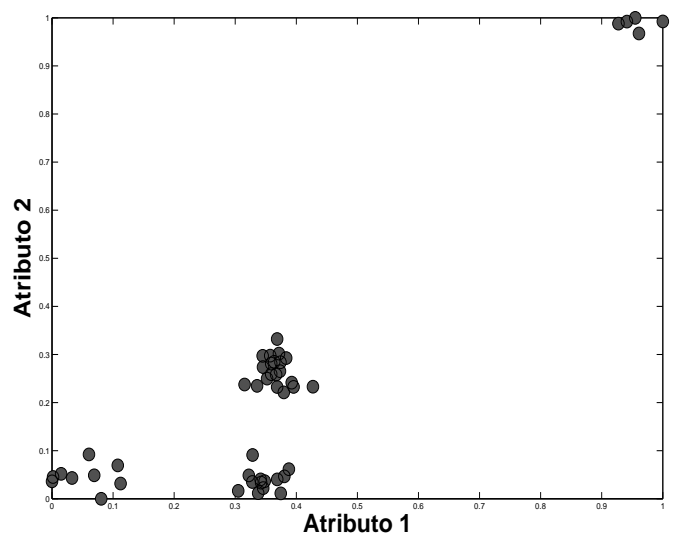

Figura 4.17: Base de dados com quatro grupos naturais descritos por $n=2$ atributos.

O procedimento OMR-FCM foi aplicado à base de dados original (com os dois atributos) com $k_{\text {min }}=2, k_{\text {max }}=10$ e $M=20$. A solução obtida por esse procedimento está ilustrada na Figura 4.18. Note que se obteve uma partição com somente dois grupos (círculos e diamantes). O procedimento OMR-FCM também foi aplicado em cada dimensão separadamente, para os mesmos valores de $k_{\text {min }}, k_{\max }$ e $M$. Os resultados (com três grupos - círculos, diamantes e quadrados) obtidos separadamente em cada subespaço estão ilustrados nas Figuras 4.19 e 4.20 para os subespaços 1 e 2, respectivamente. Note que, devido às diferentes características dos objetos em cada subespaço, o resultado do processo de agrupamento é significativamente diferente nos diferentes subespaços. Somente um dos grupos apresentam os mesmos objetos em ambos subespaços. Entretanto, é possível identificar uma relação entre os resultados obtidos em cada subespaço, reforçando a idéia de que um processo de consenso pode ser benéfico. 


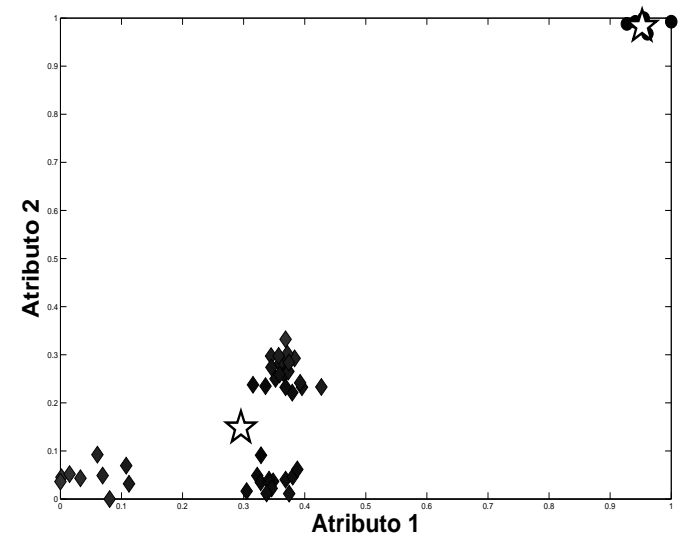

Figura 4.18: Solução encontrada pelo procedimento OMR-FCM na base de dados da Figura 4.17

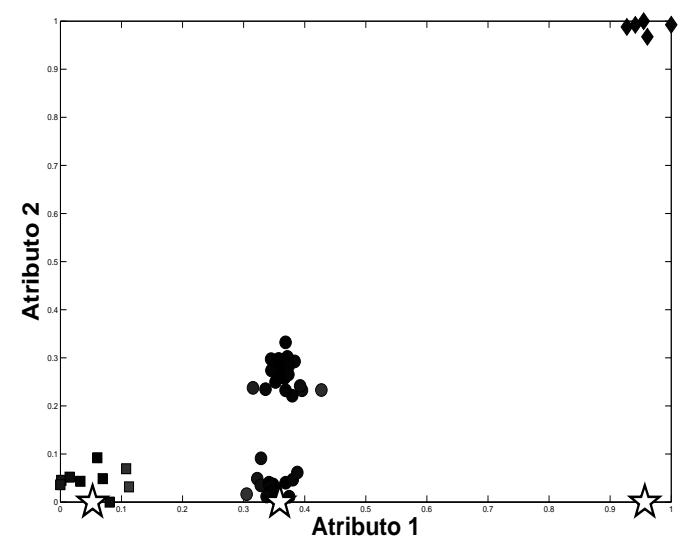

Figura 4.19: Solução encontrada pelo procedimento OMR-FCM no primeiro subespaço.

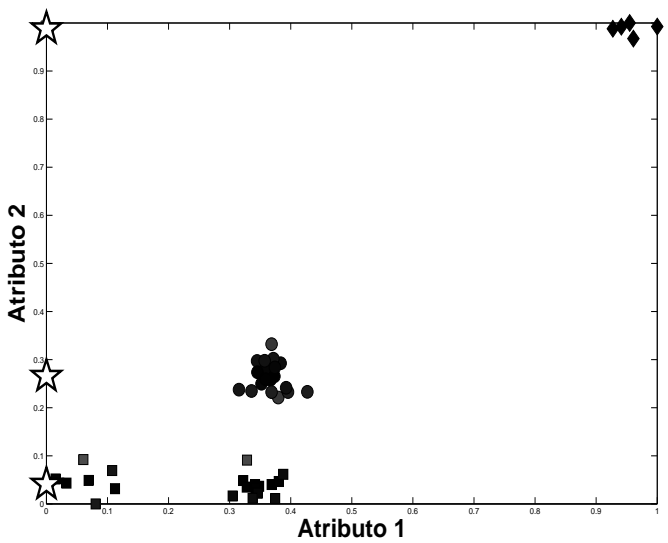

Figura 4.20: Solução encontrada pelo procedimento OMR-FCM no segundo subespaço.

As soluções encontradas pelo processo OMR-FCM em cada subespaço, ilustradas nas Figuras 4.19 e 4.20, foram usadas no processo de consenso. O procedimento OMR-ACM-V 
foi aplicado com o mesmo intervalo de número de grupos. O número máximo de iterações do processo de otimização foi 200. O resultado final, que apresentou o menor valor da função objetivo, com quatro grupos (círculos, diamantes, triângulos e quadrados) está ilustrado na Figura 4.21.

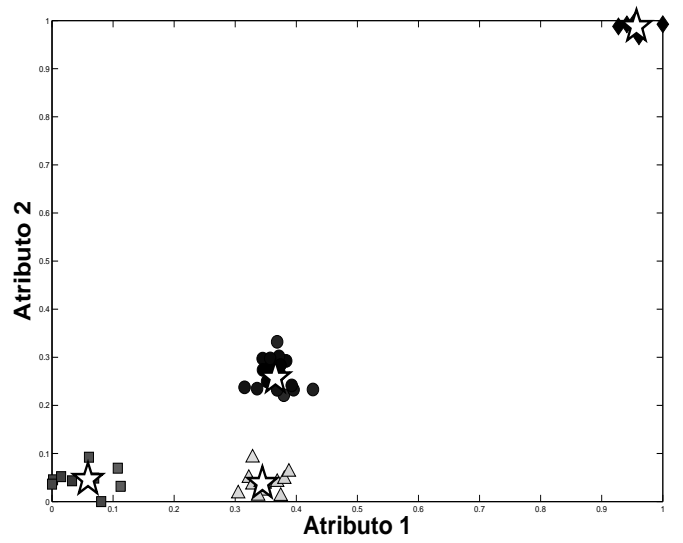

Figura 4.21: Resultado obtido ao aplicar o processo de consenso em cada subespaço da base de dados da Figura 4.17. Quatro grupos foram encontrado (diamantes, círculos, triângulos e quadrados) - centros são representados por "estrelas".

Note que o procedimento encontrou os mesmos quatro grupos que são visualmente esperados. Os resultados individuais em cada subespaço não mostram os quatro grupos encontrados, como discutido anteriormente. Entretanto, esses resultados apresentam informações importantes sobre os objetos e suas proximidades e podem ser usados para construir uma solução consenso mais desejável.

\subsection{Caso Vertical - II}

Neste experimento, imagens descritas por diferentes descritores de imagens são agrupadas. As imagens foram obtidas de (Geusebroek \& Smeulders, 2005; Systems, 2010). Cinco coleções de imagens foram selecionadas, cada qual contendo 111 imagens de um certo objeto físico, assim produzindo um total de 555 imagens de cinco objetos físicos diferentes. As imagens selecionadas estão catalogadas como 422, 656, 792, 915 e 959 em (Systems, 2010) e um exemplo dessas cinco coleções de imagens está ilustrado nas Figuras $4.22,4.23,4.24,4.25$ e 4.26 .

Quatro métodos disponíveis em (Mathias \& Chatzichristofis, 2008; Metadata, 2010) ${ }^{6}$ que calculam dissimilaridades entre imagens foram usados para construir quatro matrizes relacionais $555 \times 555\left(R_{1}, R_{2}, R_{3}\right.$, e $\left.R_{4}\right)$ com pares de distâncias entre todos os objetos (imagens). Cada matriz relacional descreve os dados em um subespaço diferente. O procedimento OMR-FCMdd foi aplicado usando uma versão relacional (Sledge et al.,

\footnotetext{
${ }^{6}$ ScalableColor, ColorLayout, EdgeHistogram e Auto Color Correlogram
} 


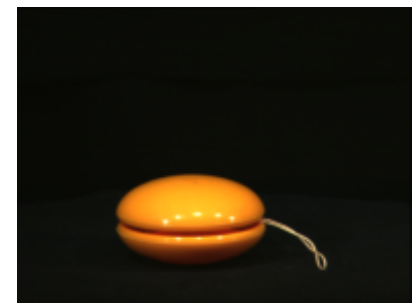

(a)

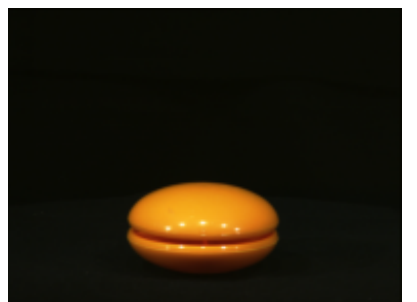

(b)

Figura 4.22: Dois exemplos de imagens 422.

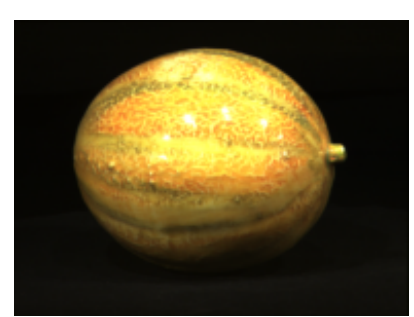

(a)

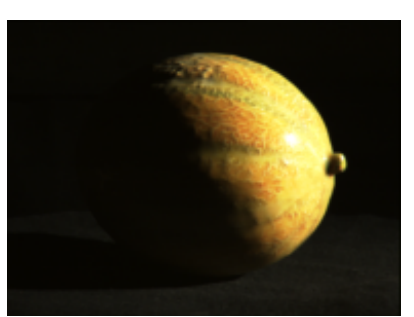

(b)

Figura 4.23: Dois exemplos de imagens 656.

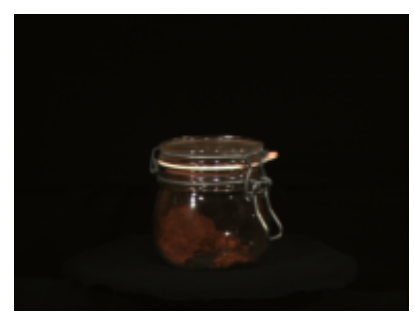

(a)

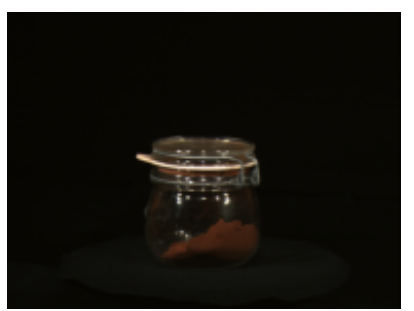

(b)

Figura 4.24: Dois exemplos de imagens 792.

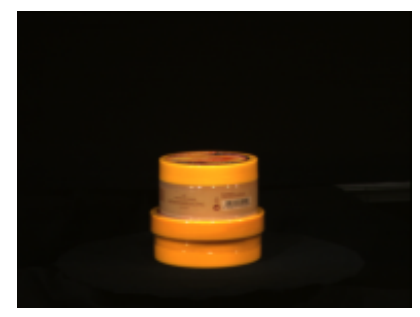

(a)

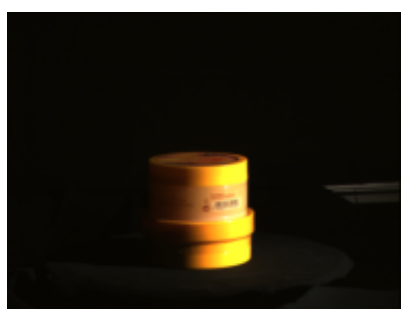

(b)

Figura 4.25: Dois exemplos de imagens 915.

2010) do índice XB discutido na Seção 2.5.2.1 para avaliar as soluções. Tal versão é dita relacional porque pode ser calculada utilizando apenas as distâncias entre objetos disponíveis na matriz de dados relacional, não os objetos em si.

Dado que existem cinco coleções de diferentes imagens, é possível comparar quantitativamente a qualidade dos agrupamentos obtidos com a solução esperada de cinco grupos, e.g., usando os índices externos Jaccard e Rand-Ajustado (Jain \& Dubes, 1988) (Apêndice A). Quanto maior o valor desses índices, mais similar a partição encontrada é da partição 


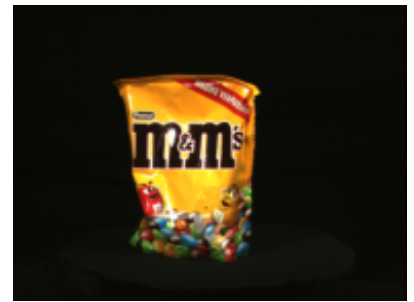

(a)

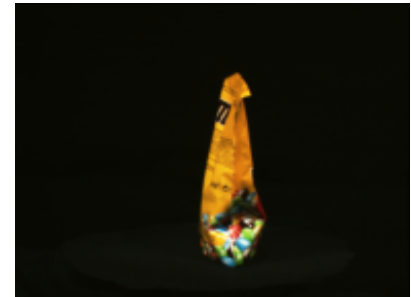

(b)

Figura 4.26: Dois exemplos de imagens 959.

Tabela 4.2: Número de grupos, valor de Jaccard e Rand-Ajustado obtidos pelo procedimento OMR-FCMdd em cada subespaço individualmente, pelo procedimento OMRFCMdd em $R^{+}$e pelo consenso (OMR-ACM-V).

\begin{tabular}{ccccc||cc}
\hline & $\mathbf{R}_{1}$ & $\mathbf{R}_{2}$ & $\mathbf{R}_{3}$ & $\mathbf{R}_{4}$ & $\mathbf{R}^{+}$ & OMR-ACM-V \\
No. de Grupos & 7 & 2 & 2 & 6 & 4 & 6 \\
\hline Jaccard & 0.7788 & 0.2833 & 0.2895 & 0.5182 & 0.5151 & 0.7841 \\
Rand-Ajustado & 0.8483 & 0.1928 & 0.2046 & 0.6066 & 0.5815 & 0.8487 \\
\hline \hline
\end{tabular}

esperada.

O procedimento OMR-FCMdd foi aplicado individualmente em cada subespaço (i.e., matriz relacional) com $k_{\min }=2, k_{\max }=8$ e $M=50$. Além disso, esse procedimento também foi aplicado a uma única matriz $R^{+}=R_{1}+R_{2}+R_{3}+R_{4}$ para produzir um resultado global a partir dos diferentes descritores das imagens (subespaços). Finalmente, o procedimento OMR-ACM-V também foi aplicado para obter uma partição consenso entre os resultados produzidos pelo OMR-FCMdd (aplicado independentemente em cada subespaço, $R_{1}, R_{2}, R_{3}$, e $R_{4}$ ). Os resultados estão ilustrados na Tabela 4.2.

Embora nenhum dos resultados exiba o número esperado de grupos, 5, o procedimento de consenso apresenta uma solução melhor (avaliada pelo Jaccard e Rand-Ajustado) em comparação com o OMR-FCMdd aplicado em cada subespaço individualmente ou na matriz relacional $R^{+}$. A solução consenso é um pouco melhor do que uma das soluções obtidas pelos subespaços individualmente $\left(R_{1}\right)$. Contudo, note que, em aplicações práticas, não existem soluções esperadas para serem usadas no cálculo do Jaccard e Adjusted-Rand, e, portanto, o usuário não sabe se algum dos subespaços (e qual deles) poderia prover uma melhor solução de agrupamento individualmente. 


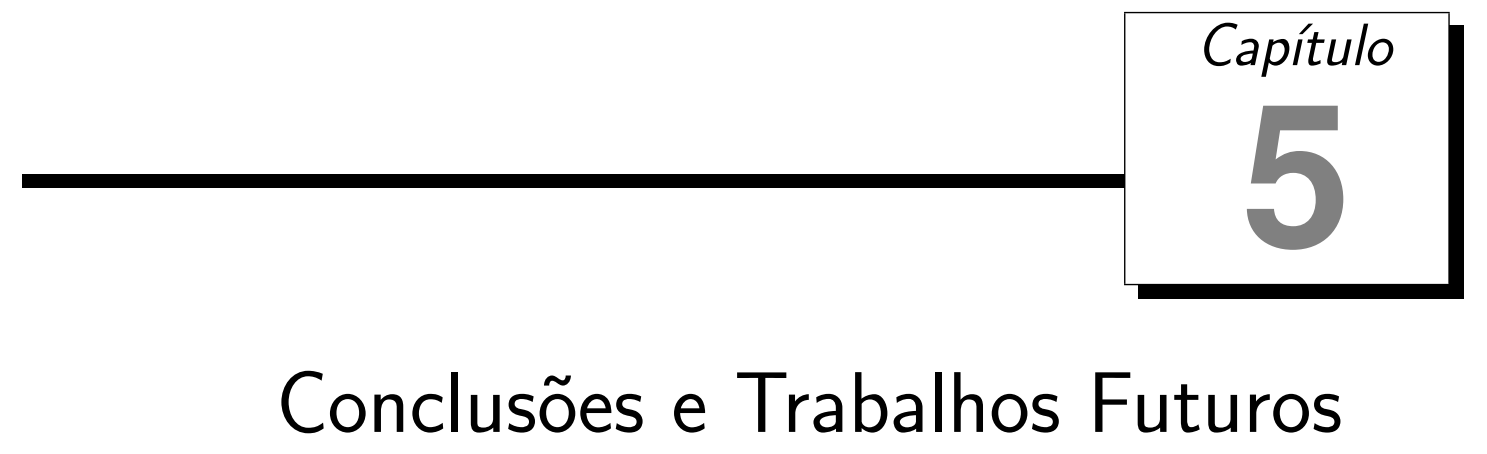

Neste capítulo são apresentadas as conclusões deste trabalho de mestrado e possíveis direções para trabalhos futuros.

\subsection{Conclusões}

Vários algoritmos de agrupamento fuzzy de dados foram propostos na literatura e atualmente são utilizados na prática. Cada algoritmo foi desenvolvido para ser executado em cenários específicos, apresentando vantagens e desvantagens. Neste trabalho de mestrado, tais algoritmos foram descritos e catalogados de acordo com suas características, tais como: formatos de grupos encontrados (esféricos, elipticos, bordas, etc.); matrizes de partição encontradas (fuzzy probabilística ou fuzzy possibilística); dados de entrada ao algoritmo (matriz dos dados, matriz relacional, matriz dos dados com atributos categóricos ou matriz dos dados com valores ausentes); dentre outros. Diversos índices de validação de agrupamento fuzzy foram também investigados e catalogados. Para cada algoritmo de agrupamento e índice estudado foi realizada uma análise de complexidade computacional de tempo de execução e de espaço de memória. Tal análise ajuda na identificação dos algoritmos/índices que demandam menor esforço computacional dependendo das variáveis do problema.

Tais algoritmos e índices foram desenvolvidos originalmente para lidarem apenas com dados centralizados. Contudo, cenários em que os dados estão naturalmente distribuídos em diferentes sítios de dados estão se tornando cada vez mais comuns (cenários distribuídos/paralelos). Baseado nos trabalhos de Kwok et al. (2002); Rahimi et al. (2004) e Modenesi et al. (2007), neste trabalho de mestrado foi desenvolvido um framework para algoritmos de agrupamento em cenários distribuídos. Os algoritmos e índices estudados 
foram generalizados para trabalhar com dados distribuídos utilizando este framework. O framework proposto contempla também o cenário paralelo, em que os dados e o processamento são divididos em diversos processadores com o objetivo de executar os algoritmos mais rapidamente. Uma análise de complexidade de comunicação dos algoritmos e índices distribuídos foi reportada. Com as versões distribuídas dos algoritmos e índices, um método para estimar automaticamente o número de grupos foi apresentado.

Foram estudados também os algoritmos de agrupamento em consenso propostos por Loia et al. (2007) e Pedrycz \& Hirota (2008). Em tais algoritmos, o resultado obtido em cada sítio de dados é combinado para formar uma partição consenso final. Uma das limitações desses algoritmos é que o método de otimização utilizado pode gerar partições ruins, devido ao tratamento inadequado das restrições impostas pelo problema de otimização. Uma segunda limitação é que o número de grupos existente na partição consenso final precisa ser conhecido a priori pelo usuário. Neste trabalho, foi proposto uma melhoria em tais algoritmos para evitar o problema existente no processo de otimização e foi proposto, também, um método para estimar automaticamente o número de grupos ao utilizar esses algoritmos.

Foram apresentados experimentos com a execução de diferentes algoritmos em um cenário paralelo. Tais experimentos demonstraram o ganho de performance obtido pelos algoritmos quando executados em vários processadores em comparação com um único processador. Também foi apresentado experimentos com os aperfeiçoamentos propostos para os algoritmos em consenso, demonstrando melhorias significativas e cenários promissores de aplicação.

\subsection{Contribuições}

As contribuições dos estudos realizados nesta dissertação foram:

- Documentação de vários algoritmos de agrupamento fuzzy de dados e índices de validade fuzzy relativos, apresentando as principais características, vantagens e desvantagens, bem como as análises de complexidade de tempo e espaço.

- Desenvolvimento de um framework para a generalização de vários algoritmos e índices para trabalharem com dados distribuídos e, também, as análises de complexidade de comunicação.

- Aprimoramento do algoritmo de consenso proposto por Loia et al. (2007) e Pedrycz \& Hirota (2008). Foi proposto, também, um método para estimar automaticamente o número de grupos usando tais algoritmos.

- Publicação dos resultados em meios de divulgação internacionais: (Vendramin et al., 2011) e (Coletta et al., 2012). 


\subsection{Publicações Geradas}

Os aperfeiçoamentos propostos nos algoritmos em consenso, bem como o método para estimar automaticamente o número de grupos em tais algoritmos, foram publicados em:

- Vendramin, L., Campello, R. J. G. B., Coletta, L. F. S., and Hruschka, E. R. (2011). Distributed fuzzy clustering with automatic detection of the number of clusters. In International Symposium on Distributed Computing and Artificial Intelligence, volume 91, pag. 133-140. Springer Berlin Heidelberg.

Parte das idéias sobre agrupamento em consenso desenvolvidas na dissertação foram sumarizadas no artigo com co-autoria do aluno:

- Coletta, L. F. S., Vendramin, L., Hruschka, E. R., Campello, R. J. G. B., and Pedrycz, W. (2012). Collaborative fuzzy clustering algorithms: Some refinements and design guidelines. IEEE Transactions on Fuzzy Systems, volume 20, pag. 444-462.

\subsection{Trabalhos Futuros}

A investigação de algoritmos de agrupamento fuzzy relacionais para cenários distribuídos complementaria o trabalho realizado, uma vez que o framework desenvolvido não pode ser diretamente aplicado para este tipo de algoritmo. Adicionalmente, embora a complexidade de comunicação tenha sido reportada neste documento para os algoritmos distribuídos, tal análise é teórica e não abrange problemas físicos de comunicação, tais como latência, largura de banda, perda de pacotes, ruídos, etc. Portanto, um procedimento experimental envolvendo um ambiente distribuído também é um assunto interessante para estudos futuros. 


\section{Referências Bibliográficas}

Agogino, A. \& K. Tumer (2006). Efficient agent-based cluster ensembles. Em Proceedings of the fifth international joint conference on Autonomous agents and multiagent systems, AAMAS '06, New York, NY, USA, pp. 1079-1086. ACM. Citado nas páginas 4 e 92 .

Attiya, H. \& J. Welch (2004). Distributed Computing: Fundamentals, Simulations, and Advanced Topics. McGraw-Hill. Citado nas páginas 3 e 71.

Babuska, R. (1998). Fuzzy Modeling for Control. Norwell, MA, USA: Kluwer Academic Publishers. Citado nas páginas 8, 11, 18, 67, e 108.

Baraldi, A. \& P. Blonda (1999a). A survey of fuzzy clustering algorithms for pattern recognition. i. Systems, Man, and Cybernetics, Part B: Cybernetics, IEEE Transactions on 29(6), 778 - 785. Citado na página 8.

Baraldi, A. \& P. Blonda (1999b). A survey of fuzzy clustering algorithms for pattern recognition. ii. Systems, Man, and Cybernetics, Part B: Cybernetics, IEEE Transactions on 29(6), 786 - 801. Citado na página 8.

Barni, M., V. Cappellini, \& A. Mecocci (1996). Comments on "A possibilistic approach to clustering". IEEE Transactions on Fuzzy Systems 4(3), 393-396. Citado nas páginas 30 e 55 .

Beringer, J. \& E. Hüllermeier (2006). Online clustering of parallel data streams. Data Knowl. Eng. 58(2), 180-204. Citado na página 57.

Bezdek, J. \& J. Dunn (1975). Optimal fuzzy partitions: A heuristic for estimating the parameters in a mixture of normal distributions. Computers, IEEE Transactions on $C$ 24(8), 835 - 838. Citado nas páginas 3 e 15.

Bezdek, J. C. (1973). Cluster validity with fuzzy sets. Cybernetics and Systems 3(3), 58-73. Citado na página 59. 
Bezdek, J. C. (1975). Mathematical models for systematics and taxonomy. Em Proceedings of 8th International Conf. Numerical Taxonomy, pp. 143-166. Citado na página 59.

Bezdek, J. C. (1981). Pattern Recognition with Fuzzy Objective Function Algorithms. Plenum Press. Citado nas páginas 3, 11, 59, e 108.

Bezdek, J. C., C. Coray, R. Gunderson, \& J. Watson (1981a). Detection and characterization of cluster substructure I. linear structure: Fuzzy c-Lines. SIAM Journal on Applied Mathematics 40(2), 339. Citado na página 17.

Bezdek, J. C., C. Coray, R. Gunderson, \& J. Watson (1981b). Detection and characterization of cluster substructure II. fuzzy c-Varieties and convex combinations thereof. SIAM Journal on Applied Mathematics 40(2), 358. Citado na página 18.

Borgelt, C. (2007). Prototype-less fuzzy clustering. Em 2007 IEEE International Fuzzy Systems Conference, pp. 1-6. Citado nas páginas 22 e 23.

Bouguessa, M., S. Wang, \& H. Sun (2006). An objective approach to cluster validation. Pattern Recognition Letters 27, 1419-1430. Citado na página 64.

Bouguessa, M. \& S. R. Wang (2004). A new efficient validity index for fuzzy clustering. Em Proc. 2004 International Conference on Machine Learning and Cybernetics, Volume 3, pp. 26-29. Citado na página 66.

Byrd, R., J. C. Gilbert, \& J. Nocedal (2000). A trust region method based on interior point techniques for nonlinear programming. Math. Progr. 89(1), 149-185. Citado na página 97.

Campello, R. \& E. Hruschka (2006). A fuzzy extension of the silhouette width criterion for cluster analysis. Fuzzy Sets and Systems 157(21), 2858 - 2875. Citado nas páginas 61,64 , e 69.

Cannon, R. L., J. V. Dave, \& J. C. Bezdek (1986). Efficient implementation of the fuzzy c-means clustering algorithms. IEEE Trans. Pattern Anal. Mach. Intell. 8(2), 248-255. Citado na página 55.

Cheng, T. W., D. B. Goldgof, \& L. O. Hall (1998). Fast fuzzy clustering. Fuzzy Sets and Systems 93(1), 49 - 56. Citado na página 49.

Coletta, L. F. S., L. Vendramin, E. R. Hruschka, R. J. G. B. Campello, \& W. Pedrycz (2012). Collaborative fuzzy clustering algorithms: Some refinements and design guidelines. IEEE Transactions on Fuzzy Systems 20(3), 444-462. Citado nas páginas 4, 92, e 124 .

Congiusta, A., D. Talia, \& P. Trunfio (2007). Distributed data mining services leveraging WSRF. Future Generation Computer Systems 23(1), 34-41. Citado nas páginas 3 e 71 . 
Cristea, V., C. Dobre, C. Stratan, F. Pop, \& A. Costan (2010). Large-Scale Distributed Computing and Applications: Models and Trends. Hershey, PA: Information Science Reference - Imprint of: IGI Publishing. Citado nas páginas 3 e 71.

da Silva, J., C. Giannella, R. Bhargava, H. Kargupta, \& M. Klusch (2005). Distributed data mining and agents. Engineering Applications of Artificial Intelligence 18, 791-807. Citado nas páginas 3, 71, e 72 .

da Silva, J. C. \& M. Klusch (2006). Inference in distributed data clustering. Engineering Applications of Artificial Intelligence 19(4), 363 - 369. Citado nas páginas 3, 71, e 72.

da Silva, J. C. \& M. Klusch (2007). Privacy preserving pattern discovery in distributed time series. pp. 207-214. IEEE. Citado nas páginas 3, 71, e 72.

Datta, S., K. Bhaduri, C. Giannella, R. Wolff, \& H. Kargupta (2006). Distributed data mining in Peer-to-Peer networks. IEEE Internet Computing 10, 18-26. Citado nas páginas 3 e 71 .

Datta, S., C. Giannella, \& H. Kargupta (2009). Approximate distributed K-Means clustering over a Peer-to-Peer network. IEEE Transactions on Knowledge and Data Engineering 21, 1372-1388. Citado nas páginas 3 e 74.

Dave, R. N. (1992). Generalized fuzzy c-shells clustering and detection of circular and elliptical boundaries. Pattern Recognition 25(7), 713-721. Citado na página 24.

Dave, R. N. (1996). Validating fuzzy partitions obtained through c-shells clustering. Pattern Recognition Letters 17(6), 613 - 623. Citado na página 60.

Dave, R. N. \& K. Bhaswan (1992). Adaptive fuzzy c-shells clustering and detection of ellipses. IEEE Transactions on Neural Networks 3(5), 643-662. Citado na página 54.

Dhillon, I. S. \& D. S. Modha (2000). A data-clustering algorithm on distributed memory multiprocessors. Em Revised Papers from Large-Scale Parallel Data Mining, Workshop on Large-Scale Parallel KDD Systems, SIGKDD, London, UK, pp. 245-260. SpringerVerlag. Citado nas páginas 3 e 74.

Dimitriadou, E., A. Weingessel, \& K. Hornik (2002). A mixed ensemble approach for the semi-supervised problem. Em Proceedings of the International Conference on Artificial Neural Networks, ICANN '02, London, UK, UK, pp. 571-576. Springer-Verlag. Citado nas páginas 4 e 92 .

Du, Z. \& F. Lin (2005). A novel parallelization approach for hierarchical clustering. Parallel Computing 31, 523-527. Citado nas páginas 3 e 74.

Dunn, J. C. (1973). A fuzzy relative of the isodata process and its use in detecting compact well-separated clusters. Journal of Cybernetics 3, 32-57. Citado nas páginas 3 e 11. 
El-Sonbaty, Y. \& M. A. Ismail (1998). Fuzzy clustering for symbolic data. IEEE Transactions on Fuzzy Systems 6(2), 195 -204. Citado na página 52.

Eschrich, S., J. Ke, L. O. Hall, \& D. B. Goldgof (2003). Fast accurate fuzzy clustering through data reduction. IEEE Transactions on Fuzzy Systems 11(2), 262-270. Citado nas páginas 46 e 47.

Everitt, B. S., S. Landau, \& M. Leese (2001). Cluster Analysis (4tha Edição). Arnold. Citado nas páginas 1 e 58.

Fayyad, U. M., G. Piatetsky-Shapiro, \& P. Smyth (1996). Advances in knowledge discovery and data mining. Capítulo: From data mining to knowledge discovery: an overview, pp. 1-34. Menlo Park, CA, USA: American Association for Artificial Intelligence. Citado na página 1.

Forman, G. \& B. Zhang (2000). Distributed data clustering can be efficient and exact. ACM SIGKDD Explorations Newsletter 2, 34-38. Citado nas páginas 3 e 74.

Freitas, A. \& S. H. Lavington (1997). Mining very large databases with parallel processing. Boston: Kluwer Academic Publishers. Citado na página 71.

Fukuyama, Y. \& M. Sugeno (1989). A new method of choosing the number of clusters for the fuzzy c-means method. Em Proc. 5th Fuzzy Syst. Symp., pp. 247-250. Citado na página 63.

Gan, G., C. Ma, \& J. Wu (2007). Data Clustering: Theory, Algorithms, and Applications. ASA-SIAM. Citado na página 58.

Garg, A., A. Mangla, N. Gupta, \& V. Bhatnagar (2006). PBIRCH: a scalable parallel clustering algorithm for incremental data. pp. 315-316. IEEE. Citado nas páginas 3 e 74 .

Gath, I. \& A. B. Geva (1989). Unsupervised optimal fuzzy clustering. IEEE Trans. Pattern Anal. Mach. Intell. 11(7), 773-780. Citado nas páginas 3, 15, 16, 64, e 65.

Geusebroek, J. M., B. G. J. \& A. W. M. Smeulders (2005). The amsterdam library of object images. Int. J. Comput. Vision 1(61), 103-112. Citado na página 120.

Ghosh, J., A. Strehl, \& S. Merugu (2002). A consensus framework for integrating distributed clusterings under limited knowledge sharing. Em In Proc. NSF Workshop on Next Generation Data Mining, pp. 99-108. Citado nas páginas 4 e 92.

Gustafson, D. \& W. Kessel (1978). Fuzzy clustering with a fuzzy covariance matrix. Em Decision and Control including the 17th Symposium on Adaptive Processes, 1978 IEEE Conference on, Volume 86, pp. 761-766. Citado nas páginas 3 e 14. 
Halkidi, M., Y. Batistakis, \& M. Vazirgiannis (2001). On clustering validation techniques. Journal of Intelligent Information Systems 17, 107-145. Citado na página 64.

Hammouda, K. M. \& M. S. Kamel (2009). Hierarchically distributed Peer-to-Peer document clustering and cluster summarization. IEEE Transactions on Knowledge and Data Engineering 21, 681-698. Citado nas páginas 3 e 74.

Hathaway, R. J. \& J. C. Bezdek (1994). Nerf c-means: Non-euclidean relational fuzzy clustering. Pattern Recognition 27(3), 429 - 437. Citado nas páginas 39 e 40.

Hathaway, R. J. \& J. C. Bezdek (2001). Fuzzy c-means clustering of incomplete data. IEEE Transactions on Systems, Man and Cybernetics, Part B 31(5), 735-744. Citado nas páginas 42, 43, 44, e 45.

Hathaway, R. J., J. W. Davenport, \& J. C. Bezdek (1989). Relational duals of the c-means clustering algorithms. Pattern Recognition 22(2), 205-212. Citado na página 38.

Hoppner, F., F. Klawonn, R. Kruse, \& T. Runker (1999). Fuzzy Cluster Analysis: Methods for Classification, Data Analysis and Image Recognition. John Wiley and Sons. Citado nas páginas $2,7,8,11,15,17,18,24,54,67$, e 108.

Hore, P., L. Hall, \& D. Goldgof (2007a). Single pass fuzzy c means. Em Fuzzy Systems Conference, pp. $1-7$. Citado na página 57.

Hore, P., L. Hall, D. Goldgof, \& W. Cheng (2008). Online fuzzy c means. Em NAFIPS 2008 - 2008 Annual Meeting of the North American Fuzzy Information Processing Society, pp. 1-5. Citado na página 57.

Hore, P., L. O. Hall, \& D. B. Goldgof (2007b). A fuzzy c means variant for clustering evolving data streams. Em 2007 IEEE International Conference on Systems, Man and Cybernetics, Montreal, QC, Canada, pp. 360-365. Citado na página 57.

Hruschka, E. R., R. J. G. B. Campello, \& L. N. de Castro (2006). Evolving clusters in gene-expression data. Information Sciences 176, 1898-1927. Citado na página 61.

Hung, M.-C. \& D.-L. Yang (2001). An efficient fuzzy c-means clustering algorithm. Em ICDM '01: Proceedings of the 2001 IEEE International Conference on Data Mining, Washington, DC, USA, pp. 225-232. IEEE Computer Society. Citado na página 56.

Jain, A. K. (2010). Data clustering: 50 years beyond k-means. Pattern Recognition Letters 31(8), 651 - 666. Citado na página 11.

Jain, A. K. \& R. C. Dubes (1988). Algorithms for Clustering Data. Prentice Hall. Citado nas páginas $7,11,27,121$, e 137.

Jain, A. K., M. N. Murty, \& P. J. Flynn (1999). Data clustering: A review. ACM Comp. Surv. 31, 264-323. Citado na página 58. 
Johnson, E. \& H. Kargupta (2000). Collective, hierarchical clustering from distributed, heterogeneous data. Em M. Zaki \& C.-T. Ho (Eds.), Large-Scale Parallel Data Mining, Volume 1759 of Lecture Notes in Computer Science, pp. 803-803. Springer Berlin / Heidelberg. Citado nas páginas 3 e 71.

Kamel, M. S. \& S. Z. Selim (1994). New algorithms for solving the fuzzy clustering problem. Pattern Recognition 27(3), 421 - 428. Citado na página 56.

Kargupta, H., W. Huang, K. Sivakumar, \& E. Johnson (2001). Distributed clustering using collective principal component analysis. Knowledge and Information Systems 3, 422-448. Citado nas páginas 3, 71, e 72.

Kaufman, L. \& P. Rousseeuw (1990). Finding Groups in Data. Wiley. Citado na página 1.

Kaymak, U. \& M. Setnes (2002). Fuzzy clustering with volume prototypes and adaptive cluster merging. IEEE Transactions on Fuzzy Systems 10(6), 705-712. Citado nas páginas 20 e 21.

Klusch, M., S. Lodi, \& G. Moro (2003). Distributed clustering based on sampling local density estimates. Em Proceedings of the 18th international joint conference on Artificial intelligence, San Francisco, CA, USA, pp. 485-490. Morgan Kaufmann Publishers Inc. Citado nas páginas 3 e 71.

Kolen, J. F. \& T. Hutcheson (2002). Reducing the time complexity of the fuzzy c-means algorithm. IEEE Transactions on Fuzzy Systems 10(2), 263-267. Citado nas páginas $13,14,16,18,19,21,24,26,32,33,36,37,39,44,45,46,48,51$, e 54.

Krishnapuram, R., A. Joshi, O. Nasraoui, \& L. Yi (2001). Low-complexity fuzzy relational clustering algorithms for web mining. IEEE Transactions on Fuzzy Systems 9, 595-608. Citado na página 35.

Krishnapuram, R., A. Joshi, \& L. Yi (1999). A fuzzy relative of the k-medoids algorithm with application to web document and snippet clustering. Em Fuzzy Systems Conference Proceedings, Volume 3, pp. 1281-1286. Citado nas páginas 35 e 36.

Krishnapuram, R. \& J. Keller (1993). A possibilistic approach to clustering. IEEE Transactions on Fuzzy Systems 1(2), 98 -110. Citado nas páginas 28 e 29.

Krishnapuram, R., O. Nasraoui, \& H. Frigui (1992). The fuzzy c spherical shells algorithm: A new approach. IEEE Transactions on Neural Networks 3(5), 663-671. Citado nas páginas 25 e 26.

Krishnapuram, R. J. \& H. Frigui (1996). A comparison of fuzzy shell-clustering methods for the detection of ellipses. IEEE Transactions on Fuzzy Systems 4(2), 193-199. Citado na página 54. 
Krishnapuram, R. J., H. Frigui, \& O. Nasraoui (1993). The fuzzy c quadric shell clustering algorithm and the detection of second-degree curves. Pattern Recognition Letters 14(7), 545 - 552. Citado na página 54.

Krishnapuram, R. J., H. Frigui, \& N. O. (1992). New fuzzy shell clustering algorithms for boundary detection and pattern recognition. Em Proc. SPIE 1607, Volume 1607, pp. 458-465. Citado na página 54.

Kwok, T., K. Smith, S. Lozano, \& D. Taniar (2002). Parallel fuzzy c-means clustering for large data sets. Em Euro-Par 2002 Parallel Processing, Volume 2400 of Lecture Notes in Computer Science, pp. 27-58. Citado nas páginas 3, 74, 75, e 123.

Kwon, S. (1998). Cluster validity index for fuzzy clustering. Electronics Letters $34(22)$, 2176 -2177. Citado na página 62.

Loia, V., W. Pedrycz, \& S. Senatore (2007). Semantic web content analysis: A study in proximity-based collaborative clustering. IEEE Transactions on Fuzzy Systems 15(6), 1294-1312. Citado nas páginas 4, 93, 99, 101, e 124.

Mathias, L. \& A. Chatzichristofis, S. (2008). Lire: Lucene image retrieval - an extensible java cbir library. Em Proceedings of the 16th ACM International Conference on Multimedia, pp. 1085-1088. Citado na página 120.

McQueen, J. B. (1967). Some methods of classification and analysis of multivariate observations. Proc. 5th Berkeley Symp. on Mathematical Statistics and Probability, 281-297. Citado na página 11.

Merugu, S. \& J. Ghosh (2005). A privacy-sensitive approach to distributed clustering. Pattern Recognition Letters 26(4), 399-410. Citado nas páginas 3, 71, e 72.

Metadata, S. (2010). Lire - an open source java content based image retrieval library http://www.semanticmetadata.net/lire/. Citado na página 120.

Milligan, G. W. \& M. C. Cooper (1981). A monte carlo study of thirty internal criterion measures for cluster analysis. Psychometrika 46(2), 187-199. Citado nas páginas 105 e 106.

Modenesi, M., M. Costa, A. Evsukoff, \& N. Ebecken (2007). Parallel fuzzy c-means cluster analysis. Em M. Dayde, J. Palma, A. Coutinho, E. Pacitti, \& J. Lopes (Eds.), High Performance Computing for Computational Science - VECPAR 2006, Volume 4395 of Lecture Notes in Computer Science, pp. 52-65. Springer Berlin / Heidelberg. Citado nas páginas $3,74,75,88$, e 123.

Nikhil, R., K. Pal, J. C. Bezdek, \& T. A. Runkler (1997). Some issues in system identification using clustering. Volume 4, pp. 2524-2529. IEEE. Citado na página 69. 
Olson, C. (1995). Parallel algorithms for hierarchical clustering. Parallel Computing 21, 1313-1325. Citado nas páginas 3 e 74.

Pakhira, M. K., S. Bandyopadhyay, \& U. Maulik (2004). Validity index for crisp and fuzzy clusters. Pattern Recognition 37, 487-501. Citado na página 67.

Pakhira, M. K., S. Bandyopadhyay, \& U. Maulik (2005). A study of some fuzzy cluster validity indices, genetic clustering and application to pixel classification. Fuzzy Sets and Systems 155(2), 191 - 214. Citado na página 88.

Pal, N., K. Pal, \& J. Bezdek (1997). A mixed c-means clustering model. Em Proceedings of 6th International Fuzzy Systems Conference, Barcelona, Spain, pp. 11-21. Citado nas páginas 30 e 31.

Pal, N., K. Pal, J. Keller, \& J. Bezdek (2005). A possibilistic fuzzy c-means clustering algorithm. IEEE Transactions on Fuzzy Systems 13(4), 517-530. Citado na página 32.

Pal, N. R. \& J. C. Bezdek (1995). On cluster validity for the fuzzy c-means model. IEEE Transactions on Fuzzy Systems 3(3), 370-379. Citado nas páginas 61 e 63.

Park, B., H. Kargupta, E. Johnson, E. Sanseverino, D. Hershberger, \& L. Silvestre (2001). Distributed, collaborative data analysis from heterogeneous sites using a scalable evolutionary technique. Applied Intelligence 16, 19-42. Citado nas páginas 3 e 71.

Park, B.-H. \& H. Kargupta (2002). Distributed Data Mining: Algorithms, Systems, and Applications. Em Data Mining Handbook, pp. 341-358. Citado nas páginas 3 e 71.

Pedrycz, W. (2002). Collaborative fuzzy clustering. Pattern Recogn. Lett. 23(14), 16751686. Citado nas páginas 4 e 92.

Pedrycz, W. (2005). Knowledge-based clustering from data to information granules. Hoboken, NJ :: John Wiley,. Citado na página 72.

Pedrycz, W. (2007). Collaborative and knowledge-based fuzzy clustering. International Journal of Innovative Computing, Information and Control 3, 1-12. Citado nas páginas 4, 72 , e 92 .

Pedrycz, W. \& K. Hirota (2008). A consensus-driven fuzzy clustering. Pattern Recognition Letters 29, 1333-1343. Citado nas páginas 4, 92, 93, 94, 95, e 124.

Pedrycz, W. \& P. Rai (2008). Collaborative clustering with the use of fuzzy c-means and its quantification. Fuzzy Sets Syst. 159(18), 2399-2427. Citado nas páginas 4 e 92.

Rahimi, S., M. Zargham, A. Thakre, \& D. Chhillar (2004). A parallel fuzzy c-mean algorithm for image segmentation. Em Fuzzy Information, 2004. Processing NAFIPS '04. IEEE Annual Meeting of the, Volume 1, pp. 234 - 237 Vol.1. Citado nas páginas $3,74,75$, e 123 . 
Silva, J. A. (2010). Substituição de valores ausentes: uma abordagem baseada em um algoritmo evolutivo para agrupamento de dados. Dissertação de Mestrado. Citado na página 42.

Sledge, I., J. Bezdek, T. Havens, \& J. Keller (2010). Relational generalizations of cluster validity indices. Fuzzy Systems, IEEE Transactions on 18(4), 771 -786. Citado na página 120.

Strehl, A. \& J. Ghosh (2003). Cluster ensembles - a knowledge reuse framework for combining multiple partitions. J. Mach. Learn. Res. 3, 583-617. Citado nas páginas 4 e 92.

Systems, I. S. I. (2010). Amsterdam library of object images (aloi) - http://staff. science.uva.nl/〜aloi/. Citado na página 120.

Tan, P. N., M. Steinbach, \& V. Kumar (2005). Introduction to Data Mining (1ª Edição). Addison Wesley. Citado nas páginas 1, 2, 57, e 58.

Tanenbaum, A. S. (2001). Modern Operating Systems (2nd ${ }^{\mathrm{a}}$ Edição). Upper Saddle River, NJ, USA: Prentice Hall PTR. Citado na página 71.

Tanenbaum, A. S. (2002). Computer Networks (4ª Edição). Prentice Hall PTR. Citado na página 71 .

Tang, Y., F. Sun, \& Z. Sun (2005). Improved validation index for fuzzy clustering. Em Proceedings of the American Control Conference, Volume 2, pp. 1120 - 1125. Citado na página 63.

Tian, J., L. Zhu, S. Zhang, \& L. Liu (2005). Improvement and parallelism of k-Means clustering algorithm. Tsinghua Science $\&$ Technology 10, 277-281. Citado nas páginas 3 e 74 .

Timm, H., C. Borgelt, \& a. K. R. Doring, C. (2004). An extension to possibilistic fuzzy cluster analysis. Fuzzy Sets and Systems 147(1), 3-16. Citado nas páginas 30 e 55.

Tsoumakas, G., L. Angelis, \& I. Vlahavas (2004). Clustering classifiers for knowledge discovery from physically distributed databases. Data \& Knowledge Engineering 49, 223-242. Citado nas páginas 3 e 71.

Tumer, K. \& A. Agogino (2008). Ensemble clustering with voting active clusters. Pattern Recognition Letters 29, 1947-1953. Citado nas páginas 4 e 92.

Vendramin, L., R. J. G. B. Campello, L. F. S. Coletta, \& E. R. Hruschka (2011). Distributed fuzzy clustering with automatic detection of the number of clusters. Em International Symposium on Distributed Computing and Artificial Intelligence, Volume 91, pp. 133-140. Springer Berlin / Heidelberg. Citado nas páginas 4, 84, 92, e 124 . 
Vendramin, L., R. J. G. B. Campello, \& E. R. Hruschka (2010). Relative clustering validity criteria: A comparative overview. Statistical Analysis and Data Mining 3(4), 209-235. Citado nas páginas 69, 105, e 106.

Wang, L. \& X. Fu (2005). Data Mining with Computational Intelligence. Springer. Citado na página 2.

Wang, W. \& Y. Zhang (2007). On fuzzy cluster validity indices. Fuzzy Sets and Systems 158(19), 2095 - 2117. Citado nas páginas 61 e 64.

Wu, X., V. Kumar, J. R. Wuinlan, J. Ghosh, K. Yang, H. Motoda, G. J. McLachlan, A. Ng, B. Liu, P. S. Yu, Z. H. Zhou, M. Steinbach, D. J. Hand, \& D. Steinberg (2008). Top 10 algorithms in data mining. Knowledge and Information Systems 14, 1-37. Citado na página 11.

Xie, X. \& G. Beni (1991). A validity measure for fuzzy clustering. IEEE Trans. Pattern Anal. Mach. Intelligence 13, 841-847. Citado na página 61.

$\mathrm{Xu}$, R. \& D. C. Wunsch II (2005). Survey of clustering algorithms. IEEE Trans. Neural Networks 16, 645-678. Citado na página 58.

Xu, R. \& D. C. Wunsch II (2009). Clustering. Wiley / IEEE Press. Citado na página 58.

Yang, M. S. (1993). A survey of fuzzy clustering. Mathl. Comput. Modelling 18(11), 1-16. Citado na página 8.

Yoshinari, Y., W. Pedrycz, \& K. Hirota (1993). Construction of fuzzy models through clustering techniques. Fuzzy Sets and Systems 54(2), 157-165. Citado na página 69.

Zaki, M. J. (1999). Parallel and distributed association mining: a survey. IEEE Concurrency \%, 14-25. Citado na página 72.

Zaki, M. J. (2000). Parallel and distributed data mining: An introduction. Em LargeScale Parallel Data Mining, Volume 1759, pp. 1-23. Berlin, Heidelberg: Springer Berlin Heidelberg. Citado na página 71.

Zaki, M. J. \& Y. Pan (2002). Introduction: Recent developments in parallel and distributed data mining. Distributed and Parallel Databases 11, 123-127. 10.1023/A:1013918601668. Citado nas páginas 3 e 71.

Zhang, S., C. Zhang, \& X. Wu (2004). Knowledge Discovery in Multiple Databases. Springer Verlag. Citado nas páginas 3 e 71. 


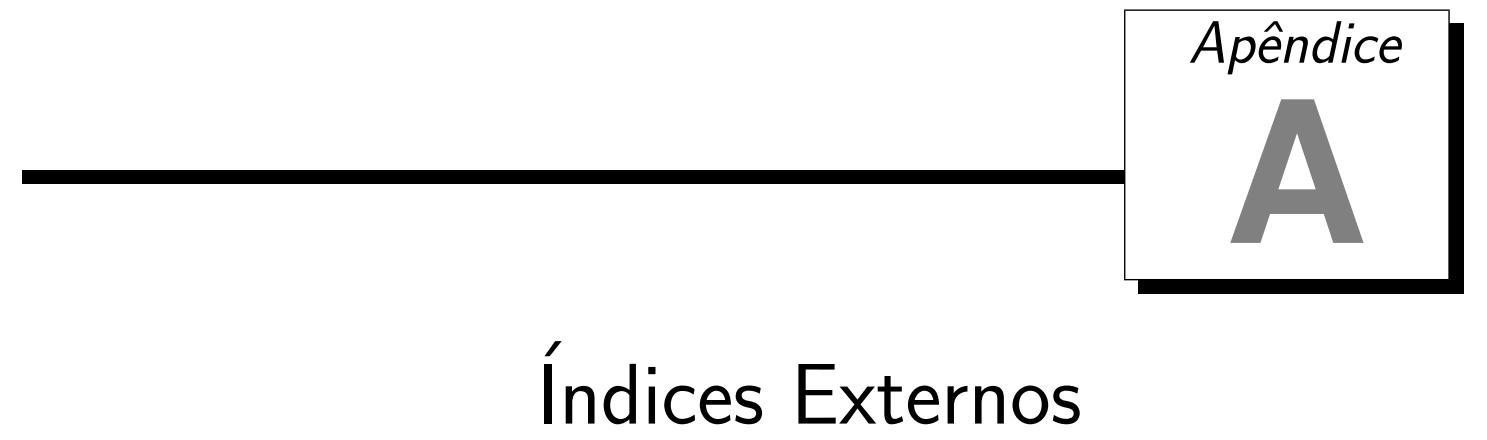

Diferentemente dos índices de validade relativos apresentados na Seção 2.5, os índices de validade externos consistem de medidas absolutas que permitem o uso de bases de dados rotuladas (i.e. os rótulos dos objetos são conhecidos) para a avaliação do desempenho dos resultados obtidos por um algoritmo de agrupamento.

Dois dos índices externos mais conhecidos e utilizados são os índices Jaccard (Jain \& Dubes, 1988) e Rand Ajustado (Jain \& Dubes, 1988). Dadas duas partições $\operatorname{crisp}^{1}$ ( $R$ e $Q)$ da mesma base de dados, em que a partição de referência, $R$, codifica os rótulos das classes, i.e., ela particiona os objetos em $k^{*}$ categorias de objetos conhecidos, enquanto a partição a ser avaliada, $Q$, particiona os dados em $k$ grupos, os índices externos Jaccard e Rand Ajustado são definidos como:

$$
\begin{gathered}
\omega_{\text {Jaccard }}=\frac{a}{a+b+c} \\
\omega_{\text {RandAjustado }}=\frac{a-\frac{(a+c)(a+b)}{M}}{\frac{(a+c)+(a+b)}{2}-\frac{(a+c)(a+b)}{M}}
\end{gathered}
$$

respectivamente, em que $M=a+b+c+d$,

$a$ : $\mathrm{N}^{o}$ de pares de objetos pertencentes à mesma classe em $R$ e ao mesmo grupo em $Q$,

$b$ : $\mathrm{N}^{o}$ de pares de objetos pertencentes à mesma classe em $R$ e a grupos diferentes em $Q$,

$c: \mathrm{N}^{o}$ de pares de objetos pertencentes a classes diferentes em $R$ e ao mesmo grupo em $Q$,

$d: \quad \mathrm{N}^{o}$ de pares de objetos pertencentes a classes diferentes em $R$

\footnotetext{
${ }^{1}$ Para transformar uma partição fuzzy $\mathbf{U} \in \mathbf{M}_{f p}$ em uma partição crisp $\mathbf{U}^{\prime} \in \mathbf{M}_{h}$, basta, para cada objeto $\mathbf{x}_{j}$, fazer com que $u_{i j}=1$ para o grupo $\mathbf{C}_{i}$ com maior valor de pertinência e $u_{i j}=0$ para os demais grupos.
} 
e a grupos diferentes em $Q$.

Os termos $a$ e $d$ são medidas de "concordância", enquanto os termos $b$ e $c$ são medidas de "discordância". Quanto maior o valor do índice externo (valores próximos de 1) maior é a consistência das partições analisadas $(R$ e $Q)$. Quanto menor esse valor, menor é a consistência dessas partições.

Tome como exemplo a Figura A.1, contendo 8 objetos, na qual está ilustrada uma partição de referência, $R$, com dois grupos (círculos e quadrados). Considere que um algoritmo de agrupamento agrupou os 8 objetos ilustrados na Figura A.1 em três grupos (brancos, cinzas e pretos). Deseja-se então mensurar a qualidade da solução obtida pelo algoritmo de agrupamento com três grupos, $Q$, comparando com a partição de referência contendo dois grupos, $R$. Para o cálculo dos índices externos Jaccard e Rand Ajustado, basta calcular os termos $a, b, c$ e $d$. Obtém-se, portanto, $a=5, b=7, c=2, d=14$. Desta forma, o valor obtido pelo índice externo Jaccard é $\omega_{\text {Jaccard }}=\frac{5}{5+7+2}=0,3571$ e o valor obtido pelo índice externo Rand Ajustado é $\omega_{\text {RandAjustado }}=\frac{5-\frac{(5+2)(5+7)}{28}}{\frac{(5+2)+(5+7)}{2}-\frac{(5+2)(5+7)}{28}}=0,3077$. Repare que esses valores indicam que a solução obtida pelo algoritmo de agrupamento não possui alta qualidade, uma vez que os valores obtidos pelos índices externos não estão próximos a 1.
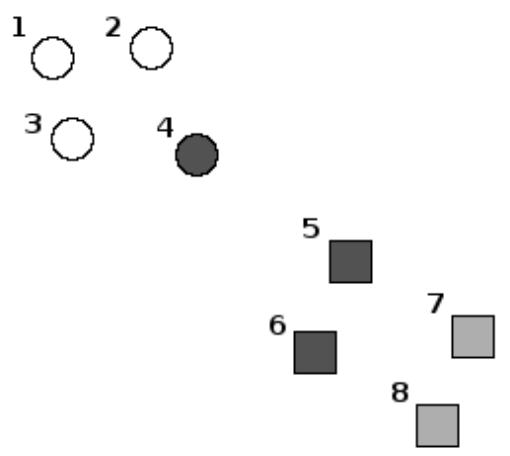

Figura A.1: Exemplo de uma solução contendo 3 grupos (brancos, cinzas e pretos) em comparação com outra solução contendo 2 grupos (círculos e quadrados). 